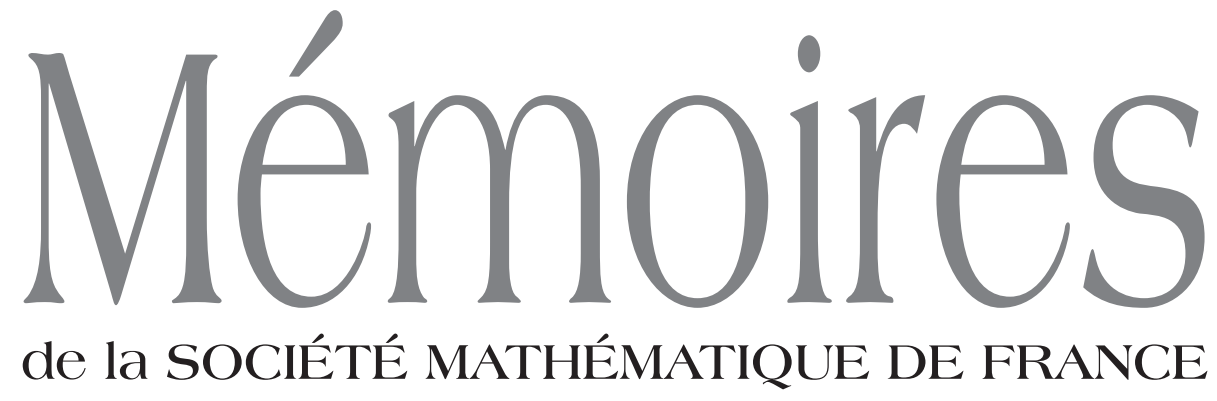

Numéro 118 Nouvelle série
TOPOLOGICAL PROPERTIES OF RAUZY FRACTALS

A. Siegel \& J. M. Thuswaldner

20000 


\section{Comité de rédaction}

$\begin{array}{cc}\text { Jean BARGE } & \text { Daniel HUYBRECHTS } \\ \text { Antoine CHAMBERT-LOIR } & \text { Yves LE JAN } \\ \text { Jean-Marc DELORT } & \text { Wilhem SCHLAG } \\ \text { Julien DUVAL } & \text { Marie-France VIGNÉRAS } \\ \text { Emmanuel GIROUX } & \\ \text { Raphaël KRIKORIAN (dir.) }\end{array}$

Diffusion

Maison de la SMF

AMS

Case 916 - Luminy

P.O. Box 6248

13288 Marseille Cedex 9

Providence RI 02940

France

USA

smf@smf .univ-mrs.fr

WWW . ams . org

\section{Tarifs}

Vente au numéro : $28 €(\$ 42)$

Abonnement Europe : $247 €$, hors Europe : $281 €(\$ 424)$

Des conditions spéciales sont accordées aux membres de la SMF.

\section{Secrétariat : Nathalie Christiaën}

Mémoires de la SMF

Société Mathématique de France

Institut Henri Poincaré, 11, rue Pierre et Marie Curie

75231 Paris Cedex 05, France

Tél : (33) 0144276799 • Fax : (33) 0140469096

revues@smf.ens.fr • http://smf.emath.fr/

(C) Société Mathématique de France 2009

Tous droits réservés (article L 122-4 du Code de la propriété intellectuelle). Toute représentation ou reproduction intégrale ou partielle faite sans le consentement de l'éditeur est illicite. Cette représentation ou reproduction par quelque procédé que ce soit constituerait une contrefaçon sanctionnée par les articles L 335-2 et suivants du CPI.

ISSN 0249-633-X

ISBN 978-2-85629-290-7

Directeur de la publication : Bernard HELFFER 
MÉMOIRES DE LA SMF 118

\title{
TOPOLOGICAL PROPERTIES OF RAUZY FRACTALS
}

\author{
Anne Siegel \\ Jörg M. Thuswaldner
}

Société Mathématique de France 2009

Publié avec le concours du Centre National de la Recherche Scientifique 


\section{A. Siegel}

IRISA, Campus de Beaulieu, 35042 Rennes Cedex, France.

E-mail : Anne.Siegel@irisa.fr

J. M. Thuswaldner

Chair of Mathematics and Statistics, Department of Mathematics and Information Technology, University of Leoben, A-8700 Leoben, Austria.

E-mail : joerg.thuswaldner@unileoben.ac.at

2000 Mathematics Subject Classification. - 28A80, 11A63, 54F65.

Key words and phrases. - Rauzy fractal, tiling, beta-numeration, connectivity, homeomorphy to a disk, fundamental group.

Both authors are supported by the "Amadée" grant FR-13-2008.

The first author is supported by projects ANR-06-JCJC-0073 and BLAN07-1-184548 granted by the French National Research Agency.

The second author is supported by project S9610 granted by the Austrian Science Foundation (FWF). This project is part of the FWF national research network S96 "Analytic combinatorics and probabilistic number theory".

The drawings of the graphs are done with help of the software yFiles. 


\title{
TOPOLOGICAL PROPERTIES OF RAUZY FRACTALS
}

\author{
Anne Siegel, Jörg M. Thuswaldner
}

Abstract. - Substitutions are combinatorial objects (one replaces a letter by a word) which produce sequences by iteration. They occur in many mathematical fields, roughly as soon as a repetitive process appears. In the present monograph we deal with topological and geometric properties of substitutions, in particular, we study properties of the Rauzy fractals associated to substitutions.

To be more precise, let $\sigma$ be a substitution over the finite alphabet $\mathscr{G}$. We assume that the incidence matrix of $\sigma$ is primitive and that its dominant eigenvalue is a unit Pisot number (i.e., an algebraic integer greater than one whose norm is equal to one and all of whose Galois conjugates are of modulus strictly smaller than one). It is well-known that one can attach to $\sigma$ a set $\mathcal{T}$ which is called central tile or Rauzy fractal of $\sigma$. Such a central tile is a compact set that is the closure of its interior and decomposes in a natural way in $n=|\mathscr{G}|$ subtiles $\mathcal{J}(1), \ldots, \mathcal{T}(n)$. The central tile as well as its subtiles are graph directed self-affine sets that often have fractal boundary.

Pisot substitutions and central tiles are of high relevance in several branches of mathematics like tiling theory, spectral theory, Diophantine approximation, the construction of discrete planes and quasicrystals as well as in connection with numeration like generalized continued fractions and radix representations. The questions coming up in all these domains can often be reformulated in terms of questions related to the topology and the geometry of the underlying central tile.

After a thorough survey of important properties of unit Pisot substitutions and their associated Rauzy fractals the present monograph is devoted to the investigation of a variety of topological properties of $\mathcal{T}$ and its subtiles. Our approach is an algorithmic one. In particular, we dwell upon the question whether $\mathcal{T}$ and its subtiles induce a tiling, calculate the Hausdorff dimension of their boundary, give criteria for their connectivity and homeomorphy to a closed disk and derive properties of their fundamental group.

The basic tools for our criteria are several classes of graphs built from the description of the tiles $\mathcal{T}(i)(1 \leq i \leq n)$ as the solution of a graph directed iterated function system and from the structure of the tilings induced by these tiles. These graphs are of interest in their own right. For instance, they can be used to construct the 
boundaries $\partial \mathcal{T}$ as well as $\partial \mathcal{T}(i)(1 \leq i \leq n)$ and all points where two, three or four different tiles of the induced tilings meet.

When working with central tiles in one of the above mentioned contexts it is often useful to know such intersection properties of tiles. In this sense the present monograph also aims at providing tools for "everyday's life" when dealing with topological and geometric properties of substitutions.

Many examples are given throughout the text in order to illustrate our results. Moreover, we give perspectives for further directions of research related to the topics discussed in this monograph.

Résumé (Propriétés topologiques des fractals de Rauzy). - Les fractals de Rauzy apparaissent dans diverses branches des mathématiques telles que la théorie des nombres, les systèmes dynamiques, la combinatoire et la théorie des quasi-cristaux. De nombreuses questions font alors intervenir la structure topologique des fractals. Cette monographie propose une étude systématique des propriétés topologiques des fractals de Rauzy. Les premiers chapitres de ce document rappellent les enjeux mathématiques relatifs aux fractals de Rauzy ainsi que les principaux résultats connus à leur sujet. Sont ensuite discutés des propriétés de pavages, de connexité, d'homéomorphisme à un disque, ainsi que le groupe fondamental de ces ensembles. Les méthodes s'appuient sur des résultats en topologie du plan et sur la construction de graphes pour décrire la structure des pavages associés aux fractals. De nombreux exemples caractéristiques sont présentés. Un chapitre final discute des principales perspectives de recherches liées à cette thématique. 


\section{CONTENTS}

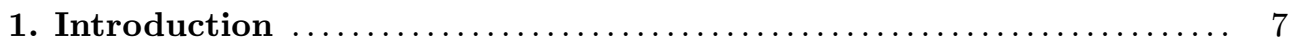

1.1. The role of substitutions in several branches of mathematics $\ldots \ldots \ldots \ldots \quad 7$

1.1.1. Combinatorics ..................................... 7

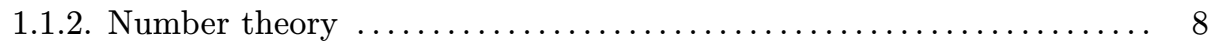

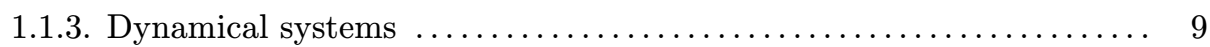

1.1.4. Applications to tiling theory, theoretical physics and discrete

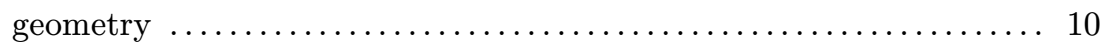

1.2. The geometry of substitutions: Rauzy fractals $\ldots \ldots \ldots \ldots \ldots \ldots \ldots \ldots 12$

1.2.1. The Rauzy fractal for the Tribonacci substitution $\ldots \ldots \ldots \ldots \ldots \ldots 12$

1.2.2. Using a Rauzy fractal and its topological properties ............. 13

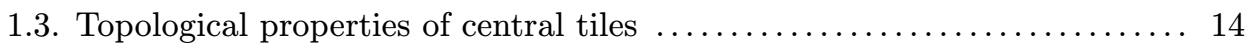

2. Substitutions, central tiles and beta-numeration $\ldots \ldots \ldots \ldots \ldots \ldots$

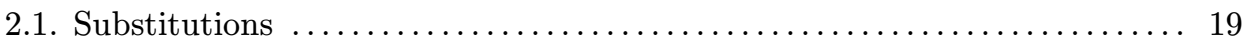

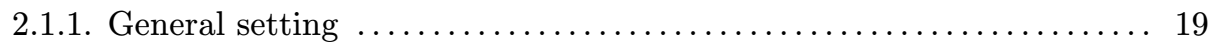

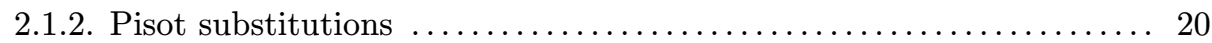

2.2. The central tile associated with a unit Pisot substitution $\ldots \ldots \ldots \ldots \ldots 20$

2.2.1. A broken line associated with a Pisot substitution $\ldots \ldots \ldots \ldots \ldots . \ldots 21$

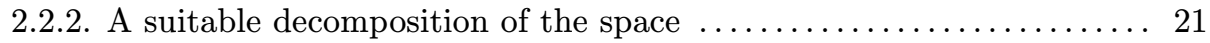

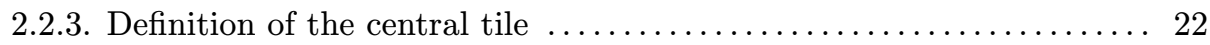

2.3. Central tiles viewed as graph directed iterated function systems $\ldots \ldots \ldots 23$

2.3.1. Disjointness of the subtiles of the central tile ................ 25

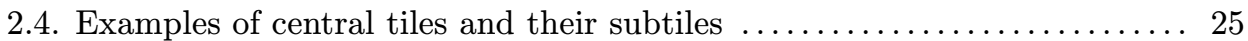

2.5. Recovering beta-numeration from unit Pisot substitutions $\ldots \ldots \ldots \ldots \ldots 28$

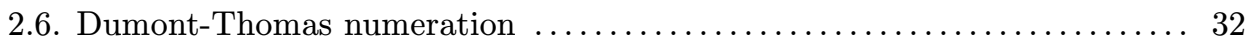

3. Multiple tilings induced by the central tile and its subtiles $\ldots \ldots \ldots 35$

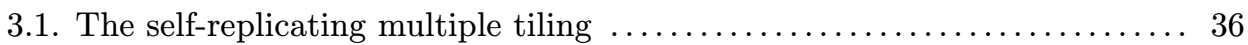

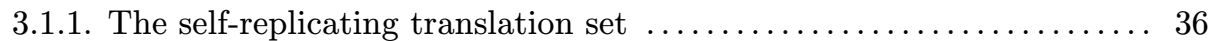

3.1.2. The dual substitution $\mathbf{E}_{1}^{*}$ and the geometric property $(\mathrm{F}) \ldots \ldots \ldots 36$

3.1.3. Definition of the self-replicating multiple tiling $\ldots \ldots \ldots \ldots \ldots \ldots . \ldots 38$

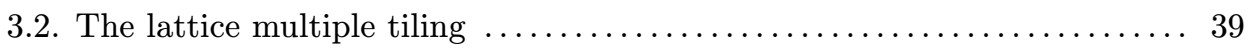

3.3. The tiling property and the Pisot conjecture $\ldots \ldots \ldots \ldots \ldots \ldots \ldots \ldots 43$ 
4. Statement of the main results: topological properties of central tiles 45

4.1. A description of specific subsets of the central tile $\ldots \ldots \ldots \ldots \ldots \ldots \ldots .45$

4.2. Tiling properties of the central tile and its subtiles $\ldots \ldots \ldots \ldots \ldots \ldots \ldots 47$

4.3. Dimension of the boundary of central tiles $\ldots \ldots \ldots \ldots \ldots \ldots \ldots \ldots \ldots . \ldots \ldots$

4.4. Exclusive inner points and the geometric property $(\mathrm{F}) \ldots \ldots \ldots \ldots \ldots$

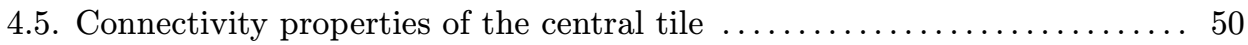

4.6. Disklikeness of the central tile and its subtiles $\ldots \ldots \ldots \ldots \ldots \ldots \ldots \ldots . \ldots 1$

4.7. The fundamental group of the central tile and its subtiles $\ldots \ldots \ldots \ldots .52$

5. Graphs that contain topological information on the central tile ... 53

5.1 . The graph detecting expansions of zero $\ldots \ldots \ldots \ldots \ldots \ldots \ldots \ldots \ldots \ldots \ldots \ldots \ldots$

5.2 . Graphs describing intersections of two subtiles $\ldots \ldots \ldots \ldots \ldots \ldots \ldots \ldots 5$

5.2 .1 . The self-replicating boundary graph $\ldots \ldots \ldots \ldots \ldots \ldots \ldots \ldots \ldots \ldots$

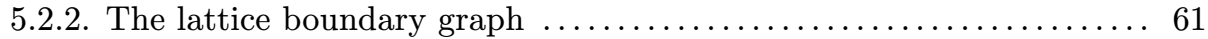

5.3. Graphs related to the connectivity of the central tile $\ldots \ldots \ldots \ldots \ldots \ldots 6$

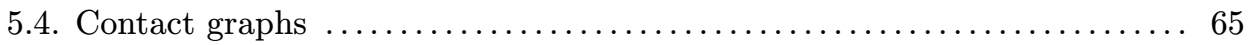

5.5. Triple points and connectivity of the boundary $\ldots \ldots \ldots \ldots \ldots \ldots \ldots \ldots$

5.6. Quadruple points and connectivity of pieces of the boundary $\ldots \ldots \ldots \ldots 73$

5.7. Application of the graphs to the disklikeness criterion $\ldots \ldots \ldots \ldots \ldots \ldots$

\section{Exact statements}

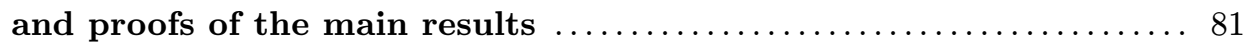

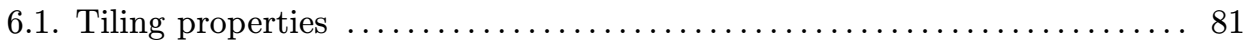

6.2. Dimension of the boundary of the subtiles $\mathcal{J}(i)(i \in \mathscr{C}) \ldots \ldots \ldots \ldots \ldots .84$

6.3. Inner points of $\mathcal{T}$ and the geometric property $(\mathrm{F}) \ldots \ldots \ldots \ldots \ldots \ldots$

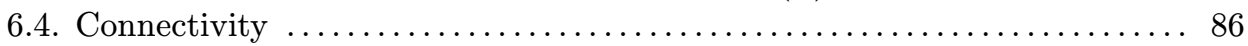

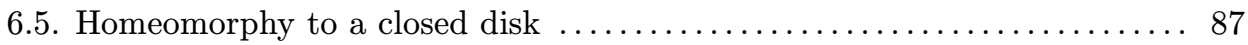

6.5.1. A necessary condition coming from the lattice tiling property . . . 87

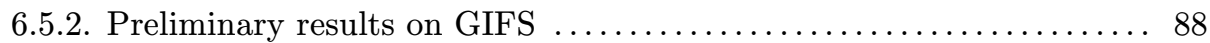

6.5.3. A sufficient condition for the subtiles $\mathcal{T}(i)(i \in \mathscr{C})$ to be disklike . . 91

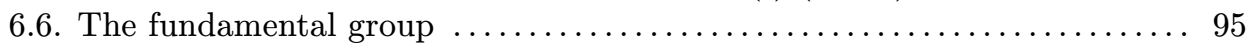

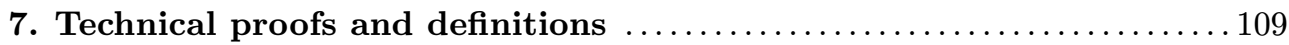

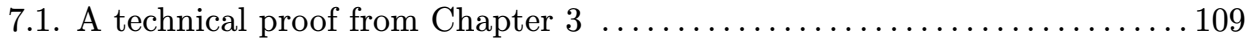

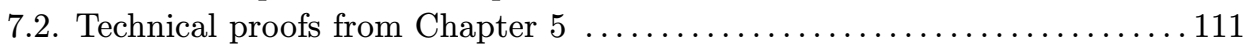

7.3. Details for the quadruple point graph $\ldots \ldots \ldots \ldots \ldots \ldots \ldots \ldots \ldots \ldots \ldots \ldots \ldots \ldots$

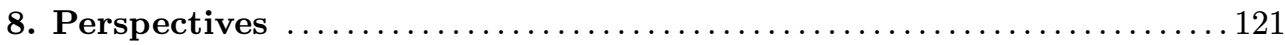

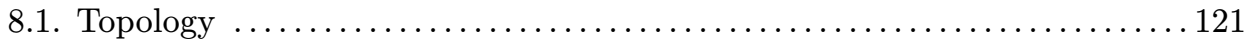

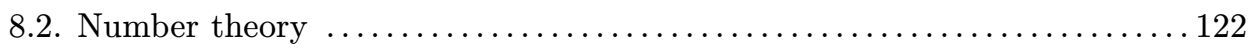

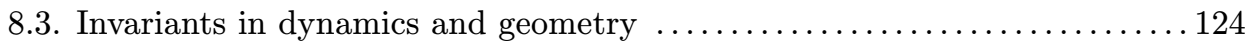

8.4. Effective constructions and generalizations $\ldots \ldots \ldots \ldots \ldots \ldots \ldots \ldots \ldots \ldots \ldots \ldots$

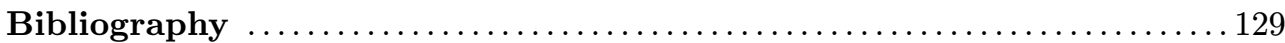




\section{CHAPTER 1}

\section{INTRODUCTION}

The present monograph deals with topological and geometric properties of substitutions. In this introduction we first emphasize on the great importance of substitutions in many fields of mathematics, theoretical physics and computer science. Already in this first part it becomes evident on several places that geometrical objects like Rauzy fractals are intimately related to substitutions and that their topological as well as geometric properties deserve to be studied in order to get information about the underlying substitution. After this general part we give an introductory overview of Rauzy fractals with special emphasis on their topology. We discuss their history and give some details on different ways of their construction. The introduction closes with an outline of the content of this monograph.

\subsection{The role of substitutions in several branches of mathematics}

A substitution (sometimes also called iterated morphism) is a combinatorial object which produces sequences by iteration. It is given by a replacement rule of the letters of a finite alphabet by nonempty, finite words over the same alphabet. Thus substitutions define an iteration process on a finite set in a natural way. Therefore, they can be recovered in many fields of mathematics, theoretical physics and computer science whenever repetitive processes or replacement rules occur.

1.1.1. Combinatorics. - In combinatorics on words, since the beginnings of this domain, substitutions have been used in order to exhibit examples of finite words or infinite sequences with very specific or unusual combinatorial properties. The most famous example is the Thue-Morse sequence defined over the two letter alphabet $\{1,2\}$ as follows. Let the Thue-Morse substitution $\sigma(1)=12, \sigma(2)=21$ be given. This substitution admits two infinite fixed points: the first one begins with all iterations $\sigma^{m}(1)(m \geq 1)$, the second one begins with the words $\sigma^{m}(2)(m \geq 1)$. The first of these fixed points is called Thue-Morse sequence ${ }^{(1)}$. Thue $[\mathbf{1 6 4}, \mathbf{1 6 5}]$ and Morse (see [92]) proved for instance that this infinite sequence is overlap-free, meaning that

(1) Note that the second fixed point emerges from the first one just by exchanging the letters. 
it contains no subword of the shape auaua, where $u$ is a finite word, possibly empty, and $a$ is a single letter (see for instance $[\mathbf{2 1}, \mathbf{4 2}, \mathbf{8 6}, \mathbf{1 1 8}]$ where many other properties of this famous sequence are discussed).

Certain classes of sturmian sequences can be defined in terms of substitutions. Sturmian sequences were introduced in the 1940s as sequences having the smallest complexity among all nonperiodic infinite sequences over a two letter alphabet. In particular, the number of their factors of size $k$ is equal to $k+1$ for each $k \in \mathbb{N}$. A famous characterization relates these sequences to geometry. Indeed, sturmian sequences are exactly the cutting sequences of lines in $\mathbb{R}^{2}$. In particular, first draw grid lines, which are the horizontal and vertical lines through the lattice $\mathbb{Z}^{2}$ in the first quadrant of the plane. Then, traveling along the line $y=\alpha x+\beta$ starting with $x=0$, write down the letter 1 each time a vertical grid line is crossed, and the letter 2 each time a horizontal grid line is crossed [86, Chapter 6]. If $\alpha$ is a quadratic irrational, sturmian sequences can often be related to substitutions $[\mathbf{1 7 0}]$. For instance, if $\beta=0$ and the slope $\alpha \in(0,1)$ of the line is a Sturm number, i.e., a quadratic irrational whose Galois conjugate $\alpha^{\prime}$ satisfies $\alpha^{\prime} \notin(0,1)$, we know that the associated sturmian cutting sequence is the fixed point of a substitution. The most famous case is the fixed point of the Fibonacci substitution $\sigma(1)=12, \sigma(2)=1$ which is associated with $\alpha=\frac{1+\sqrt{5}}{2}$ $[\mathbf{2 0}, \mathbf{7 1}]$. If the slope $\alpha$ is not a quadratic number, then a recoding process is used in order to describe the factors of the sequence by suitable compositions of two "basic" substitutions (see [86, Chapter 6]). The complexity properties of sturmian sequences are used in a variety of applications, such as compression to recover repetitions in DNA sequences [73] or optimal allocation in networks [89].

1.1.2. Number theory. - Since the time when they first appeared, substitutions have been deeply related to number theory: already Thue observed that the ThueMorse sequence classifies integers with respect to the parity of the sum of their binary digits. The Baum-Sweet sequence describes whether the binary expansion of a positive integer contains at least one odd string of zeros. It is obtained as the projection of a substitution on a 4 letter alphabet $[\mathbf{2 1}, \mathbf{3 8}]$. A bridge between substitutions and number theory is also given by the Cobham Theorem, which states that an infinite sequence $\left(u_{k}\right)_{k \geq 0}$ is a letter-to-letter projection of the fixed point of a substitution of constant length $b$ if and only if the letter $u_{k}$ of the sequence is produced by feeding a finite automaton with the expansion of $k$ in base $b[64]$. This theorem allows to derive deep transcendence properties: for instance, the real numbers with continued fraction expansions given by the Thue-Morse sequence, the Baum-Sweet sequence, or the Rudin-Shapiro sequence (see e.g. $[\mathbf{2 1}, \mathbf{1 4 6}]$ for its definition) are all transcendental, the proof being based on the "substitutive" structure of these sequences [2]. Additionally, irrational numbers whose binary expansion is given by the fixed point of a substitution are all transcendental [1]. In the field of diophantine approximation, substitutions produce transcendental numbers which are very badly approximable by cubic algebraic integers [145]; the description of greedy expansions of reals in noninteger base $[\mathbf{6}, \mathbf{1 6 6}]$ by means of substitutions also results in best approximation 
characterizations (see [94] and [118, Chapter 10]). Representations of positive integers by numeration systems related to substitutions are studied in $[\mathbf{7 4 , 7 5 , 7 6 ]}$ (see Section 2.6 below).

1.1.3. Dynamical systems. - Another independent reason for the introduction of substitutions is related to dynamical systems. Indeed, ten years after Thue, Morse rediscovered the Thue-Morse sequence in the field of dynamical systems. Following Poincaré at the beginning of the 20th century, the study of dynamical systems shifted from the research on analytical solutions of differential equations to the study of all possible trajectories and their relations. The research then focused on exhibiting recurrence properties of orbits, that is, properties ensuring that all points will return close to their initial positions. To perform this task in the context of connected surfaces with constant negative curvature, Morse followed an idea proposed by Hadamard: he studied the orbits qualitatively. In Morse's context this means to code a curve by an infinite sequence over the alphabet $\{1,2\}$. Here 1 and 2 occurs according to the boundary of the surface that the curve meets. With this approach and by using the fixed point of the Thue-Morse substitution, Morse succeeded in proving that there indeed exist uniformly non-closed recurrent geodesics [130]. This result initiated the field of symbolic dynamics, that is, studying dynamical systems by coding their orbits as infinite sequences; to this matter, a complicated dynamics over a quite simple space is replaced by a simple dynamics (the shift mapping) over an intricate but combinatorial space made of infinite sequences.

For dynamical systems for which past and future are disjoint, the symbolic dynamical systems are particularly simple and well understood: they are described by a finite number of forbidden words, and they are called shifts of finite type [114]. A partition that induces a coding from a dynamical system onto such a shift of finite type is called a Markov partition [4]: such a partition gives rise to a semiconjugacy from a biinfinite shift to the dynamical system which is one-to-one almost everywhere. A first example has been implicitly given in the invariant Cantor sets of the diffeomorphisms of the sphere studied by Smale [159]. After that the existence of Markov partitions has been established for several classes of dynamical systems, including hyperbolic automorphisms of $n$-dimensional tori and pseudo-Anosov diffeomorphisms of surfaces [55] (note that the proofs are not constructive). The existence of Markov partitions and their associated semiconjugacies is extremely useful in studying many dynamical properties (especially statistical ones); as an example, they are used to prove that hyperbolic automorphisms of the two-dimensional torus are measure-theoretically isomorphic if and only if they have the same entropy [5].

Explicit Markov partitions, however, are generally known only for hyperbolic automorphisms of the two-dimensional torus [4], and they have rectangular shapes. In higher dimensions, a slightly different behavior appears since several results attest that the contracting boundary of a member of a Markov partition cannot be smooth $[56,62]$. Markov partitions have then been proposed by arithmetical means for irreducible hyperbolic toral automorphisms: using homoclinic points of the dynamics 
$[82,151]$ allows to build constant-to-one factor mappings for the dynamical system; however, switching to semiconjugacy mappings is performed only in examples, and the topological properties of the pieces of the partition are not explicit. Another approach was proposed, based on generalized radix representations with a matrix as base $[\mathbf{1 0 6}, \mathbf{1 1 0}, \mathbf{1 1 1}, \mathbf{1 1 2}, \mathbf{1 3 6}$ ] or referring to two-dimensional iteration processes $[\mathbf{2 9}, \mathbf{9 8}]$. These constructions are explicit and geometrical, based on substitutions. The same problem as before occurs when switching from a factor mapping to a semiconjugacy, but this question is tackled in some cases by using symbolic dynamics and the combinatorics of substitutions as we shall detail soon.

In contrast to shifts of finite type we mention highly ordered self-similar systems with zero entropy, which can be loosely defined as systems where the large-scale recurrence structure is similar to the small-scale recurrence structure, or more precisely, as systems which are topologically conjugate to their first return mapping on a particular subset. Their symbolic dynamical systems are generated by substitutions [86, 139] in a natural way. The codings of recurrent geodesics studied by Morse in [130] belong to this class, as well as the return mapping of the expanding flow onto the contracting manifold for hyperbolic toral automorphisms with a unique expanding direction [36]. For these systems, the natural question is to determine their ergodic properties, among which we mention mixing properties or pure discrete spectrum. A very large literature is dedicated to this task (see [86, Chapters 5 and 7]). It was shown that symbolic dynamical systems generated by substitutions have a variety of interesting properties. A specific case is of great interest: when the incidence matrix of the substitution has a unique expanding direction (i.e., when its dominant eigenvalue is a Pisot number; recall that a Pisot number is an algebraic integer greater than one each of whose Galois conjugates has modulus strictly less than one). In this case explicit combinatorial conditions characterize systems with pure discrete spectrum $[\mathbf{3 6}, \mathbf{1 0 1}]$. We will come back to this property in Chapter 3. These conditions are used to prove that the factor mappings induced by the Markov partitions proposed in $[\mathbf{2 9}, \mathbf{9 8}, \mathbf{1 3 6}]$ are indeed semiconjugacies.

\subsubsection{Applications to tiling theory, theoretical physics and discrete ge-} ometry. - Substitutions also appear in physics in connection with quasicrystals. In 1984, aluminium-manganese crystals with icosahedral symmetry where synthesized. However, as crystals were proved to have rotational symmetries confined to orders 2, 3, 4 and 6, the term quasicrystal was invented to describe these new classes of crystals with forbidden symmetry. The definition of quasicrystals and crystals in general has then undergone several modifications and is still not entirely fixed nowadays $[54,119, \mathbf{1 5 3}]$. Nevertheless, a solid is usually considered as a quasicrystal when it has an essentially discrete diffraction diagram. The mathematical question here was to identify atomic structures (or point sets) with a discrete diffraction diagram.

In this context, substitutions are ubiquitous. Indeed, starting from a substitution, one can build a tiling space by considering tilings of the real line by intervals with specific length. The order of intervals in the tilings of this tiling space is governed 
by the factors of a periodic point of the substitution. Such tiling spaces support a natural topology and a minimal and uniquely ergodic $\mathbb{R}$-translation flow.

There is a natural relation between tilings and models of atoms in crystals: a (one-dimensional) tiling of the line can be mapped to a one-dimensional discrete set of points by placing an atom at the end of each tile. The question whether such a configuration is a quasicrystal has been studied since the 1990s: Lee, Moody and Solomyak [113] proved that if the substitution is Pisot (i.e., its incidence matrix has a unique expanding direction), then its diffraction spectrum is purely discrete if and only if the dynamical spectrum of the translation flow on the substitution tiling space is purely discrete from a topological point of view. Recalling that the translation flow on a tiling space is related (even if not exactly equal) to the spectrum of substitutive dynamical systems as mentioned in the previous subsection [36], we realize that a strong bridge exists between theoretical physics and spectral theory of substitutive systems. Criterions for pure discrete spectrum provided in $[\mathbf{3 6}, \mathbf{1 0 1}]$ and already used for Markov partitions can be directly applied in the context of quasicrystals.

One-dimensional tiling flows also appeared in the classification of dynamical systems since every orientable hyperbolic one-dimensional attractor is proved to be either a one-dimensional substitution tiling space or a classical solenoid $[\mathbf{2 2}, \mathbf{3 6}, \mathbf{1 6 9}]$. The consequences of this result will be discussed in Chapter 8 .

From a more combinatorial point of view, a quasicrystal is given by an aperiodic but repetitive structure that plays the role of the lattice in the theory of crystalline structures. Mathematically, we then speak of Meyer sets which are obtained by employing the so-called cut-and-project scheme [129]. In the one-dimensional case, a well-studied family of Meyer sets is given by analogs of the integers in radix representations with respect to a non-integral base, in relation with sturmian sequences [90]. In higher-dimensional cases, however, the situation becomes much more difficult. The well-known Penrose tiling is a quasicrystal since it has essentially discrete diffraction diagram, but defining a wide class of examples of quasicrystals is an open question $[22,105,106,144]$. By analogy with the one-dimensional case, good candidates for cut-and-project schemes (hence, quasicrystals) are given by discrete approximations of planes that are orthogonal to Pisot directions $[\mathbf{2 5 , 3 9 ] . ~ S t i l l ~ b y ~ a n a l o g y ~ w i t h ~ s t u r m i a n ~}$ sequences, such approximations of planes can be generated from one-dimensional substitutions by applying suitable continued fractions algorithms $[\mathbf{2 5}, \mathbf{9 9}]$. However, the large literature dedicated to ergodic properties of multi-dimensional substitutive tiling flows [161] applies with difficulty since the definition of substitutive planar tilings is still not mature [137] and much work remains to be done in this direction.

As a final topic, let us mention that the use of substitutions to describe discrete planes in $\mathbb{R}^{3}$ has recently proved to be very useful in discrete geometry to decide algorithmically whether a discrete patch is a part of a given discrete plane $[\mathbf{2 6}, \mathbf{4 4}, \mathbf{8 5}]$. We will discuss this question in Chapter 8. 


\subsection{The geometry of substitutions: Rauzy fractals}

In the world of substitutions, geometrical objects appeared in 1982 in the work of Rauzy [141]. The motivation of Rauzy was to build a domain exchange in $\mathbb{R}^{2}$ that generalizes the theory of interval exchange transformations $[\mathbf{1 0 4}, \mathbf{1 6 8}]$. Thurston [166] introduced the same geometrical object in the context of numeration systems in noninteger bases. As we shall see, meanwhile it is used in many other contexts.

1.2.1. The Rauzy fractal for the Tribonacci substitution. - To each substitution one can associate an incidence matrix $\mathbf{M}=\left(m_{i j}\right)$ in a natural way. Indeed, $m_{i j}$ counts the occurrences of the letter $i$ in $\sigma(j)$. To build a Rauzy fractal (also called central tile) we restrict to the case of a unit Pisot substitution, i.e., a substitution whose incidence matrix is primitive and has a Pisot unit as dominant eigenvalue. There are mainly two methods of construction for Rauzy fractals.

The first approach is based on formal power series and projections of broken lines to hyperplanes and is inspired by Rauzy's seminal paper [141]. The principle is to consider a periodic point of the substitution, then to represent this sequence as a stair (also called "broken line") in $\mathbb{R}^{n}$, where $n$ denotes the size of the alphabet on which the substitution acts. The next step is to project the vertices of the stair onto the contracting subspace of the incidence matrix, spanned by the eigenvectors corresponding to the Galois conjugates of the dominant eigenvalue of the incidence matrix. Since the projection is performed on a contracting stable space of the matrix, and the object that was projected is a periodic point of the substitution (and, hence, "contracted" by the incidence matrix) the closure of the projection is a compact set. A final step consists in drawing several colors with respect to the direction used in the stair to arrive at each vertex before the projection, and we get the Rauzy fractal.

The standard example is given by the so-called Tribonacci substitution defined as $\sigma(1)=12, \sigma(2)=13, \sigma(3)=1$ which was first studied by Rauzy [141]. The incidence matrix, which counts the number of occurrences of each letter in the images of the letters of the substitution is given by $\left[\begin{array}{lll}1 & 1 & 1 \\ 1 & 0 & 0 \\ 0 & 1 & 0\end{array}\right]$ for this substitution. The dominant eigenvalue satisfies the relation $X^{3}-X^{2}-X-1=0$, justifying the name Tribonacci substitution. The contracting space is two-dimensional. Projecting the "broken line" related to the unique fixed point of the Tribonacci substitution to the contracting plane yields a nice fractal picture, the so-called classical Rauzy fractal $\mathcal{T}$ which is depicted in Figure 1.1 with its subtiles $\mathcal{T}(1)$ (largest subtile), $\mathcal{T}(2)$ (middle size subtile), $\mathcal{T}(3)$ (smallest subtile).

Since this compact set is obtained from the fixed point of the substitution, the selfinduced properties of the fixed point have geometrical consequences: we represent the contracting space as the complex plane $\mathbb{C}$. Denote by $\alpha$ one of the two complex conjugate roots of the polynomial; one has $|\alpha|<1$. With help of $\alpha$, the Rauzy fractal can be written as graph directed iterated function system in the sense of [125] as

$$
\mathcal{T}(1)=\alpha(\mathcal{T}(1) \cup \mathcal{T}(2) \cup \mathcal{T}(3)), \quad \mathcal{J}(2)=\alpha \mathcal{T}(1)+1 \quad \mathcal{T}(3)=\alpha \mathcal{T}(2)+1 .
$$




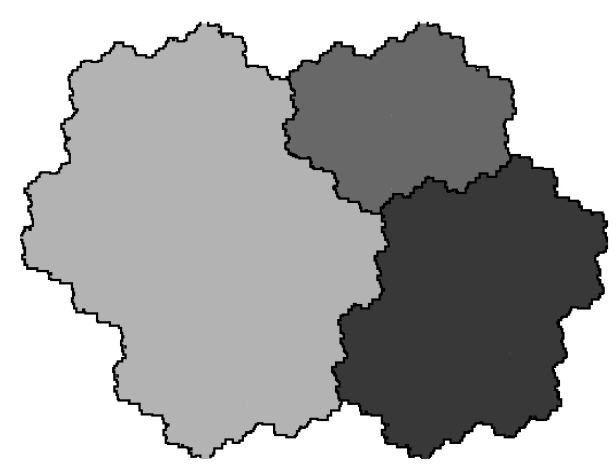

Figure 1.1. The classical Rauzy fractal and its subtiles.

Hence, each subtile is a finite union of translated contracted copies of subtiles. The contraction is given by the Galois conjugate $\alpha$ while the translations depend on the structure of the substitution. The mappings in the graph directed iterated function system are contracting, thus the nonempty compact sets $\mathcal{J}(1), \mathcal{J}(2)$ and $\mathcal{J}(3)$ satisfying (1.1) are uniquely determined [125]; they have nonzero measure and are the closure of their interior [158]. Let us note that the subdivision matrix in the graph directed iterated function system is closely connected to the substitution $\sigma$. In particular, it is the transpose of the incidence matrix of $\sigma$.

The unicity of the solution of such a graph directed iterated function system allows to build the Rauzy fractal in a second way, actually used by Rauzy during his attempts to perform the construction of the fractal (but not published in this way). The principle is to start from a decomposition of a hexagon split into three rhombi. There are two ways for cutting a hexagon into three rhombi: this defines a domain exchange dynamical system on the hexagon. Then, we add pieces to the hexagon and define a new domain exchange so that the first return mapping from the new shape to the hexagon is described by the substitution; then the process is iterated infinitely often. This idea to construct Rauzy fractals is formalized in $[\mathbf{3 0}, \mathbf{1 4 8}]$.

From Rauzy's seminal paper [141], generalizations of the construction have been proposed in different contexts: starting from the investigation of irreducible Pisot units $[\mathbf{3 0}, \mathbf{6 0}, \mathbf{1 2 6}, \mathbf{1 2 7}]$, reducible Pisot units and beta-numeration $[\mathbf{6}, \mathbf{9}, \mathbf{1 6 6}]$, the case of non-unit Pisot numbers $[\mathbf{4 6}, \mathbf{1 5 4}]$ and the hyperbolic case with two expanding directions [29] have been explored so far.

1.2.2. Using a Rauzy fractal and its topological properties. - The large literature dedicated to the Rauzy fractal and its extensions is motivated by the fact that it is used extensively in many domains (as mentioned in Section 1.1). The main reason for the importance of Rauzy fractals is that the iterative procedure to generate infinite words with the help of a substitution is often shifted to a geometric framework and reflects in self-similarity properties that can be studied. Then, the 
main questions to be investigated in each domain can be interpreted as questions related to the topology of the central tile and its tiling properties.

- In number theory, diophantine properties are induced by properties of a distance function to a specific broken line $[\mathbf{9 4}]$ related to the Rauzy fractal and the size of the largest ball contained in it. Finiteness properties of digit representations in numeration systems with non-integer base are related to the fact that $\mathbf{0}$ is an inner point of the Rauzy fractal [19]. Rauzy fractals also characterize purely periodic orbits of representations in numeration systems w.r.t. non-integer base, and yield certain generalizations of Galois' theorem [100].

- Rauzy fractals allow to explicitly build the largest spectral factor induced by a substitutive dynamical system. Explicit Markov partitions for hyperbolic automorphisms of tori are constructed for instance in $[\mathbf{9 8}, \mathbf{1 3 6}]$, actually using Rauzy fractals. Moreover, connectivity properties of Rauzy fractals can be linked to generator properties of the Markov partition [4].

- In tiling theory, Rauzy fractals are used to represent the tiling flow. With help of this representation substitutive systems are proved to be expanding foliations of the space tiling [31].

- In theoretical physics, Rauzy fractals appear as explicit model sets [45].

- In discrete geometry, there are numerous relations between generalized Rauzy fractals and discrete planes as studied for instance in [28]. The shape of pieces generating a discrete plane is tightly related to the shape of Rauzy fractals.

For all these reasons, a thorough study of the topological properties of Rauzy fractals is of great importance. There are several results scattered in literature. For instance, it is known that the Tribonacci Rauzy fractal $\mathcal{T}$ has nice topological behavior ( $\mathbf{0}$ is an inner point of $\mathcal{T}$ and $\mathcal{T}$ is homeomorphic to a closed disk [127]). However, totally different things can appear for other Rauzy fractals: sometimes they are not connected or not simply connected, and $\mathbf{0}$ is not always an inner point of the central tile; see for instance the examples given in [9]. We will review the different contributions to the topological properties of Rauzy fractals that appear in the literature in the next section and in Chapter 4 . However, we have to notice that they are incomplete and often based on examples. Therefore, the main aim of the present monograph is to investigate various topological properties of Rauzy fractals associated to unit Pisot substitutions in a thorough and systematic way.

\subsection{Topological properties of central tiles}

We intend to give an extensive study of the topology of central tiles associated with unit Pisot substitutions. In particular, we emphasize algorithmic criteria for various topological properties.

The monograph starts with two chapters containing a detailed review of substitutions, central tiles and the (multiple) tilings induced by these tiles. These chapters 
are also intended as a survey of basic results related to the geometry of unit Pisot substitutions.

After that, in Chapter 4 we give the statements of the main theorems of the present monograph. Among other things we deal with the following topics:

- We give a criterion that decides whether a given tile induces a tiling. Here we consider both, self-replicating tilings and lattice tilings. Criteria already exist e.g. in terms of super-coincidences $[\mathbf{3 1}, \mathbf{8 1}, \mathbf{1 0 1}]$ as well as in terms of the so-called balanced pair algorithm $[\mathbf{1 1 5}, \mathbf{1 1 6}, \mathbf{1 5 7}]$. A survey of various different tiling criteria is given in [48]. Our criterion has the advantage that it can be applied to lattice tilings even in the reducible case, which was not the case for the other criterions. Moreover, we emphasize that our criterion is an algorithmic necessary and sufficient condition, which is not true for the balanced pair algorithm. This algorithm only terminates if the substitution under consideration admits a tiling.

- We calculate the box-counting dimension of the fractal boundary of the central tile and its subtiles. (For examples as well as certain classes of substitutions such calculations appeared in $[\mathbf{8 4}, \mathbf{9 7}, \mathbf{1 2 7}, \mathbf{1 6 7}]$.) In some cases we are even able to give a formula for the Hausdorff dimension.

- We show that the fact that the origin is an inner point of the central tile is equivalent to a finiteness property, the so-called geometric property $(F)$, of the underlying numeration system (Dumont-Thomas numeration [74]). This was already known in the beta-numeration context $[\mathbf{9}]$. We give a general geometrical proof for this result.

- We give a simple criterion to decide whether the central tile and its subtiles are connected, pursuing the work initiated in $[\mathbf{5 9}, \mathbf{1 4 1}]$.

- We give criteria for the central tile and its subtiles to be homeomorphic to a closed disk. (Examples for disklikeness previously appeared in $[\mathbf{1 2 0}, \mathbf{1 2 7}, \mathbf{1 2 8}]$; in our general approach we use different methods to derive our results.) To this matter we establish a general criterion for a solution of a graph directed iterated function system to be a simple closed curve. This can be applied to the boundary of the subtiles of a central tile. A similar approach as the one we are going to employ has been used in order to prove the homeomorphy to a disk of a class of solutions of iterated function systems associated to numeration systems in the ring of Gaussian integers (see [124]). However, in our situation there is no possibility to conclude from the connectivity of the interior of a tile to its homeomorphy to a disk like it can be done in the case of iterated function systems (see [122]). We have to use several theorems from plane topology to gain our results.

- We give algorithms that can be used to show that the fundamental group of the subtiles of the central tile has certain properties. By doing so, we exhibit examples of central tiles having fundamental groups that are uncountable and not free. 
The underlying idea in all criteria is to match the structure of the graph directed iterated function system that defines the central tile with its tiling properties. All criteria make use and are expressed in terms of graphs.

The graphs we are using to formulate and prove our results are introduced in Chapter 5. Some of them contain the structure of intersections of two or more tiles in the (multiple) tilings induced by the central tile $\mathcal{T}$ and its subtiles $\mathcal{T}(i)(1 \leq i \leq n)$. If the subtiles induce a tiling, they provide a description of $\partial \mathcal{T}$ and $\partial \mathcal{T}(i)(1 \leq i \leq n)$ and even permit to draw these boundaries in an easy way. Other graphs defined in this chapter encode the connectivity of the central tile, its subtiles as well as of certain pieces of their boundary. Summing up, apart from checking the topological properties listed above these graphs are very useful in order to study several properties of $\mathcal{J}$, its subtiles and of the tilings induced by them. This is illustrated by many examples scattered throughout this chapter. In particular, the last section contains a detailed example for the use of the criterion for the homeomorphy of $\mathcal{J}(i)$ to a closed disk.

Chapter 6 contains the proofs of our results. Section 6.5 deserves special attention. It contains the proof of criteria for checking whether $\mathcal{T}$ as well as $\mathcal{T}(i)$ is homeomorphic to a closed disk. In proving these criteria we set up a general theory that admits to decide the disklikeness question for the solution of arbitrary graph directed iterated function systems. The last section contains the exact statement of our results on the fundamental group of $\mathcal{T}$ and $\mathcal{T}(i)$ as well as detailed examples illustrating their application to concrete substitutions. The proofs contained in this chapter make use of general properties of substitutions and central tiles which are reviewed in Chapters 2 and 3, of the graphs defined in Chapter 5 as well as of several results from plane topology.

Chapter 7 contains all the technical proofs we require and gives the details on those graphs which recognize the points in the self-replicating multiple tiling where four different tiles meet.

Chapter 8 contains perspectives for further research. We are confident that the methods contained in this monograph have high potential to be of use in several branches of mathematics. In this chapter we discuss this in more detail and mention the influence of our results to the topology of fractal sets, to number theory (generalized radix representations and continued fractions), as well as to dynamical systems induced by substitutions.

We end this introduction with three tables in which we give an overview of all properties we are dealing with in the present monograph. The first table contains properties which are true for each primitive unit Pisot substitution. In the second table we list properties which are conjecturally true for each primitive unit Pisot substitution. Finally, the third table contains properties that are valid for some but not for all primitive unit Pisot substitutions. In the tables $\mathcal{T}$ denotes the central tile of the respective substitution. Its subtiles are denoted by $\mathcal{J}(i)$. 


\begin{tabular}{|l|l|}
\hline \multicolumn{2}{|c|}{ Properties that are true for each unit Pisot substitution } \\
\hline \hline Property & Comment \\
\hline \hline $\begin{array}{l}\text { The direction of the expanding eigen- } \\
\text { vector of the incidence matrix is irra- } \\
\text { tional. }\end{array}$ & $\begin{array}{l}\text { This follows from the fact that each } \\
\text { Pisot number of degree greater than } \\
\text { one is irrational. }\end{array}$ \\
\hline $\mathcal{T}$ as well as $\mathcal{J}(i)$ is compact. & See Theorem 2.6. \\
\hline $\begin{array}{l}\mathcal{T} \text { as well as } \mathcal{T}(i) \text { is the closure of its } \\
\text { interior. }\end{array}$ & See Theorem 2.6. \\
\hline $\begin{array}{l}\text { The subtiles } \mathcal{T}(i) \text { induce a self- } \\
\text { replicating multiple tiling of the con- } \\
\text { tracting plane. }\end{array}$ & See Proposition 3.7. \\
\hline
\end{tabular}

\begin{tabular}{|l|l|}
\hline \multicolumn{2}{|l|}{ Properties that are conjectured to be true for each unit Pisot substitution } \\
\hline \hline Property & Comment \\
\hline $\begin{array}{l}\text { The strong coincidence condition } \\
\text { holds. }\end{array}$ & $\begin{array}{l}\text { See Definition 2.9. This is true for two } \\
\text { letter substitutions }[\mathbf{3 4}] .\end{array}$ \\
\hline $\begin{array}{l}\text { The subtiles } \mathcal{T}(i) \text { have disjoint interi- } \\
\text { ors. }\end{array}$ & $\begin{array}{l}\text { In view of Theorem 2.10 this is a conse- } \\
\text { quence of the strong coincidence con- } \\
\text { dition. }\end{array}$ \\
\hline $\begin{array}{l}\text { The super-coincidence condition } \\
\text { holds. }\end{array}$ & $\begin{array}{l}\text { This condition was first defined in } \\
{[\mathbf{3 1}, \mathbf{1 0 1}] \text {. We do not give a formal def- }} \\
\text { inition of this condition in this mono- } \\
\text { graph. }\end{array}$ \\
\hline $\begin{array}{l}\text { The subtiles } \mathcal{T}(i) \text { induce a self- } \\
\text { replicating tiling of the contracting } \\
\text { plane. }\end{array}$ & $\begin{array}{l}\text { This is equivalent to the super- } \\
\text { coincidence condition, see Theo- } \\
\text { rem 3.20. }\end{array}$ \\
\hline $\begin{array}{l}\text { If the quotient mapping condition is } \\
\text { true then } \mathcal{J} \text { induces a lattice tiling. }\end{array}$ & $\begin{array}{l}\text { The quotient mapping condition is de- } \\
\text { fined in Definition 3.14. }\end{array}$ \\
\hline
\end{tabular}

\begin{tabular}{|l|l|}
\hline \multicolumn{2}{|c|}{ Properties that are true for some but not for all unit Pisot substitutions } \\
\hline \hline Property & Comment \\
\hline \hline $\begin{array}{l}\text { The quotient mapping condition is } \\
\text { true. }\end{array}$ & See Definition 3.14. \\
\hline The geometric property (F) holds. & $\begin{array}{l}\text { This is a finiteness condition of radix } \\
\text { representations related to substitu- } \\
\text { tions; see Definition 3.6. }\end{array}$ \\
\hline
\end{tabular}




\begin{tabular}{|l|l|}
\hline The origin is an inner point of $\mathcal{J}$. & $\begin{array}{l}\text { This condition is equivalent to the geo- } \\
\text { metric property }(\mathrm{F}) \text {, see Theorems 4.6 } \\
\text { and 4.7. }\end{array}$ \\
\hline Connectivity of $\mathcal{T}$ and $\mathcal{T}(i)$. & $\begin{array}{l}\text { A criterion for connectivity is con- } \\
\text { tained in Theorem } 4.9 .\end{array}$ \\
\hline $\mathcal{T}(i)$ is homeomorphic to a closed disk. & $\begin{array}{l}\text { Criteria for the homeomorphy to a } \\
\text { closed disk are given in Section } 4.6 . \\
\text { See also Section } 5.7 \text { where detailed ex- } \\
\text { amples are given. }\end{array}$ \\
\hline $\begin{array}{l}\text { The fundamental group of } \mathcal{T} \text { is non- } \\
\text { trivial. }\end{array}$ & $\begin{array}{l}\text { See Theorem } 6.23 \text { and the example } \\
\text { given after it. }\end{array}$ \\
\hline $\begin{array}{l}\text { The fundamental group of } \mathcal{T} \text { is un- } \\
\text { countable and not free. }\end{array}$ & $\begin{array}{l}\text { See Theorem } 6.26 \text { and the example } \\
\text { given after it. An informal description } \\
\text { of results on the fundamental group is } \\
\text { given in Section 4.7. }\end{array}$ \\
\hline
\end{tabular}

Acknowledgements: The authors wish to thank P. Arnoux, V. Berthé, A. Hilion, B. Loridant, M. Lustig, and W. Steiner, for many fruitful discussions, especially to exhibit the applications provided in Chapter 8 which contains further perspectives of research on the topic of the present monograph. 


\section{CHAPTER 2}

\section{SUBSTITUTIONS, CENTRAL TILES AND BETA-NUMERATION}

In the present chapter we want to recall the definition and basic properties of the main objects of our study. In the first section we will dwell upon substitutions. Then we will survey basic facts of the central tiles associated to substitutions. Moreover, we shall discuss how these tiles can be represented by a so-called graph directed iterated function system. The chapter closes with a description of the relations between substitutions and beta-numeration and a treatment of Dumont-Thomas numeration, which is a notion of numeration related to substitutions.

\subsection{Substitutions}

2.1.1. General setting. - Let $\mathscr{G}:=\{1, \ldots, n\}$ be a finite set called alphabet whose elements are called letters. The free monoid $\mathscr{C}^{*}$ on the alphabet $\mathscr{G}$ with empty word $\varepsilon$ is defined as the set of finite words on the alphabet $\mathscr{C}$, that is, $\mathscr{C}^{*}:=\{\varepsilon\} \cup \bigcup_{k \in \mathbb{N}} \mathscr{G}^{k}$, endowed with the concatenation mapping. We denote by $\mathscr{Q}^{\mathbb{N}}$ and $\mathscr{C}^{\mathbb{Z}}$ the set of one- and two-sided sequences on $\mathscr{C}$, respectively. The topology of $\mathscr{C}^{\mathbb{N}}$ and $\mathscr{C}^{\mathbb{Z}}$ is the product topology of the discrete topology on each copy of $\mathscr{G}$. Both of these spaces are metrizable.

The length $|w|$ of a word $w \in \mathscr{Q}^{*}$ is defined as the number of letters it contains. For any letter $a \in \mathscr{G}$, we denote by $|w|_{a}$ the number of occurrences of $a$ in $w$. Let $\mathbf{l}$ : $w \in \mathscr{G}^{*} \mapsto\left(|w|_{a}\right)_{a \in \mathscr{G}} \in \mathbb{N}^{n}$ be the natural homomorphism obtained by abelianization of the free monoid, called the abelianization mapping.

A substitution over the alphabet $\mathscr{G}$ is an endomorphism of the free monoid $\mathscr{C}^{*}$ such that the image of each letter of $\mathscr{C}$ is nonempty; to avoid trivial cases (such as projections or permutations of letters), we will always suppose that for at least one letter, say $a$, the length of the successive iterations $\sigma^{k}(a)$ tends to infinity. A substitution naturally extends to the sets of sequences $\mathscr{Q}^{\mathbb{N}}$ and $\mathscr{C}^{\mathbb{Z}}$. We associate to every substitution $\sigma$ its incidence matrix $\mathbf{M}$ which is the $n \times n$ matrix obtained by abelianization, that is, $\mathbf{l}(\sigma(w))=\mathbf{M l}(w)$ for all $w \in \mathscr{Q}^{*}$. 
A one-sided periodic point of the substitution $\sigma$ is a one-sided sequence $u=$ $\left(u_{k}\right)_{k \in \mathbb{N}} \in \mathscr{C}^{\mathbb{N}}$ that satisfies $\sigma^{\nu}(u)=u$ for some $\nu>0$. Moreover, a two-sided periodic point of the substitution $\sigma$ is a two-sided sequence $u=\left(u_{k}\right)_{k \in \mathbb{Z}} \in \mathscr{Q}^{\mathbb{Z}}$ that satisfies $\sigma^{\nu}(u)=u$ for some $\nu>0$ and whose central pair of letters $u_{-1} u_{0}$ belongs to the word $\sigma^{\ell}(a)$ for some $a \in \mathscr{G}$ and some $\ell \in \mathbb{N}$. Each substitution admits one-sided as well as two-sided periodic points (see [139, Proposition V.1]).

2.1.2. Pisot substitutions. - An important property of a substitution is that of primitivity: a substitution $\sigma$ is primitive if there exists an integer $k$ (independent of the letters) such that, for each pair $(a, b) \in \mathscr{Q}^{2}$, the word $\sigma^{k}(a)$ contains at least one occurrence of the letter $b$.

By the definition of primitivity, the incidence matrix $\mathbf{M}$ of a primitive substitution $\sigma$ is a primitive matrix, which implies that it has a simple real positive dominant eigenvalue $\beta$ (here we used the Perron-Frobenius Theorem).

- A substitution $\sigma$ is said to be Pisot if the dominant eigenvalue of its incidence matrix $\mathbf{M}$ is a Pisot number ${ }^{(1)}$.

- A substitution $\sigma$ is said to be unit if the dominant eigenvalue of its incidence matrix $\mathbf{M}$ is a unit.

- A substitution $\sigma$ is said to be irreducible if the algebraic degree of the dominant eigenvalue of its incidence matrix $\mathbf{M}$ is equal to the size of the alphabet.

Note that there exist substitutions whose dominant eigenvalue is a Pisot number but whose incidence matrix has eigenvalues that are not conjugate to the dominant eigenvalue. Examples are $1 \rightarrow 12,2 \rightarrow 3,3 \rightarrow 4,4 \rightarrow 5,5 \rightarrow 1$ and the Thue-Morse substitution $1 \rightarrow 12,2 \rightarrow 21$ (the characteristic polynomial is not irreducible). Such substitutions are not irreducible and they are called reducible.

\subsection{The central tile associated with a unit Pisot substitution}

We now want to give a geometric interpretation of a one-sided periodic point $u \in$ $\mathscr{G}^{\mathbb{N}}$ of a unit Pisot substitution $\sigma$. We first need to introduce some algebraic formalism in order to embed $u$ in a linear subspace spanned by the algebraic conjugates of the dominant eigenvalue of the incidence matrix of $\sigma$; the closure of the "projections" of the prefixes of $u$ will comprise the so-called central tile or Rauzy fractal.

In all what follows, we suppose that $\sigma$ is a primitive unit Pisot substitution over the alphabet $\mathscr{G}$ with $n=|\mathscr{C}|$ letters.

(1) Recall that a Pisot number is an algebraic integer $\beta>1$ such that each Galois conjugate $\beta^{(i)}$ of $\beta$ satisfies $\left|\beta^{(i)}\right|<1$. 
2.2.1. A broken line associated with a Pisot substitution. - Let $u \in \mathscr{G}^{\mathbb{N}}$ be a one-sided periodic point of $\sigma$. The sequence $u$ is embedded as a discrete line in $\mathbb{R}^{n}$ by replacing each letter of $u$ by the corresponding vector in the canonical basis $\left(\mathbf{e}_{1}, \ldots, \mathbf{e}_{n}\right)$ in $\mathbb{R}^{n}$. More precisely, the discrete line has vertices $\left\{\mathbf{l}\left(u_{0} \ldots u_{N-1}\right) ; N \in\right.$ $\mathbb{N}\}$.

2.2.2. A suitable decomposition of the space. - We now need to introduce a suitable decomposition of $\mathbb{R}^{n}$ with respect to eigenspaces of the incidence matrix $\mathbf{M}$ associated to the dominant eigenvalue $\beta$. We denote by $d$ the algebraic degree of $\beta$; one has $d \leq n$, since the characteristic polynomial of $\mathbf{M}$ may be reducible.

Let $r-1$ denote the number of real conjugates of $\beta$; they are denoted by $\beta^{(2)}, \ldots$, $\beta^{(r)}$. Each corresponding eigenspace has dimension one according to the assumption of primitivity. Let $2 s$ denote the number of complex conjugates of $\beta$. They are denoted by $\beta^{(r+1)}, \ldots, \beta^{(r+2 s)}$ with $\beta^{(r+s+k)}=\overline{\beta^{(r+k)}}$ for $k \in\{1, \ldots, s\}$. Each pair of an eigenvector together with its complex conjugate generates a 2-dimensional eigenspace.

Let $\mathbf{v}_{\beta}$ be the dominant eigenvector of ${ }^{t} \mathbf{M}$ normalized in a way that each of its coordinates is an element of $\mathbb{Z}[\beta]$ and let $\mathbf{u}_{\beta}$ be the unique dominant eigenvector of $\mathbf{M}$ satisfying $\left\langle\mathbf{u}_{\beta}, \mathbf{v}_{\beta}\right\rangle=1$. Note that $\mathbf{u}_{\beta}$ has coordinates in $\mathbb{Q}(\beta)$. Moreover, since $\mathbf{u}_{\beta}$ is the dominant eigenvector of a primitive matrix, each of the entries of $\mathbf{u}_{\beta}$ is positive. The same is true for the entries of $\mathbf{v}_{\beta}$. We obtain eigenvectors $\mathbf{u}_{\beta^{(i)}}$ and $\mathbf{v}_{\beta^{(i)}}$ for the Galois conjugates $\beta^{(i)}$ of $\beta$ by replacing $\beta$ by $\beta^{(i)}$ in the coordinates of the vectors. By construction, these vectors satisfy $\left\langle\mathbf{u}_{\beta^{(i)}}, \mathbf{v}_{\beta^{(k)}}\right\rangle=0$ if $i \neq k$ and $\left\langle\mathbf{u}_{\beta^{(i)}}, \mathbf{v}_{\beta^{(k)}}\right\rangle=1$ if $i=k$ (cf. [60, Section 2] for details and note that we identify $\mathbb{R}^{2}$ with $\mathbb{C}$ ).

Using these vectors we introduce a decomposition of $\mathbb{R}^{n}$ as follows.

- The beta-contracting space of the matrix $\mathbf{M}$ is the linear subspace $\mathbb{H}_{c}$ generated by the eigenspaces associated to the Galois conjugates of $\beta$, i.e., by $\mathbf{u}_{\beta^{(2)}}, \ldots$, $\mathbf{u}_{\beta^{(r)}}, \Re\left(\mathbf{u}_{\beta^{(r+1)}}\right), \Im\left(\mathbf{u}_{\beta^{(r+1)}}\right), \ldots, \Re\left(\mathbf{u}_{\beta^{(r+s)}}\right), \Im\left(\mathbf{u}_{\beta^{(r+s)}}\right)$. It has dimension $r+$ $2 s-1=d-1$.

We denote by $\mathbf{h}: \mathbb{H}_{c} \rightarrow \mathbb{H}_{c}$ the restriction of $\mathbf{M}$ to $\mathbb{H}_{c}$. Note that the mapping $\mathbf{h}$ is a contraction whose eigenvalues are the Galois conjugates of $\beta$. We define a suitable norm on $\mathbb{H}_{c}$ by

$$
\forall \mathbf{x} \in \mathbb{H}_{c}, \quad\|\mathbf{x}\|=\max \left\{\left|\left\langle\mathbf{x}, \mathbf{v}_{\beta^{(i)}}\right\rangle\right| ; i=2, \ldots, r+s\right\} .
$$

This implies that

$$
\forall \mathbf{x} \in \mathbb{H}_{c}, \quad\|\mathbf{M} \mathbf{x}\|=\|\mathbf{h} \mathbf{x}\| \leq \max \left\{\left|\beta^{(i)}\right| ; i=2, \ldots, r+s\right\}\|\mathbf{x}\| .
$$

- We denote by $\mathbb{H}_{e}$ the beta-expanding line of $\mathbf{M}$, i.e., the real line generated by the eigenvector $\mathbf{u}_{\beta}$.

- Let $\operatorname{Min}_{\beta}$ be the minimal polynomial of $\beta$. The beta-supplementary space is defined by $\mathbb{H}_{s}=\operatorname{Min}_{\beta}(\mathbf{M}) \mathbb{R}^{n}$ (note that this is a product of the matrix $\operatorname{Min}_{\beta}(\mathbf{M}$ ) by the vectors in $\mathbb{R}^{n}$ ). One checks that $\mathbb{H}_{s}$ is an invariant space of $\mathbf{M}$ that satisfies $\mathbb{R}^{n}=\mathbb{H}_{c} \oplus \mathbb{H}_{e} \oplus \mathbb{H}_{s}$. The space $\mathbb{H}_{s}$ is generated by the eigenspaces 
corresponding to the eigenvalues of $\mathbf{M}$ that are not Galois conjugates to $\beta$. It is trivial if and only if the substitution is irreducible.

From the definition of $\mathbb{H}_{s}$ and the fact that $\mathbf{v}_{\beta}$ belongs to the kernel of $\operatorname{Min}_{\beta}\left({ }^{t} \mathbf{M}\right)$, we deduce that the space $\mathbb{H}_{s}$ is orthogonal to $\mathbf{v}_{\beta}$ (see [81, Lemma 2.5] and [31]), indeed,

$$
\left\langle\mathbf{v}_{\beta}, \mathbb{H}_{s}\right\rangle=\left\langle\mathbf{v}_{\beta}, \operatorname{Min}_{\beta}(\mathbf{M}) \mathbb{R}^{n}\right\rangle=\left\langle\operatorname{Min}_{\beta}\left({ }^{t} \mathbf{M}\right) \mathbf{v}_{\beta}, \mathbb{R}^{n}\right\rangle=0 .
$$

Let $\pi: \mathbb{R}^{n} \rightarrow \mathbb{H}_{c}$ be the projection of $\mathbb{R}^{n}$ onto $\mathbb{H}_{c}$ along $\mathbb{H}_{e} \oplus \mathbb{H}_{s}$, according to the natural decomposition $\mathbb{R}^{n}=\mathbb{H}_{c} \oplus \mathbb{H}_{e} \oplus \mathbb{H}_{s}$. Then, the relation $\mathbf{l}(\sigma(w))=\mathbf{M l}(w)$, which holds for all $w \in \mathscr{C}^{*}$, implies the commutation relation

$$
\forall w \in \mathscr{Q}^{*}, \pi(\mathbf{l}(\sigma(w)))=\mathbf{h} \pi(\mathbf{l}(w)) .
$$

By considering the orthogonality between the vectors $\mathbf{v}_{\beta^{(i)}}$ and the vectors $\mathbf{u}_{\beta^{(k)}}$ for $i \neq k$, we obtain the representation

$$
\forall \mathbf{x} \in \mathbb{R}^{n}, \quad \pi(\mathbf{x})=\sum_{2 \leq i \leq r+2 s}\left\langle\mathbf{x}, \mathbf{v}_{\beta^{(i)}}\right\rangle \mathbf{u}_{\beta^{(i)}}
$$

of $\pi$ in the basis of eigenvectors (again we identified $\mathbb{R}^{2}$ with $\mathbb{C}$ ).

For vectors with rational coordinates, the following relation can be readily obtained by considering Galois conjugates of (2.4).

$$
\forall \mathbf{x}, \mathbf{y} \in \mathbb{Q}^{n}, \quad \pi(\mathbf{x})=\pi(\mathbf{y}) \quad \Longleftrightarrow \quad\left\langle\mathbf{x}, \mathbf{v}_{\beta}\right\rangle=\left\langle\mathbf{y}, \mathbf{v}_{\beta}\right\rangle .
$$

This equivalence means that two points with rational coordinates coincide in the beta-contracting space if and only if they coincide along the beta-expanding line. We will often use this property in the sequel.

2.2.3. Definition of the central tile. - In the irreducible case it is well-known that the Pisot assumption implies that the broken line of $\sigma$ remains at a bounded distance of the expanding direction of the incidence matrix (see [30]). In the reducible case, the discrete line may have other expanding directions, but (2.4) implies that the projection of the discrete line by $\pi$ still provides a bounded set in $\mathbb{H}_{c} \simeq \mathbb{R}^{d-1}$ (for details we refer to $[\mathbf{8 1}$, Section 3.2]).

Definition 2.1 (Central tile / Rauzy fractal). - Let $\sigma$ be a primitive unit Pisot substitution with dominant eigenvalue $\beta$. The central tile (or Rauzy fractal) of $\sigma$ is the projection on the beta-contracting space of the vertices of the discrete line associated to any periodic point $u=\left(u_{k}\right)_{k \in \mathbb{N}} \in \mathscr{C}^{\mathbb{N}}$ of $\sigma$, i.e.,

$$
\mathcal{T}:=\overline{\left\{\pi\left(\mathbf{l}\left(u_{0} \ldots u_{k-1}\right)\right) ; k \in \mathbb{N}\right\}} .
$$

For each $i \in \mathscr{C}$ the subtile $\mathcal{T}(i)$ of the central tile $\mathcal{T}$ is defined depending on the letter associated to the vertex of the discrete line that is projected. More precisely, we set

$$
\mathcal{T}(i):=\overline{\left\{\pi\left(\mathbf{l}\left(u_{0} \ldots u_{k-1}\right)\right) ; k \in \mathbb{N}, u_{k}=i\right\}} \quad(i \in \mathscr{C}) .
$$


REMARK 2.2. - It follows from the primitivity of the substitution $\sigma$ that the definition of $\mathcal{T}$ and $\mathcal{T}(i)(i \in \mathscr{G})$ does not depend on the choice of the periodic point $u \in \mathscr{C}^{\mathbb{N}}$ (see e.g. $\left.[\mathbf{3 0}, \mathbf{6 0}, \mathbf{1 3 9}]\right)$.

By definition, the central tile $\mathcal{T}$ consists of the finite union of its subtiles, i.e.,

$$
\mathcal{J}=\bigcup_{i \in \mathscr{C}} \mathcal{T}(i) .
$$

Examples of central tiles and their subtiles are discussed in Section 2.4.

\subsection{Central tiles viewed as graph directed iterated function systems}

The tiles $\mathcal{T}(i)(i \in \mathscr{G})$ can be viewed as the solution of a so-called graph directed iterated function system, GIFS for short. For the convenience of the reader we recall the definition of GIFS ( $c f$. [125] for a variant of our definition).

Definition 2.3 (GIFS). - Let $G$ be a finite directed graph with set of nodes $\{1, \ldots, q\}$ and set of edges $E$. Denote the set of edges leading from $i$ to $j$ by $E_{i j}$. With each $e \in E$ associate a contracting mapping $\tau_{e}: \mathbb{R}^{n} \rightarrow \mathbb{R}^{n}$. If each node $i$ has at least one outgoing edge we call $\left(G,\left\{\tau_{e}\right\}_{e \in E}\right)$ a GIFS.

The following condition is a separation condition which prevents the solution of a GIFS from having overlaps. Indeed, we will see that under a certain combinatorial condition it is satisfied by the GIFS defining the subtiles $\mathcal{T}(i)(i \in \mathscr{G}$; see Theorems 2.6 and 2.10)

DEFINITION 2.4 (Open set condition). - If there exist nonempty open sets $J_{1}, \ldots, J_{q}$ such that $\left\{\tau_{e}\left(J_{j}\right) ; e \in E_{i j}\right\}$ is a family of pairwise disjoint sets for each fixed $i$ and

$$
J_{i} \supseteq \bigcup_{j=1}^{q} \bigcup_{e \in E_{i j}} \tau_{e}\left(J_{j}\right) \quad(i \in\{1, \ldots, q\})
$$

we say that the GIFS satisfies the generalized open set condition.

It can be shown by a fixed point argument that to a GIFS $\left(G,\left\{\tau_{e}\right\}_{e \in E}\right)$ there corresponds a unique collection of nonempty compact sets $K_{1}, \ldots, K_{q} \subset \mathbb{R}^{n}$ having the property that

$$
K_{i}=\bigcup_{j=1}^{q} \bigcup_{e \in E_{i j}} \tau_{e}\left(K_{j}\right)
$$

The collection $K_{1}, \ldots, K_{q}$ is called GIFS attractor or solution of the GIFS. We will often write (2.6) in the form $K_{i}=\bigcup_{e: i \rightarrow j} \tau_{e}\left(K_{j}\right)$ to emphasize that the union is taken over all edges of $G$ leading away from $i$.

The graph we are going to define now will permit us to view the subtiles $\mathcal{T}(i)$ $(i \in \mathscr{C})$ as solution of a GIFS. The corresponding result is stated immediately after the definition. 
Definition 2.5 (Prefix-suffix graph). - Let $\sigma$ be a substitution over the alphabet $\mathscr{C}$ and let $\mathscr{P}$ be the finite set

$$
\mathscr{P}:=\left\{(p, i, s) \in \mathscr{G}^{*} \times \mathscr{G} \times \mathscr{G}^{*} ; \exists j \in \mathscr{G}, \sigma(j)=p i s\right\} .
$$

Let $\Gamma_{\sigma}$ be the directed graph that is defined in the following way

- The nodes of $\Gamma_{\sigma}$ are the letters of the alphabet $\mathscr{G}$.

- There is a directed edge from $i$ to $j$ labeled by $(p, i, s) \in \mathscr{P}$ if and only if $\sigma(j)=$ pis.

The graph $\Gamma_{\sigma}$ is called prefix-suffix graph of $\sigma$.

An example of a prefix-suffix graph is given in Section 2.4.

TheOrem 2.6. - Let $\sigma$ be a primitive unit Pisot substitution over the alphabet $\mathscr{A}$. The central tile $\mathcal{T}$ as well as each of its subtiles $\mathcal{T}(i)$ is a compact set which is the closure of its interior. Moreover, the subtiles $\mathcal{T}(1), \ldots, \mathcal{J}(n)$ are the solution of the GIFS equation

$$
\forall i \in \mathscr{A}, \mathcal{T}(i)=\bigcup_{\substack{j \in \mathscr{C}, i \stackrel{(p, i, s)}{\longrightarrow} j}} \mathbf{h} \mathcal{T}(j)+\pi \mathbf{l}(p) .
$$

Here the union, which is measure disjoint, is extended over all edges in the prefix-suffix graph of $\sigma$ leading away from the node $i$.

Proof. - In the case where $\sigma$ is irreducible, the fact that $\mathcal{T}$ as well as each $\mathcal{T}(i)$ is compact and equal to the closure if its interior is proved in [158] and the GIFS equation is contained in [45, Theorem 2] (see also [48]). The generalization to the reducible case is given in $[\mathbf{3 1}, \mathbf{8 1}]$. Let $S$ be the shift operator on $\mathscr{G}^{\mathbb{Z}}$ and let $u \in \mathscr{G}^{\mathbb{Z}}$ be a two-sided periodic point of the substitution $\sigma$. Denote by $\bar{\theta}$ the closure of the orbit of $u$ under $S$. The GIFS structure (2.7) comes from the decomposition of every two-sided sequence $w \in \bar{\theta}$. In particular, according to [131], $w=S^{\nu}(\sigma(v))$ with $v \in \bar{\theta}$ and $0 \leq \nu<\left|\sigma\left(v_{0}\right)\right|$, where $v_{0}$ is the 0 -th coordinate of $v$. For more details we also refer to the survey $[\mathbf{4 5}]$.

REMARK 2.7. - Since the mappings $\gamma \mapsto \mathbf{h} \gamma+\pi \mathbf{l}(p)$ are affine, the sets $\mathcal{T}(1), \ldots, \mathcal{T}(n)$ belong to the class of graph directed self-affine sets.

We use the GIFS equation to expand every point of the central tile in terms of powers of $\mathbf{h}$.

Corollary 2.8. - Let $\sigma$ be a primitive unit Pisot substitution over the alphabet $\mathscr{C}$. $A$ point $\delta \in \mathbb{H}_{c}$ belongs to the subtile $\mathcal{T}(i)(i \in \mathscr{C})$ if and only if there exists a walk $\left(p_{k}, i_{k}, s_{k}\right)_{k \geq 0}$ starting at $i=i_{0}$ in the prefix-suffix graph such that

$$
\delta=\sum_{k \geq 0} \mathbf{h}^{k} \pi \mathbf{l}\left(p_{k}\right)
$$


The sequence $\left(p_{k}, i_{k}, s_{k}\right)_{k \geq 0}$ is called an $\mathbf{h}$-ary representation of $\delta$.

In what follows, Greek letters will be used to refer to elements of the contracting space $\mathbb{H}_{c}$.

Proof. - Suppose that $\delta \in \mathcal{T}(i)$. Then the GIFS equation allows to express $\delta$ as $\delta=\mathbf{h} \delta_{1}+\pi \mathbf{l}\left(p_{0}\right)$ where $\delta_{1} \in \mathcal{T}\left(i_{1}\right)$ and $\sigma\left(i_{1}\right)=p_{0} i s_{0}$. By iterating this we get $\delta=\pi \mathbf{l}\left(p_{0}\right)+\cdots+\mathbf{h}^{k} \pi \mathbf{l}\left(p_{k}\right)+\mathbf{h}^{k} \delta_{k}$. Since $\delta_{k}$ and $\pi \mathbf{l}\left(p_{k}\right)$ are uniformly bounded in $k$ and $\mathbf{h}$ is contracting, the power series is convergent and $\delta=\sum_{k>0} \mathbf{h}^{k} \pi \mathbf{l}\left(p_{k}\right)$. The other direction also follows easily.

2.3.1. Disjointness of the subtiles of the central tile. - To ensure that the subtiles are disjoint up to a set of measure zero, we introduce the following combinatorial condition on substitutions.

Definition 2.9 (Strong coincidence condition). - A substitution $\sigma$ over the alphabet $\mathscr{G}$ satisfies the strong coincidence condition if for every pair $\left(j_{1}, j_{2}\right) \in \mathscr{C}^{2}$, there exists $k \in \mathbb{N}$ and $i \in \mathscr{C}$ such that $\sigma^{k}\left(j_{1}\right)=p_{1} i s_{1}$ and $\sigma^{k}\left(j_{2}\right)=p_{2} i s_{2}$ with $\mathbf{l}\left(p_{1}\right)=\mathbf{l}\left(p_{2}\right)$ or $\mathbf{l}\left(s_{1}\right)=\mathbf{l}\left(s_{2}\right)$.

The strong coincidence condition is satisfied by every unit Pisot substitution over a two-letter alphabet [34]. It is conjectured that every Pisot substitution satisfies the strong coincidence condition.

THEOREM 2.10. - Let $\sigma$ be a primitive unit Pisot substitution. If $\sigma$ satisfies the strong coincidence condition, then the subtiles of the central tile have disjoint interiors and the GIFS (2.7) satisfies the generalized open set condition.

Proof. - The proof for the disjointness of the interiors is given in [30] (see also [48]) for the irreducible case and generalized to the reducible case in $[\mathbf{4 5}, \mathbf{8 1}]$. The generalized open set condition is easily seen to be satisfied by the interiors of the sets $\mathcal{T}(i)$ $(i \in \mathscr{G})$.

\subsection{Examples of central tiles and their subtiles}

We now want to give some examples of unit Pisot substitutions and their associated tiles. Moreover, for these examples we will state the topological properties of the central tiles and their subtiles here. All these properties will be proved in the present monograph. Indeed, the examples given here will be used frequently throughout the monograph in order to illustrate our results. 

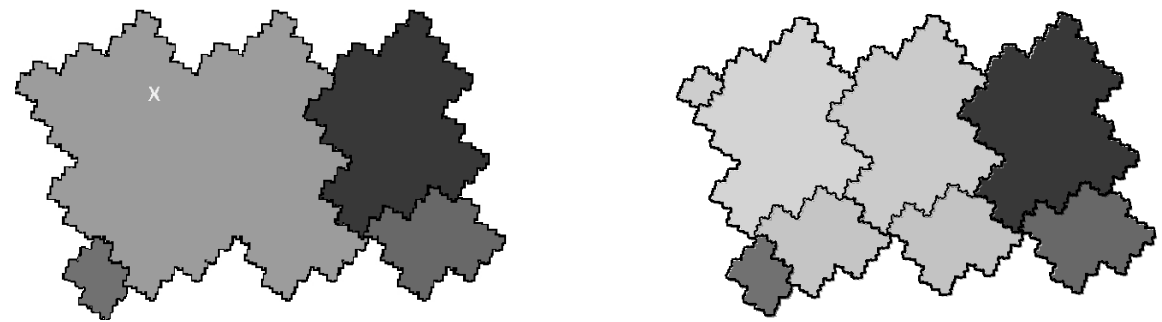

Figure 2.1. The central tile $\mathcal{T}$ of the substitution $\sigma_{0}$ defined in (2.9). On the left hand side the decomposition $\mathcal{T}=\mathcal{T}(1) \cup \mathcal{T}(2) \cup \mathcal{T}(3) \cup \mathcal{T}(4)$ is shown. The white cross " $\mathrm{X}$ " indicates the position of the origin $\mathbf{0}$. On the right hand side the GIFS decomposition of each subtile is illustrated.

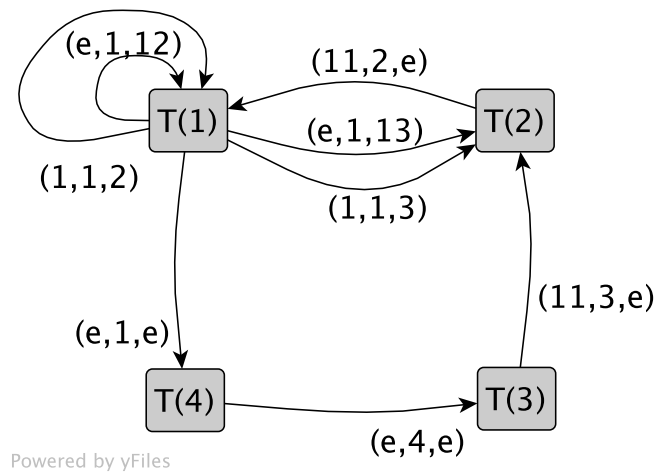

Figure 2.2. The prefix-suffix graph of the substitution $\sigma_{0}$ defined in (2.9). This graph governs the GIFS defining the subtiles $\mathcal{T}(1), \mathcal{J}(2), \mathcal{J}(3), \mathcal{T}(4)$ associated with this substitution. (The letter $e$ denotes the empty word.)

$\sigma_{0}$ : This substitution is our main example. It is defined by

$$
\sigma_{0}(1)=112, \quad \sigma_{0}(2)=113, \quad \sigma_{0}(3)=4, \quad \sigma_{0}(4)=1 .
$$

$\sigma_{0}$ is a reducible primitive unit Pisot substitution whose dominant eigenvalue $\beta$ has degree 3 and satisfies $\beta^{3}-3 \beta^{2}+\beta-1=0$. Its subtiles $\mathcal{T}(1), \mathcal{J}(2), \mathcal{J}(3), \mathcal{T}(4)$ are shown on the left hand side of Figure 2.1. The GIFS decomposition of these subtiles is depicted on the right hand side of this figure. It is goverened by the prefix-suffix graph depicted in Figure 2.2. Equation (2.7) implies that according to this graph the largest subtile $\mathcal{T}(1)$ can be decomposed into five pieces, namely two contracted copies of the largest subtile $\mathcal{T}(1)$, two contracted copies of the second largest subtile $\mathcal{T}(2)$, and one contracted copy of the smallest subtile $\mathcal{T}(4)$. In particular, we obtain the set equation

$$
\mathcal{J}(1)=\mathbf{h} \mathcal{T}(1) \cup\left(\mathbf{h} \mathcal{T}(1)+\pi\left(\mathbf{e}_{1}\right)\right) \cup \mathbf{h} \mathcal{T}(2) \cup\left(\mathbf{h} \mathcal{T}(2)+\pi\left(\mathbf{e}_{1}\right)\right) \cup \mathbf{h} \mathcal{T}(4) .
$$



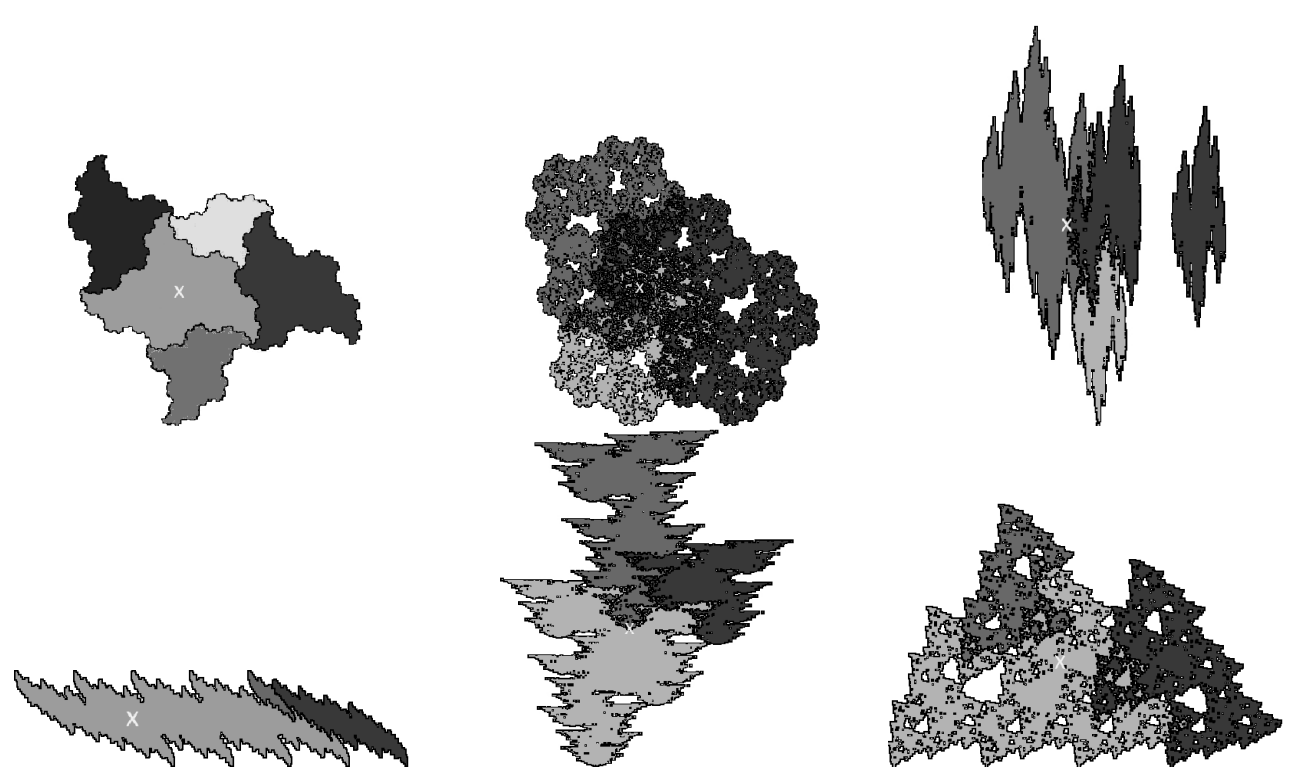

Figure 2.3. In the first line (from left to right) the central tiles of the substitutions $\sigma_{1}, \sigma_{2}$ and $\sigma_{3}$ can be seen. The second line (also from left to right) contains the central tiles of $\sigma_{4}, \sigma_{5}$ and $\sigma_{6}$. In all these central tiles the decomposition in subtiles is indicated by different colors. The white cross " $\mathrm{X}$ " indicates the position of the origin $\mathbf{0}$.

Similar decompositions are obtained for the other subtiles. The second largest subtile $\mathcal{T}(2)$ actually is a contracted copy of the largest subtile $\mathcal{T}(1)$, in particular $\mathcal{T}(2)=\mathbf{h}\left(\mathcal{T}(1)+2 \pi\left(\mathbf{e}_{1}\right)\right)$. The subtile $\mathcal{T}(3)$ is a contracted copy of $\mathcal{T}(2)$, indeed, $\mathscr{T}(3)=\mathbf{h}\left(\mathcal{T}(2)+2 \pi\left(\mathbf{e}_{1}\right)\right)$. Finally, the smallest tile $\mathscr{T}(4)$ is a contracted copy of $\mathcal{T}(3)$, more precisely, we have $\mathcal{T}(4)=\mathbf{h} \mathcal{T}(3)$. Notice that $\mathbf{h}$ includes a rotation of about (but not exactly) $\frac{\pi}{2}$, hence, a contracted subtile $\mathbf{h} \mathcal{J}(j)$ appears with a rotation in the decomposition of $\mathcal{T}(i)$. We will prove that the central tile $\mathcal{T}$ as well as each of its subtiles is homeomorphic to a closed disk. Moreover, $\mathbf{0}$ is an inner point of $\mathcal{T}(1)$.

$\sigma_{1}$ : This substitution is defined by $\sigma_{1}(1)=12, \sigma_{1}(2)=3, \sigma_{1}(3)=4, \sigma_{1}(4)=5$, $\sigma_{1}(5)=1$. It is reducible and its dominant eigenvalue $\beta$ satisfies $\beta^{3}=\beta-1$. The central tile as well as each of its subtiles $\mathcal{T}(1), \ldots, \mathcal{T}(5)$ is homeomorphic to a closed disk which has also been proved in Luo [120] (see Figure 2.3 for a picture of $\mathcal{T}$ and its subtiles). Moreover, $\mathbf{0}$ is an inner point of $\mathcal{T}$.

$\sigma_{2}$ : This substitution is defined by $\sigma_{2}(1)=2, \sigma_{2}(2)=3, \sigma_{2}(3)=12$. It is irreducible and its dominant eigenvalue is equal to the dominant eigenvalue of $\sigma_{1}$. Its central tile $\mathcal{T}$ as well as its subtiles $\mathcal{T}(i)(i=1,2,3)$ are connected and have uncountable fundamental group. $\mathbf{0}$ lies on the boundary of $\mathcal{T}$ (see Figure 2.3). 
$\sigma_{3}$ : This substitution is defined by $\sigma_{3}(1)=3, \sigma_{3}(2)=23, \sigma_{3}(3)=31223$. It is disconnected and $\mathbf{0}$ does not lie in the interior of $\mathcal{T}$ (see Figure 2.3).

$\sigma_{4}$ : This substitution is defined by $\sigma_{4}(1)=11112, \sigma_{4}(2)=11113, \sigma_{4}(3)=1$. It is irreducible and its central tile $\mathcal{J}$ as well as its subtiles have uncountable fundamental group. $\mathbf{0}$ is contained in the interior of $\mathcal{T}$ (see Figure 2.3).

$\sigma_{5}$ : This substitution is defined by $\sigma_{5}(1)=123, \sigma_{5}(2)=1, \sigma_{5}(3)=31$. It is irreducible and its central tile has uncountable fundamental group. Moreover, $\mathbf{0}$ is not an inner point of the central tile (see Figure 2.3).

$\sigma_{6}$ : This substitution is defined by $\sigma_{6}(1)=12, \sigma_{6}(2)=31, \sigma_{6}(3)=1$. It is irreducible and its central tile has uncountable fundamental group (see Figure 2.3).

For the sake of completeness as well as for further reference we provide the following table which gives a summary on properties of the polynomials associated to $\sigma_{0}, \ldots, \sigma_{6}$. In the last column a "=" is printed if all Galois conjugates of the dominant eigenvalue $\beta$ have the same modulus; different moduli of the Galois conjugates are indicated by "f”.

\begin{tabular}{|c|c|c|c|c|}
\hline Subst. & $\begin{array}{c}\text { Characteristic } \\
\text { Polynomial }\end{array}$ & $\begin{array}{c}\text { Minimal polynomial } \\
\text { of the dominant } \\
\text { eigenvalue } \beta\end{array}$ & $\begin{array}{c}\text { Roots } \\
\text { (approximated values) }\end{array}$ & $\begin{array}{l}\text { Moduli } \\
\text { of } \\
\text { conjug. }\end{array}$ \\
\hline$\sigma_{0}$ & $x^{4}-2 x^{3}-2 x^{2}-1$ & $x^{3}-3 x^{2}+x-1$ & $\begin{array}{c}2.769292354 \\
0.1153538228 \pm 0.589742805 i\end{array}$ & $=$ \\
\hline$\sigma_{1}$ & $x^{5}-x^{4}-1$ & $x^{3}-x-1$ & $\begin{array}{c}1.324717957 \\
-0.6623589786 \pm 0.562279512 i\end{array}$ & $=$ \\
\hline$\sigma_{2}$ & $x^{3}-x-1$ & $x^{3}-x-1$ & $\begin{array}{c}1.324717957 \\
-0.6623589786 \pm 0.562279512 i\end{array}$ & $=$ \\
\hline$\sigma_{3}$ & $x^{3}-3 x^{2}-3 x+1$ & $x^{3}-3 x^{2}-3 x+1$ & $\begin{array}{c}3.214319743 \\
-0.6751308705,0.4608111271\end{array}$ & $\neq$ \\
\hline$\sigma_{4}$ & $x^{3}-4 x^{2}-4 x-1$ & $x^{3}-4 x^{2}-4 x-1$ & $\begin{array}{c}4.864536512 \\
-0.4322682562 \pm 0.136797606 i\end{array}$ & $=$ \\
\hline$\sigma_{5}$ & $x^{3}-2 x^{2}-x+1$ & $x^{3}-2 x^{2}-x+1$ & $\begin{array}{c}2.246979603 \\
-0.8019377358,0.554958132\end{array}$ & $\neq$ \\
\hline$\sigma_{6}$ & $x^{3}-x^{2}-x-1$ & $x^{3}-x^{2}-x-1$ & $\begin{array}{c}1.839286755 \\
-0.4196433776 \pm 0.6062907292 i\end{array}$ & $=$ \\
\hline
\end{tabular}

\subsection{Recovering beta-numeration from unit Pisot substitutions}

Let $\beta>1$ be a real number. The (Renyi) $\beta$-expansion of a real number $\xi \in[0,1)$ is defined as the sequence $d_{\beta}(\xi)=\left(a_{i}\right)_{i \geq 1}$ over the alphabet $\mathscr{G}_{\beta}:=\{0,1, \ldots,\lceil\beta\rceil-1\}$ produced by the $\beta$-transformation $T_{\beta}: x \mapsto \beta x(\bmod 1)$. In particular, we set $\forall i \geq 1, a_{i}=\left\lfloor\beta T_{\beta}^{i-1}(x)\right\rfloor$. It is easy to see that we have $\xi=\sum_{i \geq 1} \frac{a_{i}}{\beta^{i}}$ (see $[\mathbf{1 3 5}]$ ). Thus the sequence $d_{\beta}(\xi)$ is a generalization of the classical sequences of decimal and 
binary digits. Note that $d_{\beta}(\xi)$ is also obtained by the "greedy" algorithm which associates $\left(a_{i}\right)_{i \geq 0}$ to $\xi \in[0,1)$ in a way that it is maximal w.r.t. the lexicographic order.

It is well-known that the $\beta$-expansion $\left(\left\lfloor\beta T_{\beta}^{i-1}(1)\right\rfloor\right)_{i \geq 1}$ of 1 plays a crucial role. We denote it by $d_{\beta}(1)=\left(t_{i}\right)_{i \geq 1}$. Let us stress the fact that this infinite expansion cannot be obtained by a greedy algorithm.

- If $d_{\beta}(1)$ is finite with length $n$ (which means that $t_{k}=0$ for $k>n$ ), an infinite expansion of 1 is given by $d_{\beta}^{*}(1)=\left(t_{1} \ldots t_{n-1}\left(t_{n}-1\right)\right)^{\infty}$.

- If $d_{\beta}(1)$ is infinite we define $d_{\beta}^{*}(1)=d_{\beta}(1)$.

The knowledge of this improper expansion $d_{\beta}^{*}(1)$ of 1 allows to decide whether a given word over $\mathscr{C}_{\beta}$ is the $\beta$-expansion of some real number. Indeed, by a classical result of Parry $[53, \mathbf{1 3 5}]$, a finite or infinite word $a_{1} a_{2} \ldots$ is the $\beta$-expansion of a real number in $[0,1)$ if and only if all its suffixes are lexicographically smaller than $d_{\beta}^{*}(1)$. In the case where $\beta$ is a Pisot number, it is known that $d_{\beta}^{*}(1)$ is eventually periodic and every element in $\mathbb{Q}(\beta) \cap[0,1)$ has eventually periodic $\beta$-expansion according to $[49,150]$.

In what follows we assume that $\beta$ is a Pisot unit. Let us denote by

$$
d_{\beta}^{*}(1)=t_{1}^{*} \ldots t_{m}^{*}\left(t_{m+1}^{*} \ldots t_{n}^{*}\right)^{\infty}
$$

the eventually periodic expansion $d_{\beta}^{*}(1)$. Note that this is the defining equation of the numbers $n$ and $m$. Here $m<n$ because $d_{\beta}^{*}(1)$ is defined in a way that there is a period $\left(t_{m+1}^{*} \ldots t_{n}^{*}\right)^{\infty}$ of length at least one.

The $\beta$-transformation induces a decomposition of every positive real number in a $\beta$-fractional and a $\beta$-integral part as follows. For every $x \in \mathbb{R}^{+}$, let $N \in \mathbb{N}$ be the smallest integer satisfying $\beta^{-N} x \in[0,1)$ and $d_{\beta}\left(\beta^{-N} x\right)=a_{-N+1} a_{-N+2} \ldots$ Then

$$
x=\underbrace{a_{-N+1} \beta^{N-1}+\cdots+a_{-1} \beta+a_{0}}_{\beta \text {-integral part }}+\underbrace{\frac{a_{1}}{\beta}+\frac{a_{2}}{\beta^{2}}+\cdots}_{\beta \text {-fractional part }}
$$

We define the set of $\beta$-integers as the set of positive real numbers with no $\beta$ fractional part, i.e.,

$\operatorname{Integers}(\beta)=\left\{z=a_{-N+1} \beta^{N-1}+\cdots+a_{-1} \beta+a_{0} \mid N \geq 0, d_{\beta}\left(\beta^{-N} z\right)=a_{-N+1} \ldots a_{0} 0^{\infty}\right\}$.

Note that we obviously have the inclusion $\operatorname{Integers}(\beta) \subset \mathbb{Z}[\beta]$.

To understand the structure of $\operatorname{Integers}(\beta)$, Thurston [166], inspired by Rauzy [141], introduced a compact representation of the set Integers $(\beta)$. In order to define this representation note that if $x \in \mathbb{Z}[\beta]$, there exists a polynomial $P \in \mathbb{Z}[X]$ such that $x=P(\beta)$. Let $x^{(j)}=P\left(\beta^{(j)}\right)$ for $1 \leq j \leq n$. Then the Galois conjugates of $x$ are included in the the set $\left\{x^{(j)} ; 1 \leq j \leq n\right\}$ (notice that we may have $x^{\left(j_{1}\right)}=x^{\left(j_{2}\right)}$ for some indices $j_{1} \neq j_{2}$ in this set). The canonical representation of a $\beta$-integer is defined by

$$
\begin{aligned}
\Xi: \operatorname{Integers}(\beta) & \rightarrow \mathbb{R}^{r-1} \times \mathbb{C}^{s} \\
x & \mapsto\left(x^{(2)}, \ldots, x^{(r)}, x^{(r+1)}, \ldots, x^{(r+s)}\right)
\end{aligned}
$$


This embedding allows us to define the following tile associated to $\beta$-expansions.

Definition 2.11. - Let $\beta$ be a Pisot unit. The $\beta$-numeration tile is the closure of the set of $\beta$-integers under the canonical embedding $\Xi$, i.e.,

$$
\widetilde{\mathcal{J}}:=\overline{\Xi(\operatorname{Integers}(\beta))} \text {. }
$$

In order to establish a connection between $\beta$-numeration tiles and central tiles, let us introduce a suitable decomposition of $\tilde{\mathcal{J}}$. To this matter, we remark that the set $\operatorname{Integers}(\beta)$ is a discrete subset of $\mathbb{R}_{+}$. The regularity of $\operatorname{Integers}(\beta)$ is described by the following well-known property of $\beta$-integers.

Proposition $2.12([\mathbf{1 0}, \mathbf{1 6 6}])$. - Let $z \in \operatorname{Integers}(\beta)$ be given and let $z=$ $a_{-N+1} \beta^{N-1}+\cdots+a_{-1} \beta+a_{0}$ denote its $\beta$-expansion. Let $t_{1}^{*} \ldots t_{i}^{*}$, with $i \geq 0$, be the longest prefix of $d_{\beta}^{*}(1)$ which is a suffix of $a_{-N+1} \ldots a_{0}$. Then $z+T_{\beta}^{i}(1)$ is the successor of $z$ in $\operatorname{Integers}(\beta)$, i.e., the smallest element in $\operatorname{Integers}(\beta)$ that is strictly greater than $z$.

Notice that, by convention, the longest prefix can be empty, leading to $i=0$. The proof of this result relies on the fact that $1-T_{\beta}^{i}(1)$ can be expanded as $t_{1}^{*} \ldots t_{i}^{*}$. We refer to $[\mathbf{1 0}, \mathbf{1 6 6}]$ for details. Combined with the property that $d_{\beta}^{*}(1)$ is eventually periodic, this proposition yields that the real numbers $T_{\beta}^{i}(1)(i \geq 0)$ have $n$ different values and thus the set of distances between two consecutive points in $\operatorname{Integers}(\beta)$ is finite.

A natural decomposition of $\operatorname{Integers}(\beta)$ is now given by considering the distance between a point and its successor in $\operatorname{Integers}(\beta)$. For every $1 \leq i \leq n$, we define the subtile $\widetilde{\mathcal{T}(i)}$ of $\widetilde{\mathcal{T}}$ to be the closure of the set of the points $\Xi(z)$ with $z \in \operatorname{Integers}(\beta)$ such that the distance from $z$ to its successor in $\operatorname{Integers}(\beta)$ is equal to $T_{\beta}^{i-1}(1)$.

Definition 2.13. - Let $\beta$ be a Pisot unit and let $n$ and $m$ by given as in (2.10). Let $i \in\{1, \ldots, n\}$. The $\beta$-numeration subtile $\widetilde{\mathcal{T}(i)}$ is defined as the closure of the set of points $\Xi(z)$ such that $z \in \operatorname{Integers}(\beta)$ can be expanded as $z=a_{-N+1} \beta^{N-1}+\cdots+$ $a_{-1} \beta+a_{0}$ and the longest prefix $t_{1}^{*} \ldots t_{I}^{*}$ of $d_{\beta}^{*}(1)$ which is also a suffix of $a_{-N+1} \ldots a_{0}$ satisfies

— if $i=1$ then $t_{1}^{*} \ldots t_{I}^{*}=\varepsilon$,

- if $2 \leq i \leq m$ then $t_{1}^{*} \ldots t_{I}^{*}=t_{1}^{*} \ldots t_{i-1}^{*}$,

- if $m<i \leq n$ then $t_{1}^{*} \ldots t_{I}^{*}=t_{1}^{*} \ldots t_{m}^{*}\left(t_{m+1}^{*} \ldots t_{n}^{*}\right)^{\ell} t_{m+1}^{*} \ldots t_{i-1}^{*}$.

As detailed in $[\mathbf{1 1 8}, \mathbf{1 6 6}]$, each $\beta$-numeration subtile can be decomposed according to the last digit in the expansion of points of Integers $(\beta)$ contained in the tile. Using the characterization of $\beta$-expansions as those infinite words that are strictly smaller than $d_{\beta}^{*}(1)=t_{1}^{*} \ldots t_{m}^{*}\left(t_{m+1}^{*} \ldots t_{n}^{*}\right)^{\infty}$ yields that the $\beta$-numeration subtiles are the 
solution of the following set of GIFS equations (here the linear mapping $\mathbf{h}_{\text {diag }}: \mathbb{R}^{r-1} \times$ $\mathbb{C}^{s} \rightarrow \mathbb{R}^{r-1} \times \mathbb{C}^{s}$ is defined by $\left.\mathbf{h}_{\text {diag }}\left(x_{2}, \ldots, x_{r+s}\right)=\left(\beta^{(2)} x_{2}, \ldots, \beta^{(r+s)} x_{r+s}\right)\right)$.

$$
\begin{aligned}
\widetilde{\mathcal{J}(i)} & =\mathbf{h}_{\operatorname{diag}} \widetilde{\mathcal{J}(i-1)}+\Xi\left(t_{i-1}^{*}\right), i \in\{1, \ldots, n\} \backslash\{1, m+1\}, \\
\widetilde{\mathcal{J}(1)} & =\bigcup_{j=1}^{n} \int_{k=0}^{t_{j}^{*}-1}\left(\mathbf{h}_{\operatorname{diag}} \widetilde{\mathcal{J}(j)}+\Xi(k)\right), \\
\widetilde{\mathcal{J}(m+1)} & =\left(\mathbf{h}_{\operatorname{diag}} \widetilde{\mathcal{T}(m)}+\Xi\left(t_{m}^{*}\right)\right) \cup\left(\mathbf{h}_{\operatorname{diag}} \widetilde{\mathcal{J}(n)}+\Xi\left(t_{n}^{*}\right)\right)
\end{aligned}
$$

if $0<m<n$, i.e., if $d_{\beta}^{*}(1)$ has a pre-period, and

$$
\begin{aligned}
& \widetilde{\mathcal{J}(i)}=\mathbf{h}_{\text {diag }} \widetilde{\mathcal{J}(i-1)}+\Xi\left(t_{i-1}^{*}\right), i \in\{2, \ldots, n\} \\
& \widetilde{\mathcal{J}(1)}=\bigcup_{j=1}^{n} \bigcup_{k=0}^{t_{j}^{*}-1}\left(\mathbf{h}_{\operatorname{diag}} \widetilde{\mathcal{J}(j)}+\Xi(k)\right) \cup\left(\mathbf{h}_{\operatorname{diag}} \widetilde{\mathcal{J}(n)}+\Xi\left(t_{n}^{*}\right)\right)
\end{aligned}
$$

if $m=0$, i.e., if $d_{\beta}^{*}(1)$ has no pre-period.

Let a Pisot unit $\beta>1$ be given and assume that $d_{\beta}^{*}(1)$ is given as in (2.10). Now, let us introduce the so called $\beta$-substitution over the $n$-letter alphabet $\mathscr{G}=\mathscr{G}_{\beta}$. It is defined by

$$
\left\{\begin{array}{l}
\sigma_{\beta}(k)=1^{t_{k}^{*}}(k+1), \quad \text { if } 1 \leq k<n, \\
\sigma_{\beta}(n)=1^{t_{n}^{*}}(m+1) .
\end{array}\right.
$$

One can check easily that the prefix-suffix graph of the substitution $\sigma_{\beta}$ implies that the restrictions on the digit strings are governed by the improper expansion $d_{\beta}^{*}(1)$ of 1. We deduce that $\beta$ is its largest eigenvalue, that is, a Pisot number (this is not hard to prove; a short proof is contained in the master thesis $[\mathbf{1 6 3}$, p. 41]). Moreover, one easily calculates that the expanding left eigenvalue of the incidence matrix of $\sigma_{\beta}$ can be chosen to be

$$
\mathbf{v}_{\beta}=\left(1, T_{\beta}(1), \ldots, T_{\beta}^{n-1}(1)\right)
$$

(note that each of its entries is contained in $\mathbb{Z}[\beta]$ ).

For $\sigma_{\beta}$ the space $\mathbb{H}_{c}$ is isomorphic to $\mathbb{R}^{r-1} \times \mathbb{C}^{s}$ (each element of $\mathbb{R}^{r-1} \times \mathbb{C}^{s}$ corresponds to a set of coordinates along the eigendirections). Moreover, this isomorphism is a conjugacy between the contracting mappings $\mathbf{h}_{\text {diag }}$ and $\mathbf{h}$. As a specific example, the isomorphism maps $\Xi(k)$ to $\pi \mathbf{l}\left(1^{k}\right)$ for every $k \in \mathbb{N}$. With this correspondence at hand, one checks that the GIFS in (2.12) and (2.13) for the respective case is exactly the same as the one in (2.7) satisfied by the central tile of the $\beta$-substitution. By unicity of the solution of a GIFS, we conclude that $\beta$-numeration tiles as introduced in [166] form a special class of central tiles of substitutions (for more details see [45]).

Proposition 2.14 ([45]). - Let $\beta$ be a Pisot unit. Let $\sigma_{\beta}$ be the $\beta$-substitution defined from the $\beta$-expansion of 1 . Then the $\beta$-numeration subtile $\widetilde{\mathcal{T}(i)}$ is an affine image of the subtile $\mathcal{T}(i)$ associated with the substitution $\sigma_{\beta}$ for each $i \in\{1, \ldots, n\}$. 


\subsection{Dumont-Thomas numeration}

We have mentioned in the previous section that the central tile related to a $\beta$ substitution is a compact representation of those real numbers whose $\beta$-expansion has no fractional part. We detail now how this property can be extended to all substitutions.

Let $\sigma$ denote a primitive unit Pisot substitution. The notion of Dumont-Thomas numeration system generalizes the concept of beta-numeration and allows to expand nonnegative real numbers with respect to the combinatorics of a substitution [75]. The underlying idea is still to expand a positive real number number $x \in[0,1)$ as $x=\frac{\delta_{1}}{\beta}+\frac{\delta_{2}}{\beta^{2}}+\cdots$ (where $\beta$ is the Perron-Frobenius eigenvalue of $\sigma$ ), but to define the admissible digit strings in terms of $\sigma$. In particular, this requires to define a new set of digits $\mathscr{D}$ (instead of $\{1, \ldots,\lceil\beta\rceil-1\}$ ) together with an admissibility condition for expansions $\delta_{1} \delta_{2} \ldots$ in terms of $\sigma$.

Dumont and Thomas [74] proved that a natural way to define such expansions is given by the prefix-suffix graph. The set of digits is deduced from the labels of the prefix-suffix graph $\mathscr{P}$ (see Definition 2.5) as follows

$$
\mathscr{D}=\left\{\left\langle\mathbf{l}(p), \mathbf{v}_{\beta}\right\rangle ;(p, a, s) \in \mathscr{P}\right\} .
$$

Let $\sigma$ be a $\beta$-substitution $\sigma_{\beta}$. Then - provided that $\mathbf{v}_{\beta}$ is normalized as in (2.14) - we exactly recover $\mathscr{D}=\{1, \ldots,\lceil\beta\rceil-1\}$ since

- all prefixes in labels of the prefix-suffix graph have the shape $p=1^{\alpha}$ with $\alpha \leq t_{1}$

$-\left\langle\mathbf{l}(1), \mathbf{v}_{\beta}\right\rangle=1$ (since $\mathbf{v}_{\beta}$ the normalized as in (2.14)),

$-t_{1}=\lceil\beta\rceil-1$ (by the definition of the $\beta$-expansion),

- and $t_{k} \leq t_{1}$ for all $k>1$ (by the admissibility condition).

Dumont and Thomas were able to prove the following theorem (even in a slightly more general context than we state it here).

Theorem 2.15 ([75]). - Let $\sigma$ be a primitive unit Pisot substitution on the alphabet $\mathcal{G}$. Assume that the dominant eigenvalue of its incidence matrix is a Pisot number $\beta$. Let us fix $i \in \mathscr{G}$. For every real number $x \in\left[0,\left\langle\mathbf{e}_{i}, \mathbf{v}_{\beta}\right\rangle\right)$, there exists a unique reversed walk $\left(p_{k}, i_{k}, s_{k}\right)_{k \geq 1}$ in the prefix-suffix graph such that

- $\sigma(i)=p_{1} i_{1} s_{1}$

- there exist infinitely many nonempty suffixes in the sequence $\left(s_{k}\right)_{k \geq 1}$.

Then

$$
x=\frac{\left\langle\mathbf{l}\left(p_{1}\right), \mathbf{v}_{\beta}\right\rangle}{\beta}+\frac{\left\langle\mathbf{l}\left(p_{2}\right), \mathbf{v}_{\beta}\right\rangle}{\beta^{2}}+\cdots
$$

We call this expansion the $(\sigma, i)$-expansion of $x$. 
When $\sigma$ equals the $\beta$-substitution $\sigma_{\beta}$, one should notice that the condition in Theorem 2.15 is just the admissibility condition for $\beta$-expansions. Indeed, by the definition of $\sigma_{\beta}$, the sequence $\left(p_{k}, i_{k}, s_{k}\right)_{k \geq 1}$ is the labeling of a backwards walk in the prefix-suffix graph if and only if $p_{k}=1^{\alpha_{k}}$ where $\alpha_{1} \alpha_{2} \ldots$ is strictly smaller than $d_{\beta}^{*}(1)$, which easily leads to the admissibility condition.

Therefore, Theorem 2.15 can be seen as an extension of the admissibility condition of Parry to substitutions. Let us note that one may obtain a different type of numeration for each letter $i$. Nevertheless, one easily checks that the numeration associated with the letter 1 is exactly the $\beta$-numeration over $[0,1)$.

Dumont-Thomas numeration shares many properties with $\beta$-numeration. The $(\sigma, i)$-expansions can be computed thanks to a greedy algorithm coding the trajectories of a dynamical system [45]. Also, for every $i \in \mathscr{G}$, every element of $\mathbb{Q}(\beta) \cap\left[0,\left\langle\mathbf{e}_{i}, \mathbf{v}_{\beta}\right\rangle\right)$ admits an eventually periodic $(\sigma, i)$-expansion. Moreover, we can generalize $(\sigma, i)$ expansions to arbitrary elements $x \in \mathbb{R}^{+}$by permitting nonnegative powers of $\beta$ in (2.15). With this generalization, the set $\operatorname{Integers}(\sigma)$ is defined as the set of all positive real numbers having a $(\sigma, i)$-expansion in which only nonnegative powers of $\beta$ occur.

Finally, as we did in the framework of $\beta$-expansions, we can apply the mapping $\Xi$ defined in $(2.11)$ to finite $(\sigma, i)$-expansions. By doing this we associate to the expansion $x=\left\langle\mathbf{l}\left(p_{N-1}\right), \mathbf{v}_{\beta}\right\rangle \beta^{N-1}+\cdots+\left\langle\mathbf{l}\left(p_{-M}\right), \mathbf{v}_{\beta}\right\rangle \beta^{-M}$ the dual $(\sigma, i)$-expansion

$$
\Xi(x)=\mathbf{h}_{\text {diag }}^{N-1}\left(\Xi\left\langle\mathbf{l}\left(p_{N-1}\right), \mathbf{v}_{\beta}\right\rangle\right)+\cdots+\mathbf{h}_{\text {diag }}^{-M}\left(\Xi\left(\left\langle\mathbf{l}\left(p_{-M}\right), \mathbf{v}_{\beta}\right\rangle\right) .\right.
$$

We can extend this definition to infinite $(\sigma, i)$-expansions in a natural way. Indeed, a dual $(\sigma, i)$-expansion of an element $z \in \mathbb{R}^{r-1} \times \mathbb{C}^{s}$ is given by

$$
z=\mathbf{h}_{\operatorname{diag}}^{-N+1}\left(\Xi\left\langle\mathbf{l}\left(p_{N-1}\right), \mathbf{v}_{\beta}\right\rangle\right)+\mathbf{h}_{\operatorname{diag}}^{-N+2}\left(\Xi\left(\left\langle\mathbf{l}\left(p_{-N+2}\right), \mathbf{v}_{\beta}\right\rangle\right)+\cdots\right.
$$

where $\left(p_{k}, i_{k}, s_{k}\right)_{k \geq-N}$ is a walk in the prefix-suffix graph of $\sigma$. We refer to these expansions as dual expansions to the Dumont-Thomas numeration system, since we expand the elements of $\mathbb{R}^{r-1} \times \mathbb{C}^{s}$ by using ascending powers of $\beta$ (and not descending ones as in (2.15)), leading us to reverse the admissibility condition - and therefore, to read the prefix-suffix graph forward instead of backward as it is performed in the Dumont-Thomas numeration system.

Note that by the conjugacy between $\mathbf{h}$ and $\mathbf{h}_{\text {diag }}$ mentioned at the end of Section $2.5, \mathbf{h}$-ary representations of elements of $\mathbb{H}_{c}$ are conjugate to dual $(\sigma, i)$-expansions of elements of $\mathbb{R}^{r-1} \times \mathbb{C}^{s}$. In the present monograph we will work with $\mathbf{h}$-ary representations. In view of this conjugacy each result we prove for $\mathbf{h}$-ary representations will also hold for dual $(\sigma, i)$-expansions. In particular, we will see (in the cloth of h-ary representations) that the geometric property $(\mathrm{F})$ (see Definition 3.6) implies that each element of $\mathbb{R}^{r-1} \times \mathbb{C}^{s}$ admits a dual $(\sigma, i)$-expansion (see Proposition 3.9). Moreover, contrary to the Dumont-Thomas numeration system, we cannot always expect unicity of dual $(\sigma, i)$-expansions. We will be able to characterize points that do not have a unique dual $(\sigma, i)$ expansion. 
REMARK 2.16. - If we specialize to beta-substitutions, we end up in the betanumeration framework of the previous section. In particular, if $\sigma_{\beta}$ is the $\beta$ substitution of a cubic Pisot number $\beta$ with two complex conjugates $\alpha$ and $\bar{\alpha}$, the mapping $\Xi$ is much simpler since $\Xi(\beta)=\alpha$. Moreover, for each prefix $p_{k}=1^{a_{k}}$ we have $\Xi\left(\left\langle\mathbf{l}\left(p_{k}\right), \mathbf{v}_{\beta}\right\rangle\right)=\left\langle\mathbf{e}_{1}, \mathbf{v}_{\alpha}\right\rangle a_{k}$. In this case the $(\sigma, i)$-expansion of a complex number $z \in \mathbb{C}$ is

$$
z=\alpha^{-N+1} a_{-N+1}+\cdots+\alpha^{-1} a_{-1}+a_{0}+\alpha a_{1}+\cdots+\alpha^{k} a_{k}+\cdots
$$

where the digits $a_{k} \in\{0, \ldots,\lceil\beta\rceil-1\}$ satisfy an admissibility condition that is a reversed Parry admissibility condition. This can be considered as an $\alpha$-expansion of complex numbers and is studied for instance in $[\mathbf{1 0}, \mathbf{1 4 7}]$. Note that if the cubic Pisot number $\beta$ satisfies the so-called finiteness property $(\mathrm{F})$, each $z \in \mathbb{C}$ admits a $(\sigma, i)$-expansion $(2.16)$.

Finally, we can associate a tile to Dumont-Thomas numeration.

Definition 2.17. - Let $\sigma$ be a primitive unit Pisot substitution. The DumontThomas numeration tile is the the closure of the set Integers $(\sigma)$ under the canonical embedding $\Xi$, i.e.,

$$
\widetilde{\mathcal{J}}:=\overline{\Xi(\operatorname{Integers}(\sigma))}
$$

Dumont-Thomas numeration subtiles $\widetilde{\mathcal{T}(i)}(i \in \mathscr{C})$ of $\tilde{\mathcal{J}}$ can be defined by classifying the elements $x \in \operatorname{Integers}(\sigma)$ according to the state of the prefix-suffix graph in which the walk defining the $(\sigma, i)$-expansion of $x$ starts. According to the conjugacy between $\mathbf{h}$ and $\mathbf{h}_{\text {diag }}$ mentioned at the end of Section 2.5, Corollary 2.8 implies that each subtile $\mathcal{T}(i)$ of the central tile of $\sigma$ is an affine image of the subtile $\widetilde{\mathcal{T}(i)}$.

This relationship between dual expansions and central tiles enables us to use central tiles in order to prove the existence and the unicity of dual expansion in the forthcoming sections. 


\section{CHAPTER 3}

\section{MULTIPLE TILINGS INDUCED BY THE CENTRAL TILE AND ITS SUBTILES}

One interesting feature of the central tile $\mathcal{T}$ and its subtiles $\mathcal{T}(i)(i \in \mathscr{Q})$ is that they can tile the beta-contracting space $\mathbb{H}_{c}$ in different ways. Exploiting properties of these multiple tilings will allow us to study the boundary as well as topological properties of $\mathcal{T}$ and $\mathcal{T}(i)$. In the following definition we will make precise what we mean by a multiple tiling and by a tiling.

Definition $3.1([\mathbf{1 0 6}, \mathbf{1 0 9}, \mathbf{1 4 0}])$. - Let $\sigma$ be a primitive unit Pisot substitution. A multiple tiling of $\mathbb{H}_{c}$ by the subtiles $\mathcal{T}(i)(i \in \mathscr{Q})$ associated with $\sigma$ is given by a collection $\mathscr{I}=\{\mathcal{J}(i)+\gamma ;(\gamma, i) \in \Gamma\}$ (where $\Gamma \subset \mathbb{H}_{c} \times \mathscr{G}$ is the translation set) having the following properties.

1. $\mathbb{H}_{c}=\bigcup_{(\gamma, i) \in \Gamma} \mathcal{T}(i)+\gamma$.

2. Each compact subset of $\mathbb{H}_{c}$ intersects a finite number of tiles (this property is called local finiteness).

3. There is a positive integer $p$ such that almost all points in $\mathbb{H}_{c}$ (w.r.t. the $(d-1)$ dimensional Lebesgue measure) are covered exactly $p$ times by the elements of the collection 9 .

When distinct elements of $\mathscr{I}$ have pairwise nonintersecting interiors, i.e., if $p=1$, then the multiple tiling is a tiling.

For subtiles of central tiles, several multiple tilings can be defined. The principle is to project a subset of points of $\mathbb{Z}^{n}$ on the beta-contracting space $\mathbb{H}_{c}$. Depending on the properties of this subset, we get two different multiple tilings which will be discussed in Sections 3.1 and 3.2, respectively. For each primitive unit Pisot substitution these multiple tilings are conjectured to be tilings (see Section 3.3). We mention that this conjecture is not true in more general settings. For instance, Kalle and Steiner [102] were able to exhibit a multiple tiling with $p=2$ ("double tiling") which is defined by using symmetric $\beta$-expansions for $\beta$ equal to the dominant root of $X^{3}-X^{2}-X-1$. 


\subsection{The self-replicating multiple tiling}

3.1.1. The self-replicating translation set. - A first translation set that gives rise to a multiple tiling can be obtained by projecting on the beta-contracting space all the points with integer coordinates that approximate this space. The "discretization" stemming from this approximation corresponds to the notion of arithmetic space introduced in [142]; it consists in approximating the space $\mathbb{H}_{c}$ by selecting points $\mathbf{x}$ with integral coordinates that are above $\mathbb{H}_{c}$ but near enough that $\mathbf{x}$ shifted down by a canonical basis vector $\mathbf{e}_{i}$, say, is below $\mathbb{H}_{c}[\mathbf{2 8}]$.

Definition 3.2. - Let $\sigma$ be a primitive unit Pisot substitution over the alphabet $\mathscr{G}$. The self-replicating translation set is defined by

$$
\Gamma_{\text {srs }}:=\left\{[\pi(\mathbf{x}), i] \in \pi\left(\mathbb{Z}^{n}\right) \times \mathscr{Q} ; \quad 0 \leq\left\langle\mathbf{x}, \mathbf{v}_{\beta}\right\rangle<\left\langle\mathbf{e}_{i}, \mathbf{v}_{\beta}\right\rangle\right\} .
$$

A pair $[\pi(\mathbf{x}), i]$ is called face.

REMARK 3.3. - In the irreducible case, the term face can be justified as follows. Consider a face $[\gamma, i] \in \Gamma_{\text {srs }}$ as defined above. If the substitution is irreducible, the restriction of the mapping $\pi$ to $\mathbb{Z}^{n}$ is one-to-one by (2.5). In particular, if we have $[\pi(\mathbf{x}), i],[\pi(\mathbf{y}), i] \in \Gamma_{\text {srs }}$ with $\mathbf{x}, \mathbf{y} \in \mathbb{Z}^{n}$ satisfying $[\pi(\mathbf{x}), i]=[\pi(\mathbf{y}), i]$ then $\mathbf{x}=\mathbf{y}$. Consequently, there exists a unique $\mathbf{x} \in \mathbb{Z}^{n}$ such that $\gamma=\pi(\mathbf{x})$. Thus we can interpret $[\gamma, i]$ as the set

$$
\mathbf{x}+\left\{\theta_{1} \mathbf{e}_{1}+\cdots+\theta_{i-1} \mathbf{e}_{i-1}+\theta_{i+1} \mathbf{e}_{i+1}+\cdots+\theta_{n} \mathbf{e}_{n} ; \theta_{j} \in[0,1] \text { for } j \neq i\right\},
$$

which is the face orthogonal to the $i$-th canonical coordinate in a unit cube located in $\mathbf{x}$. One can show that this set of faces is the discrete approximation of the betacontracting space $\mathbb{H}_{c}(c f$. [28]). Moreover, the projections

$$
\pi(\mathbf{x})+\pi\left(\left\{\theta_{1} \mathbf{e}_{1}+\cdots+\theta_{i-1} \mathbf{e}_{i-1}+\theta_{i+1} \mathbf{e}_{i+1}+\cdots+\theta_{n} \mathbf{e}_{n} ; \theta_{j} \in[0,1] \text { for } j \neq i\right\}\right)
$$

have disjoint interior in $\mathbb{H}_{c}$ and they provide a polyhedral tiling of $\mathbb{H}_{c}$.

In the reducible case $\mathbb{H}_{c}$ is no longer a hyperplane, hence, the notion of discrete approximation by faces of cubes is not well defined in general (however, for special situations a similar kind of polygonal tiling of $\mathbb{H}_{c}$ can be defined even in the reducible case; see $[\mathbf{8 1}])$. In this case, the projections of faces do overlap. The restriction of the mapping $\pi$ to $\mathbb{Z}^{n}$ is not one-to-one. Nevertheless, if $[\pi(\mathbf{x}), i],[\pi(\mathbf{y}), i] \in \Gamma_{\text {srs }}$ with $\mathbf{x}, \mathbf{y} \in \mathbb{Z}^{n}$ satisfy $[\pi(\mathbf{x}), i]=[\pi(\mathbf{y}), i]$ then $\left\langle\mathbf{x}, \mathbf{v}_{\beta}\right\rangle=\left\langle\mathbf{y}, \mathbf{v}_{\beta}\right\rangle$ so that $\mathbf{y}-\mathbf{x} \in \mathbb{H}_{s}$.

3.1.2. The dual substitution $E_{1}^{*}$ and the geometric property $(\mathbf{F})$. - The set $\Gamma_{\text {srs }}$ is called self-replicating since it is stabilized by an inflation action on $\pi\left(\mathbb{Z}^{n}\right) \times \mathscr{C}$, obtained as the dual of the so-called one-dimensional realization $\mathbf{E}_{1}$ of $\sigma$ (see e.g. [30] for a definition of $\mathbf{E}_{1}$ ). This inflation action is defined as follows. 
Definition 3.4. - The dual of a substitution $\sigma$ is denoted by $\mathbf{E}_{1}^{*}$. It is defined on the set $\Pi\left(\pi\left(\mathbb{Z}^{n}\right) \times \mathscr{G}\right)$ of subsets of $\pi\left(\mathbb{Z}^{n}\right) \times \mathscr{G}$ by

$$
\begin{aligned}
& \mathbf{E}_{1}^{*}[\gamma, i]= \bigcup_{j \in \mathscr{Q}, \sigma(j)=p i s}\left[\mathbf{h}^{-1}(\gamma+\pi \mathbf{l}(p)), j\right] \in \Pi\left(\pi\left(\mathbb{Z}^{n}\right) \times \mathscr{G}\right) \\
& \text { and } \mathbf{E}_{1}^{*}\left(X_{1}\right) \cup \mathbf{E}_{1}^{*}\left(X_{2}\right)=\mathbf{E}_{1}^{*}\left(X_{1} \cup X_{2}\right) .
\end{aligned}
$$

The stabilization condition for $\mathbf{E}_{1}^{*}$ is contained in the following proposition.

Proposition $3.5([\mathbf{3 0}, \mathbf{8 1}])$. - Let $\sigma$ be a primitive unit Pisot substitution. Then the dual substitution $\mathbf{E}_{1}^{*}$ maps $\Gamma_{\text {srs }}$ onto $\Gamma_{\text {srs }}$. Moreover, for all $X_{1}, X_{2} \subseteq \pi\left(\mathbb{Z}^{n}\right) \times \mathscr{G}$ we have

$$
X_{1} \cap X_{2}=\varnothing \quad \Longrightarrow \quad \mathbf{E}_{1}^{*}\left(X_{1}\right) \cap \mathbf{E}_{1}^{*}\left(X_{2}\right)=\varnothing \text {. }
$$

It is easy to see that the set equation (2.7) can be rewritten by using the dual substitution. Indeed, we see directly from its definition that

$$
\forall i \in \mathscr{G}, \mathcal{T}(i)=\bigcup_{[\gamma, j] \in \mathbf{E}_{1}^{*}[\mathbf{0}, i]} \mathbf{h}(\mathcal{T}(j)+\gamma) .
$$

Iterating this for $m$ times yields the $m$-th subdivision of the subtiles as

$$
\forall i \in \mathscr{G}, \mathcal{T}(i)=\bigcup_{[\gamma, j] \in\left(\mathbf{E}_{1}^{*}\right)^{m}[\mathbf{0}, i]} \mathbf{h}^{m}(\mathcal{T}(j)+\gamma) .
$$

Thus the "addresses" of the subtiles occurring in the $m$-th subdivision of $\mathcal{J}(i)$ are given by the elements of $\left(\mathbf{E}_{1}^{*}\right)^{m}[\mathbf{0}, i]$. Denoting by $\beta$ the Perron-Frobenius eigenvalue of the prefix-suffix graph $\Gamma_{\sigma}$ of $\sigma$ we see that

$$
\beta^{m} \ll\left|\left(\mathbf{E}_{1}^{*}\right)^{m}[\mathbf{0}, i]\right| \ll \beta^{m}
$$

where $a_{m} \ll b_{m}$ means that there are constants $c>0, m_{0} \in \mathbb{N}$ such that $a_{m} \leq c b_{m}$ for all $m \geq m_{0}$.

For abbreviation let $\mathcal{U}$ denote the set of lower faces ("lower" in the obvious sense) of the unit cube of $\mathbb{R}^{n}$ at the origin, i.e.,

$$
\mathcal{U}:=\bigcup_{i \in \mathscr{C}}[\mathbf{0}, i] \subset \Gamma_{\mathrm{srs}} .
$$

An important property of $\mathbf{E}_{1}^{*}$ is that $\mathcal{U}$ is contained in $\mathbf{E}_{1}^{*}(\mathcal{U})(c f .[\mathbf{3 0}, \mathbf{8 1}])$. Hence, the sequence $\left(\left(\mathbf{E}_{1}^{*}\right)^{m}(\mathcal{U})\right)_{m \geq 0}$ is an increasing sequence of subsets of $\Gamma_{\text {srs }}$.

Definition 3.6 (Geometric property $(\mathrm{F})$ ). - Let $\sigma$ be a primitive unit Pisot substitution. If the iterations of $\mathbf{E}_{1}^{*}$ on $\mathcal{U}$ eventually cover the whole self-replicating translation set $\Gamma_{\mathrm{srs}}$, i.e., if

$$
\Gamma_{\mathrm{srs}}=\bigcup_{m \geq 0}\left(\mathbf{E}_{1}^{*}\right)^{m}(\mathcal{U})
$$

we say that the substitution satisfies the geometric property $(F)$. 
By expanding points using the definition of $\mathbf{E}_{1}^{*}$, this means that every point $[\gamma, i] \in$ $\Gamma_{\text {srs }}$ has a unique finite $\mathbf{h}$-ary representation

$$
\gamma=\mathbf{h}^{-m} \pi \mathbf{l}\left(p_{0}\right)+\cdots+\mathbf{h}^{-1} \pi \mathbf{l}\left(p_{m-1}\right)
$$

where $\left(p_{k}, i_{k}, s_{k}\right)_{0 \leq k \leq m-1}$ is the labelling of a finite walk in the prefix-suffix graph that ends at $i=i_{m}$. Even if the geometric property $(\mathrm{F})$ does not hold, the disjointness property in (3.3) implies that if $[\gamma, i] \in \Gamma_{\text {srs }}$ has a finite representation of the shape (3.8), then this representation is unique.

An analog of the geometric property (F) was introduced by Frougny and Solomyak [87] in the beta-numeration framework and further studied by Akiyama [8]. In the present form it is stated in [45]. There exist several sufficient conditions for the geometric property $(\mathrm{F})$ for specific classes of substitutions related to beta-numeration (see $[\mathbf{8}, \mathbf{3 1}, \mathbf{8 7}]$; we also mention the weaker property $(\mathrm{W})$ which is related to the tiling property of central tiles in the context of beta-numeration; cf. e.g. $[\mathbf{9}, \mathbf{1 8}])$. In one of our results we will relate the geometric property $(\mathrm{F})$ to topological properties of the central tile.

3.1.3. Definition of the self-replicating multiple tiling. - Now we shall use the self-replicating translation set to obtain a multiple tiling of $\mathbb{H}_{c}$ by the subtiles $\mathcal{T}(i)$. Before we state the result, recall that a Delaunay set is a uniformly discrete and relatively dense set. Moreover, by an aperiodic set we mean a discrete subset of $\mathbb{R}^{n}$ that is not invariant under any given set of $n$ linearly independent translations.

Proposition $3.7([\mathbf{3 1}, \mathbf{4 5}, \mathbf{4 8}, \mathbf{8 1}, \mathbf{1 0 1}])$. - Let $\sigma$ be a primitive unit Pisot substitution. The self-replicating translation set $\Gamma_{\mathrm{srs}}$ provides a multiple tiling $\{\mathcal{J}(i)+$ $\left.\gamma ;[\gamma, i] \in \Gamma_{\mathrm{srs}}\right\}$, that is

$$
\mathbb{H}_{c}=\bigcup_{[\gamma, i] \in \Gamma_{\mathrm{srs}}}(\mathcal{J}(i)+\gamma),
$$

where almost all points of $\mathbb{H}_{c}$ are covered $p$ times ( $p$ is a positive integer). The translation set $\Gamma_{\mathrm{srs}}$ is an aperiodic Delaunay set.

REMARK 3.8. - The multiple tiling $\left\{\mathcal{T}(i)+\gamma ;[\gamma, i] \in \Gamma_{\text {srs }}\right\}$ is called self-replicating multiple tiling (see for instance [109]).

Figure 3.1 contains an example for a self-replicating multiple tiling that is actually a tiling.

Note that (3.9), (3.8) and (2.8) imply the following result on $\mathbf{h}$-ary representations.

Proposition 3.9. - Let $\sigma$ be a primitive unit Pisot substitution satisfying the geometric property $(F)$. Then each $\gamma \in \mathbb{H}_{c}$ admits an $\mathbf{h}$-ary representation of the shape

$$
\gamma=\sum_{\ell=-m}^{\infty} \mathbf{h}^{\ell} \pi \mathbf{l}\left(p_{\ell}\right)
$$

where $\left(p_{\ell}, i_{\ell}, s_{\ell}\right)_{\ell \geq-m}$ is the labelling of a walk in the prefix-suffix graph $\Gamma_{\sigma}$. 


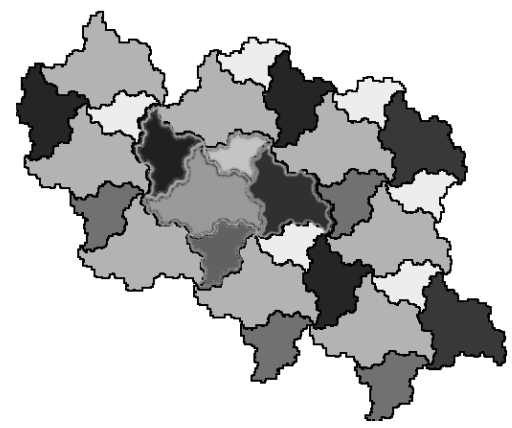

FiguRE 3.1. The self-replicating multiple tiling for the substitution $\sigma_{1}$ defined by $\sigma_{1}(1)=12, \sigma_{1}(2)=3, \sigma_{1}(3)=4, \sigma_{1}(4)=5, \sigma_{1}(5)=1$. We will see in Example 4.3 that the self-replicating multiple tiling induced by $\sigma_{1}$ is a tiling since $\sigma_{1}$ satisfies the tiling property which is defined in Definition 3.19.

\subsection{The lattice multiple tiling}

If $\sigma$ is an irreducible unit Pisot substitution, a discrete approximation of another hyperspace of $\mathbb{R}^{n}$ can be defined. Indeed, in this section we shall consider points with integral coordinates that lie on the antidiagonal hyperplane which is the hyperplane defined by the equation $\langle\mathbf{x},(1, \ldots, 1)\rangle=0$.

Definition 3.10. - Let $\sigma$ be an irreducible unit Pisot substitution on $n$ letters. The lattice translation set is defined by

$$
\Gamma_{\text {lat }}=\left\{[\gamma, i] \in \pi\left(\mathbb{Z}^{n}\right) \times \mathscr{G} ; \gamma \in \sum_{k=2}^{n} \mathbb{Z}\left(\pi\left(\mathbf{e}_{k}\right)-\pi\left(\mathbf{e}_{1}\right)\right)\right\} .
$$

The lattice translation set is obviously periodic.

Proposition 3.11 ([30, 60]). - Let $\sigma$ be an irreducible unit Pisot substitution that satisfies the strong coincidence condition. Then the collection $\left\{\mathcal{J}(i)+\gamma ;[\gamma, i] \in \Gamma_{\text {lat }}\right\}$ is a multiple tiling, that is

$$
\mathbb{H}_{c}=\bigcup_{[\gamma, i] \in \Gamma_{\text {lat }}}(\mathcal{T}(i)+\gamma)
$$

where almost all points are covered exactly $p$ times by this union $(p \in \mathbb{N})$.

Proof. - Consider the quotient mapping $\varphi$ from $\mathbb{H}_{c}$ to the $(d-1)$-dimensional torus $\mathbb{T}=\mathbb{H}_{c} / \mathscr{L}$, where $\mathcal{L}$ denotes the lattice $\mathscr{L}=\sum_{k=2}^{n} \mathbb{Z}\left(\pi\left(\mathbf{e}_{k}\right)-\pi\left(\mathbf{e}_{1}\right)\right)$.

We first prove that the union in (3.11) forms a covering of $\mathbb{H}_{c}$. This is equivalent with proving that the central tile maps to the full torus, that is, $\varphi(\mathcal{T})=\mathbb{T}$. First notice that the set $\left\{\varphi\left(\pi\left(\mathbf{e}_{i}\right)\right) ; 1 \leq i \leq n\right\}$ contains a single point, say $\mathbf{t}$. This follows from the definition of the quotient mapping $\varphi$. 


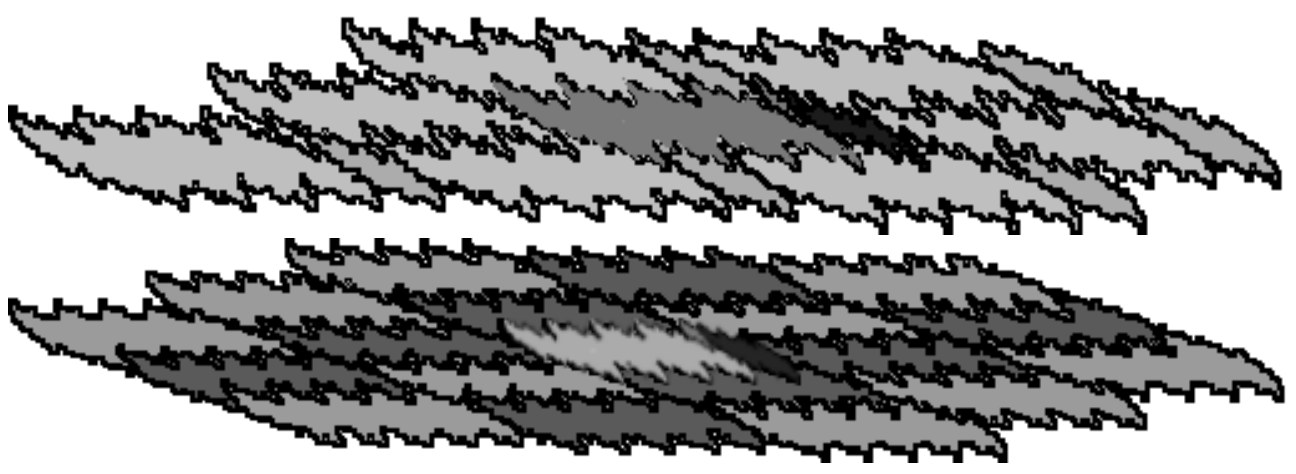

FiguRE 3.2. Self-replicating multiple tiling and lattice multiple tiling for the irreducible substitution $\sigma_{4}(1)=11112, \sigma_{4}(2)=11113$ and $\sigma_{4}(3)=1$. We will see in Example 4.3 that $\sigma_{4}$ satisfies the tiling property. Thus the self-replicating and lattice multiple tilings are tilings.

Let $u_{0} u_{1} \cdots \in \mathscr{G}^{\mathbb{N}}$ denote a one-sided periodic point of $\sigma$. By the definition of the central tile $\mathcal{T}$, we have $\varphi(\mathcal{T})=\overline{\left\{\varphi\left(\mathbf{l}\left(u_{0} \ldots u_{k-1}\right)\right) ; k \in \mathbb{N}\right\}}=\overline{\{k \mathbf{t} ; k \in \mathbb{N}\}}$ in the torus $\mathbb{T}$. To achieve the proof it remains to show by algebraic considerations that the addition of $\mathbf{t}$ on the torus is minimal: to this matter the Kronecker theorem can be applied after a precise study of the dependency between $\mathbf{t}$ and the projection $\varphi$. Hence, $\varphi(\mathcal{T})=\mathbb{T}$ which is equivalent to the covering property in (3.11). The fact that we get a multiple tiling follows from the minimality of the rotation by $\mathbf{t}$.

Details can be found in $[\mathbf{3 0 , 6 0}]$; see also the proof of Proposition 3.15 in Chapter 7.

REMARK 3.12. - Another way to prove that the union in (3.11) forms a covering of $\mathbb{H}_{c}$ runs as follows. Take a two-sided periodic point of $\sigma$ and consider the broken line associated to it. It is easy to see that the translates of the broken line by all points $\gamma$ with $[\gamma, i] \in \Gamma_{\text {lat }}$ meet each point of $\mathbb{Z}^{n}$. Since the projection $\pi\left(\mathbb{Z}^{n}\right)$ is dense in $\mathbb{H}_{c}$ (see the argument in [7, Proposition 1]) we get that the union in (3.11) is dense in $\mathbb{H}_{c}$. However, as this union is a locally finite union of compact sets we are done.

EXAMPLE 3.13. - The substitution $\sigma_{4}$ is irreducible. Its self-replicating multiple tiling as well as its lattice multiple tiling which are actually tilings (see Example 4.3) is depicted in Figure 3.2.

To generalize Proposition 3.11 to the reducible case we need to exhibit a suitable projection $\varphi$ onto a $(d-1)$-dimensional torus such that $\left\{\varphi\left(\pi\left(\mathbf{e}_{i}\right)\right) ; 1 \leq i \leq n\right\}$ contains a single point. This is possible if the the following condition, which seems to be new, is satisfied.

DEFINITION 3.14 (Quotient mapping condition). - Let $\sigma$ be a primitive unit Pisot substitution on $n$ letters. Let $d$ denote the degree of its dominant eigenvalue $\beta$. We 
say that $\sigma$ satisfies the quotient mapping condition if there exist $d$ distinct letters $B(1), \ldots, B(d)$ in $\mathscr{C}$ such that

$$
\forall i \in\{1, \ldots, n\} \quad\left\langle\mathbf{e}_{i}-\mathbf{e}_{B(1)}, \mathbf{v}_{\beta}\right\rangle \in \sum_{k \in\{2, \ldots, d\}} \mathbb{Z}\left\langle\mathbf{e}_{B(k)}-\mathbf{e}_{B(1)}, \mathbf{v}_{\beta}\right\rangle .
$$

If the quotient mapping condition holds, the lattice translation set is defined by

$$
\Gamma_{\text {lat }}=\left\{[\gamma, i] \in \pi\left(\mathbb{Z}^{n}\right) \times \mathscr{G} ; \gamma \in \sum_{k=2}^{d} \mathbb{Z}\left(\pi\left(\mathbf{e}_{B(k)}\right)-\pi\left(\mathbf{e}_{B(1)}\right)\right)\right\} .
$$

Under this condition (which is trivially satisfied if $\sigma$ is irreducible) the results of Proposition 3.11 also hold in the case of reducible primitive unit Pisot substitutions.

Proposition 3.15. - Let $\sigma$ be a primitive unit Pisot substitution that satisfies the strong coincidence condition and the quotient mapping condition. Then the collection $\left\{\mathcal{T}(i)+\gamma ;[\gamma, i] \in \Gamma_{\text {lat }}\right\}$ is a multiple tiling, i.e., (3.11) is satisfied where almost all points are covered exactly $p$ times by this union $(p \in \mathbb{N})$.

Proof. - Let $\mathscr{L}:=\sum_{k=2}^{d} \mathbb{Z}\left(\pi\left(\mathbf{e}_{B(k)}\right)-\pi\left(\mathbf{e}_{B(1)}\right)\right)$. If the quotient mapping condition is satisfied, we get

$$
\pi\left(\mathbf{e}_{i}\right) \equiv \pi\left(\mathbf{e}_{B(1)}\right) \quad(\bmod \mathscr{L}) \quad(1 \leq i \leq n) .
$$

Hence, the quotient mapping $\varphi$ from $\mathbb{H}_{c}$ to $\mathbb{H}_{c} / \mathscr{L}$ maps $\left\{\pi\left(\mathbf{e}_{i}\right) ; 1 \leq i \leq n\right\}$ to a single point t. The quotient mapping condition also implies that $\left\langle\mathbf{e}_{B(1)}, \mathbf{v}_{\beta}\right\rangle,\left\langle\mathbf{e}_{B(2)}, \mathbf{v}_{\beta}\right\rangle$, $\ldots,\left\langle\mathbf{e}_{B(d)}, \mathbf{v}_{\beta}\right\rangle$ are rationally independent so that the addition of $\mathbf{t}$ is minimal, which yields the result. A detailed proof is given in Chapter 7 .

EXAMPLE 3.16. - The substitution $\sigma_{1}(1)=12, \sigma_{1}(2)=3, \sigma_{1}(3)=4, \sigma_{1}(4)=5$, $\sigma_{1}(5)=1$ does not satisfy the quotient mapping condition. Indeed, if the quotient mapping condition is satisfied, the elements $\left\langle\mathbf{e}_{B(1)}, \mathbf{v}_{\beta}\right\rangle,\left\langle\mathbf{e}_{B(2)}, \mathbf{v}_{\beta}\right\rangle$ and $\left\langle\mathbf{e}_{B(3)}, \mathbf{v}_{\beta}\right\rangle$ are rationally independent. In this example we have $\mathbf{v}_{\beta}=\left(1, \beta-1, \beta^{2}-\beta,-\beta^{2}+\right.$ $\left.\beta+1, \beta^{2}-1\right)$. Then $\left\langle-\mathbf{e}_{1}+\mathbf{e}_{3}+\mathbf{e}_{4}, \mathbf{v}_{\beta}\right\rangle=0$ and $\left\langle\mathbf{e}_{2}+\mathbf{e}_{3}-\mathbf{e}_{5}, \mathbf{v}_{\beta}\right\rangle=0$. The first relation yields $\left\langle\mathbf{e}_{4}, \mathbf{v}_{\beta}\right\rangle=\left\langle\mathbf{e}_{1}, \mathbf{v}_{\beta}\right\rangle-\left\langle\mathbf{e}_{3}, \mathbf{v}_{\beta}\right\rangle$. Hence, if $\{B(1), B(2), B(3)\}$ contains $\{1,3\}$ we deduce that $\left\langle\mathbf{e}_{4}, \mathbf{v}_{\beta}\right\rangle$ belongs to the set on the right hand side of (3.12). If the quotient mapping condition holds this set must contain also $\left\langle\mathbf{e}_{4}-\mathbf{e}_{1}, \mathbf{v}_{\beta}\right\rangle$. Hence, also $\left\langle\mathbf{e}_{1}, \mathbf{v}_{\beta}\right\rangle$ belongs to this set. Assume w.l.o.g. that $B(1)=1$. Then we get $\left\langle\mathbf{e}_{1}, \mathbf{v}_{\beta}\right\rangle=a_{1}\left\langle\mathbf{e}_{1}-\mathbf{e}_{B(2)}, \mathbf{v}_{\beta}\right\rangle+a_{2}\left\langle\mathbf{e}_{1}-\mathbf{e}_{B(3)}, \mathbf{v}_{\beta}\right\rangle$ for certain integers $a_{1}, a_{2}$. Thus $\left(1-a_{1}-a_{2}\right)\left\langle\mathbf{e}_{1}, \mathbf{v}_{\beta}\right\rangle+a_{1}\left\langle\mathbf{e}_{B(2)}, \mathbf{v}_{\beta}\right\rangle+a_{2}\left\langle\mathbf{e}_{B(3)}, \mathbf{v}_{\beta}\right\rangle=0$. Since $a_{1}$, $a_{2}$ and $1-a_{1}-a_{2}$ cannot vanish simultaneously this implies that there must be a rational dependency between the elements $\left\langle\mathbf{e}_{B(1)}, \mathbf{v}_{\beta}\right\rangle,\left\langle\mathbf{e}_{B(2)}, \mathbf{v}_{\beta}\right\rangle,\left\langle\mathbf{e}_{B(3)}, \mathbf{v}_{\beta}\right\rangle$, a contradiction. With similar arguments we prove that $\{1,4\},\{2,5\}$ and $\{3,5\}$ cannot be subsets of $\{B(1), B(2), B(3)\}$. Thus the only remaining possibility is $\{B(1), B(2), B(3)\}=$ $\{2,3,4\}$. However, if the quotient mapping condition is satisfied for this set then $2-\beta=\left\langle\mathbf{e}_{1}, \mathbf{v}_{\beta}\right\rangle-\left\langle\mathbf{e}_{2}, \mathbf{v}_{\beta}\right\rangle$ must be an integer combination of $\left\langle\mathbf{e}_{3}-\mathbf{e}_{2}, \mathbf{v}_{\beta}\right\rangle=\beta^{2}-2 \beta+1$ 


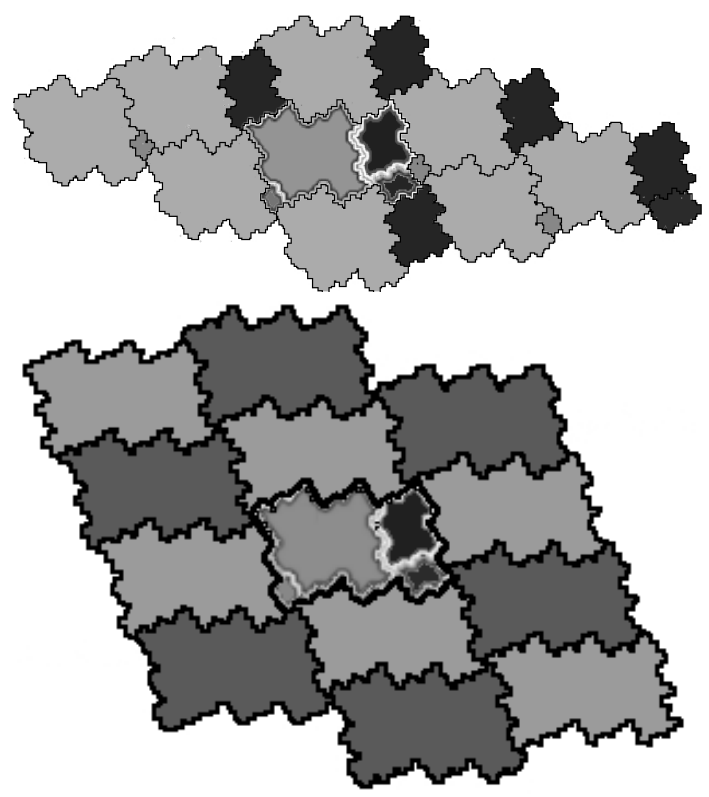

Figure 3.3. Self-replicating and lattice multiple tilings for the substitution $\sigma_{0}$. In the lattice multiple tiling, the subtiles are shown only in the central tile. Subdivision in subtiles is omitted in the other copies of the central tile.

Since $\sigma_{0}$ has the tiling property (see Definition 3.19), the self-replicating multiple tiling is a tiling. The same is true for the lattice multiple tiling (see Example 4.3).

and $\left\langle\mathbf{e}_{4}-\mathbf{e}_{2}, \mathbf{v}_{\beta}\right\rangle=-\beta^{2}+2$. As this is impossible since $\beta$ is a cubic irrational, we get a contradiction. Hence, the quotient mapping condition is not satisfied for $\sigma_{1}$.

EXAMPLE 3.17. - The substitution $\sigma_{0}(1)=112, \sigma_{0}(2)=113, \sigma_{0}(3)=4, \sigma_{0}(4)=1$ satisfies the quotient mapping condition. Indeed, we have $\mathbf{v}_{\beta}=\left(1, \beta-2, \beta^{2}-2 \beta-\right.$ $\left.2, \beta^{2}-3 \beta+1\right)$. Hence, $\left\langle\mathbf{e}_{4}-\mathbf{e}_{1}, \mathbf{v}_{\beta}\right\rangle=\beta^{2}-3 \beta=\beta^{2}-2 \beta-3-(\beta-3)=\left\langle\mathbf{e}_{3}-\right.$ $\left.\mathbf{e}_{1}, \mathbf{v}_{\beta}\right\rangle-\left\langle\mathbf{e}_{2}-\mathbf{e}_{1}, \mathbf{v}_{\beta}\right\rangle$. Thus a suitable set of letters $\{B(1), B(2), B(3)\}$ for the quotient mapping condition is given by $B(1)=1, B(2)=2, B(3)=3$. The self-replicating multiple tiling and the lattice multiple tiling (which are actually tilings in this case; see Example 4.3) are depicted in Figure 3.3.

REMARK 3.18. - It remains to characterize all reducible primitive unit Pisot substitutions that satisfy the quotient mapping condition. It is not clear whether this is possible by means of a simple criterion. 


\subsection{The tiling property and the Pisot conjecture}

Let $\sigma$ be a primitive unit Pisot substitution. A fundamental question is whether the self-replicating multiple tiling defined in Section 3.1 is indeed a tiling. This motivates the following definition.

Definition 3.19. - Let $\sigma$ be a primitive unit Pisot substitution. If the selfreplicating multiple tiling associated with $\sigma$ is a tiling we say that $\sigma$ has the tiling property.

It is conjectured that each primitive unit Pisot substitution has the tiling property. This conjecture is often called Pisot conjecture.

Ito and Rao [101] proved that if $\sigma$ is irreducible then the self-replicating multiple tiling is a tiling if and only if the same is true for the lattice multiple tiling. In the case of reducible substitutions the situation is different. Indeed, if a reducible primitive unit Pisot substitution does not satisfy the quotient mapping condition of Definition 3.14 the lattice multiple tiling cannot be properly defined. Thus a reasonable generalization of the Pisot conjecture to lattice tilings in the reducible case reads as follows. If a substitution satisfies the quotient mapping condition, then the lattice multiple tiling is a tiling.

There exists a variety of conditions which are equivalent to the tiling property of a substitution $\sigma$. We mention for instance the super-coincidence condition. This condition is defined for irreducible unit Pisot substitutions in Baker et al. [31] as well as in Ito and Rao [101]. A generalization of this condition to the reducible case can be found in $[\mathbf{8 1}]$. The main result concerning the super-coincidence condition is contained in the following theorem.

TheOREM 3.20 ([31, 48, 81, 101]). - Let $\sigma$ be a primitive unit Pisot substitution. Then the self-replicating multiple tiling is a tiling if and only if $\sigma$ satisfies the supercoincidence condition.

If $\sigma$ is irreducible, the lattice multiple tiling is a tiling if and only if $\sigma$ satisfies the super-coincidence condition.

Besides the super-coincidence condition there exist many other conditions that can be shown to be related to the tiling property. A survey of these conditions together with a thorough discussion of their relations to each other is given in [48].

In the irreducible case, the multiple lattice tiling has been extensively studied and presents many interesting features related to the symbolic dynamical system associated with the underlying substitution $[\mathbf{3 0}, \mathbf{8 6}, \mathbf{1 4 1}]$. As a consequence of the proof of Proposition 3.11, if the lattice multiple tiling is a tiling, then the substitutive dynamical system associated with $\sigma$ has a pure discrete spectrum, since it is measure theoretically conjugate to a toral translation.

The tiling condition has also been investigated in the framework of betanumeration. This viewpoint has been thoroughly explored and several tiling 
conditions are available. The best-known is the so-called property (W) $[\mathbf{9}, \mathbf{9 3}]$. Property $(\mathrm{W})$ is equivalent to the tiling property for the case of beta-substitutions. 


\section{CHAPTER 4}

\section{STATEMENT OF THE MAIN RESULTS: TOPOLOGICAL PROPERTIES OF CENTRAL TILES}

In this chapter we will state our main results in a slightly informal way. All details will be given in Chapter 6 where we also give the full proofs of all results. We do it that way in order to provide those readers who want to apply our results a way to use them without having to go into technical details.

\subsection{A description of specific subsets of the central tile}

We start with some notions and definitions needed in order to state the results. In all what follows we assume that $\sigma$ is a primitive unit Pisot substitution. We build several graphs to describe with GIFSs the intersections of tiles. Technical details and precise definitions will be given in Chapter 5. The main idea is the following: we intend to describe the intersection between two tiles $\mathcal{T}(i)$ and $\mathcal{T}(j)+\gamma$ for some $\gamma \in \mathbb{H}_{c}$. To do so, we consider the GIFS decomposition of each tile, that is $\mathcal{J}(i)=$ $\bigcup_{\sigma\left(i_{1}\right)=p_{1} i s_{1}} \mathbf{h} \mathcal{T}\left(i_{1}\right)+\pi \mathbf{l}\left(p_{1}\right)$ and $\mathcal{T}(j)=\bigcup_{\sigma\left(j_{1}\right)=p_{2} j s_{2}} \mathbf{h} \mathcal{T}\left(j_{1}\right)+\pi \mathbf{l}\left(p_{2}\right)$. Then we write a decomposition of the intersection as

$$
\mathcal{T}(i) \cap(\mathcal{J}(j)+\gamma)=\bigcup_{\substack{\sigma\left(i_{1}\right)=p_{1} i s_{1} \\ \sigma\left(j_{1}\right)=p_{2} j s_{2}}}\left(\mathbf{h} \mathcal{T}\left(i_{1}\right)+\pi \mathbf{l}\left(p_{1}\right)\right) \cap\left(\mathbf{h} \mathcal{T}\left(j_{1}\right)+\pi \mathbf{l}\left(p_{2}\right)+\gamma\right) .
$$

We express each element of this decomposition as the image of a translated intersection of tiles by $\mathbf{h}$, i.e.,

$$
\mathcal{T}(i) \cap(\mathcal{T}(j)+\gamma)=\bigcup_{\substack{\sigma\left(i_{1}\right)=p_{1} i_{1} \\ \sigma\left(j_{1}\right)=p_{2} j s_{2}}} \mathbf{h}\left(\mathcal{T}\left(i_{1}\right) \cap(\mathcal{T}\left(j_{1}\right)+\underbrace{\mathbf{h}^{-1} \pi \mathbf{l}\left(p_{2}\right)-\mathbf{h}^{-1} \pi \mathbf{l}\left(p_{1}\right)+\mathbf{h}^{-1} \gamma}_{=\gamma_{1}})\right)+\pi \mathbf{l}\left(p_{1}\right) .
$$

This equation means that the intersection between two tiles can be expressed as the union of intersections between other tiles. Let us denote the intersection $\mathcal{J}(i) \cap$ 
$(\mathcal{T}(j)+\gamma)$ by $B[i, \gamma, j]$. Then we have

$$
B[i, \gamma, j]=\bigcup_{\substack{\sigma\left(i_{1}\right)=p_{1} i s_{1}, \sigma\left(j_{1}\right)=p_{2} j s_{2} \\ \gamma_{1}=\mathbf{h}^{-1} \pi \mathbf{l}\left(p_{2}\right)-\mathbf{h}^{-1} \pi \mathbf{l}\left(p_{1}\right)+\mathbf{h}^{-1} \gamma}} \mathbf{h} B\left[i_{1}, \gamma_{1}, j_{1}\right]+\pi \mathbf{l}\left(p_{1}\right) .
$$

Now we build a graph with nodes $[i, \gamma, j]$ such that there exists an edge between $[i, \gamma, j]$ and $\left[i_{1}, \gamma_{1}, j_{1}\right]$ if a translate of $\mathbf{h} B\left[i_{1}, \gamma_{1}, j_{1}\right]$ appears in the decomposition of $B[i, \gamma, j]$. Starting from a certain finite set of nodes, we will prove that this graph is finite. Hence, a node $[i, \gamma, j]$ is the starting point of an infinite walk in this graph if and only if the intersection $B[i, \gamma, j]=\mathcal{T}(i) \cap(\mathcal{T}(j)+\gamma)$ is nonempty.

Depending on the purpose, we use different sets of initial nodes, and we use a similar process to describe intersections of more than two tiles, so that we finally define several different graphs. Besides graphs of this kind, several other types of graphs will be used. We want to give a short survey over all these graphs in the following list.

Zero-expansion graph: The nodes of the zero-expansion graph correspond to tiles $\mathcal{J}(i)+\gamma$ in the self-replicating tiling that contain $\mathbf{0}$.

Connectivity graphs : The tile-connectivity graph contains an edge between two letters $i, j \in \mathscr{G}$ if and only if $\mathcal{T}(i)$ and $\mathcal{T}(j)$ intersect.

For each letter $i$, the tile-refinement-connectivity graph of $\mathcal{J}(i)$ exhibits intersections between the subtiles that appear in the GIFS decomposition of $\mathcal{T}(i)$. Similarly, the boundary-connectivity graph of $\mathcal{J}(i)$ contains a node for each piece $\mathcal{J}(i) \cap(\mathcal{T}(j)+\gamma)$ with $[\gamma, i] \in \Gamma_{\text {srs }}$ that is nonempty and an edge between each two pieces that have nonempty intersection.

Moreover, the boundary-refinement-connectivity graph of a piece $\mathcal{T}(i) \cap(\mathcal{J}(j)+\gamma)$ exhibits intersections between the pieces that appear in the GIFS decomposition (4.2) of $\mathcal{T}(i) \cap(\mathcal{T}(j)+\gamma)$.

Self-replicating (SR) and lattice boundary graph : A point is called a double point if it is contained in a subtile of the central tile and in at least one other tile of a multiple tiling (self-replicating tiling or lattice tiling). The structure of the double-points in each of the above-mentioned tilings can be described by a GIFS governed by a graph. These graphs are called SR-boundary graph and lattice boundary graph, respectively.

$S R$-Contact graph : This $S R$-contact graph is also related to intersections of two tiles in the self-replicating lattice tiling. If $\sigma$ satisfies the tiling property, the boundary of the tiles $\mathcal{T}(i)(i \in \mathscr{C})$ can be written as a GIFS in terms of this graph.

Triple point and quadruple point graph : A point is called triple point (quadruple point, respectively) if it is contained in a subtile of the central tile as well as in at least two (three, respectively) other tiles of a multiple tiling. The triple (quadruple, respectively) points of the above mentioned self-replicating multiple tiling are the solution of a GIFS directed by the so-called triple point graph (quadruple point graph, respectively). 
Now we are ready to state our main results.

\subsection{Tiling properties of the central tile and its subtiles}

The SR-contact graph and the boundary graphs allow to check that the selfreplicating or lattice multiple tilings are indeed tilings.

THEOREM 4.1. - Let $\sigma$ be a primitive unit Pisot substitution over the alphabet $\mathscr{C}$ and let $\beta$ be the dominant eigenvalue of its incidence matrix. Let $\mu$ and $\mu_{\text {lat }}$ be the modulus of the largest eigenvalue of the incidence matrix of the SR-boundary graph $\mathscr{G}_{\mathrm{srs}}^{(B)}$ and the lattice boundary graph $\mathscr{G}_{\text {lat }}^{(B)}$, respectively. Then the following assertions hold.

(1) The self-replicating multiple tiling is a tiling if and only if $\mu<\beta$.

(2) If $\sigma$ is irreducible, the lattice multiple tiling is a tiling if and only if $\mu<\beta$.

(3) If the quotient mapping condition is satisfied, the lattice multiple tiling is a tiling if and only if $\mu_{\text {lat }}<\beta$.

Although the SR-contact graph does not exist for all reducible primitive Pisot substitutions it is a useful tool to check the tiling property, since it is often much smaller than the SR-boundary graph. We thus provide the following criterion. It can be proved in a similar way as Theorem 4.1 .

TheOREM 4.2. - Let $\sigma$ be an irreducible unit Pisot substitution over the alphabet $\mathscr{G}$. Let $\beta$ be the dominant eigenvalue of its incidence matrix and let $\mu^{\prime}$ be the modulus of the largest eigenvalue of the incidence matrix of the SR-contact graph $\mathscr{G}_{\mathrm{srs}}^{(C)}$. Then the following assertions hold.

(1) The self-replicating multiple tiling is a tiling if and only if $\mu^{\prime}<\beta$.

(2) The lattice multiple tiling is a tiling if and only if $\mu^{\prime}<\beta$.

Theorem 4.1 and Theorem 4.2 are effective for checking the tiling property as well as its violation, since the boundary graphs as well as the SR-contact graph can be constructed in finite time. Siegel [155] gives a criterion for the tiling property in terms of a graph which is related to the lattice boundary graph. However, this criterion is of a different flavor than Theorem 4.1.

Another way to check whether the self-replicating multiple tiling is a tiling consists in checking the super-coincidence condition. More precisely, the super-coincidence condition cannot be checked directly but the so called balanced pair algorithm ( $c f$. [48, $\mathbf{1 1 5}, \mathbf{1 5 7}]$ ) terminates with coincidences if and only if this condition is satisfied. In [48] many other methods for checking the tiling property are provided.

In the irreducible case, the lattice multiple tiling is a tiling if and only if the selfreplicating multiple tiling is a tiling (see [101]). Thus in each of the Theorems 4.1 and 4.2 Assertion (2) is an immediate consequence of Assertion (1). As for the reducible case, Ei and Ito [80] give examples of lattice tilings for some substitutions. Theorem 4.1 provides a new general criterion for lattice tilings in the reducible case. 
EXAMPLE 4.3. - The substitutions described in Section 2.4 have the following tiling properties.

- The substitution $\sigma_{0}$ generates both, a self-replicating and a lattice tiling. Both tilings are shown in Figure 3.3. A detailed treatment of the tiling properties of $\sigma_{0}$ is given in Example 5.11.

- The substitution $\sigma_{1}(1)=12, \sigma_{1}(2)=3, \sigma_{1}(3)=4, \sigma_{1}(4)=5, \sigma_{1}(5)=1$ generates a self-replicating tiling (see Figure 3.1) but not a lattice tiling. It does not satisfy the quotient mapping condition. To prove the self-replicating tiling property, details can be done in the same way as in the case of $\sigma_{0}$.

- Each of the substitutions $\sigma_{2}, \ldots, \sigma_{6}$ induces a self-replicating and a lattice tiling. This can be shown in the same way as it is shown for $\sigma_{0}$ in Example 5.11.

\subsection{Dimension of the boundary of central tiles}

A natural question is how to compute the Hausdorff and box-counting dimension of the boundary of $\mathcal{T}(i)$ (for a definition of these notions of dimension see e.g. Falconer [83]). This question was handled for the Tribonacci substitution in $[\mathbf{9 7 ,} \mathbf{1 2 7}]$. Moreover, in [84] an upper bound for the Hausdorff dimension is computed for a class of substitutions. Finally, [167] exhibits a formula for the box-counting dimension of a central tile in the irreducible case. This box-counting dimension coincides with the Hausdorff dimension when the contraction $\mathbf{h}$ on the contracting plane $\mathbb{H}_{c}$ is a similarity, that is, when the conjugates of $\beta$ all have the same modulus. If this is not the case, the calculation of the Hausdorff dimension of the boundary seems to be a very hard problem.

We include a slightly more general version of [167, Proposition 5.7 and Theorem 5.9]. Indeed, our version also contains the case of reducible substitutions.

THEOREM 4.4. - Let $\sigma$ be a primitive unit Pisot substitution over the alphabet $\mathscr{G}$. Let $\beta$ be the dominant eigenvalue of its incidence matrix and $\beta^{\prime}$ be one of the smallest conjugates of $\beta$ in modulus. Let $\mu$ denote the largest eigenvalue in modulus of the $S R$-boundary graph $^{(1)}$ of $\sigma$.

Suppose that the SR-boundary graph of $\sigma$ is strongly connected and $\mu<\beta$. Then the box-counting dimension of the boundary of $\mathcal{T}$ satisfies

$$
\forall i=1, \ldots, n, \quad \operatorname{dim}_{B}(\partial \mathcal{T})=\operatorname{dim}_{B}(\partial \mathcal{T}(i))=d-1+\frac{\log \beta-\log \mu}{\log \left|\beta^{\prime}\right|} .
$$

If all the conjugates of $\beta$ have the same modulus, then the box-counting dimensions in (4.3) agree with the Hausdorff dimensions.

(1) It is possible to use the SR-contact graph instead of the SR-boundary graph here. The SR-contact graph is often much smaller than the SR-boundary graph and therefore more convenient for calculations. However, the SR-contact graph in general does not exist for reducible substitutions (see Section 5.4). 
EXAMPLE 4.5. - In this example we deal with the box-counting dimension of the boundaries of $\mathcal{J}$ as well as $\mathcal{J}(i)(i \in \mathscr{C})$ for the examples in Section 2.4. According to the table given in Section 2.4, for $\sigma_{0}, \sigma_{1}, \sigma_{2}, \sigma_{4}$ and $\sigma_{6}$ all conjugates of $\beta$ have the same modulus. Thus for these examples the box-counting dimension is equal to the Hausdorff dimension. Using Theorem 4.4 we calculate the following values.

- For $\sigma_{0}$ we have $\operatorname{dim}_{B}(\partial \mathcal{T})=\operatorname{dim}_{B}(\partial \mathcal{T}(i))=1.196510420 \ldots$

- For $\sigma_{1}$ we have $\operatorname{dim}_{B}(\partial \mathcal{T})=\operatorname{dim}_{B}(\partial \mathcal{T}(i))=1.100263385 \ldots$

- For $\sigma_{2}$ we have $\operatorname{dim}_{B}(\partial \mathcal{T})=\operatorname{dim}_{B}(\partial \mathcal{T}(i))=1.946434603 \ldots$

- For $\sigma_{3}$ we have $\operatorname{dim}_{B}(\partial \mathcal{T})=\operatorname{dim}_{B}(\partial \mathcal{T}(i))=1.630544213 \ldots$

- For $\sigma_{4}$ we have $\operatorname{dim}_{B}(\partial \mathcal{T})=\operatorname{dim}_{B}(\partial \mathcal{T}(i))=1.563995213 \ldots$

- For $\sigma_{5}$ we have $\operatorname{dim}_{B}(\partial \mathcal{T})=\operatorname{dim}_{B}(\partial \mathcal{T}(i))=1.744561766 \ldots$

- For $\sigma_{6}$ we have $\operatorname{dim}_{B}(\partial \mathcal{T})=\operatorname{dim}_{B}(\partial \mathcal{T}(i))=1.791903475 \ldots$

In the case of $\sigma_{0}$ the dimension calculations are detailed in Example 5.11. For all the other examples the calculations are similar to this case.

\subsection{Exclusive inner points and the geometric property (F)}

As mentioned in Definition 3.6, the well-known finiteness property ( $F$ ) for betaexpansions invented by Frougny and Solomyak [87] can be extended to DumontThomas numeration systems associated to a substitution and expressed in geometrical terms. In the context of beta-numeration, Akiyama [9] proved that property $(\mathrm{F})$ has consequences for the central tile $\mathcal{J}$. In particular, it is equivalent to the fact that $\mathbf{0}$ is an exclusive inner point of $\mathcal{T}=\mathcal{T}(1) \cup \cdots \cup \mathcal{T}(n)$. Notice that an exclusive point of a patch of finitely many tiles in a multiple tiling is a point that is contained exclusively in this patch and in no other tile of the multiple tiling.

We generalize Akiyama's result to arbitrary primitive unit Pisot substitutions by giving a geometric proof.

THEOREM 4.6. - Let $\sigma$ be a primitive unit Pisot substitution that satisfies the strong coincidence condition. Then $\sigma$ satisfies the geometric property $(F)$ (see Definition 3.6) if and only if $\mathbf{0}$ is an exclusive inner point of the patch $\mathcal{T}=\mathcal{T}(1) \cup \cdots \cup \mathcal{T}(n)$ in the self-replicating multiple tiling. If the geometric property $(F)$ holds, the self-replicating multiple tiling is a tiling.

Hence, the geometric property $(\mathrm{F})$ implies the tiling property, but the converse is not true (see Example 4.8).

The geometric property $(\mathrm{F})$ cannot be checked directly since it implies infinitely many iterations of $\mathbf{E}_{1}^{*}$ on $\mathcal{U}$. We provide an algorithm to check $(\mathrm{F})$ in terms of the zero-expansion graph, by considering the tiles containing $\mathbf{0}$ in the self-replicating multiple tiling.

THEOREM 4.7. - Let $\sigma$ be a primitive unit Pisot substitution over the alphabet $\mathscr{G}$ that satisfies the strong coincidence condition. Then $\sigma$ satisfies the geometric property $(F)$ if and only if the zero-expansion graph contains only nodes of the shape $[\mathbf{0}, i](i \in \mathscr{C})$. 
EXAMPLE 4.8. - For the substitutions defined in Section 2.4 we have the following situation.

- The substitutions $\sigma_{0}, \sigma_{1}$ and $\sigma_{4}$ satisfy the geometric property $(\mathrm{F})$.

- The substitutions $\sigma_{2}, \sigma_{3}, \sigma_{5}, \sigma_{6}$ do not satisfy the geometric property (F). For $\sigma_{3}$ for instance, $\mathbf{0}$ belongs to the four different tiles $\mathcal{T}(i)+\gamma$ with

$$
[\gamma, i] \in\{[\mathbf{0}, 2],[\mathbf{0}, 3],[\pi(1,1,0), 3],[\pi(-1,-1,1), 3]\} .
$$

As a second example we see that the substitution $\sigma_{5}$ does not satisfy the geometric property $(\mathrm{F})$ since $\mathbf{0} \in \mathcal{T}(i)+\gamma$ for $[\gamma, i] \in\{[\mathbf{0}, 1],[\mathbf{0}, 3],[\pi(1,-1,0), 1]$, $[\pi(-1,1,1), 2]\}$.

For $\sigma_{0}$ and $\sigma_{2}$ details are given in Example 5.3. The zero-expansion graph of $\sigma_{0}$ contains only one node with a loop, while the zero-expansion graph of $\sigma_{2}$ contains 8 nodes and is depicted in Figure 5.1. This means that $\mathbf{0}$ is contained in 8 subtiles of the self-replicating tiling associated to $\sigma_{2}$. Thus $\sigma_{2}$ does not satisfy the geometric property $(\mathrm{F})$ although it satisfies the tiling property. Thus the geometric property $(\mathrm{F})$ is strictly stronger than the tiling property.

\subsection{Connectivity properties of the central tile}

Apart from the Tribonacci substitution [141], sufficient conditions for connectivity appear in [59], but they are not algorithmic. In the following theorem we provide an effective way to check the connectivity of a central tile and its subtiles.

THEOREM 4.9. - Let $\sigma$ be a primitive unit Pisot substitution over the alphabet $\mathscr{G}$. Each subtile $\mathcal{T}(i) \quad(i \in \mathscr{C})$ is a locally connected continuum if and only if the tilerefinement-connectivity graph of $\mathcal{T}(j)$ is connected for each $j \in \mathscr{G}$.

The central tile $\mathcal{T}$ is a locally connected continuum if the tile-connectivity graph as well as each tile-refinement-connectivity graph is connected.

Notice that it is not easy to provide a necessary and sufficient condition for the connectivity of the central tile. The reason is that the central tile $\mathcal{T}$ might be connected even if some of the subtiles are disconnected. However, we were not able to find an example with this constellation.

ExAmPLE 4.10. - We will use Theorem 4.9 in Example 5.15 to prove that the central tile of $\sigma_{3}(1)=3, \sigma_{3}(2)=23, \sigma_{3}(3)=31223$ is not connected. Indeed the tileconnectivity graph is connected for this substitution, however, the tile-refinementconnectivity graph of $\mathcal{T}(2)$ is not connected (the tile is depicted in Figure 2.3).

For all the other substitutions given in Section 2.4 the central tiles as well as all their subtiles are connected. For $\sigma_{0}$ this is proved in Example 5.14. The proofs for all the other examples run along the same lines. 


\subsection{Disklikeness of the central tile and its subtiles}

Next we turn to the question whether a given tile $\mathcal{T}$ or one of its subtiles $\mathcal{T}(i)$ is homeomorphic to a closed disk. This implies that the dominant eigenvalue $\beta$ of the incidence matrix of $\sigma$ has degree 3 .

Examples of central tiles being homeomorphic to a closed disk are given in $[\mathbf{1 2 0}$, 127, 128], where a parametrization of the boundary is given for a class of substitutions related to the Tribonacci substitution. Here we start by proving the following criterion.

THEOREM 4.11. - Let $\sigma$ be a primitive unit Pisot substitution such that the dominant eigenvalue of its incidence matrix has degree 3. Suppose that the substitution satisfies the quotient mapping condition and that the lattice multiple tiling is a tiling.

Assume further that the associated central tile $\mathcal{T}$ is homeomorphic to a closed disk. Then there exist at most eight pairwise disjoint values $\gamma$ with $[\gamma, i] \in \Gamma_{\text {lat }}$ and

$$
\mathcal{J} \cap(\mathcal{J}+\gamma) \neq \varnothing .
$$

Among these eight values of $\gamma$ there are at most six ones for with the intersection in (4.4) contains more than one point.

These "neighbor constellations" of $\mathcal{T}$ can be checked algorithmically by checking the nodes of the lattice boundary graph of $\sigma$.

The previous result can often be used in order to prove that a given tile $\mathcal{T}$ is not homeomorphic to a disk by exhibiting too many "neighbors" $\mathcal{T}+\gamma$ of $\mathcal{T}$ in the induced lattice tiling.

EXAMPLE 4.12. - We discuss the theorem for two examples from Section 2.4.

The central tile of $\sigma_{4}(1)=11112, \sigma_{4}(2)=11113$ and $\sigma_{4}(3)=1$ is not homeomorphic to a disk (see the tile in its induced tilings in Figure 3.2). Indeed, in the lattice tiling the tile $\mathcal{T}$ has 10 neighbors (for details see Example 5.13 and the lattice boundary graph depicted in Figure 5.3).

Notice that the criterion is very precise: indeed, for the substitution $\sigma_{0}$, the (disklike) central tile $\mathcal{T}$ has eight neighbors, but four of the intersections are single points. Thus Theorem 4.11 cannot be used (see the tiling in Figure 3.3, the lattice boundary graph in Figure 5.4 and Example 5.13 for further details).

We will also prove the following criterion for the subtiles $\mathcal{T}(i)(i \in \mathscr{Q})$ to be homeomorphic to a closed disk.

THEOREM 4.13. - Let $\sigma$ be a primitive unit Pisot substitution such that the dominant eigenvalue of its incidence matrix has degree 3. Suppose that $\sigma$ has the tiling property.

Then each $\mathcal{T}(i)(i \in \mathscr{C})$ is homeomorphic to a closed disk if the following assertions hold.

1. Each boundary-connectivity graph is a simple loop.

2. Each boundary-refinement-connectivity graph is either empty or a single node or a line. 
3. Each intersection between three distinct tiles of the self-replicating tiling is either empty or a single point.

REMARK 4.14. - If a boundary-connectivity graph is a single node and the associated boundary-refinement-connectivity graph is a unique loop then the theorem is also true. The proof of this variant runs along the same lines as the proof of the theorem. As we could not construct a single example with this property we omit the details.

It is also possible to give a condition for the central tile $\mathcal{T}$ to be homeomorphic to a closed disk. This is the case for instance if all the subtiles $\mathcal{T}(i)(i \in \mathscr{G})$ are closed disks and if they intersect each other in a simple arc in a way that no holes occur. All this can be checked by means of our graphs.

EXAMPLE 4.15. - Each of the subtiles of the central tile of the substitutions $\sigma_{0}$ and $\sigma_{1}$ is homeomorphic to a closed disk. Details are given in Examples 5.34 and 5.35, respectively. All the other examples contained in Section 2.4 are not homeomorphic to a closed disk.

\subsection{The fundamental group of the central tile and its subtiles}

Finally, we are able to establish some results on the fundamental group of $\mathcal{T}(i)$ $(i \in \mathscr{G})$.

THEOREM 4.16. - Let $\sigma$ be a primitive unit Pisot substitution such that the dominant eigenvalue of its incidence matrix has degree 3. Suppose that $\sigma$ has the tiling property.

Then there exists a criterion which guarantees that each subtile $\mathcal{J}(i)(i \in \mathscr{C})$ have an uncountable fundamental group which is not free.

The detailed statement of this result is contained in Theorem 6.26 (see also Theorem 6.23 which contains a criterion for the fundamental group of $\mathcal{T}(i)(i \in \mathscr{G})$ to be nontrivial).

EXAmPLE 4.17. - The subtiles of the central tile of the substitution $\sigma_{5}(1)=123$, $\sigma_{5}(2)=1, \sigma_{5}(3)=31$ have an uncountable fundamental group which is not free. Details are given in Example 6.29.

The subtiles of the central tile for the flipped Tribonacci substitution $\sigma_{6}(1)=12$, $\sigma_{6}(2)=31, \sigma_{6}(3)=1$ have an uncountable fundamental group which is not free. Details are given in Example 6.28.

Also for $\sigma_{2}$ and $\sigma_{4}$ one can prove in a similar way as for $\sigma_{5}$ and $\sigma_{6}$ that the fundamental group of each of the subtiles of their central tiles has a fundamental group that is uncountable and not free. 


\section{CHAPTER 5}

\section{GRAPHS THAT CONTAIN TOPOLOGICAL INFORMATION ON THE CENTRAL TILE}

In this chapter we define the graphs that occur in our main results as well as their proofs. Indeed, these graphs are the main tools in order to describe topological properties of a central tile $\mathcal{T}$ and its subtiles $\mathscr{T}(i)(i \in \mathscr{C})$ by exploiting the set equation (2.7) as well as information on the intersections of tiles in the induced tilings. As will be seen throughout the chapter, many of these graphs are of interest in their own right.

In all what follows $\sigma$ is a primitive unit Pisot substitution over the alphabet $\mathscr{Q}$ with dominant eigenvalue $\beta$.

\subsection{The graph detecting expansions of zero}

Let us start our definitions by building a graph that permits to decide whether the origin $\mathbf{0}$ is contained in a given tile of the self-replicating tiling.

We provide the formal definition before we give some comments on it.

Definition 5.1 (Zero-expansion graph). - The zero-expansion graph $\mathscr{G}^{(\mathbf{0})}$ of $\sigma$ is the largest ${ }^{(1)}$ graph such that the following conditions hold.

1. The nodes $[\gamma, i]$ of the graph belong to the self-replicating translation set $\Gamma_{\mathrm{srs}}$ and satisfy

$$
\|\gamma\| \leq \frac{\max \{\|\pi \mathbf{l}(p)\| ;(p, a, s) \in \mathscr{P}\}}{1-\max \left\{\left|\beta^{(\ell)}\right| ; \ell=2, \ldots, d\right\}},
$$

where $\beta^{(\ell)}, \ell=2, \ldots, d$, denote the algebraic conjugates of $\beta$ (recall that $\|\cdot\|$ is the norm defined in (2.1)).

(1) By "largest" we mean that every set of nodes that satisfies the conditions has to be included in $\mathscr{G}^{(\mathbf{0})}$. We will prove that such a set exists. 
2. There is a directed edge from $\left[\gamma_{1}, i_{1}\right]$ to $\left[\gamma_{2}, i_{2}\right]$, if and only if there exists $\left(p_{1}, i_{1}, s_{1}\right) \in \mathscr{P}$ such that

$$
p_{1} i_{1} s_{1}=\sigma\left(i_{2}\right) \quad \text { and } \quad \mathbf{h} \gamma_{2}=\gamma_{1}+\pi \mathbf{l}\left(p_{1}\right) .
$$

3. Every node is the starting point of an infinite walk.

The zero-expansion graph is used to characterize all elements $[\gamma, i] \in \Gamma_{\text {srs }}$ for which the tile $\mathcal{T}(i)+\gamma$ contains $\mathbf{0}$. Suppose that $\mathbf{0} \in \mathcal{J}(i)+\gamma$. In view of Corollary 2.8, this implies that $\mathbf{0}=\gamma+\sum_{k \geq 0} \mathbf{h}^{k} \pi \mathbf{l}\left(p_{k}\right)$. In other words, in this case

$$
\|\gamma\| \leq \sum_{k \geq 0}\left\|\mathbf{h}^{k} \pi \mathbf{l}\left(p_{k}\right)\right\| \leq \frac{\max \{\|\pi \mathbf{l}(p)\| ;(p, a, s) \in \mathscr{P}\}}{1-\max \left\{\left|\beta^{(\ell)}\right| ; \ell=2, \ldots, d\right\}},
$$

where the second estimate follows from the fact that $\left\|\pi \mathbf{l}\left(p_{k}\right)\right\|$ is uniformly bounded and $\mathbf{h}$ is a uniform contraction with contraction ratio $\max \left\{\left|\beta^{(\ell)}\right| ; \ell=2, \ldots, d\right\}$. Thus, we can confine ourselves to elements $[\gamma, i] \in \Gamma_{\text {srs }}$ satisfying (5.1) when searching for tiles $\mathcal{T}(i)+\gamma$ containing $\mathbf{0}$. This justifies the bound (5.1) in Definition 5.1. As the edges of this graph are defined similar to the edges of the prefix-suffix-graph, it is not hard to see that infinite walks in this graphs correspond to $\mathbf{h}$-ary representations. The fact that this graph is suited for getting tiles containing the origin is contained in the following proposition.

Proposition 5.2. - The zero-expansion graph $\mathscr{G}^{(\mathbf{0})}$ of a primitive unit Pisot substitution is well defined and finite. A pair $[\gamma, i]$ is a node of this graph if and only if $\mathbf{0} \in \mathcal{J}(i)+\gamma$.

This type of graph first appeared in more specific settings in $[\mathbf{9}, \mathbf{1 4 1}, \mathbf{1 5 5}]$. For the sake of clarity, we detail the proof of Proposition 5.2 in Chapter 7.

Algorithmic construction. The zero-expansion graph $\mathscr{G}^{(\mathbf{0})}$ can be constructed algorithmically as follows.

(i) First we need to set up a finite set of candidates for the nodes of $\mathscr{G}^{(\mathbf{0})}$. By the definition of $\pi$, the first coordinate of each node $[\gamma, i]$ of $\mathscr{G}^{(\mathbf{0})}$ is of the form

$$
\gamma=\sum_{k=2}^{r+2 s}\left\langle\mathbf{x}, \mathbf{v}_{\beta^{(k)}}\right\rangle \mathbf{u}_{\beta^{(k)}},
$$

where $\mathbf{x} \in \mathbb{Z}^{n}$. Since $[\gamma, i]=[\pi(\mathbf{x}), i] \in \Gamma_{\text {srs }}$ we must have $0 \leq\left\langle\mathbf{x}, \mathbf{v}_{\beta}\right\rangle\left\langle\left\langle\mathbf{e}_{i}, \mathbf{v}_{\beta}\right\rangle\right.$. Moreover, by the definition of the norm $\|\cdot\|$ in (2.1), the bound in (5.1) implies that

$$
\left|\left\langle\mathbf{x}, \mathbf{v}_{\beta^{(k)}}\right\rangle\right| \leq \frac{\max \{\|\pi \mathbf{l}(p)\| ;(p, a, s) \in \mathscr{P}\}}{1-\max \left\{\left|\beta^{(\ell)}\right| ; \ell=2, \ldots, d\right\}} \quad(k \in\{2, \ldots, r+2 s\})
$$

has to hold. Thus $\left\langle\mathbf{x}, \mathbf{v}_{\beta}\right\rangle$ has to be a bounded element of $\mathbb{Z}[\beta]$ each of whose conjugate is bounded. Hence, there is only a finite number of elements $\gamma=$ $\pi(\mathbf{x}) \in \mathbb{H}_{c}$ with this property (the coefficients $n_{k} \in \mathbb{Z}$ of the relevant elements $\left\langle\mathbf{x}, \mathbf{v}_{\beta}\right\rangle=n_{0}+n_{1} \beta+\cdots+n_{d-1} \beta^{d-1}$ can be calculated by standard linear algebra; 


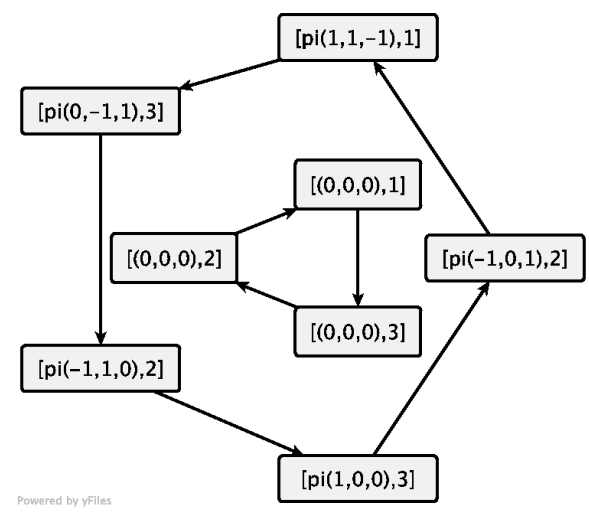

FiguRE 5.1. The zero-expansion graph for the substitution $\sigma_{2}(1)=2$, $\sigma_{2}(2)=3, \sigma_{2}(3)=12$. "pi" stands for the projection mapping $\pi$.

these coefficients are then used to calculate the coordinates of $\gamma$ in (5.3) by Galois conjugation). Denote the finite set of all $[\gamma, i]$ with $\gamma$ taken from this finite set by $V$.

(ii) For every element $\left[\gamma_{2}, i_{2}\right] \in \mathcal{V}$ we consider all decompositions $\sigma\left(i_{2}\right)=p_{1} i_{1} s_{1}$ and set $\gamma_{1}=\mathbf{h} \gamma_{2}-\pi \mathbf{l}\left(p_{1}\right)$. If $\left[\gamma_{1}, i_{1}\right] \in \mathcal{V}$ we draw an edge from $\left[\gamma_{1}, i_{1}\right]$ to $\left[\gamma_{2}, i_{2}\right]$.

(iii) To satisfy Condition 3 in the definition of the zero-expansion graph, we recursively remove all nodes that do not have outgoing edges. This implies that each of the remaining nodes is the starting point of an infinite walk.

EXAMPLE 5.3. - In this example we give the details for Example 4.8.

- The zero-expansion graph for the substitution $\sigma_{0}(1)=112, \sigma_{0}(2)=113, \sigma_{0}(3)=$ $4, \sigma_{0}(4)=1$ is equal to the node $[\mathbf{0}, 1]$ together with a self-loop. Thus the origin $\mathbf{0}$ only belongs to one subtile of the central tile in the self-replicating tiling. Hence, $\sigma_{0}$ satisfies the geometric property $(\mathrm{F})$.

- The zero-expansion graph for the substitution $\sigma_{2}(1)=2, \sigma_{2}(2)=3, \sigma_{2}(3)=12$ is shown in Figure 5.1. It consists of eight states which are grouped in two independent cycles. Hence, $\mathbf{0}$ belongs to eight tiles of the self-replicating tiling: it belongs to the three subtiles $\mathcal{T}(1), \mathcal{T}(2)$ and $\mathcal{T}(3)$ as well as to the translated subtiles $\mathcal{T}(1)+\pi(1,1,-1), \mathcal{J}(2)+\pi(-1,1,0), \mathcal{J}(2)+\pi(-1,0,1), \mathcal{T}(3)+\pi(1,0,0)$ and $\mathcal{T}(3)+\pi(0,-1,1)$. Thus $\sigma_{2}$ does not satisfy the geometric property $(\mathrm{F})$.

\subsection{Graphs describing intersections of two subtiles}

In this section our aim is to investigate the intersections between the subtiles of the central tile and some translated subtiles (corresponding to the self-replicating multiple tiling or the lattice multiple tiling). We introduce the following terminology. 
We will say that $\mathcal{T}(i)+\gamma_{1}$ and $\mathcal{T}(j)+\gamma_{2}$ with $\left[\gamma_{1}, i\right],\left[\gamma_{2}, j\right] \in \Gamma_{\text {srs }}$ are neighbors in the self-replicating multiple tiling if they have nonempty intersection. An analogous terminology is used for the tiles forming the lattice multiple tiling.

In this setting, the intersection $\mathcal{T}(i) \cap \mathcal{T}(j)+\gamma$ is described by the node $[i, \gamma, j] \in$ $\mathscr{C} \times \pi\left(\mathbb{Z}^{n}\right) \times \mathscr{C}$ of the so-called boundary graph. However, $[i, \gamma, j]$ and $[j,-\gamma, i]$ will then stand for the same intersection. To avoid this redundancy in the boundary graph, we reduce the set of possible nodes to

$$
\mathfrak{D}=\left\{[i, \gamma, j] \in \mathscr{G} \times \pi\left(\mathbb{Z}^{n}\right) \times \mathscr{G} ; \gamma=\pi(\mathbf{x}),\left(\left\langle\mathbf{x}, \mathbf{v}_{\beta}\right\rangle>0\right) \text { or }(\gamma=\mathbf{0} \text { and } i \leq j)\right\} .
$$

We are now going to define a class of graphs whose sets of nodes are finite subsets of $\mathfrak{D}$. After a formal definition of these graphs we will give some comments.

DEFinition 5.4 (Boundary graph). - Let $\& \subset \mathfrak{D}$ be a finite set. The boundary graph of $\phi$ is denoted by $\mathscr{G}^{(B)}(\phi)$. It is the largest ${ }^{(2)}$ graph such that the following conditions hold.

1. A triple $[i, \gamma, j]$ is a node of $\mathscr{G}^{(B)}(\varnothing)$ if $[i, \gamma, j] \in \mathfrak{D}$ and

$$
\|\gamma\| \leq \frac{2 \max \{\|\pi \mathbf{l}(p)\| ;(p, a, s) \in \mathscr{P}\}}{1-\max \left\{\left|\beta^{(\ell)}\right| ; \ell=2, \ldots, d\right\}}
$$

where $\beta^{(\ell)}$ are the algebraic conjugates of $\beta$.

2. There is a directed edge from $[i, \gamma, j]$ to $\left[i^{\prime}, \gamma^{\prime}, j^{\prime}\right]$, if and only if there exist $[\bar{i}, \bar{\gamma}, \bar{j}] \in \mathscr{G} \times \pi\left(\mathbb{Z}^{n}\right) \times \mathscr{G}$ and $\left[\left(p_{1}, a_{1}, s_{1}\right),\left(p_{2}, a_{2}, s_{2}\right)\right] \in \mathscr{P} \times \mathscr{P}$ such that

$$
\left\{\begin{array}{l}
\left.\left[i^{\prime}, \gamma^{\prime}, j^{\prime}\right]=[\bar{i}, \bar{\gamma}, \bar{j}](\text { type } 1) \text { or }\left[i^{\prime}, \gamma^{\prime}, j^{\prime}\right]=[\bar{j},-\bar{\gamma}, \bar{i}] \text { (type } 2\right), \\
a_{1}=i \text { and } p_{1} a_{1} s_{1}=\sigma(\bar{i}) \\
a_{2}=j \text { and } p_{2} a_{2} s_{2}=\sigma(\bar{j}) \\
\mathbf{h} \bar{\gamma}=\gamma+\pi\left(\mathbf{l}\left(p_{2}\right)-\mathbf{l}\left(p_{1}\right)\right)
\end{array}\right.
$$

The edge is labeled by

$$
\eta= \begin{cases}\pi \mathbf{l}\left(p_{1}\right), & \left\langle\mathbf{l}\left(p_{1}\right), \mathbf{v}_{\beta}\right\rangle \leq\left\langle\mathbf{l}\left(p_{2}\right)+\mathbf{x}, \mathbf{v}_{\beta}\right\rangle, \\ \pi \mathbf{l}\left(p_{2}\right)+\gamma, & \text { otherwise, }\end{cases}
$$

where $\mathbf{x} \in \mathbb{Z}^{n}$ is chosen in a way that $\pi(\mathbf{x})=\gamma$.

3. Each node belongs to an infinite walk starting from a node $[i, \gamma, j] \in \varnothing$.

First we explain why we use the bound (5.4). Note that we want to describe intersections of the shape $\mathcal{J}(i) \cap \mathcal{J}(j)+\gamma$. In view of Corollary 2.8 these two subtiles intersect if and only if the equation

$$
\sum_{k \geq 0} \mathbf{h}^{k} \pi \mathbf{l}\left(p_{1}^{(k)}\right)=\gamma+\sum_{k \geq 0} \mathbf{h}^{k} \pi \mathbf{l}\left(p_{2}^{(k)}\right)
$$

(2) The meaning of "largest" is explained in Definition 5.1. 
has a solution for two walks in the prefix-suffix graph labeled by $\left(p_{1}^{(k)}, i^{(k)}, s_{1}^{(k)}\right)$ and $\left(p_{2}^{(k)}, j^{(k)}, s_{2}^{(k)}\right)$, respectively (here $i=i^{(0)}$ and $\left.j=j^{(0)}\right)$. However, this equation can hold only if

$$
\|\gamma\| \leq \sum_{k \geq 0}\left\|\mathbf{h}^{k} \pi \mathbf{l}\left(p_{1}^{(k)}\right)\right\|+\sum_{k \geq 0}\left\|\mathbf{h}^{k} \pi \mathbf{l}\left(p_{2}^{(k)}\right)\right\| \leq \frac{2 \max \{\|\pi \mathbf{l}(p)\| ;(p, a, s) \in \mathscr{P}\}}{1-\max \left\{\left|\beta^{(\ell)}\right| ; \ell=2, \ldots, d\right\}}
$$

where the estimates follow as in (5.2).

The definition of the edges in this graph is directly deduced from (4.1). Note that each point in the intersection $\mathcal{T}(i) \cap(\mathcal{J}(j)+\gamma)$ has two h-ary representations. One with "integer part" $\mathbf{0}$ and one with "integer part" $\gamma$. Indeed, the sequence of edges in the boundary graph starting from a node $[i, \gamma, j]$ produces two walks $\left(p_{1}^{(k)}, i^{(k)}, s_{1}^{(k)}\right)$ and $\left(p_{2}^{(k)}, j^{(k)}, s_{2}^{(k)}\right)$ (the distinction between walks of type 1 and 2 has technical reasons that will become apparent in the proofs). These two walks correspond to these two h-ary representations.

Many of the graphs defined in the sequel are defined by these ideas. These are the contact graph (Definition 5.19), the triple point graph (Definition 5.24) and the quadruple point graph (Definition 7.2).

Proposition 5.5. - The boundary graph associated with a finite subset $\&$ of $\mathfrak{D}$ is well defined and finite.

We give the full proof of this proposition in Chapter 7. It implies that a boundary graph can be algorithmically computed as soon as the set $\phi$ is known. Indeed, start with the set of nodes $\phi$. Then recursively increase this set with nodes $\left[i^{\prime}, \gamma^{\prime}, j^{\prime}\right]$ that satisfy conditions (1) and (2). The finiteness property in Proposition 5.5 ensures that this procedure will eventually stabilize the set of nodes. From this final set, nodes with no outgoing edges have to be removed recursively, so that condition (3) is also fulfilled.

TheOREM 5.6. - Let $\&$ be a finite subset of $\mathfrak{D}$. Let $[i, \gamma, j] \in \varnothing$. The intersection between $\mathcal{T}(i)$ and $\mathcal{T}(j)+\gamma$ is nonempty if and only if $[i, \gamma, j]$ is a node of the boundary graph $\mathscr{G}^{(B)}(\phi)$.

The proof is given in Chapter 7.

Depending on the set $\phi$, we build several boundary graphs that have particular relevance. 
5.2.1. The self-replicating boundary graph. - The self-replicating tiling is based on the set $\Gamma_{\text {srs }}$ (see Definition 3.2) that is the set of pairs $[\pi(\mathbf{x}), i]$ satisfying the condition $0 \leq\left\langle\mathbf{x}, \mathbf{v}_{\beta}\right\rangle\left\langle\left\langle\mathbf{e}_{j}, \mathbf{v}_{\beta}\right\rangle\right.$. Hence, the set of points in $\Gamma_{\text {srs }}$ that fulfill condition (5.4) is finite (the proof is similar to the proof of Proposition 5.5). This implies that also the set

$$
\phi_{\text {srs }}:=\left\{\begin{array}{l|l}
{[i, \gamma, j] \in \mathfrak{D} ;} & \begin{array}{l}
\gamma=\pi(\mathbf{x}), \mathbf{x} \in \mathbb{Z}^{n}, 0 \leq\left\langle\mathbf{x}, \mathbf{v}_{\beta}\right\rangle<\left\langle\mathbf{e}_{j}, \mathbf{v}_{\beta}\right\rangle \\
\gamma \neq \mathbf{0} \text { or } i \neq j \\
\gamma \text { satisfies condition (5.4) }
\end{array}
\end{array}\right\} .
$$

is a finite set. The self-replicating boundary graph (SR-boundary graph, for short) is the boundary graph of this set, i.e.,

$$
\mathscr{G}_{\mathrm{srs}}^{(B)}=\mathscr{G}^{(B)}\left(\phi_{\mathrm{srs}}\right) .
$$

By definition, this graph informs about tiles of the self-replicating tiling that intersect the central tile.

Algorithmic construction. In view of the remarks after Proposition 5.5 it remains to construct the set $\phi_{\text {srs }}$. However, similar as in the algorithmic construction of the zero-expansion graph we see that $[i, \gamma, j] \in \phi_{\text {srs }}$ implies that $\gamma=\sum_{k=2}^{r+2 s}\left\langle\mathbf{x}, \mathbf{v}_{\beta^{(k)}}\right\rangle \mathbf{u}_{\beta^{(k)}}$, where $\left\langle\mathbf{x}, \mathbf{v}_{\beta}\right\rangle$ is an element of $\mathbb{Z}[\beta]$ with uniformly bounded Galois conjugates. Just use the bound (5.4) instead of (5.1). Thus there are finitely many candidates $\gamma$ for $[i, \gamma, j] \in \phi_{\text {srs }}$. It is now easy to identify the elements of $\phi_{\text {srs }}$ among these candidates by checking the conditions of its definition in (5.6).

THEOREM 5.7. - Let $\sigma$ be a primitive unit Pisot substitution and let $[i, \gamma, j]$ be a node of the SR-boundary graph $\mathscr{G}_{\mathrm{srs}}^{(B)}$. Let $B[i, \gamma, j]$ denote the nonempty compact set that is uniquely defined by the GIFS

$$
B[i, \gamma, j]=\underset{[i, \gamma, j] \stackrel{\eta}{\rightarrow}\left[i_{1}, \gamma_{1}, j_{1}\right] \text { in } \varphi_{\mathrm{srs}}^{(B)}}{\bigcup} \mathbf{h} B\left[i_{1}, \gamma_{1}, j_{1}\right]+\eta
$$

which is directed by the graph $\mathscr{G}_{\mathrm{srs}}^{(B)}$.

Then the following assertions are true.

- If $[i, \gamma, j]$ is a node of the SR-boundary graph $\mathscr{G}_{\mathrm{srs}}^{(B)}$, then $[\gamma, j]$ belongs to the selfreplicating translation set $\Gamma_{\mathrm{srs}}$ so that $\mathcal{T}(j)+\gamma$ is a piece of the self-replicating multiple tiling.

- The intersections between two tiles are the solution of (5.7). In particular, the solution $B[i, \gamma, j]$ of this GIFS equation satisfies ${ }^{(3)}$

$$
B[i, \gamma, j]=\mathcal{J}(i) \cap(\mathcal{T}(j)+\gamma) .
$$

(3) This justifies the notation $B[i, \gamma, j]$ in (5.7). 
- If the self-replicating multiple tiling is a tiling, then the boundary of each subtile of the central tile is described by the sets $B[i, \gamma, j]$, in particular,

$$
\partial \mathcal{T}(i):=\bigcup_{[\gamma, j] \in \Gamma_{\mathrm{srs}}:[i, \gamma, j] \in \mathscr{G}_{\mathrm{srs}}^{(B)}} B[i, \gamma, j] \quad(i \in \mathscr{G})
$$

(here we identify $[i, 0, j]$ with $[j, 0, i]$ in order to capture also the intersections $\mathcal{J}(i) \cap \mathcal{T}(j)$ with $j<i)$.

The proof of this theorem is given in Chapter 7. We also mention that this result is used to prove the first part of Theorem 4.1.

LEMMA 5.8. - Assume that $\sigma$ has the tiling property. If $B[i, \gamma, j]$ contains only one point it can be omitted in the representation of $\partial \mathcal{T}$ in (5.8).

Proof. - Suppose that $B[i, \gamma, j]$ contains a single point. If this single point is not isolated in $\partial \mathcal{T}(i)$ then $B[i, \gamma, j]$ has to be contained in another intersection $B\left[i^{\prime}, \gamma^{\prime}, j^{\prime}\right]$ because each of these intersections is compact. So let us assume that $B[i, \gamma, j]$ is an isolated point of $\partial \mathcal{T}(i)$. Then, as $\mathcal{T}(i)$ is the closure of its interior, each point in a small neighborhood of $B[i, \gamma, j]$ belongs to int $(\mathcal{T}(i)$ ) (note that each two points in this neighborhood are not separated by $\partial \mathcal{T}(i)$ ). Thus the single point contained in the intersection $B[i, \gamma, j]$ is an inner point of $\mathcal{T}(i)$, a contradiction.

From the GIFS equation (5.7) we deduce that each point in $\mathcal{T}(i) \cap(\mathcal{T}(j)+\gamma)$ can be expanded w.r.t. $\mathbf{h}$ by following the edge labels of infinite walks in the SR-boundary graph.

Corollary 5.9. - Let $[i, \gamma, j] \in \mathfrak{D}$. A point $\mathbf{x}$ belongs to the intersection $\mathcal{T}(i) \cap$ $(\mathcal{T}(j)+\gamma)$ if and only if there exists an infinite walk in $\mathscr{G}_{\mathrm{srs}}^{(B)}$, starting from $[i, \gamma, j]$ and labelled by $\left(\eta^{(k)}\right)_{k \geq 0}$ such that

$$
\mathbf{x}=\sum_{k \geq 0} \mathbf{h}^{k} \eta^{(k)}
$$

This is an immediate consequence of (5.7) (it is proved analogously to Corollary 2.8).

Note that Theorem 5.7 and Corollary 5.9 can be used to draw boundaries of central tiles and their subtiles. They were used to draw the boundaries of the tiles in the figures of the present monograph.

We need the following definition.

Definition 5.10. - Two infinite walks in a boundary graph $\mathscr{G}^{(B)}$ are called essentially different if their labellings $\left(\eta_{1}^{(k)}\right)_{k \geq 0}$ and $\left(\eta_{2}^{(k)}\right)_{k \geq 0}$ give rise to two different expansions, i.e., if

$$
\sum_{k \geq 0} \mathbf{h}^{k} \eta_{1}^{(k)} \neq \sum_{k \geq 0} \mathbf{h}^{k} \eta_{2}^{(k)}
$$




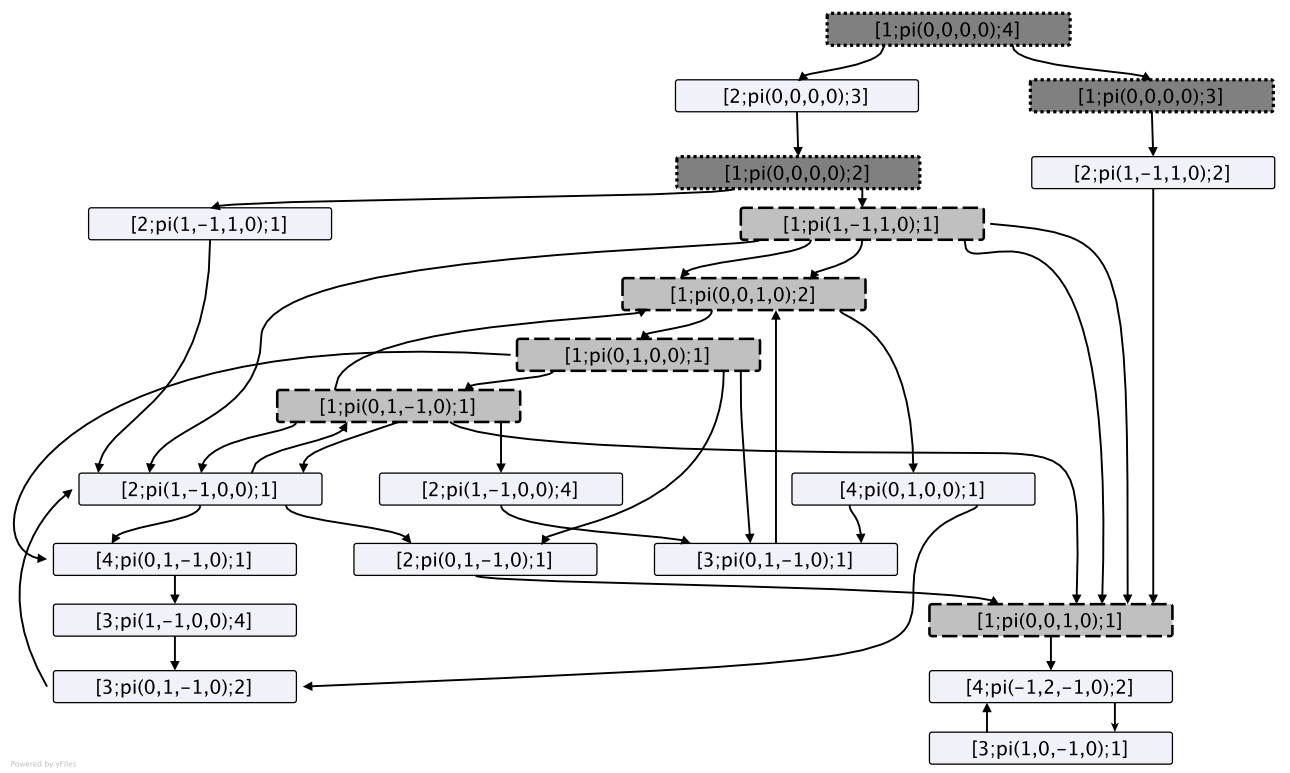

FiguRE 5.2. The SR-boundary graph for the substitution $\sigma_{0}(1)=112$, $\sigma_{0}(2)=113, \sigma_{0}(3)=4, \sigma_{0}(4)=1$. Labels of edges are omitted, "pi" stands for the projection mapping $\pi$. A node $[i, \gamma, j]$ occurs in this graph if and only if $\mathcal{T}(i)$ intersects the tile $\mathcal{T}(j)+\gamma$ in the self-replicating tiling.

For instance, the tile $\mathcal{T}(1)$ intersects the three other subtiles $\mathcal{T}(2), \mathcal{T}(3)$ and $\mathscr{T}(4)$ since the nodes $[1, \mathbf{0}, 2],[1, \mathbf{0}, 3]$ and $[1, \mathbf{0}, 4]$ appear in the graph (nodes with dotted frame). The tile $\mathcal{T}(1)$ also intersects five tiles outside the central tile, namely $\mathcal{T}(1)+\pi(0,0,1,0), \mathcal{T}(2)+\pi(0,0,1,0)$, $\mathcal{T}(1)+\pi(0,1,-1,0), \mathcal{T}(1)+\pi(0,1,0,0)$ and $\mathcal{T}(1)+\pi(1,-1,1,0)$, since the corresponding nodes appear in the graph. These are the nodes $[1, \pi(0,0,1,0), 1], \quad[1, \pi(0,0,1,0), 2],[1, \pi(0,1,-1,0), 1], \quad[1, \pi(0,1,0,0), 1]$, $[1, \pi(1,-1,1,0), 1]$ (nodes with dashed frame).

As we shall see later ( $c f$. Proposition 5.33) we can check algorithmically whether a given node of the SR-boundary graph is the starting point of one or more essentially different walks. This is equivalent to the fact that the associated intersection contains one or more points.

ExAmPLE 5.11. - The SR-boundary graph for $\sigma_{0}(1)=112, \sigma_{0}(2)=113, \sigma_{0}(3)=4$, $\sigma_{0}(4)=1$ is depicted in Figure 5.2. As we know the SR-boundary graph of $\sigma_{0}$ we can easily calculate the dominant eigenvalue $\mu=1.839286755 \ldots$ of its incidence matrix. Since $\mu$ is strictly less than the dominant eigenvalue $\beta=2.769292354 \ldots$ of the incidence matrix of $\sigma_{0}$, Theorem 4.1 shows that the self-replicating multiple tiling as well as the lattice tiling induced by $\sigma_{0}$ are actually tilings. Moreover, the knowledge of $\mu, \beta$ and the modulus of the smallest eigenvalue of the incidence matrix 
of $\sigma_{0}$ which is given by $\left|\beta^{\prime}\right|=0.6009185307 \ldots$ yields in view of Theorem 4.4 that

$$
\operatorname{dim}_{B}(\partial \mathcal{T})=\operatorname{dim}_{B}(\partial \mathcal{T}(i))=1.196510420 \ldots
$$

Since, according to the table given in Section 2.4, the conjugates of $\beta$ have all the same modulus the Hausdorff dimension of the boundaries $\partial \mathcal{T}(i)$ is the same as their box-counting dimension in this case.

We also see from the SR-boundary graph that the subtiles $\mathcal{T}(1), \mathcal{J}(2), \mathcal{J}(3), \mathcal{J}(4)$ of the central tile $\mathcal{T}$ intersect with 11 tiles in the self-replicating tiling, corresponding to pairs $[\gamma, j] \in \Gamma_{\text {srs }}$ with $\gamma \neq \mathbf{0}$. Indeed, these intersections appear as nodes $[i, \gamma, j]$ with $\gamma \neq \mathbf{0}$ in the SR-boundary graph.

Since $\sigma_{0}$ satisfies the tiling property, the SR-boundary graph can be used to describe the boundary of $\mathcal{T}$ and its subtiles. For example, as mentioned in the caption of Figure 5.2 the boundary of $\mathcal{J}(1)$ consists of eight intersections: three intersections with tiles inside the central tile $\mathcal{T}$ and five intersections with tiles outside the central tile. In particular,

$$
\begin{aligned}
\partial \mathcal{T}(1)= & B[1, \mathbf{0}, 2] \cup B[1, \mathbf{0}, 3] \cup B[1, \mathbf{0}, 4] \cup B[1, \pi(0,0,1,0), 1] \\
& \cup B[1, \pi(0,0,1,0), 2] \cup B[1, \pi(0,1,-1,0), 1] \cup B[1, \pi(0,1,0,0), 1] \\
& \cup B[1, \pi(1,-1,1,0), 1] .
\end{aligned}
$$

Note that in the boundary graph a unique infinite walk goes out from each of the nodes $[1, \mathbf{0}, 3]$ and $[1, \pi(0,0,1,0), 1]$. This means that each of the pieces $\mathcal{J}(1) \cap \mathcal{J}(3)$ and $\mathcal{T}(1) \cap(\mathcal{J}(1)+\pi(0,0,1,0))$ consists of a single point. Therefore by Lemma 5.8 these pieces can be omitted in the description of the boundary of $\mathcal{T}(1)$. Thus

$$
\begin{aligned}
\partial \mathcal{T}(1)= & B[1, \mathbf{0}, 2] \cup B[1, \mathbf{0}, 4] \cup B[1, \pi(0,0,1,0), 2] \\
& \cup B[1, \pi(0,1,-1,0), 1] \cup B[1, \pi(0,1,0,0), 1] \cup B[1, \pi(1,-1,1,0), 1] .
\end{aligned}
$$

5.2.2. The lattice boundary graph. - Here we suppose that $\sigma$ satisfies the quotient mapping condition (see Definition 3.14). Then the points of the lattice translation set that satisfy condition (5.4) is finite. The lattice boundary graph is the boundary graph of this set, i.e.,

$$
\mathscr{G}_{\text {lat }}^{(B)}=\mathscr{G}^{(B)}\left(\phi_{\text {lat }}\right)
$$

with

$$
\phi_{\text {lat }}:=\left\{\begin{array}{l|l}
{[i, \gamma, j] \in \mathfrak{D} ;} & \begin{array}{l}
{[\gamma, j] \in \Gamma_{\text {lat }} \backslash\{[\mathbf{0}, i]\}} \\
\gamma \text { satisfies condition (5.4) }
\end{array}
\end{array}\right\} .
$$

Algorithmic construction. Again by the considerations after Proposition 5.5 it is sufficient to construct the set $\phi_{\text {lat }}$. Let $[i, \gamma, j] \in \phi_{\text {lat }}$. Then there exist $n_{2}, \ldots, n_{d} \in$ $\mathbb{Z}$ such that $\gamma=\sum_{k=2}^{d} n_{i}\left(\pi\left(\mathbf{e}_{B(k)}\right)-\pi\left(\mathbf{e}_{B(1)}\right)\right)$. This implies that $\gamma \in \pi\left(\mathbb{Z}^{n}\right)$. In particular, we will choose

$$
\mathbf{x}=\sum_{k=2}^{d} n_{i}\left(\mathbf{e}_{B(k)}-\mathbf{e}_{B(1)}\right)
$$


Using this element $\mathbf{x}$ we get the representation

$$
\gamma=\sum_{k=2}^{d}\left\langle\mathbf{x}, \mathbf{v}_{\beta^{(k)}}\right\rangle \mathbf{u}_{\beta^{(k)}} .
$$

Thus, according to (5.9), to determine $\phi_{\text {lat }}$ we have to find all $\mathbf{x} \in \mathbb{Z}^{n}$ satisfying

$$
\begin{aligned}
\left|\left\langle\mathbf{x}, \mathbf{v}_{\beta^{(k)}}\right\rangle\right| & <\frac{2 \max \{\|\pi \mathbf{l}(p)\| ;(p, a, s) \in \mathscr{P}\}}{1-\max \left\{\left|\beta^{(\ell)}\right| ; \ell=2, \ldots, d\right\}} \quad(k \in\{2, \ldots, d\}), \\
\left\langle\mathbf{x}, \mathbf{e}_{k}\right\rangle & =0 \quad(k \in\{1, \ldots, n\} \backslash\{B(1), \ldots, B(d)\}),
\end{aligned}
$$

$\left\langle\mathbf{x}, \mathbf{e}_{B(1)}+\mathbf{e}_{B(2)}+\cdots+\mathbf{e}_{B(d)}\right\rangle=0$.

The first set of restriction is due to (5.4), the second and third one comes from the fact that $\gamma=\pi(\mathbf{x}) \in \Gamma_{\text {lat }}$ with $\mathbf{x}$ chosen as in (5.10). Together, these $n$ restrictions yield uniform bounds of the scalar products $\langle\mathbf{x}, \mathbf{e}\rangle$ for $n$ linearly independent vectors $\mathbf{e} \in \mathbb{R}^{n}$. As $\mathbb{Z}^{n}$ is a lattice there exist finitely many $\mathbf{x}$ satisfying these bounds. The set of these values $\mathbf{x}$ can thus be algorithmically constructed by checking (5.11).

As in Theorem 5.7, by its definition the lattice boundary graph informs about tiles of the lattice tiling that intersect the central tile. In particular, we have the following result.

Proposition 5.12. - Assume that $\sigma$ satisfies the quotient mapping condition. Let $[\gamma, j] \in \Gamma_{\text {lat }} \backslash\{[\mathbf{0}, i]\}$. The intersection between the subtile $\mathcal{T}(i)$ and the subtile $\mathcal{T}(j)+\gamma$ in the lattice tiling is nonempty if and only if $[i, \gamma, j]$ or $[j,-\gamma, i]$ is a node of the lattice boundary graph.

The lattice boundary graph is used in Theorem 4.11 to obtain a sufficient condition for non-dislikeness of $\mathcal{J}$. Contrarily to the set $\Gamma_{\text {srs }}$ in the SR-boundary graph, the lattice translation set $\Gamma_{\text {lat }}$ is not stable by the edge condition (5.5). Thus there may well exist nodes in the lattice boundary graph that correspond to elements not belonging to $\Gamma_{\text {lat }}$.

EXAMPLE 5.13. - In Figure 5.3 the lattice boundary graph of $\sigma_{4}$ is depicted. From this figure we deduce that $\mathcal{T} \cap(\mathcal{T}+\gamma) \neq \varnothing$ if and only if $\gamma \in \pm\{\pi(1,-1,0), \pi(0,1,-1)$, $\pi(1,0,-1), \pi(0,2,-2), \pi(1,1,-2)\}$. Thus the central tile has 10 neighbors and, hence, Theorem 4.11 applies to deduce that the central tile associated to $\sigma_{4}$ is not homeomorphic to a closed disk (the tile together with its neighbors in the induced tilings is depicted in Figure 3.2).

In Figure 5.4 the lattice boundary graph of $\sigma_{0}$ is depicted. From this figure we deduce that $\mathcal{T} \cap(\mathcal{T}+\gamma) \neq \varnothing$ if and only if $\gamma \in \pm\{\pi(1,-1,0,0), \pi(1,0,-1,0)$, $\pi(1,0,-1,0), \pi(1,-2,1,0)\}$. Thus $\mathcal{T}$ has eight neighbors. Notice that a unique infinite walk starts from the node $[3 ; \pi(1,0,-1,0) ; 1]$ and from the node $[2 ; \pi(1,-2,1,0) ; 4]$. Moreover, these nodes are the only nodes in the graph containing the vector $\pi(1,0,-1,0)$ and $\pi(1,-2,1,0)$ in their second coordinate, respectively. This implies that each of the intersections $\mathcal{T} \cap(\mathcal{T} \pm \pi(1,0,-1,0))$ and $\mathcal{T} \cap(\mathcal{T} \pm \pi(1,-2,1,0))$ is equal to a single point. Thus Theorem 4.11 cannot be applied. 


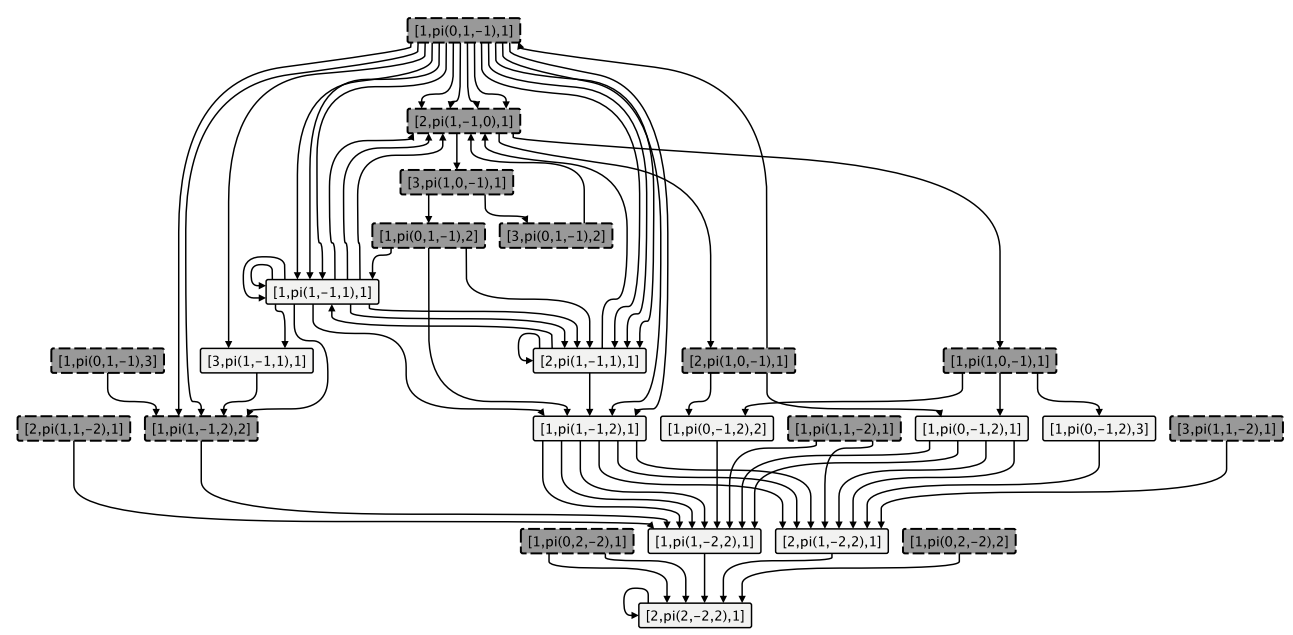

FiguRE 5.3. The lattice boundary graph for the substitution $\sigma_{4}(1)=$ $11112, \sigma_{4}(2)=11113, \sigma_{4}(3)=1$. Nodes with dashed frame correspond to intersections in the lattice tiling, i.e., nodes $[i, \gamma, j]$ with $[\gamma, j] \in \Gamma_{\text {lat }}$.

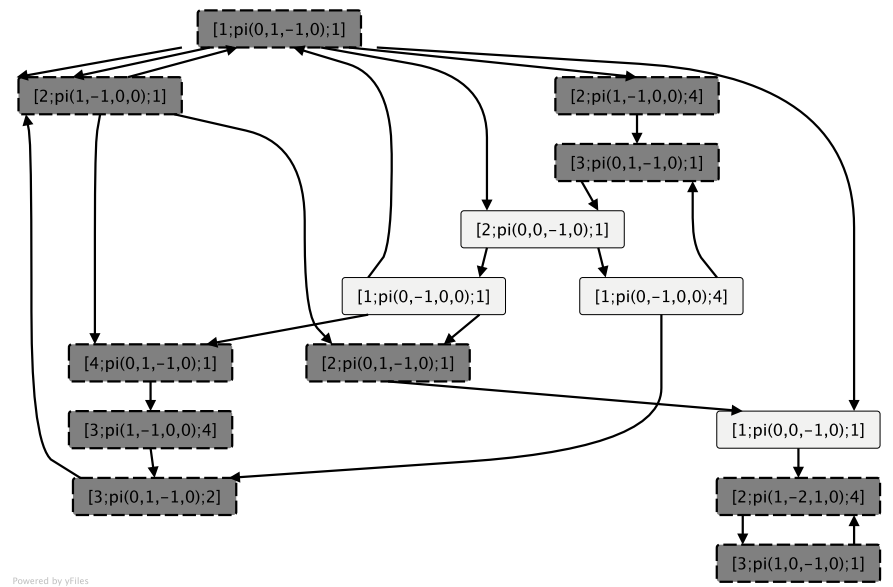

FiguRE 5.4. The lattice boundary graph for the substitution $\sigma_{0}(1)=112$, $\sigma_{0}(2)=113, \sigma_{0}(3)=4, \sigma_{0}(4)=1$. Nodes with dashed frame correspond to intersections in the lattice tiling. These are the nodes $[i, \gamma, j]$ with $[\gamma, j] \in \Gamma_{\text {lat }}$.

\subsection{Graphs related to the connectivity of the central tile}

The following graphs make it possible to decide whether certain sets are connected or not. 

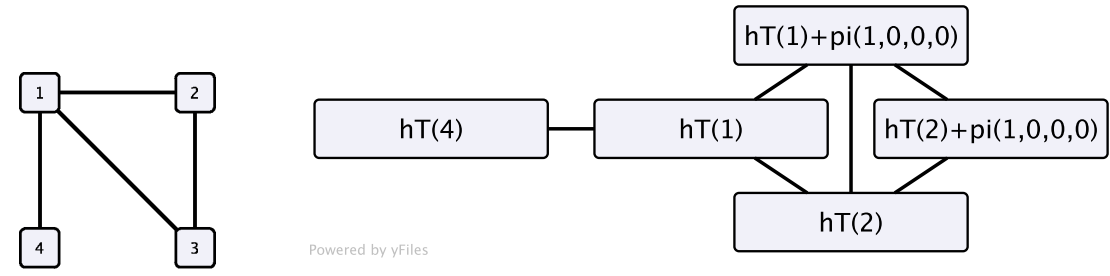

Figure 5.5. The tile-connectivity graph (left side) and the tile-refinementconnectivity graph of $\mathcal{T}(1)$ (right side) for the substitution $\sigma_{0}$. The notation "pi" stands for $\pi$ in the labelling. Additionally, we check that for each of the subtiles $\mathcal{T}(2), \mathcal{J}(3)$ and $\mathcal{T}(4)$ the tile-refinement-connectivity graph is equal to a single node. Therefore, all the tile-refinement-connectivity graphs and the tile-connectivity graph are connected. This implies that the central tile is connected (see Figure 2.1 for a picture of the tile).

Tile-connectivity graph : This undirected graph encodes intersections between tiles in the central tile. Its nodes are the elements of $\mathscr{G}$. There is an edge from $i$ to $j$ if and only if $\mathcal{T}(i)$ and $\mathcal{T}(j)$ intersect.

Concretely, there is an edge from $i$ to $j$ if $[i, \mathbf{0}, j]$ or $[j, \mathbf{0}, i]$ belongs to the SRboundary graph.

Tile-refinement-connectivity graph : According to Theorem 2.6, the tiles $\mathcal{J}(i)(i \in$ $\mathscr{G})$ are the solution of a GIFS governed by the prefix-suffix graph. Thus each $\mathcal{J}(i)$ can be represented as

$$
\mathcal{T}(i)=\bigcup_{i \stackrel{(p, i, s)}{\longrightarrow} j} \mathbf{h} \mathcal{T}(j)+\pi \mathbf{l}(p) .
$$

Denote the sets in the union on the right hand side by $T_{i 1}, \ldots, T_{i \ell}$. These sets are the nodes of the tile-refinement-connectivity graph of $\mathcal{T}(i)$. Each two of them are connected by an edge if and only if they have a nonempty intersection. Therefore, there is an edge between $\mathbf{h} \mathcal{J}\left(j_{1}\right)+\pi \mathbf{l}\left(p_{1}\right)$ and $\mathbf{h} \mathcal{T}\left(j_{2}\right)+\pi \mathbf{l}\left(p_{2}\right)$ if and only if $\left[j_{1}, \mathbf{h}^{-1} \pi\left(\mathbf{l}\left(p_{2}\right)-\mathbf{l}\left(p_{1}\right)\right), j_{2}\right]$ or $\left[j_{2}, \mathbf{h}^{-1} \pi\left(\mathbf{l}\left(p_{1}\right)-\mathbf{l}\left(p_{2}\right)\right), j_{1}\right]$ is a node of the SR-boundary graph.

EXAMPLE 5.14. - The central tile of $\sigma_{0}$ is depicted in Figure 2.1 together with its GIFS decomposition. We now prove that this tile as well as each of its subtiles is connected.

We first use the SR-boundary graph of $\sigma_{0}$ (see Figure 5.2) to build the tileconnectivity graph. We check that $[1, \mathbf{0}, 2],[1, \mathbf{0}, 3],[1, \mathbf{0}, 4],[2, \mathbf{0}, 3]$ are nodes of the SR-boundary graph. Thus we obtain the tile-connectivity graph depicted in Figure 5.5. It means that the largest tile $\mathcal{T}(1)$ intersects all the tiles, while the second 
and third tiles $\mathcal{J}(2)$ and $\mathcal{T}(3)$ intersect each other and the smallest tile $\mathcal{T}(4)$ only intersect the largest one $\mathcal{T}(1)$. Therefore the tile-connectivity graph is connected.

The tile-refinement-connectivity graphs for $\mathcal{T}(2), \mathcal{T}(3)$ and $\mathcal{T}(4)$ are equal to a single node since only one subset appears in their GIFS decomposition (see details in Section 2.4). The last graph to compute is the tile-refinement-connectivity graph of $\mathcal{J}(1)$ : it has five nodes, corresponding to the tiles that appear in the decomposition of $\mathcal{T}(1)$, namely $\mathbf{h} \mathcal{T}(1), \mathbf{h} \mathcal{T}(1)+\pi(1,0,0,0), \mathbf{h} \mathcal{J}(2), \mathbf{h} \mathcal{J}(2)+\pi(1,0,0,0), \mathbf{h} \mathcal{T}(4)$.

We use the SR-boundary graph to check intersections between these tiles, that is, to check whether the SR-boundary graph contains the nodes $\left[1, \mathbf{h}^{-1} \pi(1,0,0,0), 1\right]$, $[1, \mathbf{0}, 2], \quad[1, \mathbf{0}, 4], \quad\left[1, \mathbf{h}^{-1} \pi(1,0,0,0), 2\right], \quad\left[2, \mathbf{h}^{-1} \pi(1,0,0,0), 1\right], \quad\left[2, \mathbf{h}^{-1} \pi(1,0,0,0), 2\right]$, $[2, \mathbf{0}, 4],\left[4, \mathbf{h}^{-1} \pi(1,0,0,0), 1\right],\left[4, \mathbf{h}^{-1} \pi(1,0,0,0), 2\right]$, or their symmetric nodes. We deduce that the tile-refinement-connectivity graph of $\mathcal{T}(1)$ is connected (it is depicted in Figure 5.5).

By Theorem 4.9, we conclude that all the tiles $\mathcal{J}(1), \mathcal{T}(2), \mathcal{T}(3), \mathcal{T}(4)$ as well as the central tile $\mathcal{T}$ of $\sigma_{0}$ are connected, as suggested by Figure 2.1.

EXAmPLE 5.15. - The central tile of $\sigma_{3}$ is depicted in Figure 5.6. We use the SRboundary graph (which contains 29 nodes) to build the tile-connectivity graph, it turns out that it is a complete graph, meaning that each subtile intersects all the other subtiles.

The SR-boundary graph is also used to build the tile-refinement-connectivity graph of $\mathcal{J}(2)$. This graph contains three nodes corresponding to the GIFS decomposition of $\mathcal{T}(2)$, namely $\mathbf{h} \mathcal{T}(2), \mathbf{h} \mathcal{J}(3)+\pi(1,0,1), \mathbf{h} \mathcal{T}(3)+\pi(1,1,1)$. We check that $\left[2, \mathbf{h}^{-1} \pi(1,1,1), 3\right]$ is a node of the boundary graph, leading to an edge in the tile-refinement-connectivity graph between $\mathbf{h} \mathcal{T}(2)$ and $\mathbf{h} \mathcal{T}(3)+\pi(1,1,1)$. However, $\left[2, \mathbf{h}^{-1} \pi(1,0,1), 3\right]$ and its symmetric $\left[3,-\mathbf{h}^{-1} \pi(1,0,1), 1\right]$ are not nodes of the SRboundary graph, hence, there is no edge in the tile-refinement-connectivity graph between $\mathbf{h} \mathcal{T}(2)$ and $\mathbf{h} \mathcal{T}(3)+\pi(1,1,1)$. Similarly, neither $\left[3, \mathbf{h}^{-1} \pi(0,1,0), 3\right]$ nor $\left[3,-\mathbf{h}^{-1} \pi(0,1,0), 3\right]$ is a node in the SR-boundary graph, hence, there is no edge between $\mathbf{h} \mathcal{T}(3)+\pi(1,0,1)$ and $\mathbf{h} \mathcal{T}(3)+\pi(1,1,1)$ in the tile-refinement-connectivity graph. We conclude that tile-refinement-connectivity graph of $\mathcal{T}(2)$ contains an isolated node $\mathbf{h} \mathcal{J}(3)+\pi(1,0,1)$. By Theorem 4.9, this proves that $\mathcal{T}(2)$ is not connected, as suggested by the picture in Figure 5.6. The same figure also contains the tile-connectivity graph as well as the tile-refinement-connectivity graph of $\mathcal{T}(2)$.

\subsection{Contact graphs}

In the present section we have to assume that the faces $[\pi(\mathbf{x}), i]$ have a geometric interpretation as polyhedra that induce a tiling of $\mathbb{H}_{c}$. If $\sigma$ is irreducible this is always true in view of Remark 3.3. Indeed, in this case the union of the faces contained in the set $\Gamma_{\text {srs }}$ forms a tiling of the beta-contracting space $\mathbb{H}_{c}$ related to a discrete approximation of this space (see for instance [45]). In the case of reducible substitutions it is not known whether such a geometric interpretation of the faces of $\Gamma_{\text {srs }}$ is always 

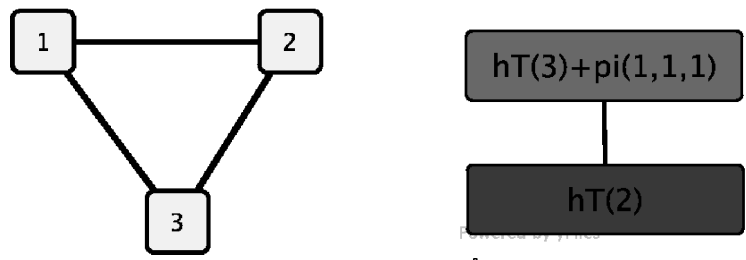

$\mathrm{hT}(3)+\mathrm{pi}(1,0,1)$

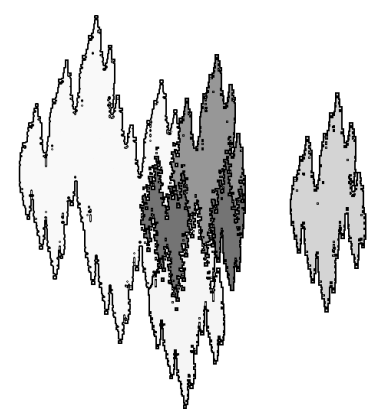

Figure 5.6. The tile-connectivity graph (upper left side) and the tilerefinement-connectivity graph of $\mathcal{T}(2)$ (upper right side) for the substitution $\sigma_{3}(1)=3, \sigma_{3}(2)=23, \sigma_{3}(3)=31223$. The GIFS equation in (2.7) shows that $\mathcal{T}(2)$ can be decomposed into three pieces. The tile-refinementconnectivity graph of $\mathcal{T}(2)$ shows that the piece $\mathbf{h} \mathcal{T}(3)+\pi(1,0,1)$ is disconnected from the other two pieces appearing in the decomposition of $\mathcal{J}(2)$. The patch of tiles in the figure illustrates this situation. The white tiles correspond to $\mathcal{T}(1)$ and $\mathcal{T}(3)$ (these are drawn without subdivision). The subdivision of $\mathcal{T}(2)$ is indicated by the three dark tiles in the figure. The shaded subtile on the right hand side is $\mathbf{h} \mathcal{J}(3)+\pi(1,0,1)$. It has obviously empty intersection with the subtiles $\mathbf{h} \mathcal{T}(3)+\pi(1,1,1)$ and $\mathbf{h} \mathcal{T}(2)$. These tiles are the two darker tiles indicated on the left hand side of the patch (note that $\mathbf{h} \mathcal{T}(2)$, which is the darkest tile, has two components). We deduce that the central tile is not connected.

possible (see [81]). Only for some special instances like for the substitution $\sigma_{1}$ such an interpretation is known (see [80]). In particular, for these examples we can associate a certain polyhedron to each of the faces $[\pi(\mathbf{x}), i]$. However, in the reducible case there is no universal construction known to obtain such polyhedra. They have to be constructed by individual considerations for each of the known instances $(c f .[\mathbf{8 0}, \mathbf{8 1}])$. So in this section we confine ourselves to irreducible unit Pisot substitutions as well as reducible ones admitting a geometric interpretation of their faces.

If the elements of $\Gamma_{\text {srs }}$ can be viewed as polyhedra then images of these faces under the dual substitution $\mathbf{E}_{1}^{*}$ can be regarded as finite unions of such polyhedra which form a subset of $\mathbb{H}_{c}$. Indeed, the dual substitution can be used to define natural approximations of the central tile $\mathcal{T}$ and its subtiles $\mathcal{T}(i)(i \in \mathscr{C})$ in this case. In particular, we set

$$
\mathcal{T}_{m}(i):=\mathbf{h}^{m}\left(\mathbf{E}_{1}^{*}\right)^{m}[\mathbf{0}, i] \quad(i \in \mathscr{Q}) .
$$


Comparing the definition of $\mathbf{E}_{1}^{*}$ with the set equation (2.7) we conclude from the theory of GIFS (see Definition 2.3 and the remarks after it) that

$$
\mathcal{T}(i)=\lim _{m \rightarrow \infty} \mathcal{J}_{m}(i) \quad(i \in \mathscr{C})
$$

holds in Hausdorff metric. For this reason we call $\mathcal{J}_{m}(i)$ the (natural) $m$-th approximation of $\mathcal{T}(i)$.

Contact graphs describe intersections between these approximations of tiles in the self-replicating multiple tiling. Indeed, if the approximations $\mathcal{J}_{m}(i)+\gamma_{1}$ and $\mathcal{J}_{m}(j)+$ $\gamma_{2}$ have nonempty intersection for arbitrarily large $m$ then we say that they have contact.

Possible sets of nodes of contact graphs are finite subsets of the set

$$
\mathscr{G} \times \Gamma_{\mathrm{srs}}=\left\{[i, \gamma, j] ; i \in \mathscr{G},[\gamma, j] \in \Gamma_{\mathrm{srs}}\right\} .
$$

We need the following preliminary definition.

Definition 5.16 (Pre-contact graph). - Let $\delta \subset \mathscr{G} \times \Gamma_{\text {srs }}$ be finite. The pre-contact graph of $\phi$ is denoted by $\tilde{\mathscr{G}}^{(C)}(\phi)$. It is the largest ${ }^{(4)}$ graph such that

1. The nodes of $\tilde{\mathscr{G}}^{(C)}(\phi)$ belong to $\mathfrak{D}$.

2. There is a directed edge from $\left[i_{1}, \gamma_{1}, i_{2}\right]$ to $\left[j_{1}, \gamma_{2}, j_{2}\right]$, labelled by

$$
\eta= \begin{cases}\pi \mathbf{l}\left(p_{1}\right), & \left\langle\mathbf{l}\left(p_{1}\right), \mathbf{v}_{\beta}\right\rangle \leq\left\langle\mathbf{l}\left(p_{2}\right)+\mathbf{x}, \mathbf{v}_{\beta}\right\rangle, \\ \pi \mathbf{l}\left(p_{2}\right)+\gamma, & \text { otherwise. }\end{cases}
$$

where $\mathbf{x} \in \mathbb{Z}^{n}$ is chosen in a way that $\pi(\mathbf{x})=\gamma$ if and only if the relation (5.5) is satisfied.

3. Every node belongs to a walk ending in a node in $\varnothing$.

Proposition 5.17. - For every finite set $\phi \subset \mathscr{G} \times \Gamma_{\mathrm{srs}}$, the pre-contact graph $\tilde{\mathscr{G}}^{(C)}(\phi)$ is finite.

This is proved in [167]. We give a sketch of the proof in Chapter 7.

Now we can state the main properties of pre-contact graphs. The first result is proved in [167]. We recall the proof in Chapter 7.

Proposition 5.18. - Let $\tilde{\mathscr{G}}^{(C)}(\varnothing)$ be a pre-contact graph. The node $[i, \gamma, j]$ of $\tilde{\mathscr{G}}^{(C)}(\phi)$ is the starting point of an infinite walk if and only if the approximations $\mathcal{J}_{m}(i)$ and $\mathcal{J}_{m}(j)+\gamma$ have nonempty intersection for arbitrarily large $m$. As a consequence, $\mathcal{T}(i)$ and $\mathcal{T}(j)+\gamma$ have nonempty intersection in this case, so that $[i, \gamma, j]$ is also a node of the boundary graph $\mathscr{G}^{(B)}(\phi)$.

For a directed graph $G$ denote by $\operatorname{Red}(G)$ the graph emerging from $G$ by successively removing all nodes which are not the starting point of an infinite walk.

(4) The meaning of "largest" is explained in Definition 5.1. 
Definition 5.19 (Contact graph). - Let $\tilde{\mathscr{G}}^{(C)}(\phi)$ be the pre-contact graph of $\varnothing$. Then the contact graph of $\&$ is defined by

$$
\mathscr{G}^{(C)}(\phi):=\operatorname{Red}\left(\tilde{\mathscr{G}}^{(C)}(\phi) \backslash\{[i, \mathbf{0}, i] ; i \in \mathscr{C}\}\right) .
$$

As the nodes $[i, \mathbf{0}, i]$ correspond to the "intersections" $\mathcal{T}(i) \cap \mathcal{T}(i)$ they are not interesting in our context. Indeed, they are governed by the set equation (2.7) of the subtiles of the central tile.

Note that the contact graph $\mathscr{G}^{(C)}(\phi)$ can be algorithmically computed as soon as the set $\varnothing$ is known. Indeed, start with the set of nodes $\phi$. Then recursively increase this set "backards" with nodes $[i, \gamma, j]$ that satisfy conditions (1) and (2) of Definition 5.16. The finiteness of the pre-contact graph stated in Proposition 5.17 ensures that this procedure will eventually stabilize the set of nodes. This yields the pre-contact graph $\tilde{\mathscr{G}}^{(C)}(\varnothing)$. From this final set, the nodes $[i, \mathbf{0}, i]$ and nodes with no outgoing edges have to be removed recursively to obtain $\mathscr{G}^{(C)}(\phi)$. Note that contrary to the boundary graph, the contact graph is constructed by starting from the set $\varnothing$ and going "backwards" in order to find new nodes. This is due to the fact that the contact graph is defined in terms of walks ending in a node in $\phi$.

Proposition 5.18 now implies that when $[i, \gamma, j]$ is a node of the contact graph, not only the tiles $\mathcal{T}(i)$ and $\mathcal{T}(j)+\gamma$ do intersect, but this intersection can already be "seen" in their approximations. Thus $\mathcal{J}(i)$ and $\mathcal{J}(j)+\gamma$ have contact in the selfreplicating tiling. Proposition 5.17 shows that two tiles that have contact are also neighbors. The converse is not true in general.

Let

$$
\oint_{\text {cont }}:=\left\{[i, \gamma, j] \in \mathfrak{D} ; \mathscr{L}_{d-2}([0, i] \cap[\gamma, j])>0\right\}
$$

( $\mathscr{L}_{k}$ denotes the $k$-dimensional Lebesgue measure; the face $[\gamma, j]$ is interpreted as a polygonal face as in Remark 3.3). We define the self-replicating contact graph (SRcontact graph for short) as the contact graph with respect to this set, i.e.,

$$
\mathscr{G}_{\mathrm{srs}}^{(C)}=\mathscr{G}^{(C)}\left(\phi_{\text {cont }}\right) \text {. }
$$

Since $\phi_{\text {cont }}$ is easy to calculate, the SR-contact graph can be constructed as described after Definition 5.19.

THEOREM 5.20. - Let $\sigma$ be an irreducible unit Pisot substitution.

Let $[i, \gamma, j] \in \phi_{\text {cont }}$. Let $C[i, \gamma, j]$ denote the nonempty compact sets that are uniquely defined by the GIFS

$$
C[i, \gamma, j]=\underset{[i, \gamma, j] \stackrel{\eta}{\rightarrow}\left[i_{1}, \gamma_{1}, j_{1}\right] \text { in } \underset{\mathrm{srs}}{g_{\mathrm{sr}}^{(C)}}}{\bigcup} \mathbf{h} C\left[i_{1}, \gamma_{1}, j_{1}\right]+\eta
$$

which is directed by the $S R$-contact graph $\mathscr{G}_{\mathrm{srs}}^{(C)}$. 
If the self-replicating multiple tiling is a tiling, then the boundary of the subsets of the central tile is described by the sets $C[i, \gamma, j]$, i.e.,

$$
\partial \mathcal{T}(i):=\bigcup_{[\gamma, j] \in \Gamma_{\mathrm{srs}}:[i, \gamma, j] \in \mathscr{G}_{\mathrm{srs}}^{(C)}} C[i, \gamma, j]
$$

(here we identify $[i, 0, j]$ with $[j, 0, i]$ in order to capture also the intersections $\mathcal{T}(i) \cap$ $\mathcal{J}(j)$ with $j<i)$.

The proof of this result is given in [167]. The remaining reducible cases mentioned at the beginning of the present section can be treated in the same way. In the following proposition we relate the sets $C[i, \gamma, j]$ defined in Theorem 5.20 to intersections of tiles in the self-replicating tiling.

Proposition 5.21. - Suppose that $\sigma$ is an irreducible primitive unit Pisot substitution that satisfies the tiling property. Let $C[i, \gamma, j]$ be the nonempty compact sets that are defined in Theorem 5.20. Then for every $[i, \gamma, j]$, we have $C[i, \gamma, j] \subset \mathcal{J}(i) \cap$ $(\mathcal{J}(j)+\gamma)$.

Proof. (see [167, Section 4]). - In Proposition 5.18 we saw that the nodes of the contact graph $\mathscr{G}_{\mathrm{srs}}^{(C)}$ form a subgraph of the boundary graph $\mathscr{G}_{\mathrm{srs}}^{(B)}$. Thus

$$
C[i, \gamma, j] \subset B[i, \gamma, j]=\mathcal{T}(i) \cap(\mathcal{T}(j)+\gamma)
$$

and we are done.

By Proposition 5.18, the SR-contact graph is a subgraph of the SR-boundary graph. In $[\mathbf{1 6 7}]$ the SR-contact graphs of the class $(b \geq a \geq 1)$

$$
\begin{aligned}
\sigma(1) & =\underbrace{1 \ldots 1}_{b \text { times }} 2 \\
\sigma(2) & =\underbrace{1 \ldots 1}_{a \text { times }} 3 \\
\sigma(3) & =1
\end{aligned}
$$

of irreducible unit Pisot substitutions have been calculated. The number of their nodes is uniformly bounded by 20 . On the other hand, numerical evidence suggests that the number of nodes of the associated SR-boundary graphs becomes arbitrarily large within this class. This is due to the fact that approximations of central tiles often behave much more nicely than the central tiles themselves. Taking the limit may increase the number of neighbors considerably.

In the following example we deal with the choice $a=b=4$ of the above class. This corresponds to our example $\sigma_{4}$.

EXAMPlE 5.22. - Consider the substitution $\sigma_{4}$. Its SR-contact as well as SRboundary graph is depicted in Figure 5.7. One can see that the SR-contact graph is a proper subgraph of the SR-boundary graph. 


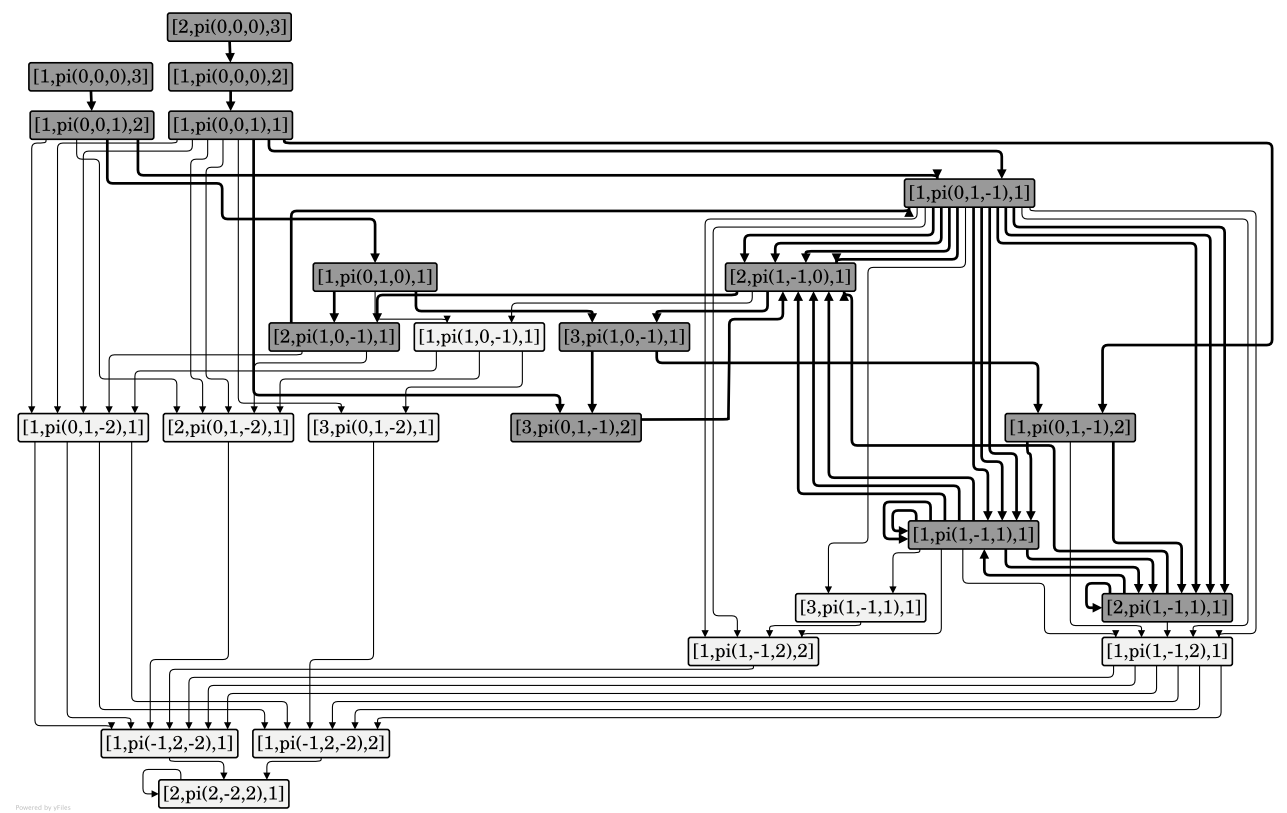

FiguRE 5.7. The dark nodes in the graph depicted in this figure show the SR-contact graph of the substitution $\sigma_{4}$. The whole graph is the SR-boundary graph. In this example the SR-contact graph is a proper subgraph of the SR-boundary graph.

\subsection{Triple points and connectivity of the boundary}

We generalize the definition of boundary graphs in order to describe intersections between three tiles. We will restrict ourselves to the self-replicating multiple tiling, but the definition also holds in the setting of lattice tilings. As we are interested in intersections of three distinct tiles, we set

$\mathfrak{T}=\left\{\left[i, \gamma_{1}, j, \gamma_{2}, k\right] \in \mathscr{G} \times \pi\left(\mathbb{Z}^{n}\right) \times \mathscr{G} \times \pi\left(\mathbb{Z}^{n}\right) \times \mathscr{C} ; \quad[\mathbf{0}, i],\left[\gamma_{1}, i\right],\left[\gamma_{2}, k\right]\right.$ are all distinct $\}$

We first reduce the set of all the possible intersections.

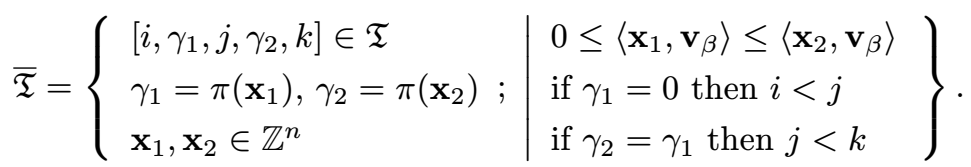

We need to prove that the set $\overline{\mathfrak{T}}$ is enough to describe all the possible intersections of three tiles in a tiling. The following lemma shows that $\overline{\mathfrak{T}}$ contains all possible triple intersections. 
LEMma 5.23. - We define an equivalence relation on $\mathfrak{T}$ by $\left[i, \gamma_{1}, j, \gamma_{2}, k\right] \simeq_{t}$ $\left[i^{\prime}, \gamma_{1}^{\prime}, j^{\prime}, \gamma_{2}^{\prime}, k^{\prime}\right]$ if and only if the sets $\left\{\mathbf{e}_{i}, \mathbf{e}_{j}+\gamma_{1}, \mathbf{e}_{k}+\gamma_{2}\right\}$ and $\left\{\mathbf{e}_{i^{\prime}}, \mathbf{e}_{j^{\prime}}+\gamma_{1}^{\prime}, \mathbf{e}_{k^{\prime}}+\gamma_{2}^{\prime}\right\}$ are equal up to a translation vector. Then the set $\overline{\mathfrak{T}}$ is a quotient set for the equivalence relation $\simeq_{t}$. Indeed, for every $\left[i, \gamma_{1}, j, \gamma_{2}, k\right] \in \mathfrak{T}$, there exists a unique element in $\overline{\mathfrak{T}}$, denoted by $\varphi_{\overline{\mathfrak{T}}}\left(\left[i, \gamma_{1}, j, \gamma_{2}, k\right]\right)$ such that $\left[i, \gamma_{1}, j, \gamma_{2}, k\right] \simeq_{t} \varphi_{\overline{\mathfrak{T}}}\left(\left[i, \gamma_{1}, j, \gamma_{2}, k\right]\right)$.

We denote by $\eta\left(\left[i, \gamma_{1}, j, \gamma_{2}, k\right]\right) \in\left\{\mathbf{0}, \gamma_{1}, \gamma_{2}\right\}$ the translation difference between $\left[i, \gamma_{1}, j, \gamma_{2}, k\right]$ and its representant. If we choose $\mathbf{x} \in \mathbb{Z}^{n}$ in a way that $\eta\left(\left[i, \gamma_{1}, j, \gamma_{2}, k\right]\right)=\pi(\mathbf{x})$ then

$$
\left\langle\mathbf{x}, \mathbf{v}_{\beta}\right\rangle=\min \left\{\mathbf{0},\left\langle\mathbf{x}_{1}, \mathbf{v}_{\beta}\right\rangle,\left\langle\mathbf{x}_{2}, \mathbf{v}_{\beta}\right\rangle\right\} \quad\left(\pi\left(\mathbf{x}_{\ell}\right)=\gamma_{\ell}, \mathbf{x}_{\ell} \in \mathbb{Z}^{n}, \ell=1,2\right) .
$$

The proof of this lemma is a case study, it is done in Chapter 7 .

Coming back to the intersection between three tiles, let $T\left[i, \gamma_{1}, j, \gamma_{2}, k\right]$ denote the intersection

$$
T\left[i, \gamma_{1}, j, \gamma_{2}, k\right]=\mathcal{T}(i) \cap\left(\mathcal{T}(j)+\gamma_{1}\right) \cap\left(\mathcal{T}(k)+\gamma_{2}\right) .
$$

The previous lemma implies that intersections for equivalent triples are equal up to a translation vector, in particular,

$$
T\left[i, \gamma_{1}, j, \gamma_{2}, k\right]=T \varphi_{\overline{\mathfrak{T}}}\left(\left[i, \gamma_{1}, j, \gamma_{2}, k\right]\right)+\eta\left(\left[i, \gamma_{1}, j, \gamma_{2}, k\right]\right) .
$$

Thus we can reduce the study of triple intersections to the set $\overline{\mathfrak{T}}$.

Definition 5.24 (Triple point graph). - The triple point graph of a primitive unit Pisot substitution $\sigma$ is denoted by $\mathscr{G}^{(T)}$. It is the largest ${ }^{(5)}$ graph such that

1. $\left[i, \gamma_{1}, j, \gamma_{2}, k\right] \in \overline{\mathfrak{T}}$ is a node of $\mathscr{G}^{(T)}$, if

$$
\max \left\{\left\|\gamma_{1}\right\|,\left\|\gamma_{2}\right\|\right\} \leq \frac{2 \max \{\|\pi \mathbf{l}(p)\| ;(p, a, s) \in \mathscr{P}\}}{1-\max \left\{\left|\beta^{(\ell)}\right| ; \ell=2, \ldots, d\right\}} .
$$

2. There is a directed edge from $\left[i, \gamma_{1}, j, \gamma_{2}, k\right]$ to $\left[i^{\prime}, \gamma_{1}^{\prime}, j^{\prime}, \gamma_{2}^{\prime}, k^{\prime}\right]$ if and only if there exists $\left[\bar{i}, \overline{\gamma_{1}}, \bar{j}, \overline{\gamma_{2}}, \bar{k}\right] \in \mathfrak{T}$ and $\left(p_{0}, a_{0}, s_{0}\right),\left(p_{1}, a_{1}, s_{1}\right),\left(p_{2}, a_{2}, s_{2}\right) \in \mathscr{P}$ such that

$$
\left\{\begin{array}{l}
{\left[i^{\prime}, \gamma_{1}^{\prime}, j^{\prime}, \gamma_{2}^{\prime}, k^{\prime}\right]=\Phi_{\overline{\mathfrak{T}}}\left[\bar{i}, \overline{\gamma_{1}}, \bar{j}, \overline{\gamma_{2}}, \bar{k}\right]} \\
a_{0}=i \text { and } p_{0} a_{0} s_{0}=\sigma(\bar{i}), \\
a_{1}=j \text { and } p_{1} a_{1} s_{1}=\sigma(\bar{j}), \\
a_{2}=k \text { and } p_{2} a_{2} s_{2}=\sigma(\bar{k}) \\
\mathbf{h} \overline{\gamma_{1}}=\gamma_{1}+\pi \mathbf{l}\left(p_{1}\right)-\pi \mathbf{l}\left(p_{0}\right), \\
\mathbf{h} \overline{\gamma_{2}}=\gamma_{2}+\pi \mathbf{l}\left(p_{2}\right)-\pi \mathbf{l}\left(p_{0}\right) .
\end{array}\right.
$$

This edge is labelled by $\eta \in\left\{\pi \mathbf{l}\left(p_{0}\right), \pi \mathbf{l}\left(p_{1}\right)+\gamma_{1}, \pi \mathbf{l}\left(p_{2}\right)+\gamma_{2}\right\}$ in a way that

$$
\left\langle\mathbf{x}, \mathbf{v}_{\beta}\right\rangle=\min \left\{\left\langle\mathbf{l}\left(p_{0}\right), \mathbf{v}_{\beta}\right\rangle,\left\langle\mathbf{l}\left(p_{1}\right)+\mathbf{x}_{1}, \mathbf{v}_{\beta}\right\rangle,\left\langle\mathbf{l}\left(p_{2}\right)+\mathbf{x}_{2}, \mathbf{v}_{\beta}\right\rangle\right\},
$$

where $\pi(\mathbf{x})=\eta, \pi\left(\mathbf{x}_{1}\right)=\gamma_{1}$, and $\pi\left(\mathbf{x}_{2}\right)=\gamma_{2}$ for some $\mathbf{x}, \mathbf{x}_{1}, \mathbf{x}_{2} \in \mathbb{Z}^{n}$.

3. Every node belongs to an infinite walk starting from a node $\left[i, \gamma_{1}, j, \gamma_{2}, k\right]$ such that $\left[\gamma_{1}, j\right] \in \Gamma_{\text {srs }}$ and $\left[\gamma_{2}, k\right] \in \Gamma_{\text {srs }}$.

(5) The meaning of "largest" is explained in Definition 5.1. 


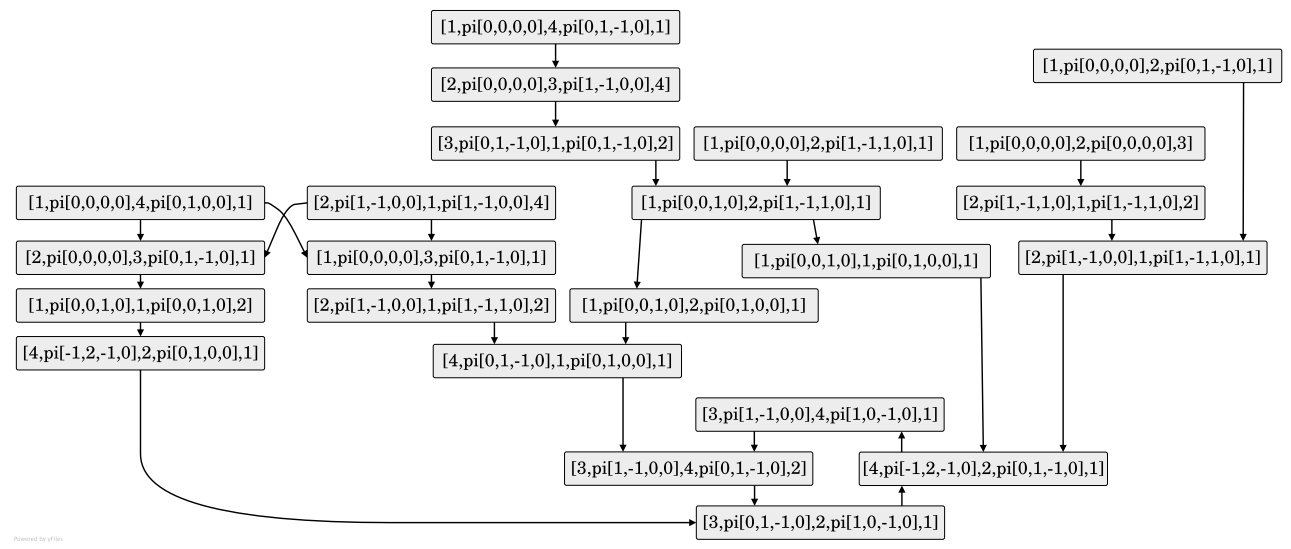

FiguRE 5.8. The triple point graph for the substitution $\sigma_{0}$. There are 23 nodes of the shape $\left[i, \gamma_{1}, j, \gamma_{2}, k\right]$ corresponding to an intersection $\mathcal{T}(i) \cap$ $\mathcal{J}(j)+\gamma_{1} \cap \mathcal{T}(k)+\gamma_{2}$. In this case there are 23 infinite walks in the triple point graph. However, notice that some triple intersections might coincide (see Example 5.27 and Proposition 5.33).

With a treatment similar to the boundary graph, we prove that this graph is finite and characterizes triple points in the self-replicating multiple tiling (see the proof in Chapter 7).

THEOREM 5.25. - Let $\sigma$ be a primitive unit Pisot substitution. Then the following assertions hold.

- The triple point graph is finite.

- Let $\left[i, \gamma_{1}, j, \gamma_{2}, k\right] \in \mathfrak{T}$ be such that $\left[\gamma_{1}, j\right] \in \Gamma_{\mathrm{srs}}$ and $\left[\gamma_{2}, k\right] \in \Gamma_{\mathrm{srs}}$. The intersection $\mathcal{T}(i) \cap\left(\mathcal{T}(j)+\gamma_{1}\right) \cap\left(\mathcal{T}(k)+\gamma_{2}\right)$ is nonempty if and only if $\varphi_{\overline{\mathfrak{T}}}\left(\left[i, \gamma_{1}, j, \gamma_{2}, k\right]\right)$ is a node of the triple point graph.

- A point $\zeta$ belongs to the intersection $\mathcal{T}(i) \cap\left(\mathcal{J}(j)+\gamma_{1}\right) \cap\left(\mathcal{T}(k)+\gamma_{2}\right)$ if and only if there exists an infinite walk starting from $\varphi_{\overline{\mathfrak{T}}}\left(\left[i, \gamma_{1}, j, \gamma_{2}, k\right]\right)$ and labelled by $\left(\eta^{(\ell)}\right)_{\ell \geq 0}$ such that

$$
\zeta=\eta\left(\left[i, \gamma_{1}, j, \gamma_{2}, k\right]\right)+\sum_{\ell \geq 0} \mathbf{h}^{\ell} \eta^{(\ell)} .
$$

The algorithmic construction of $\mathscr{G}^{(T)}$ runs along the same lines as the construction of the SR-boundary graph.

As in the case of boundary graphs (see Definition 5.10) we need to define essentially different walks in a triple point graph.

Definition 5.26. - Two infinite walks in the triple point graph are called essentially different if their labels give rise to h-ary expansions (5.15) of two different points. 
EXAMPLE 5.27. - The triple point graph for the substitution $\sigma_{0}$ is depicted in Figure 5.8. It contains 23 nodes. However, the tiling depicted in Figure 3.3 suggests that there are only 12 triple intersections. The difference between observation and computation comes from the existence of quadruple points: if four pairwise distinct tiles $\mathcal{T}\left(i_{1}\right), \mathcal{T}\left(i_{2}\right)+\gamma_{2}, \mathcal{T}\left(i_{3}\right)+\gamma_{3}, \mathcal{T}\left(i_{4}\right)+\gamma_{4}$ intersect in the same point $\zeta$ then at least three different nodes in the triple point graph correspond to this intersection, namely $\varphi_{\overline{\mathfrak{T}}}\left[i_{1}, \gamma_{2}, i_{2}, \gamma_{3}, i_{3}\right], \varphi_{\overline{\mathfrak{T}}}\left[i_{1}, \gamma_{2}, i_{2}, \gamma_{4}, i_{4}\right]$, and $\varphi_{\overline{\mathfrak{T}}}\left[i_{1}, \gamma_{3}, i_{3}, \gamma_{4}, i_{4}\right]$. Moreover, from each of these nodes there leads away an infinite walk corresponding to $\zeta$ so that there are at least three walks corresponding to $\zeta$.

In the next section, the computation of the quadruple point graph will confirm that this phenomenon explains why there are 23 nodes in the graph but only 12 visible triple points in the tiling.

The triple point graph is useful to detect intersections between the parts of the boundary of a tile $\mathcal{T}(i)$. Indeed, according to Theorem 5.7, $\partial \mathcal{T}(i)$ can be represented as a finite union of pieces of the shape $\mathcal{J}\left(j_{1}\right) \cap\left(\mathcal{J}\left(j_{2}\right)+\gamma\right)$. The boundary-connectivity graph contains information about intersections between these pieces.

Definition 5.28 (Boundary-connectivity graph). - Let $i \in \mathscr{C}$. The boundary-connectivity graph of $\mathcal{T}(i)$ is defined as follows. Its nodes are the sets $\mathcal{T}\left(j_{1}\right) \cap\left(\mathcal{T}\left(j_{2}\right)+\gamma\right)$ with $\left[\gamma, j_{2}\right] \in \Gamma_{\text {srs }}$ that are not reduced to a single point and that appear in the decomposition of the boundary of $\mathcal{T}(i)$ according to Theorem 5.7. Each two of them are connected by an edge if and only if they have nonempty intersection.

Algorithmic construction. According to Theorem 5.7, the nodes of the boundary-connectivity graph of $\mathcal{T}(i)$ are the nodes of the shape $[i, \gamma, j] \in \mathscr{G}_{\text {srs }}^{(B)}$ (again we identify $[i, \mathbf{0}, j]$ and $[j, \mathbf{0}, i])$. We remove from this set the nodes from which there is a unique outgoing infinite walk in the SR-boundary graph. To determine whether there is an edge between $\left[i_{1}, \gamma_{1}, j_{1}\right]$ and $\left[i_{2}, \gamma_{2}, j_{2}\right]$, we notice that among the four pairs $\left[\mathbf{0}, i_{1}\right],\left[\gamma_{1}, j_{1}\right],\left[\mathbf{0}, i_{2}\right],\left[\gamma_{2}, j_{2}\right]$, there are exactly three different pairs, $[\mathbf{0}, i],\left[\gamma_{1}^{\prime}, j_{1}^{\prime}\right]$ and $\left[\gamma_{2}^{\prime}, j_{2}^{\prime}\right]$, say. Then we put an edge between the two nodes if and only if the triple point graph contains the node $\varphi_{\overline{\mathfrak{T}}}\left[i, \gamma_{1}^{\prime}, j_{1}^{\prime}, \gamma_{2}^{\prime}, i_{2}^{\prime}\right]$.

EXAMPLE 5.29. - Let us consider again the substitution $\sigma_{0}$. According to Example 5.11 , the boundary $\partial \mathcal{T}(1)$ is the union of six different pieces that are not reduced to a single point. Their intersections are checked by using the triple point graph, leading to the boundary-connectivity graph of $\mathcal{T}(1)$ depicted in Figure 5.9. The boundaryconnectivity graphs for $\mathcal{T}(2), \mathcal{J}(3)$ and $\mathcal{T}(4)$ are depicted in the same figure.

\subsection{Quadruple points and connectivity of pieces of the boundary}

We build a new graph in order to describe quadruple intersections in the selfreplicating multiple tiling. As mentioned in Example 5.27 this graph is useful in order to decide whether two infinite walks in the triple point graph are essentially different. 

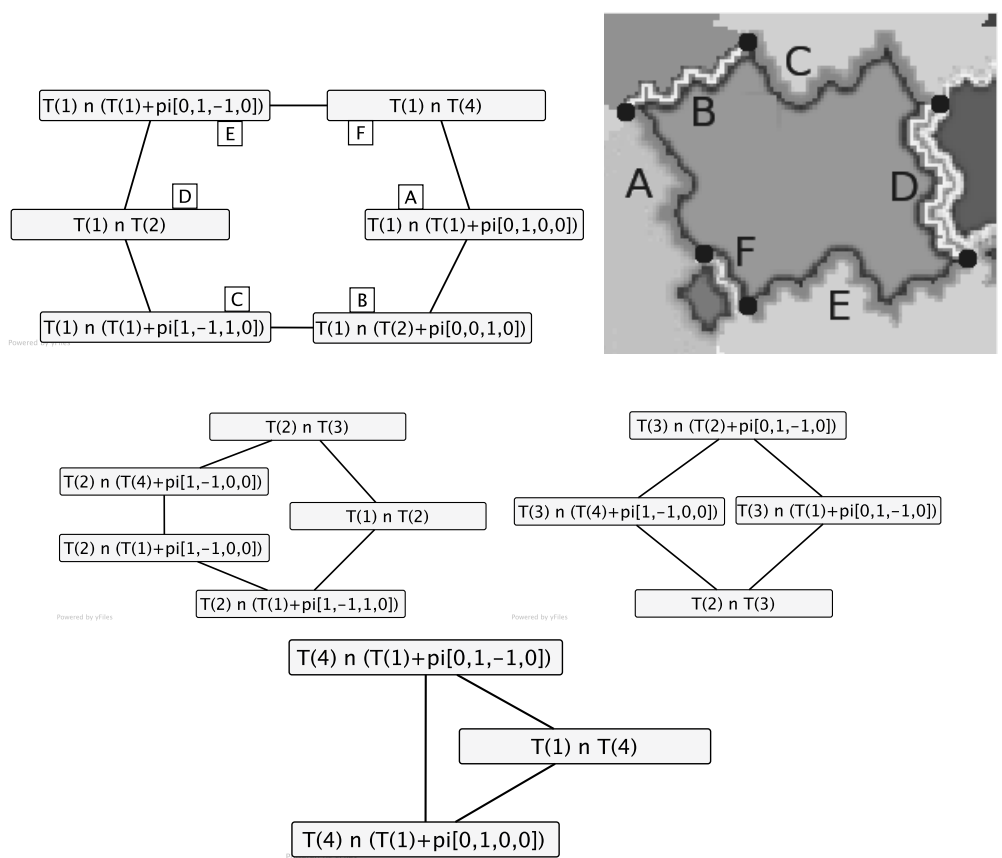

FiguRE 5.9. The boundary connectivity graphs for the subtiles $\mathcal{T}(1)$, $\mathcal{J}(2), \mathcal{T}(3), \mathcal{T}(4)$ associated to the substitution $\sigma_{0}$. Each node corresponds to an intersection $\mathcal{T}\left(j_{1}\right) \cap T\left(j_{2}\right)+\gamma$ that appears in the decomposition of the boundary of $\mathcal{T}(i)$. An edge between two nodes means that the two corresponding pieces in the boundary intersect. Therefore, the first graph confirms that $\mathcal{T}(1)$ can be described with 6 pieces that each have two adjacent pieces, as suggested in the picture of the tile.

Moreover, it will be used for checking the sufficient condition for the disklikeness of subtiles of $\mathcal{T}$ and in the description of their fundamental group. In order to proceed, we need a description of intersections between four tiles in the self-replicating multiple tiling. We set

$$
\begin{array}{r}
\mathfrak{Q}=\left\{\left[i, \gamma_{1}, j, \gamma_{2}, k, \gamma_{3}, l\right] \in \mathscr{G} \times \pi\left(\mathbb{Z}^{n}\right) \times \mathscr{G} \times \pi\left(\mathbb{Z}^{n}\right) \times \mathscr{G} \times \pi\left(\mathbb{Z}^{n}\right) \times \mathscr{G}\right. \\
\left.[\mathbf{0}, i],\left[\gamma_{1}, i\right],\left[\gamma_{2}, k\right]\left[\gamma_{3}, l\right] \text { are all distinct }\right\} .
\end{array}
$$

Similar to the triple point graph, we reduce the set of all possible intersections to a set of unique representants (up to a translation) $\overline{\mathfrak{Q}}$. The mapping from an intersection to its representant is denoted by $\varphi_{\mathfrak{Q}}$. After that we define a quadruple point graph $\mathscr{G}^{(Q)}$ that allows to describe intersections between four distinct tiles in the self-replicating multiple tiling. Precise definitions and proofs are given in Chapter 7. The main result on the quadruple point graph reads as follows. 


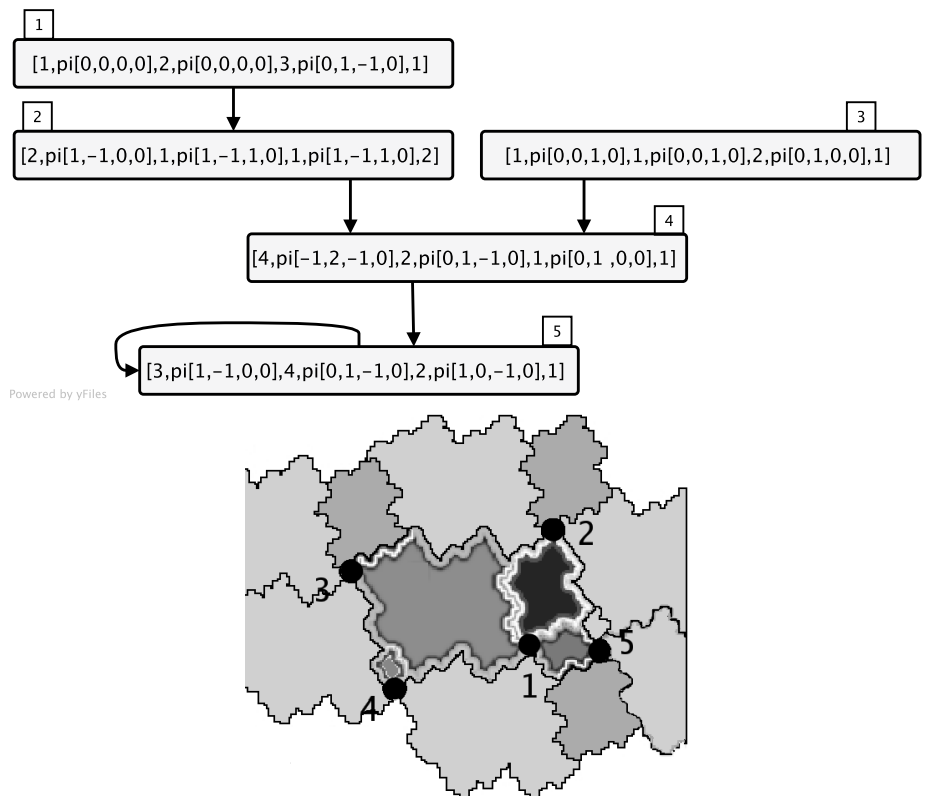

FiguRE 5.10. The quadruple point graph for the substitution $\sigma_{0}$. There are 5 nodes of the shape $\left[i, \gamma_{1}, j, \gamma_{2}, k, \gamma_{3}, l\right]$, corresponding to the intersection $\mathcal{T}(i) \cap \mathcal{T}(j)+\gamma_{1} \cap \mathcal{J}(k)+\gamma_{2} \cap \mathcal{T}(l)+\gamma_{3}$. In this case there are 5 quadruple intersections. Since a unique infinite walk goes out from each node, quadruple intersections are single points. They are all different and marked by black dots on the tiling picture on the left hand side.

TheOREM 5.30. - Let $\sigma$ be a primitive unit Pisot substitution. Then the following assertions hold.

- The quadruple point graph is finite.

- Let $\left[i, \gamma_{1}, j, \gamma_{2}, k, \gamma_{3}, l\right] \in \mathfrak{Q}$. Then $\mathcal{T}(i) \cap\left(\mathcal{T}(j)+\gamma_{1}\right) \cap\left(\mathcal{T}(k)+\gamma_{2}\right) \cap$ $\left(\mathcal{T}(l)+\gamma_{3}\right) \neq \varnothing$ if and only if the quadruple point graph contains the node $\varphi_{\mathfrak{Q}}\left[i, \gamma_{1}, j, \gamma_{2}, k, \gamma_{3}, l\right]$.

- Each point of the intersection $\mathcal{T}(i) \cap\left(\mathcal{T}(j)+\gamma_{1}\right) \cap\left(\mathcal{T}(k)+\gamma_{2}\right) \cap\left(\mathcal{T}(l)+\gamma_{3}\right)$ corresponds to an infinite walk in $\mathscr{G}^{(Q)}$ starting in $\varphi_{\mathfrak{Q}}\left[i, \gamma_{1}, j, \gamma_{2}, k, \gamma_{3}, l\right]$.

EXAMPLE 5.31. - The quadruple point graph for the substitution $\sigma_{0}$ is depicted in Figure 5.10. There are 5 quadruple intersections, as suggested by the illustration of the tiling in this figure. Since a unique infinite walk starts from each node of $\mathscr{G}^{(Q)}$, each node corresponds to an intersection of four tiles which contains one single point. It is easy to see that each infinite walk in the quadruple point graph corresponds to a point that is different from the other. The quadruple points are depicted in Figure 5.10. 
We use the quadruple point graph in order to decompose the pieces of the boundary of each tile. Indeed, by (5.7) the intersections $B[i, \gamma, j]=\mathcal{J}(i) \cap(\mathcal{T}(j)+\gamma)$ are the solution of a GIFS.

DEFINITION 5.32 (Boundary-refinement-connectivity graph). - The nodes of boundary-refinement-connectivity graph of $\mathcal{J}(i) \cap(\mathcal{J}(j)+\gamma)$ are the sets in the union on the right hand side of (5.7) that are not equal to a single point. Each two of them are connected by an edge if and only if they have nonempty intersection.

Algorithmic construction. The nodes of the boundary-refinement-connectivity graph are of the shape $\eta^{(1)}+\mathbf{h}\left(\mathcal{T}\left(i_{1}\right) \cap\left(\mathcal{T}\left(j_{1}\right)+\gamma_{1}\right)\right)$, where $[i, \gamma, j] \stackrel{\eta^{(1)}}{\longrightarrow}\left[i_{1}, \gamma_{1}, j_{1}\right]$ is an edge in the SR-boundary graph. There is an edge between $\eta^{(1)}+\mathbf{h}\left(\mathcal{T}\left(i_{1}\right) \cap\left(\mathcal{T}\left(j_{1}\right)+\gamma_{1}\right)\right)$ and $\eta^{(2)}+\mathbf{h}\left(\mathcal{J}\left(i_{2}\right) \cap\left(\mathcal{J}\left(j_{2}\right)+\gamma_{2}\right)\right)$ in the boundary-refinement-connectivity graph if and only if $\left.\varphi_{\mathfrak{Q}}\left[i_{1}, \gamma_{1}, j_{1}, \mathbf{h}^{-1}\left(\eta^{(2)}-\eta^{(1)}\right), i_{2}, \gamma_{2}+\mathbf{h}^{-1}\left(\eta^{(2)}-\eta^{(1)}\right), j_{2}\right)\right]$ is a node of the quadruple point graph (or of the triple point graph if two of the pairs $\left[\mathbf{0}, i_{1}\right],\left[\gamma_{1}, j_{1}\right]$, $\left[\mathbf{h}^{-1}\left(\eta^{(2)}-\eta^{(1)}\right), i_{2}\right],\left[\gamma^{(2)}+\mathbf{h}^{-1}\left(\eta^{(2)}-\eta^{(1)}, j_{2}\right]\right.$ coincide $)$.

It is often necessary to decide whether a given intersection $B[i, \gamma, j]$ contains at least two points or not. This is equivalent to the existence of two essentially different infinite walks leading away from the node $[i, \gamma, j]$ in the SR-boundary graph. In practice, this can often be decided very easily (see for instance in Example 5.34). However, we want to show that it can always be done algorithmically.

Proposition 5.33. - Consider a node $\mathcal{N}$ in the SR-boundary graph, the triple point graph or the quadruple point graph.

- If an infinite number of infinite walks lead away from the node $\mathcal{N}$, then the intersection of tiles associated with the node $\mathcal{N}$ contains an infinite number of different points.

- If from the node $\mathcal{N}$ there lead away only a finite number of infinite walks, then each of these walks is ultimately periodic. Consequently, the point described by a given walk can be explicitly computed and compared to the others. This allows to decide in a finite time whether the corresponding intersection is a single point or not.

The proof is given in Chapter 7.

\subsection{Application of the graphs to the disklikeness criterion}

Let $\sigma$ be a primitive unit Pisot substitution which satisfies the tiling property. Moreover, assume that the algebraic degree of the dominant eigenvalue $\beta$ of its incidence matrix $\mathbf{M}$ is equal to three. Then, according to Theorem 4.13, checking whether the tile $\mathcal{T}(i)$ is homeomorphic to a disk amounts to checking the following three conditions.

(1) Each boundary-connectivity graph is a simple loop. 
(2) Each boundary-refinement-connectivity graph is either empty or a single node or a line.

(3) Each intersection between three distinct tiles of the self-replicating tiling is either empty or a single point.

EXAMPLE 5.34. - Let us consider again the substitution $\sigma_{0}(1)=112, \sigma_{0}(2)=113$, $\sigma_{0}(3)=4, \sigma_{0}(4)=1$. We want to show for this example that each subtile $\mathcal{T}(i)$ $(1 \leq i \leq 4)$ of the central tile $\mathcal{T}$ of $\sigma_{0}$ is homeomorphic to a closed disk. To this matter we have to check the Items (1), (2) and (3) above.

Checking (1): The boundary-connectivity graphs of $\mathcal{T}(i)(1 \leq i \leq 4)$ are depicted in Figure 5.9. Since they are all simple loops, Item (1) is satisfied.

Checking(2) : Checking the SR-boundary graph depicted in Figure 5.2 we verify the following assertions.

- The SR-boundary graph has 6 nodes having a unique outgoing infinite walk. Each of the corresponding intersections is therefore a single point. Thus the boundary-refinement-connectivity graph of the associated boundary piece is empty.

- The SR-boundary graph has 7 additional nodes having a single outgoing edge leading to several infinite walks some of which are pairwise essentially different. Their boundary-refinement-connectivity graph is thus a single node.

- The SR-boundary graph has 4 nodes having two outgoing edges. Their boundary-refinement-connectivity graph is either a pair of nodes linked by a single edge, or a single node (depending on whether the walks are essentially different or not).

- The SR-boundary graph has 4 remaining nodes that have at least three outgoing edges. These are the nodes $[1, \pi(0,1,-1,0), 1],[1, \pi(1,-1,1,0), 1]$, $[2, \pi(1,-1,0,0), 1]$ and $[1, \pi(0,1,0,0), 1]$. Following $(5.7)$, we obtain the GIFS decomposition of the boundary piece corresponding to the first node. It reads

$$
\begin{aligned}
B[1, \pi(0,1,-1,0), 1] & =\mathbf{h} B[2, \pi(1,-1,0,0), 1] \cup(\mathbf{h} B[2, \pi(1,-1,0,0), 1]+\pi(1,0,0,0)) \\
& \cup \mathbf{h} B[2, \pi(1,-1,0,0), 4] \cup(\mathbf{h} B[1, \pi(0,0,1,0), 2]+\pi(0,1,-1,0)) \\
& \cup(\mathbf{h} B[1, \pi(0,0,1,0), 1]+\pi(0,1,-1,0)) .
\end{aligned}
$$

In this decomposition, the last piece $\mathbf{h} B[1, \pi(0,0,1,0), 1]+\pi(0,1,-1,0)$ is a single point. Therefore, we do not take it into account in the boundaryrefinement-connectivity graph. This latter graph thus has 4 nodes. Intersections between the corresponding pieces of the central tile are checked by using the triple point graph and the quadruple point graph. The result is shown in Figure 5.11. Similarly, $B[1, \pi(1,-1,1,0), 1]$ is made of 5 distinct pieces by the GIFS equation; 2 pieces among these 5 correspond to single points and are not relevant to the boundary-refinement-connectivity graph. Finally $B[1, \pi(0,1,0,0), 1]$ and $B[2, \pi(1,-1,0,0), 1]$ have a GIFS 


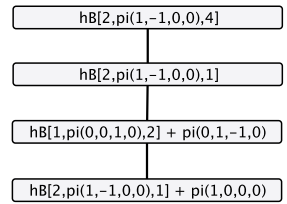

$\mathrm{B}[1, \mathrm{pi}(0,1,-1,0), 1]$

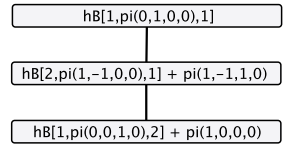

$\mathrm{B}[1, \mathrm{pi}(1,-1,1,0), 1]$

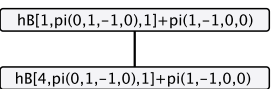

$B[2, p i(1,-1,0,0), 1]$

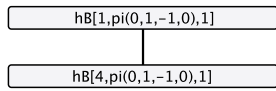

$B[1, p i(0,1,0,0), 1]$

FigURE 5.11. Nontrivial boundary-refinement-connectivity graphs for the substitution $\sigma_{0}$. We draw the boundary-refinement-connectivity graph for all nodes of the SR-boundary graph that have at least three outgoing edges.

decomposition in three pieces each; in each case, one piece of the three pieces is reduced to a single point). Their boundary-refinement graphs are also depicted in Figure 5.11.

Summing up we get that all the boundary-refinement graphs are either empty or single points or lines. Thus Item (2) is satisfied.

Checking (3) : In order to apply Theorem 4.13, we finally have to check that each intersection between three distinct tiles is empty or equal to a single point. Although the triple point graph will be our main tool, we will also use quadruple intersections as an auxiliary tool to show that this item is satisfied by $\sigma_{0}$. Observe that each quadruple intersection is a single point as a unique infinite walk leads away from each node of the quadruple point graph (see Figure 5.10).

We only have to check intersections which correspond to nodes of the triple point graph, as all the other triple intersections are empty. In the triple point graph, all nodes but three have a unique outgoing infinite walk. These exceptional nodes are $[1, \mathbf{0}, 4, \pi(0,1,0,0), 1],[1, \pi(0,0,1,0), 2, \pi(1,-1,1,0), 1]$ and $[2, \pi(1,-1,0,0), 1, \pi(1,-1,0,0), 4]$. As outlined in Proposition 5.33 we show that each of these intersections is a single point by inspecting the quadruple point graph. Indeed, for these three nodes, we write the GIFS decomposition of the corresponding intersection and get the following equations.

$$
\begin{aligned}
& T[1, \mathbf{0}, 4, \pi(0,1,0,0), 1]=\mathbf{h} T[1, \mathbf{0}, 3, \pi(0,1,-1,0), 1] \\
& \cup \mathbf{h} T[2, \mathbf{0}, 3, \pi(0,1,-1,0), 1], \\
& T[2, \pi(1,-1,0,0), 1, \pi(1,-1,0,0), 4]=(\mathbf{h} T[1, \mathbf{0}, 3, \pi(0,1,-1,0), 1]+\pi(0,1,-1,0)) \\
& \cup(\mathbf{h} T[2, \mathbf{0}, 3, \pi(0,1,-1,0), 1]+\pi(0,1,-1,0)), \\
& T[1, \pi(0,0,1,0), 2, \pi(1,-1,1,0), 1]=\mathbf{h} T[1, \pi(0,0,1,0), 2, \pi(0,1,0,0), 1] \\
& \cup \mathbf{h} T[1, \pi(0,0,1,0), 1, \pi(0,1,0,0), 1] .
\end{aligned}
$$

Here we remark that each piece appearing on the right hand side of these equations is equal to a single point, since a unique infinite walk leaves away from the 
corresponding node in the triple point graph. Additionally, we have

$$
T[1, \mathbf{0}, 3, \pi(0,1,-1,0), 1] \cap T[2, \mathbf{0}, 3, \pi(0,1,-1,0), 1]=Q[1, \mathbf{0}, 2, \mathbf{0}, 3, \pi(0,1,-1,0), 1],
$$

and this node appears in the quadruple point graph.

Hence, the intersections $T[1, \mathbf{0}, 3, \pi(0,1,-1,0), 1]$ and $T[2, \mathbf{0}, 3, \pi(0,1,-1,0), 1]$ are equal and correspond to the quadruple intersection $Q[1, \mathbf{0}, 2, \mathbf{0}, 3$, $\pi(0,1,-1,0), 1]$. However, as from each node in the quadruple point graph there leads away only one single infinite walk, $Q[1, \mathbf{0}, 2, \mathbf{0}, 3, \pi(0,1,-1,0), 1]$ is a single point. Thus also the intersection $T[1, \mathbf{0}, 4, \pi(0,1,0,0), 1]$ is a single point. One checks in the same way that the same is true for the two other triple intersections that have more than one outgoing walk in the triple point graph. Thus Item (3) is satisfied.

Summing up we see that the conditions for disklikeness are satisfied. Therefore, each of the subtiles $\mathcal{T}(1), \ldots, \mathcal{T}(4)$ of $\sigma_{0}$ is homeomorphic to a closed disk. It is not hard to check that the same is true for the central tile $\mathcal{J}$.

EXAMPLE 5.35. - For $\sigma_{1}$ a similar reasoning leads to the conclusion that each of the subtiles $\mathcal{T}(1), \ldots, \mathcal{J}(5)$ as well as $\mathcal{T}$ is homeomorphic to a closed disk. From Figure 3.1 we see that in this case there exist even 5 -tuple points. Even if, according to Proposition 5.33, the 5-tuple graph would be needed in order to check that the triple intersections are single points, this can be proved in practice much easier by inspecting the different representations derived from the (finitely many) infinite walks of the triple point graph. 



\section{CHAPTER 6}

\section{EXACT STATEMENTS \\ AND PROOFS OF THE MAIN RESULTS}

In this chapter we give the exact statements of the main results of the present monograph that were already announced in Chapter 4. Moreover, we provide full proofs of all the results.

\subsection{Tiling properties}

In this section we give the complete proofs of Theorem 4.1 and Theorem 4.2.

We need some preparations. Set

$$
\begin{aligned}
R(m, i) & :=\left\{[\gamma, i] \in\left(\mathbf{E}_{1}^{*}\right)^{m}[\mathbf{0}, i] ; \mathbf{h}^{m}(\mathcal{J}(j)+\gamma) \cap \partial \mathcal{J}(i) \neq \varnothing\right\}, \\
S(m, i) & :=\left\{[\gamma, i] \in\left(\mathbf{E}_{1}^{*}\right)^{m}[\mathbf{0}, i]\right\} .
\end{aligned}
$$

Thus, in view of (3.4) the set $S(m, i)$ consists of the addresses of all subtiles contained in the $m$-th subdivision of $\mathcal{T}(i)$. Moreover, $R(m, i)$ contains only those subtiles of the $m$-th subdivision of $\mathcal{T}(i)$ that have nonempty intersection with $\partial \mathcal{T}(i)$. As we will deal with walks in graphs, we will adopt the following notation. If $G$ is a graph and $\nu$ is one of its nodes, we denote by $W_{m}(G, \nu)$ the set of walks in $G$ of length $m$ starting in $\nu$. Furthermore, $W_{m}(G)$ denotes the set of all walks in $G$ of length $m$.

We start with three technical lemmas (recall the definition of the symbol "«" given after equation (3.5)).

Lemma 6.1. - Let $\sigma$ be a primitive unit Pisot substitution that enjoys the tiling property. Then for each $m \in \mathbb{N}$ and each node $\left[i_{1}, \gamma, i_{2}\right]$ of the SR-boundary graph

$$
\left|W_{m}\left(\mathscr{G}_{\mathrm{srs}}^{(B)},\left[i_{1}, \gamma, i_{2}\right]\right)\right| \ll\left|R\left(m, i_{1}\right)\right|
$$

holds uniformly in $m$. 
Proof. - An application of (3.4) yields

$$
\begin{aligned}
& \mathcal{T}\left(i_{1}\right) \cap\left(\mathcal{T}\left(i_{2}\right)+\gamma\right)= \\
& \bigcup_{\left[\delta_{1}, j_{1}\right] \in\left(\mathbf{E}_{1}^{*}\right)^{m}\left[\mathbf{0}, i_{1}\right]} \bigcup_{\left[\delta_{2}, j_{2}\right] \in\left(\mathbf{E}_{1}^{*}\right)^{m}\left[\mathbf{0}, i_{2}\right]}\left(\mathbf{h}^{m}\left(\mathcal{J}\left(j_{1}\right)+\delta_{1}\right) \cap\left(\mathbf{h}^{m}\left(\mathcal{T}\left(j_{2}\right)+\delta_{2}\right)+\gamma\right)\right) .
\end{aligned}
$$

By Theorem 5.7 and the definition of the SR-boundary graph the nonempty intersections in the union on the right hand side of (6.1) are in one-to-one correspondence with the elements of $W_{m}\left(\mathscr{G}_{\mathrm{srs}}^{(B)},\left[i_{1}, \gamma, i_{2}\right]\right)$. Thus it remains to establish an upper bound for the number of these nonempty intersections.

First we claim that $\mathbf{h}^{m}\left(\mathcal{T}\left(j_{1}\right)+\delta_{1}\right) \cap\left(\mathbf{h}^{m}\left(\mathcal{T}\left(j_{2}\right)+\delta_{2}\right)+\gamma\right) \neq \varnothing$ implies that $\left[\delta_{1}, j_{1}\right] \in R(m, i)$. Indeed, the tiling property implies in view of Theorem 5.7 that

$$
\mathbf{h}^{m}\left(\mathcal{T}\left(j_{1}\right)+\delta_{1}\right) \cap\left(\mathbf{h}^{m}\left(\mathcal{T}\left(j_{2}\right)+\delta_{2}\right)+\gamma\right) \subset \mathcal{J}\left(i_{1}\right) \cap\left(\mathcal{T}\left(i_{2}\right)+\gamma\right) \subset \partial \mathcal{T}\left(i_{1}\right) .
$$

Thus $\mathbf{h}^{m}\left(\mathcal{J}\left(j_{1}\right)+\delta_{1}\right) \cap \partial \mathcal{T}\left(i_{1}\right) \neq \varnothing$ and the claim is proved. Thus for the first part of the intersections in (6.1) there are $\left|R\left(m, i_{1}\right)\right|$ choices.

Moreover, by the local finiteness of the self-replicating multiple tiling there is a constant $c_{1}$ with the following property. For each fixed set $\mathbf{h}^{m}\left(\mathcal{J}\left(j_{1}\right)+\delta_{1}\right)$ there are at most $c_{1}$ different sets of the shape $\mathbf{h}^{m}\left(\mathcal{T}\left(j_{2}\right)+\delta_{2}\right)+\gamma$ such that $\mathbf{h}^{m}\left(\mathcal{T}\left(j_{1}\right)+\delta_{1}\right) \cap$ $\left(\mathbf{h}^{m}\left(\mathcal{T}\left(j_{2}\right)+\delta_{2}\right)+\gamma\right) \neq \varnothing$. Summing up this yields the desired bound.

Lemma 6.2. - Let $\sigma$ be a primitive unit Pisot substitution that enjoys the tiling property. Then there exists $m_{0} \in \mathbb{N}$ such that

$$
\left|R\left(m_{0}, i\right)\right|<\left|S\left(m_{0}, i\right)\right|
$$

holds for each $i \in \mathscr{C}$.

Proof. - Note that $\mathbf{h}$ is a uniform contraction. Thus for each $j \in \mathscr{G}$ we have $\operatorname{diam}\left(\mathbf{h}^{m} \mathcal{J}(j)\right) \rightarrow 0$ for $m \rightarrow \infty$. As $\operatorname{int}(\mathcal{J}(i)) \neq \varnothing$ there is some $m_{0}(i)$ such that at least one tile in the $m_{0}(i)$-th subdivision of $\mathcal{T}(i)$ has empty intersection with $\partial \mathcal{T}(i)$. Taking $m_{0}:=\max _{i \in \mathscr{C}} m_{0}(i)$ proves the result.

Let $m_{0}$ be given as in Lemma 6.2. Setting $c_{2}:=\max _{i \in \mathscr{Q}}\left|S\left(m_{0}, i\right)\right|$ this lemma yields

$$
\frac{\left|R\left(m_{0}, i\right)\right|}{\left|S\left(m_{0}, i\right)\right|} \leq \frac{c_{2}-1}{c_{2}} .
$$

We can use this to obtain the following result by induction.

Lemma 6.3. - Let $\sigma$ be a primitive unit Pisot substitution that enjoys the tiling property and choose $m_{0}$ as in Lemma 6.2. Then

$$
\frac{\left|R\left(k m_{0}, i\right)\right|}{\left|S\left(k m_{0}, i\right)\right|} \leq\left(\frac{c_{2}-1}{c_{2}}\right)^{k}
$$

holds for each $i \in \mathscr{C}$ and each $k \in \mathbb{N}$. 
Proof. - We use induction on $k$. For $k=1$ the result follows from (6.2). Assume that (6.3) is true for a given $k$. Let $T$ be a subtile of $\mathcal{J}(i)$ associated to an element of $S\left(k m_{0}, i\right)$. If $T \notin R\left(k m_{0}, i\right)$ then none of the subtiles forming the $m_{0}$-th subdivision of $T$ intersects $\partial \mathcal{T}(i)$. Thus none of the corresponding elements of $S\left((k+1) m_{0}, i\right)$ is contained in $R\left((k+1) m_{0}, i\right)$. If, on the other hand, $T \in R\left(k m_{0}, i\right)$ then let $N$ be the number of subtiles of the $m_{0}$-th subdivision of $T$. By $(6.2)$ at most $\left(c_{2}-1\right) N / c_{2}$ of them are contained in $R\left((k+1) m_{0}, i\right)$. Summing up we get the result.

The last three lemmas imply the following result which will be the key to prove one direction of the equivalences in Theorem 4.1.

Corollary 6.4. - Let $\sigma$ be a primitive unit Pisot substitution that enjoys the tiling property. Then there is a constant $c_{3} \in(0,1)$ such that

$$
\left|W_{m}\left(\mathscr{G}_{\mathrm{srs}}^{(B)}\right)\right| \ll\left(c_{3} \beta\right)^{m}
$$

holds uniformly for each $m \in \mathbb{N}$.

Proof. - By (3.5) we have $|S(m, i)| \ll \beta^{m}$. Thus, setting $c_{3}=\left(\frac{c_{2}-1}{c_{2}}\right)^{\left(1 / m_{0}\right)}$, this implies together with Lemmas 6.1 and 6.3 that

$$
\left|W_{n}\left(\mathscr{G}_{\mathrm{srs}}^{(B)}\right)\right| \ll \max _{i \in \mathscr{C}}|R(m, i)| \ll c_{3}^{m} \max _{i \in \mathscr{G}}|S(m, i)| \ll\left(c_{3} \beta\right)^{m} .
$$

Proof of Theorem 4.1. - We start with the proof of equivalence (1) in Theorem 4.1. Assume that $\mu<\beta$. By Theorem 5.7 the intersections $B[i, \gamma, j]=\mathcal{J}(i) \cap(\mathcal{J}(j)+\gamma)$ are given by the GIFS in (5.7) which is directed by the SR-boundary graph.

As $\mu$ is the modulus of the largest eigenvalue of the incidence matrix of $\mathscr{G}_{\mathrm{srs}}^{(B)}$, there are $\ll \mu^{m}$ walks of length $m$ starting at $[i, \gamma, j]$ in $\mathscr{G}_{\mathrm{srs}}^{(B)}$. Thus, iterating (5.7) for $m$ times and taking the Lebesgue measure we get $(d$ is the algebraic degree of $\beta$ )

$$
\begin{aligned}
\mathscr{L}_{d-1}(\mathcal{T}(i) \cap(\mathcal{T}(j)+\gamma)) & \ll \mu^{m} \mathcal{L}_{d-1}\left(\mathbf{h}^{m}\left(\mathcal{T}\left(i^{\prime}\right) \cap\left(\mathcal{T}\left(j^{\prime}\right)+\gamma^{\prime}\right)\right)\right) \\
& \ll\left(\frac{\mu}{\beta}\right)^{m} \mathscr{L}_{d-1}\left(\mathcal{J}\left(i^{\prime}\right) \cap\left(\mathcal{J}\left(j^{\prime}\right)+\gamma^{\prime}\right)\right),
\end{aligned}
$$

where $\mathcal{T}\left(i^{\prime}\right) \cap\left(\mathcal{J}\left(j^{\prime}\right)+\gamma\right)$ is one of the intersections in the self-replicating multiple tiling having largest Lebesgue measure. Since $\mu<\beta$ and $m$ can be arbitrarily large this yields $\mathscr{L}_{d-1}(\mathcal{T}(i) \cap(\mathcal{T}(j)+\gamma))=0$. Thus as $\mathscr{L}_{d-1}(\mathcal{T}(i))>0$ there is a subset of $\mathcal{T}(i)$ of positive measure that is covered only once by the elements of the collection $\left\{\mathcal{J}(j)+\gamma ;[\gamma, j] \in \Gamma_{\text {srs }}\right\}$. The self-replicating multiple tiling is therefore a tiling.

Assume now that the self-replicating multiple tiling is a tiling. Let $\mathbf{B}^{m}=\left(b_{r s}^{(m)}\right)$ be the $m$-th power of the incidence matrix of the SR-boundary graph. Then Corollary 6.4 implies that $b_{r s}^{(m)} \ll\left(c_{3} \beta\right)^{m}$. Thus the Gershgorin Circle Theorem yields that the modulus of each eigenvalue of $\mathbf{B}^{m}$ is $\ll\left(c_{3} \beta\right)^{m}$ where the implied constant is uniform in $m$. Since eigenvalues are multiplicative, the modulus of each eigenvalue of $\mathbf{B}$ is bounded by $c_{3} \beta$ which is strictly less than $\beta$ as $c_{3}<1$ and we are done. 
To prove equivalence (2) we note that for irreducible unit Pisot substitutions it is shown in [101] that the lattice multiple tiling is a tiling if and only if the selfreplicating multiple tiling is a tiling.

Finally, the proof of (3) runs exactly along the same lines as the proof of (1). We just have to replace the SR-boundary graph by the lattice boundary graph.

The proof of Theorem 4.2 now follows immediately.

Proof of Theorem 4.2. - We first prove (1). Assume that $\mu^{\prime}<\beta$. We can show that this implies that the self-replicating multiple tiling is a tiling exactly in the same way as in the proof of Theorem 4.1. Just use Theorem 5.20 here whenever Theorem 5.7 was used in the SR-boundary graph setting.

To prove the converse recall that Proposition 5.18 shows that the SR-contact graph is a subgraph of the SR boundary graph. Thus this follows from Theorem 4.1.

Equivalence (2) is proved by the same reasoning as equivalence (2) of Theorem 4.1.

REMARK 6.5. - It is not hard to show that $\mu=\mu^{\prime}$, i.e., the modulus of the dominant eigenvalue is the same for the incidence matrices of both, the SR-boundary graph and the SR-contact graph.

\subsection{Dimension of the boundary of the subtiles $\mathcal{T}(i)(i \in \mathscr{C})$}

The main reference for the proof of Theorem 4.4 is [167, Section 5]. In this paper the GIFS description of the boundaries $\partial \mathcal{T}(i)(i \in \mathscr{Q})$ in terms of the SR-contact graph contained in Theorem 5.20 is used in order to compute the box-counting dimensions $\operatorname{dim}_{B}(\mathcal{J}(i))$ and $\operatorname{dim}_{B}(\mathcal{T})$ for irreducible unit Pisot substitutions. This leads to formulas for the box-counting dimension containing eigenvalues of the SR-contact graph. By using Theorem 5.7 instead of Theorem 5.20 in the same proof we obtain Theorem 4.4 for irreducible unit Pisot substitutions. The reducible case of Theorem 4.4 is shown in exactly the same way as the irreducible case. Indeed, everything we need in the proof is a GIFS decomposition of the boundary. This is given by the SR-boundary graph in the irreducible as well as in the reducible case. The formula of the box-counting dimension including the contact graph works in the reducible cases only if the contact graph is defined (see Section 5.4 for details).

Summing up, all details for the proof of Theorem 4.4 are contained in [167, Section 5]. We thus refrain from proving this theorem here. 


\subsection{Inner points of $\mathcal{T}$ and the geometric property $(\mathrm{F})$}

In Section 3.1.2 we have introduced the so-called geometric property $(F)$. Now we want to dwell upon the relation of this property to the locus of $\mathbf{0}$ in the central tile. In particular, we shall give the proofs of Theorems 4.6 and 4.7.

Let int* $(\mathcal{J})$ be the set of exclusive inner points of the patch $\mathcal{T}=\mathcal{J}(1) \cup \cdots \cup \mathcal{J}(n)$ in the self-replicating tiling (for a definition of exclusive point see Section 4.4).

Proof of Theorem 4.6. - Assume first that the geometric property (F) holds. Then (3.8) implies that for all $[\gamma, i] \in \Gamma_{\text {srs }}, \gamma$ has a finite $\mathbf{h}$-ary representation $\gamma=\mathbf{h}^{-m} \pi \mathbf{l}\left(p_{-m}\right)+\cdots+\mathbf{h}^{-1} \pi \mathbf{l}\left(p_{-1}\right)$, where $\left(p_{k}, i_{k}, s_{k}\right)_{-m \leq k<0}$ is a finite walk in the prefix-suffix graph that ends at $i=i_{0}$.

Let $\alpha \in \mathcal{J}(i)+\gamma$ with $[\gamma, i] \in \Gamma_{\text {srs }}$. Then, by considering the $\mathbf{h}$-ary representation of $\alpha-\gamma$ given in (2.8), we obtain

$$
\alpha \in \mathcal{T}(i)+\gamma \quad \alpha=\sum_{k=-m}^{\infty} \mathbf{h}^{k} \pi \mathbf{l}\left(p_{k}\right)
$$

where $\left(p_{k}, i_{k}, s_{k}\right)_{k \geq-m}$ is the labelling of a walk in the prefix-suffix graph satisfying $i_{0}=i$.

Suppose that $\mathbf{0} \notin \operatorname{int}^{*}(\mathcal{T})$. Then $\mathbf{0}$ is not an exclusive point of the patch $\mathcal{T}=$ $\mathcal{T}(1) \cup \cdots \cup \mathcal{T}(n)$. But since $\mathcal{T}$ is a patch of the self-replicating multiple tiling, there exists $[\gamma, i] \in \Gamma_{\text {srs }}$ with $\gamma \neq \mathbf{0}$ such that $\mathbf{0} \in \mathcal{J}(i)+\gamma$.

In view of (6.4) the existence of $[\gamma, i] \in \Gamma_{\text {srs }}$ with $\gamma \neq \mathbf{0}$ and $\mathbf{0} \in \mathcal{J}(i)+\gamma$ is equivalent to the existence of a representation of the shape

$$
\mathbf{0}=\sum_{j=-m}^{\infty} \mathbf{h}^{j} \pi \mathbf{l}\left(p_{j}\right) \text {. }
$$

Multiplying this equation by $\mathbf{h}^{-k}$ yields $\mathbf{0}=\sum_{j=-m}^{\infty} \mathbf{h}^{j-k} \pi \mathbf{l}\left(p_{j}\right)$. Applying (6.4) again this implies that $\mathbf{0} \in \mathcal{T}\left(i_{k}\right)+\sum_{\ell=1}^{m+k} \mathbf{h}^{-\ell} \pi \mathbf{l}\left(p_{k-\ell}\right)$ holds for each $k \in \mathbb{N}$, a contradiction to the local finiteness of self-replicating multiple-tiling $\Gamma_{\text {srs }}$ (note that the representations in (3.8) are unique; thus for each $k$ the sum $\sum_{\ell=1}^{m+k} \mathbf{h}^{-\ell} \pi \mathbf{l}\left(p_{k-\ell}\right)$ represents a different element). Thus $\mathbf{0}$ is an exclusive inner point of $\mathcal{J}$. However, since the strong coincidence condition is satisfied, this implies together with Theorem 2.10, that there is a set of positive measure around $\mathbf{0}$, which is covered only once by the self-replicating multiple tiling. Hence, the self-replicating multiple tiling is a tiling.

Now assume on the contrary that the geometric property $(\mathrm{F})$ does not hold. This implies that there exists

$$
\left[\gamma_{0}, i_{0}\right] \in \Gamma_{\text {srs }} \backslash \bigcup_{m \geq 0}\left(\mathbf{E}_{1}^{*}\right)^{m}(\mathcal{U}) .
$$

Note that this assures that $\gamma_{0} \neq \mathbf{0}$. Since Proposition 3.5 implies that $\mathbf{E}_{1}^{*}\left(\Gamma_{\mathrm{srs}}\right)=\Gamma_{\mathrm{srs}}$ we can define a sequence $\left(\left[\gamma_{k}, i_{k}\right]\right)_{k \geq 1}$ of elements of $\Gamma_{\text {srs }}$ with

$$
\left[\gamma_{k}, i_{k}\right] \in \mathbf{E}_{1}^{*}\left(\left[\gamma_{k+1}, i_{k+1}\right]\right) \quad(k \geq 0) .
$$


By the definition of $\mathbf{E}_{1}^{*}$ (recall in particular the contraction ratio of the mapping $\mathbf{h}$ given in (2.2)) this yields

$$
\left\|\gamma_{k+1}\right\| \leq \max \left\{\left|\beta^{(j)}\right| ; j=2, \ldots, d\right\}|| \gamma_{k} \|+\max \{\pi \mathbf{l}(p) ;(p, a, s) \in \mathscr{P}\} .
$$

Since $\Gamma_{\text {srs }}$ is a Delaunay set by Proposition 3.7 there exists $K \in \mathbb{N}$ such that

$$
\left\|\gamma_{k}\right\| \leq \frac{\max \{\pi \mathbf{l}(p) ;(p, a, s) \in \mathscr{P}\}}{1-\max \left\{\left|\beta^{(\ell)}\right| ; \ell=2, \ldots, d\right\}} \quad \text { for } k \geq K
$$

(note that without the Delaunay property we would get a slightly weaker bound). Again because $\Gamma_{\text {srs }}$ is a Delaunay set there exist only finitely many $[\gamma, i] \in \Gamma_{\text {srs }}$ satisfying the inequality in (6.5). For the sequence $\left(\left[\gamma_{k}, i_{k}\right]\right)_{k \geq 0}$ this means that there is a $K^{\prime}>K$ and a $p>0$ such that

$$
\left[\gamma_{K^{\prime}}, i_{K^{\prime}}\right]=\left[\gamma_{K^{\prime}+p}, i_{K^{\prime}+p}\right]
$$

Now observe the following facts. Firstly, $\gamma_{K^{\prime}} \neq 0$ because otherwise $\gamma_{0} \in$ $\bigcup_{m>0} \mathbf{E}_{1}^{* m}(\mathcal{U})$ would hold contrary to our assumption. Secondly, by (6.6) and by the definition of $\mathbf{E}_{1}^{*}$ the (nonzero) element $\gamma_{K^{\prime}}$ admits the representation

$$
\gamma_{K^{\prime}}=\mathbf{h}^{-p} \gamma_{K^{\prime}}+\mathbf{h}^{-p} p_{K^{\prime}}+\cdots+\mathbf{h}^{-1} p_{K^{\prime}+p-1} .
$$

However, by the definition of the zero-expansion graph $\mathscr{G}^{(\mathbf{0})}$ this is equivalent to the existence of a loop

$$
\gamma_{K^{\prime}} \rightarrow \gamma_{K^{\prime}+p-1} \rightarrow \cdots \rightarrow \gamma_{K^{\prime}+1} \rightarrow \gamma_{K^{\prime}}
$$

in $\mathscr{G}^{(\mathbf{0})}$. In view of Proposition 5.2 this implies that $\mathbf{0} \in \mathcal{J}\left(i_{K^{\prime}}\right)+\gamma_{K^{\prime}}$. Since $\gamma_{K^{\prime}} \neq \mathbf{0}$ this yields $\mathbf{0} \notin \operatorname{int}^{*}(\mathcal{T})$.

Proof of Theorem 4.7. - The fact that the zero-expansion graph only contains nodes of the shape $[\mathbf{0}, i](i \in \mathscr{G})$ is equivalent to $\mathbf{0} \in \operatorname{int}^{*}(\mathcal{J})$ by Proposition 5.2. But by Theorem 4.6, $\mathrm{O} \in \operatorname{int}^{*}(\mathcal{J})$ is equivalent to the geometric property $(\mathrm{F})$ and we are done.

\subsection{Connectivity}

We need the following definitions.

Definition 6.6. - Let $\left\{Q_{1}, \ldots, Q_{\nu}\right\}$ be a finite collection of sets.

- We say that $\left\{Q_{1}, \ldots, Q_{\nu}\right\}(\nu \geq 1)$ forms a finite chain joining $Q_{1}$ and $Q_{\nu}$ if $Q_{i} \cap Q_{i+1} \neq \varnothing$ for each $i \in\{1, \ldots, \nu-1\}$.

- We say that $\left\{Q_{1}, \ldots, Q_{\nu}\right\}(\nu \geq 1)$ forms a regular chain, if $\left|Q_{i} \cap Q_{i+1}\right|=1$ for each $i \in\{1, \ldots, \nu-1\}$ and $Q_{i} \cap Q_{j}=\varnothing$ if $|i-j| \geq 2$.

- We say that $\left\{Q_{1}, \ldots, Q_{\nu}\right\}(\nu \geq 2)$ forms a circular chain, if $\left|Q_{i} \cap Q_{i+1}\right|=1$ for each $i \in\{1, \ldots, \nu-1\},\left|Q_{\nu} \cap Q_{1}\right|=1$ and $Q_{i} \cap Q_{j}=\varnothing$ if $2 \leq|i-j| \leq \nu-2$.

In $[\mathbf{1 2 1}]$ the following result on the connectivity of GIFS was shown. 
Lemma 6.7 ([121, Theorem 4.1]). - Let $S_{1}, \ldots, S_{q}$ be the solution of a GIFS with graph $G(V, E)$ and contractions $F_{e}(e \in E)$ and set

$$
\mathcal{E}_{i}:=\left\{F_{e}\left(S_{j}\right) ; \text { e is an edge from } i \text { to } j \text { in } G(V, E)\right\} .
$$

Then $S_{j}$ is a locally connected continuum (or a single point) for each $j \in\{1, \ldots, q\}$ if and only if for each $i \in\{1, \ldots, q\}$ and each pair $X_{1}, X_{2} \in \mathcal{E}_{i}$ there exists a finite chain $\mathscr{C}$ in $\mathcal{E}_{i}$ joining $X_{1}$ and $X_{2}$.

We can use Lemma 6.7 in order to derive the criterion given in Chapter 4 for the connectivity of $\mathcal{T}(i)$.

Proof of Theorem 4.9. - Let $i \in \mathscr{G}$ and let $\mathcal{E}_{i}$ be the set of all tiles whose union is equal to $\mathcal{T}(i)$, i.e.,

$$
\mathcal{E}_{i}:=\{\mathbf{h} \mathcal{T}(j)+\gamma ; \exists(p, i, s), \sigma(j)=p i s \text { and } \gamma=\pi(\mathbf{l}(p))\} .
$$

By definition, a pair of tiles $\mathbf{h} \mathcal{J}\left(j_{1}\right)+\gamma_{1}, \mathbf{h} \mathcal{T}\left(j_{2}\right)+\gamma_{2} \in \mathcal{E}_{i}$ intersects if and only if the tile-refinement-connectivity graph of $\mathcal{T}(i)$ contains an edge between $\mathbf{h} \mathcal{T}\left(j_{1}\right)+\gamma_{1}$ and $\mathbf{h} \mathcal{J}\left(j_{2}\right)+\gamma_{2}$.

Hence, for each pair ef elements $\mathbf{h} \mathcal{J}\left(j_{1}\right)+\gamma_{1}, \mathbf{h} \mathcal{J}\left(j_{2}\right)+\gamma_{2} \in \mathcal{E}_{i}$ there exists a finite chain of elements of $\mathcal{E}_{i}$ joining $\mathbf{h} \mathcal{T}\left(j_{1}\right)+\gamma_{1}$ and $\mathbf{h} \mathcal{T}\left(j_{2}\right)+\gamma_{2} \in \mathcal{E}_{i}$ if and only if the tile-refinement-connectivity graph of $\mathcal{J}(i)$ is connected. As $\mathcal{T}(i)(i \in \mathscr{G})$ are the solution of the GIFS (2.7), the assertion in Theorem 4.9 concerning the sets $\mathcal{T}(i)$ now follows from Lemma 6.7 (note that the case that $\mathcal{T}(i)$ is a single point is excluded, since $\mathcal{T}(i)$ is the closure of its interior).

The assertion in Theorem 4.9 concerning $\mathcal{T}$ is now easy to establish. What we proved until now ensures that the $\mathcal{J}(i)$ are connected. The connectivity of the tileconnectivity graph ensures that the connected sets $\mathcal{T}(i)(i \in \mathscr{C})$ form a finite chain. Thus their union is connected by a standard result from plane topology.

\subsection{Homeomorphy to a closed disk}

6.5.1. A necessary condition coming from the lattice tiling property. - We start with a very simple criterion that allows to conclude that a certain central tile $\mathcal{J}$ is not homeomorphic to a closed disk. We need the following lemma, which is proved in Bandt and Gelbrich [32, Section 5] in a more general context.

Lemma 6.8 (see [32, Lemma 5.1]). - Let $\sigma$ be a primitive unit Pisot substitution whose dominant eigenvalue has degree 3. Suppose that the central tile $\mathcal{T}$ admits a lattice tiling of $\mathbb{H}_{c} \cong \mathbb{R}^{2}$ with respect to the lattice $\Gamma_{\text {lat. }}$. Suppose further that $\mathcal{T}$ is homeomorphic to a closed disk. Then the central tile $\mathcal{T}$ has at most eight neighbors in the lattice tiling. Moreover, it has at most six neighbors $\gamma$ with the property

$$
|\mathcal{J} \cap(\mathcal{J}+\gamma)|>1 \text {. }
$$

Together with Proposition 5.12 this lemma immediately implies the following simple criterion for a central tile to be not homeomorphic to a closed disk 
THEOREM 6.9. - Let $\sigma$ be a primitive unit Pisot substitution whose dominant eigenvalue has degree 3. Suppose that the central tile $\mathcal{T}$ admits a lattice tiling with respect to the lattice $\Gamma_{\text {lat }}$.

The central tile $\mathcal{T}$ is not homeomorphic to a closed disk if at least one of the following assertions is true.

- There exist elements $\gamma_{1}, \ldots, \gamma_{5} \in \mathbb{H}_{c}$ with $\gamma_{\ell} \neq \pm \gamma_{m}$ for $\ell \neq m$ such that the lattice boundary graph contains a state of the shape $\left[i_{k}, \gamma_{k}, j_{k}\right]$ with $\left[\gamma_{k}, j_{k}\right] \in \Gamma_{\text {lat }}$ for each $k \in\{1, \ldots, 5\}$.

- There exist elements $\gamma_{1}, \ldots, \gamma_{4} \in \mathbb{H}_{c}$ with $\gamma_{\ell} \neq \pm \gamma_{m}$ for $\ell \neq m$ such that the following property holds. For each $k \in\{1, \ldots, 4\}$ there exist (at least) two essentially different walks in the lattice boundary graph that start in nodes of the shape $\left[i_{k}, \gamma_{k}, j_{k}\right],\left[i_{k}^{\prime}, \gamma_{k}, j_{k}^{\prime}\right]$ with $\left[\gamma_{k}, j_{k}\right],\left[\gamma_{k}, j_{k}^{\prime}\right] \in \Gamma_{\text {lat }}$ (here $i_{k}=i_{k}^{\prime}$ as well as $j_{k}=j_{k}^{\prime}$ is possible; thus the two walks may also start in the same node).

REMARK 6.10. - Note that $[i, \gamma, j] \in \mathscr{G}_{\text {lat }}^{(B)}$ implies that $\mathcal{T} \cap \mathcal{T}+\gamma \neq \varnothing$ and $\mathcal{T} \cap \mathcal{T}-\gamma \neq$ $\varnothing$. That is why each $\gamma_{\ell}$ in the statement of the theorem is related to two different neighbors of $\mathcal{T}$.

The conditions of Theorem 6.9 can obviously be checked algorithmically. Thus Theorem 4.11 follows. Examples for the application of this result are given in Example 5.13. Note that in general Proposition 5.33 is needed in order to decide whether two walks are essentially different.

6.5.2. Preliminary results on GIFS. - In a next step we wish to give a criterion for a GIFS to be homeomorphic to a closed disk. To this matter we need some preparations.

Let $S_{1}, \ldots, S_{q}$ be the solution of a GIFS directed by a finite directed graph $G=$ $G(V, E)$ with $V:=\{1, \ldots, q\}$ where to each edge $e \in E$ there is associated a contraction $F_{e}$. As before, we denote the set of walks in $G$ starting at $i \in V$ and having length $m \in \mathbb{N}$ by $W_{m}(G, i)$. Let $w=e_{1} \ldots e_{m} \in W_{m}(G, i)$. Then we set

$$
F_{w}:=F_{e_{1}} \circ \cdots \circ F_{e_{m}} \text {. }
$$

We start with a variant of [91, Theorem 5.3] for GIFS.

LEMMA 6.11. - Let $G$ be a finite directed graph with set of nodes $V=\{1, \ldots, q\}$ and set of edges $E$. Let $S_{1}, \ldots, S_{q}$ be the solution of a GIFS $\left(G,\left\{F_{e}\right\}_{e \in E}\right)$ with injective contractions $F_{e}(e \in E)$, i.e.,

$$
S_{i}=\bigcup_{e: i \rightarrow j \in E_{i}} F_{e}\left(S_{j}\right) \quad(i \in V) .
$$

Here $E_{i}$ is the set of edges in $G$ starting at $i$. Assume that the collection

$$
\mathscr{C}_{i}:=\left\{F_{e}\left(S_{j}\right) ; e: i \rightarrow j \in E_{i}\right\}
$$

forms a regular chain for each $i \in V$. Then $S_{i}$ does not separate the plane for each $i \in V$. 
Proof. - Suppose that $S_{i}$ separates the plane for some $i \in V$. Since by Lemma 6.7 the set $S_{i}$ is a locally connected continuum, there exists a simple closed curve $C \subset S_{i}$ which separates the plane $(c f$. $[\mathbf{1 0 7}, \S 62, \mathrm{VI}$, Theorem 1]). Since $C$ has positive diameter, there exists a maximal $m \in \mathbb{N}$ such that $C \subset F_{w}\left(S_{j}\right)$ for some $w \in W_{m}(G, i)$. Fix such a $w$. Since $F_{w}$ is injective, we conclude that $D:=F_{w}^{-1}(C)$ is a simple closed curve satisfying

$$
D \subset S_{j}
$$

By the assumptions we know that

$$
S_{j}=\bigcup_{e \in E_{j}} F_{e}\left(S_{k}\right)
$$

where the sets in the union form a regular chain. However, by the maximality of $m$ there exist two different sets $F_{e_{1}}\left(S_{k_{1}}\right)$ and $F_{e_{2}}\left(S_{k_{2}}\right)$ in the union in (6.7) with the property that

$$
D \cap\left(F_{e_{1}}\left(S_{k_{1}}\right) \backslash F_{e_{2}}\left(S_{k_{2}}\right)\right) \neq \varnothing \quad \text { and } \quad D \cap\left(F_{e_{2}}\left(S_{k_{2}}\right) \backslash F_{e_{1}}\left(S_{k_{1}}\right)\right) \neq \varnothing .
$$

Thus, since $D$ is a simple closed curve there exist two simple arcs which are disjoint apart from their end points leading from a point in $F_{e_{1}}\left(S_{k_{1}}\right) \backslash F_{e_{2}}\left(S_{k_{2}}\right)$ to a point in $F_{e_{2}}\left(S_{k_{2}}\right) \backslash F_{e_{1}}\left(S_{k_{1}}\right)$. This contradicts the fact that $\mathscr{C}_{j}$ forms a regular chain.

Furthermore, we need the following simple result on the refinement of circular chains.

Lemma 6.12. - Let $\left\{Q_{1}, \ldots, Q_{\nu}\right\}$ be a circular chain. Suppose that $Q_{1}$ can be represented as

$$
Q_{1}=R_{1} \cup \cdots \cup R_{m},
$$

where $\left\{R_{1}, \ldots, R_{m}\right\}$ is a regular chain.

Then (possibly after reverting the order of the $R_{i}$ ) there exist $1 \leq i \leq j \leq m$ such that

$$
\left\{R_{i}, \ldots, R_{j}, Q_{2}, \ldots, Q_{\nu}\right\}
$$

forms a circular chain. Moreover, if $i>1$ then

$$
\left|R_{1} \cap\left(R_{2} \cup \cdots \cup R_{m} \cup Q_{2} \cup \cdots \cup Q_{\nu}\right)\right|=1
$$

and if $j<m$ then

$$
\left|R_{m} \cap\left(R_{1} \cup \cdots \cup R_{m-1} \cup Q_{2} \cup \cdots \cup Q_{\nu}\right)\right|=1 .
$$

Proof. - From (6.8) we conclude that

$$
\exists i, j \in\{1, \ldots, m\}: R_{i} \cap Q_{\nu} \neq \varnothing, R_{j} \cap Q_{2} \neq \varnothing .
$$

If $i$ and $j$ are not unique choose them in a way that the subchain $\left\{R_{i}, \ldots, R_{j}\right\}$ is as short as possible. Furthermore, assume that $\left\{R_{1}, \ldots, R_{m}\right\}$ is ordered in a way that $i \leq j$ holds. 
It is now a routine matter to check that (6.9) is a circular chain. Indeed, firstly, it is clear that each of the intersections $Q_{\nu} \cap R_{i}, R_{\ell} \cap R_{\ell+1}(i \leq \ell \leq j-1), R_{j} \cap Q_{2}$ and $Q_{\ell} \cap Q_{\ell+1}(2 \leq \ell \leq \nu-1)$ contain exactly one point. Furthermore, the unions $R_{k} \cap R_{\ell}$ and $Q_{k} \cap Q_{\ell}$ are empty if the indices differ by more than 1 . Since $R_{k} \subset Q_{1}$ holds for each $k \in\{1, \ldots, m\}$ we have $Q_{\ell} \cap R_{k}=\varnothing$ for $\ell \notin\{2, \nu\}$. It remains to check

$$
Q_{\nu} \cap R_{\ell}=\varnothing \quad(\ell \in\{i+1, \ldots, j\}) \quad \text { and } \quad Q_{2} \cap R_{\ell}=\varnothing \quad(\ell \in\{i, \ldots, j-1\}) .
$$

If one of these unions were nonempty, this would contradict the fact that we have chosen $i$ and $j$ in a way that the subchain $\left\{R_{i}, \ldots, R_{j}\right\}$ is as small as possible. This proves the first assertion of the lemma.

To establish the second assertion assume that $i>1$. Then, since $\left\{R_{1}, \ldots, R_{m}\right\}$ forms a regular chain, $R_{1} \cap R_{2}=\{p\}$ and $R_{1} \cap R_{\ell}=\varnothing$ for $\ell>2$. Because $R_{1} \subset Q_{1}$ we conclude that $R_{1} \cap Q_{\ell}=\varnothing$ if $\ell \in\{3, \ldots \nu-1\}$. It remains to check the intersections $R_{1} \cap Q_{2}$ and $R_{1} \cap Q_{\nu}$.

Suppose that the intersection $R_{1} \cap Q_{2}$ is nonempty. Then it can contain at most one point $q$, say. Because $R_{i} \cap Q_{2} \neq \varnothing$ for some $i>1$ and $\left|\left(R_{1} \cup R_{i}\right) \cap Q_{2}\right| \leq\left|Q_{1} \cap Q_{2}\right|=1$ we must have

$$
q \in\left(R_{1} \cap Q_{2}\right) \cap\left(R_{i} \cap Q_{2}\right) .
$$

Thus $R_{1} \cap R_{i} \neq \varnothing$ which implies that $i=2$. Hence, $q \in R_{1} \cap R_{2}$ and thus $q=p$ because this intersection contains only one point. The intersection $R_{1} \cap Q_{\nu}$ is treated likewise by observing that $j \geq i>1$. Thus $p$ is the only point contained in the intersection (6.10).

Finally, the third assertion is proved in the same way as the second one.

We now establish a criterion for the solution of a GIFS to be a simple closed curve. To this matter we will use regular and circular chains, which were already used in $[\mathbf{1 2 2}, \mathbf{1 2 4}]$. Moreover, we will use the following results from topology.

Lemma 6.13 (Janiszewski's first theorem, see $[\mathbf{1 0 7}, \S 61, \mathrm{I}$, Theorem 7]). - Suppose that $M_{1}$ and $M_{2}$ are two compact subsets of $\mathbb{R}^{2}$ with $\left|M_{1} \cap M_{2}\right|=1$. If $M_{1} \cup M_{2}$ separates the plane then the same is true for $M_{1}$ or for $M_{2}$.

Note that $\mathbb{S}^{2}$ is a Janiszewski Space (see $[\mathbf{1 0 7}, \S 61$, I, Theorem 2]). Thus the above lemma follows from $\left[\mathbf{1 0 7}, \S 61, \mathrm{I}\right.$, Theorem 7] if one replaces the space $\mathbb{R}^{2}$ by $\mathbb{S}^{2}$. Since the sets $M_{1}$ and $M_{2}$ are compact, the lemma is also true for the space $\mathbb{R}^{2}$.

Lemma 6.14 (cf. $\left[\mathbf{1 0 7}, \S 52, \mathrm{IV}\right.$, Theorem 1]). - If $X \subset \mathbb{R}^{2}$ is a locally connected continuum containing no separating point and no $\theta$-curve, then $X$ is a simple closed curve (unless it is a single point). 
6.5.3. A sufficient condition for the subtiles $\mathcal{T}(i)(i \in \mathscr{Q})$ to be disklike. We first give some preliminaries on GIFS. We start with the following result.

LEMMA 6.15. - Let $T \subset \mathbb{R}^{2}$ be a set having the following properties.

- $T$ is the closure of its interior.

- $\partial T=S_{1} \cup \cdots \cup S_{q}$ with $^{(1)}\left|S_{j}\right| \geq 2$ for $1 \leq j \leq q$. Here $S_{1}, \ldots, S_{Q}(Q \geq q)$ are the solution of a GIFS with injective contractions directed by a finite directed graph $G=G(V, E)$ with $V=\{1, \ldots, Q\}$.

- $\left\{S_{1}, \ldots, S_{q}\right\}$ is a circular chain.

- The elements of the union

$$
S_{i}=\bigcup_{e: i \rightarrow j} F_{e}\left(S_{j}\right) \quad(1 \leq i \leq Q)
$$

form a regular chain.

Then

$$
K_{m_{1}, \ldots, m_{q}}:=\left\{F_{w}\left(S_{i}\right) ; i \in V, w \in W_{m_{i}}(G, i)\right\}
$$

is a circular chain.

REMARK 6.16. - Note that iteration of the GIFS equation for $S_{1}, \ldots, S_{q}$ yields

$$
\partial T:=\bigcup_{Z \in K_{m_{1}, \ldots, m_{q}}} Z .
$$

Proof. - The proof is done by induction on $m:=\sum_{i=1}^{q} m_{i}$. Let

$$
\mathcal{K}_{m}:=\left\{K_{m_{1}, \ldots, m_{q}} ; \sum_{i=1}^{q} m_{i}=m\right\} .
$$

We want to prove that each element of $\mathcal{K}_{m}(m \in \mathbb{N})$ forms a circular chain. The induction start is simple as the assertion is true for the only element $\left\{S_{1}, \ldots, S_{q}\right\}$ of $\mathcal{K}_{0}$ by assumption.

To perform the induction step assume from now that each element of $\mathcal{K}_{m}$ is a circular chain. We need to show that the same is true for each element of $\mathcal{K}_{m+1}$. Note that each element $K \in \mathcal{K}_{m+1}$ emerges from a circular chain $K^{\prime} \in \mathcal{K}_{m}$ in the following way. For a given fixed $i \in V$ replace each element

$$
F_{w}\left(S_{i}\right) \in K^{\prime}
$$

by the elements of the collection

$$
\left\{F_{w o e}\left(S_{j}\right) ; e: i \rightarrow j \text { is an edge in } G(V, E)\right\} .
$$

If we replace the elements of the form (6.12) step by step we get finitely many collections $C_{0}, \ldots, C_{L}$ with $C_{0}=K^{\prime}$ and $C_{L}=K$. Thus the induction step is proved

(1) By arguing in the same way as in the proof of Lemma 5.8 we see that this causes no loss of generality. Indeed, if we have a representation $\partial T=S_{1} \cup \cdots \cup S_{q}$ we can remove all sets $S_{j}$ with $\left|S_{j}\right|=1$ without changing the union, i.e., the union of the remaining sets is still equal to $\partial T$. 
if we can show that $C_{\ell}$ is a circular chain provided that $C_{\ell-1}$ is a circular chain $(1 \leq \ell \leq L)$. To this matter let $\ell \in\{1, \ldots, L\}$ be given and assume that $C_{\ell-1}$ is a circular chain. Observe that $C_{\ell}$ emerges from $C_{\ell-1}$ by replacing a set of the shape (6.12) by the elements of the collection (6.13). Thus in view of Lemma 6.12 there are two possibilities for $C_{\ell}$.

(i) $C_{\ell}$ is a circular chain. In this case we are ready.

(ii) There is a set $X:=F_{w \circ e_{0}}\left(S_{j_{0}}\right)$ in the collection (6.13) such that, setting $Y:=$ $\bigcup_{Z \in C_{\ell} \backslash\{X\}} Z$ we have

$$
\partial T=X \cup Y
$$

with $X \cap Y=\{p\}$, a single point. Let $x \in X \backslash Y$ (such an element $x$ exists because $|X| \geq 2$ ). Since $Y$ is a compact set there exists $\varepsilon>0$ such that

$$
B_{\varepsilon}(x) \cap Y=\varnothing .
$$

Because $T$ is the closure of its interior, $x \in \partial T$ implies the existence of $x_{1}, x_{2} \in$ $B_{\varepsilon}(x)$ with $x_{1} \in \operatorname{int}(T)$ and $x_{2} \in \mathbb{R}^{2} \backslash T$. Thus $\partial T$ separates $x_{1}$ and $x_{2}$. Lemma 6.13 now implies that $X$ or $Y$ separates $x_{1}$ and $x_{2}$. From (6.14) we immediately see that $X$ separates $x_{1}$ and $x_{2}$. However, on the other hand, $X \subset S_{i}$ holds for some $i \in V$ and as the union in (6.11) forms a regular chain, Lemma 6.11 implies that $S_{i}$ does not separate the plane. Thus also $X$ does not separate the plane, a contradiction.

So only case (i) can occur and, hence, $C_{\ell}$ is a circular chain. Since $\ell$ was arbitrary this yields that each collection in $\mathcal{K}_{m+1}$ is a circular chain. This concludes the induction proof.

The following proposition is of interest in its own right. It provides a criterion for the homeomorphy to a disk that is usable for large classes of IFS tiles as well as GIFS tiles. We will use it to derive a criterion for the homeomorphy to a disk of the subtiles $\mathcal{J}(i)(i \in \mathscr{C})$ which can be checked algorithmically by using the graphs we introduced in the previous chapter.

Proposition 6.17. - Let $T \subset \mathbb{R}^{2}$ be a set having the following properties.

(i) $T$ is the closure of its interior.

(ii) $\partial T=S_{1} \cup \cdots \cup S_{q}$ with $^{(2)}\left|S_{j}\right| \geq 2$ for $1 \leq j \leq q$. Here $S_{1}, \ldots, S_{Q}(Q \geq q)$ are the solution of a GIFS with injective contractions directed by a graph $G(V, E)$ with $V=\{1, \ldots, Q\}$.

(iii) $\left\{S_{1}, \ldots, S_{q}\right\}$ is a circular chain.

(iv) The elements of the union

$$
S_{i}=\bigcup_{e: i \rightarrow j} F_{e}\left(S_{j}\right) \quad(1 \leq i \leq Q)
$$

form a regular chain.

Then $\partial T$ is a simple closed curve and $T$ is homeomorphic to a closed disk.

$\overline{(2) \text { see Footnote } 1}$ 
Proof. - We prove the result in three steps.

(a) $S_{i}$ is a locally connected continuum for each $i \in V$. This is a consequence of Lemma 6.7.

(b) $\partial T=S_{1} \cup \cdots \cup S_{q}$ contains no separating point. To see this assume on the contrary that there exist $p_{1}, p_{2} \in \partial T$ which are separated by $s \in \partial T$. According to Lemma 6.15 there is a circular chain $K \in \mathcal{K}_{\nu}$ such that

$$
\operatorname{diam} C<\varepsilon
$$

holds for each $C \in K$ with

$$
\varepsilon<\frac{1}{2} \min \left(\left|p_{1}-p_{2}\right|,\left|p_{1}-s\right|,\left|p_{2}-s\right|\right) .
$$

Thus there exist different sets $C_{p_{1}}, C_{p_{2}}, C_{s} \in K$ with

$$
p_{1} \in C_{p_{1}} \backslash\left(C_{p_{2}} \cup C_{s}\right), \quad p_{2} \in C_{p_{2}} \backslash\left(C_{p_{1}} \cup C_{s}\right), \quad s \in C_{s} \backslash\left(C_{p_{1}} \cup C_{p_{2}}\right) .
$$

Since $K$ is a circular chain, $C_{p_{1}}$ and $C_{p_{2}}$ can be connected by elements of $K \backslash$ $\left\{C_{s}\right\}$. Because all elements of $K$ are locally connected continua this implies that $p$ and $q$ can be connected by an arc avoiding $C_{s}$ and, hence, avoiding $s$, a contradiction.

(c) $\partial T$ contains no $\theta$-curve. Assume on the contrary that $\partial T$ contains a $\theta$-curve. Let $a_{1}, a_{2}, a_{3}$ be the simple arcs that form the $\theta$-curve and let $p_{i} \in \operatorname{int} a_{i}$ and $s \in a_{1} \cap a_{2} \cap a_{3}$. Choose

$$
\varepsilon<\frac{1}{2} \min _{i \neq j}\left(\min _{r \in a_{j}}\left|p_{i}-r\right|\right) .
$$

By Lemma 6.15 there is a circular chain $K \in \mathcal{K}_{\nu}$ with

$$
\operatorname{diam} C<\varepsilon \quad \text { for all } C \in K \text {. }
$$

Choose $C_{1}, C_{2}, C_{3}, D \in K$ such that $p_{i} \in C_{i}$ and $s \in D$ (by the choice of $\varepsilon$ the sets $C_{1}, C_{2}, C_{3}, D$ have pairwise empty intersection). Since $K$ is a circular chain there exist the regular subchains

$$
\begin{aligned}
& D \leftrightarrow E_{1} \leftrightarrow E_{2} \leftrightarrow \cdots \leftrightarrow E_{\ell_{1}}=C_{1}, \\
& D \leftrightarrow F_{1} \leftrightarrow F_{2} \leftrightarrow \cdots \leftrightarrow F_{\ell_{2}}=C_{2}, \\
& D \leftrightarrow G_{1} \leftrightarrow G_{2} \leftrightarrow \cdots \leftrightarrow G_{\ell_{3}}=C_{3} .
\end{aligned}
$$

Suppose first that $E_{1}, F_{1}, G_{1}$ are pairwise distinct elements of $K$. Then $D$ has nonempty intersection with each of the sets $E_{1}, F_{1}, G_{1}$ which is impossible for the elements of a circular chain. If these elements are not pairwise distinct elements of $K$ we may assume w.l.o.g. that $E_{1}=F_{1}$. According to the choice of $\varepsilon$ there is a maximal $j<\min \left(\ell_{1}, \ell_{2}\right)$ such that $E_{j}=F_{j}$. For this $j$ we have (setting $E_{0}:=D$ if necessary)

$$
E_{j-1} \cap E_{j} \neq \varnothing, \quad E_{j+1} \cap E_{j} \neq \varnothing, \quad F_{j+1} \cap E_{j} \neq \varnothing .
$$


By the maximality of $j$ this means that $E_{j}$ intersects three distinct elements of $K$, a contradiction to the fact that $K$ is a circular chain. Thus $\partial T$ contains no $\theta$-curve.

By (a), (b) and (c) we conclude from Lemma 6.14 that $\partial T$ is a simple closed curve. The fact that $T$ is homeomorphic to a closed disk now follows from the Jordan Curve Theorem ( $c f$. [107, $\S 61$, II, Theorem 1]).

From this result we deduce the following algorithmic criterion for the subtiles $\mathcal{T}(i)$ to be homeomorphic to a closed disk.

THEOREM 6.18. - Let $\sigma$ be a primitive unit Pisot substitution whose dominant eigenvalue has degree 3 that has the tiling property. Then each of the subtiles $\mathcal{J}(i)(i \in \mathscr{G})$ is homeomorphic to a closed disk if the following conditions are satisfied.

(1) The boundary-connectivity graph of $\mathcal{J}(i)$ is either a single node or a circle for each $i \in \mathscr{C}$.

(2) For each $i \in \mathscr{G}$ each edge of the boundary-connectivity graph of $\mathcal{T}(i)$ corresponds to an intersection containing exactly one point.

(3) For each node $[i, \gamma, j]$ of $\mathscr{G}_{\mathrm{srs}}^{(B)}$, the boundary-refinement-connectivity graph of $B[i, \gamma, j]$ is a line (that may be empty or degenerate to a single node).

(4) For each node $[i, \gamma, j]$ of $\mathscr{G}_{\mathrm{srs}}^{(B)}$ each edge of the boundary-refinement-connectivity graph of $B[i, \gamma, j]$ corresponds to an intersection containing exactly one point.

REMARK 6.19. - If we consider also the case contained in Remark 4.14 one could show with some effort that the above criterion is necessary and sufficient for the subtiles to be homeomorphic to a closed disk.

Proof. - Let $i \in \mathscr{G}$ be arbitrary but fixed. By Theorem 5.7 the boundary $\partial \mathcal{J}(i)$ is the union of sets $\mathcal{J}(i) \cap \mathcal{J}(j)+\gamma$ where $[i, \gamma, j]$ is a node of the SR-boundary graph $\mathscr{G}_{\mathrm{srs}}^{(B)}$. Lemma 5.8 implies that we can remove each intersection which is a single point from this union. Denote the collection of the remaining intersections by $\left\{S_{1}, \ldots, S_{q}\right\}$. Then $\partial \mathcal{J}(i)=S_{1} \cup \cdots \cup S_{q}$ satisfies Conditions (i) and (ii) of Proposition 6.17. Conditions (1) and (2) of the theorem imply that the collection $\left\{S_{1}, \ldots, S_{q}\right\}$ forms a circular chain, thus also Condition (iii) of Proposition 6.17 is satisfied. Condition (iv) of this proposition follows from Conditions (3) and (4) of the theorem. Since $\mathbb{H}_{c} \cong \mathbb{R}^{2}$ the theorem is now established by applying Proposition 6.17.

It remains to explain how to check the conditions of Theorem 6.18 algorithmically using the graphs we have at our disposal.

Checking (1) and (3) : This corresponds to building the boundary-connectivity graph and the boundary-refinement-connectivity graph. 
Checking (2) and (4) : We discuss how to check (2). Checking (4) runs along the same lines. Consider an edge of the boundary-connectivity graph. This edge corresponds to an intersection of three subtiles $\mathcal{J}(i), \mathcal{T}\left(j_{1}\right)+\gamma_{1}, \mathcal{J}\left(j_{2}\right)+\gamma_{2}$ of the self-replicating tiling. Thus the points in this intersection are in one-to-one correspondence with the essentially different infinite walks leading away from the node $\left[i, \gamma_{1}, j_{1}, \gamma_{2}, j_{2}\right]$ of the triple point graph (see Theorem 5.25). If there is only one walk leading away from this node, we are finished, if there exist more, we have to check whether they are essentially different. This can be done algorithmically by Proposition 5.33.

\subsection{The fundamental group}

In this section we want to establish criteria that allow to conclude that a given subtile (or central tile) has nontrivial or even uncountable fundamental group $\pi_{1}$. The proof of these criteria relies on the following topological lemmas.

LEMma 6.20 ([123, Lemma 6.1]). - Let $B_{0}, B_{1}, B_{2} \subset \mathbb{R}^{2}$ be locally connected continua with the following properties.

(i) $\operatorname{int}\left(B_{i}\right) \cap \operatorname{int}\left(B_{j}\right)=\varnothing$ for $i \neq j$.

(ii) $B_{i}$ is the closure of its interior $(0 \leq i \leq 2)$.

(iii) $\mathbb{S}^{2} \backslash \operatorname{int}\left(B_{i}\right)$ is a locally connected continuum $(0 \leq i \leq 2)$.

(iv) There exist $x_{1}, x_{2} \in B_{0} \cap B_{1} \cap B_{2}$ with $x_{1} \neq x_{2}$ and $x_{1} \in \operatorname{int}\left(B_{0} \cup B_{1} \cup B_{2}\right)$.

Then there exists $i \in\{0,1,2\}$ such that $B_{i} \cup B_{i+1}$ has a bounded complementary component $U$ with $U \cap \operatorname{int}\left(B_{i+2}\right) \neq \varnothing$ (the indices are to be taken modulo 3$)$.

Lemma 6.21 ([123, Proposition 4.1]). - Let $K \subset \mathbb{S}^{2}$ be a locally arcwise connected set. If $\mathbb{S}^{2} \backslash K$ is disconnected then $K$ contains a nontrivial loop.

Lemma 6.22 ([123, Proposition 4.2]; see also [65]). - Let $K \subset \mathbb{S}^{2}$ be a locally arcwise connected continuum. Suppose that $\mathbb{S}^{2} \backslash K$ has infinitely many components. Then the following assertions hold.

(i) $\pi_{1}(K)$ is not free.

(ii) $\pi_{1}(K)$ is uncountable.

(iii) $K$ is not locally simply connected.

(iv) $K$ has no universal cover.

We now state the first result on the fundamental group of a subtile. After that we discuss how it can be checked by using the graphs we defined in Chapter 5 .

TheOREM 6.23. - Suppose that $\sigma$ is a primitive unit Pisot substitution whose dominant eigenvalue has degree 3 that has the tiling property. Suppose that $\mathcal{T}(j)$ is connected for each $j \in \mathscr{G}$. Then the subtile $\mathcal{T}(i)(i \in \mathscr{G})$ has nontrivial fundamental group if there exist $\left[\gamma_{1}, i_{1}\right],\left[\gamma_{2}, i_{2}\right] \in \Gamma_{\mathrm{srs}}$ such that $[\mathbf{0}, i],\left[\gamma_{1}, i_{1}\right],\left[\gamma_{2}, i_{2}\right]$ are pairwise disjoint and the following conditions are satisfied. 
(1) The intersection $\mathcal{T}(i) \cap\left(\mathcal{T}\left(i_{1}\right)+\gamma_{1}\right) \cap\left(\mathcal{T}\left(i_{2}\right)+\gamma_{2}\right)$ contains at least two elements $\mathbf{x}_{1}, \mathbf{x}_{2}$. The point $\mathbf{x}_{1}$ is contained in $\operatorname{int}\left(\mathcal{J}(i) \cup\left(\mathcal{J}\left(i_{1}\right)+\gamma_{1}\right) \cup\left(\mathcal{J}\left(i_{2}\right)+\gamma_{2}\right)\right)$.

(2) Let $\left[\gamma_{0}, i_{0}\right]:=[\mathbf{0}, i]$ for convenience. Then there exists $N \in \mathbb{N}$ such that for all $\ell \in\{0,1,2\}$ one can find $\delta_{\ell} \in \mathbb{H}_{c}$ such that $\left[\gamma_{\ell}+\delta_{\ell}, i_{\ell}\right] \in \Gamma_{\mathrm{srs}}$ and (indices are to be taken $\bmod 3$ )

$\left[\gamma_{\ell}+\delta_{\ell}, i_{\ell}\right] \notin \mathbf{E}_{1}^{* N}[\mathbf{0}, i], \quad\left[\gamma_{\ell+1}+\delta_{\ell}, i_{\ell+1}\right] \in \mathbf{E}_{1}^{* N}[\mathbf{0}, i], \quad\left[\gamma_{\ell+2}+\delta_{\ell}, i_{\ell+2}\right] \in \mathbf{E}_{1}^{* N}[\mathbf{0}, i]$.

Proof. (see [123, Proposition 6.2] for an analog for self-affine lattice tiles)

We first prove that $B_{0}:=\mathcal{J}(i), B_{1}:=\mathcal{J}\left(i_{1}\right)+\gamma_{1}$ and $B_{2}:=\mathcal{J}\left(i_{2}\right)+\gamma_{2}$ satisfy the conditions of Lemma 6.20. Indeed all are locally connected continua by Theorem 4.9 (connectivity of each $\mathcal{T}(i)$ implies the local connectivity of each $\mathcal{T}(i)$ ).

Condition (i) of Lemma 6.20 is true because we assume the tiling property in the statement of the theorem and Condition (ii) follows because each tile $\mathcal{J}(j), j \in \mathscr{G}$, as well as each of their translates is the closure of its interior by Theorem 2.6. Condition (iii) is true because the closed connected set $\mathbb{R}^{2} \backslash \operatorname{int}(\mathcal{J}(j))$ can be written as a locally finite union of translates of the subtiles $\mathcal{T}(1), \ldots, \mathcal{T}(n)$ by the tiling property. Thus it is also locally connected and its closure in $\mathbb{S}^{2}$, the set $\mathbb{S}^{2} \backslash \operatorname{int}(\mathcal{T}(j))$, is a locally connected continuum. The same holds for each translate of $\mathcal{T}(j)$. Condition (iv) of Lemma 6.20 is just the same as condition (1) of the theorem.

Thus we may apply Lemma 6.20 and therefore assume that (recall that $[0, i]=$ $\left.\left[\gamma_{0}, j_{0}\right]\right)$ for some $\ell \in\{0,1,2\}$ there is a point $\mathbf{z} \in \operatorname{int}\left(\mathcal{J}\left(i_{\ell}\right)+\gamma_{\ell}\right)$ which is contained in a bounded complementary component $U$ of $\left(\mathcal{T}\left(i_{\ell+1}\right)+\gamma_{\ell+1}\right) \cup\left(\mathcal{T}\left(i_{\ell+2}\right)+\gamma_{\ell+2}\right)$. Fix this $\ell$ from now. In view of condition (2) we know that $\mathcal{T}\left(i_{\ell}\right)+\gamma_{\ell}+\delta_{\ell}$ is a piece of the self-replicating tiling but it is not a piece of $\mathbf{h}^{-N} \mathcal{J}(i)$ since by (3.4) we have

$$
\mathbf{h}^{-N} \mathcal{J}(i)=\bigcup_{[\gamma, k] \in \mathbf{E}_{1}^{* N}[\mathbf{0}, i]}(\mathcal{T}(k)+\gamma) \text {. }
$$

By the tiling property, we deduce that int $\left(\mathcal{T}\left(i_{\ell}\right)+\gamma_{\ell}+\delta_{\ell}\right)$ has empty intersection with $\mathbf{h}^{-N} \mathcal{J}(i)$. This means that $\mathbf{z}+\delta_{\ell}$ is contained in a complementary component $U_{0}$ of $\mathbf{h}^{-N} \mathcal{J}(i)$.

But since condition (2) also implies that $\left(\mathcal{J}\left(i_{\ell+1}\right)+\delta_{\ell}+\gamma_{\ell+1}\right) \cup\left(\mathcal{T}\left(i_{\ell+2}\right)+\delta_{\ell}+\right.$ $\left.\gamma_{\ell+2}\right) \subset \mathbf{h}^{-N} \mathcal{T}(i)$ we deduce that $U_{0} \subset U$. Since $U$ is bounded, also $U_{0}$ is bounded.

Thus $\mathbf{h}^{-N} \mathcal{T}(i)$ and therefore also $\mathcal{T}(i)$ has at least one bounded complementary component. The result now follows from Lemma 6.21 .

It remains to explain how to check the conditions of Theorem 6.23 algorithmically using the graphs we have at our disposal.

Checking (1) algorithmically : The existence of at least two points $\mathbf{x}_{1}, \mathbf{x}_{2} \in \mathcal{J}(i) \cap$ $\left(\mathcal{J}\left(i_{1}\right)+\gamma_{1}\right) \cap\left(\mathcal{J}\left(i_{2}\right)+\gamma_{2}\right)$ can be checked with help of the triple point graph by using Theorem 5.25 and Proposition 5.33.

The fact that the point $\mathbf{x}_{1}$ is contained in $\operatorname{int}\left(\mathcal{J}(i) \cup\left(\mathcal{T}\left(i_{1}\right)+\gamma_{1}\right) \cup\left(\mathcal{T}\left(i_{2}\right)+\gamma_{2}\right)\right)$ means that $\mathbf{x}$ is a triple point but not a quadruple point. Thus in view of 
Theorems 5.25 and 5.30 such a point exists if a certain infinite walk which exists in the triple point graph does not exist in the quadruple point graph.

Checking (2) : This is done by scrutinizing $\mathbf{E}_{1}^{* N}[0, i]$ for increasing $N$. More precisely, we consider $[\mathbf{0}, i],\left[\gamma_{1}, i_{1}\right],\left[\gamma_{2}, i_{2}\right]$ that satisfy (1). We fix $N$. For every $\delta$ whose modulus is bounded by the sum of the maximal modulus of a vector in $\mathbf{E}_{1}^{* N}[\mathbf{0}, i](\ell=1,2,3)$ and the maximal modulus of the vectors $\gamma_{\ell}$, we translate the three faces $\left[\gamma_{\ell}, i_{\ell}\right](0 \leq \ell \leq 2)$ by $\delta$. If for each $\ell \in\{0,1,2\}$ we find a translate $\delta=\delta_{\ell}$ satisfying condition (2), we are done. If not, it may help to repeat the procedure for a different choice of $N$. We will illustrate this procedure in two examples (see Examples 6.24 and 6.25 below).

EXAMPLE 6.24. - We consider the substitution $\sigma_{5}(1)=123, \sigma_{5}(2)=1, \sigma_{5}(3)=31$. Our goal is to show that $\mathcal{J}(2)$ has nontrivial fundamental group for this example. To this matter we will prove Conditions (1) and (2) of Theorem 6.23 for the elements $[\mathbf{0}, 2],[\mathbf{0}, 3],[\pi(1,0,-1), 1]$ of $\Gamma_{\text {srs }}$.

Checking Condition (1) : First observe that $[2, \mathbf{0}, 3, \pi(1,0,-1), 1]$ is a node of the triple point graph from which there start infinitely many infinite walks (this can be checked easily by looking at the loop structure of the triple point graph in Figure $\left.6.2^{(3)}\right)$. Thus the intersection

$$
\mathcal{J}(2) \cap \mathcal{J}(3) \cap(\mathcal{J}(1)+\pi(1,0,-1))
$$

contains infinitely many points by Proposition 5.33. Since the quadruple point graph contains only finitely many infinite walks (see Figure 6.3) there is certainly a walk in the triple point graph starting at $[2, \mathbf{0}, 3, \pi(1,0,-1), 1]$ that does not occur in the quadruple point graph. This shows that Condition (1) holds.

Checking Condition (2) : Take $N=4, \delta_{0}=\pi(1,0,-1), \delta_{1}=\pi(2,1,-3)$ and $\delta_{2}=$ $\pi(2,0,-2)$. First, by iterating $\mathbf{E}_{1}^{*}$ four times for the argument $[\mathbf{0}, 2]$ we obtain

$$
\begin{aligned}
\left(\mathbf{E}_{1}^{*}\right)^{4}[\mathbf{0}, 2]=\{ & {[\pi(2,1,-3), 1],[\pi(2,1,-3), 2],[\pi(2,0,-2), 1],[\pi(2,0,-2), 2], } \\
& {[\pi(2,0,-2), 3],[\pi(2,2,-3), 1],[\pi(2,1,-2), 1],[\pi(1,0,-1), 3], } \\
& {[\pi(3,1,-4), 1],[\pi(3,1,-4), 2],[\pi(3,0,-3), 3],[\pi(2,-1,-1), 3], } \\
& {[\pi(2,-1,-1), 1],[\pi(1,-2,0), 3]\} . }
\end{aligned}
$$

Looking at this list of values we notice that

$$
\begin{array}{lll}
{[\pi(1,0,-1), 2] \notin\left(\mathbf{E}_{1}^{*}\right)^{4}[\mathbf{0}, 2],} & {[\pi(1,0,-1), 3],[\pi(2,0,-2), 1] \in\left(\mathbf{E}_{1}^{*}\right)^{4}[\mathbf{0}, 2],} \\
{[\pi(2,1,-3), 3] \notin\left(\mathbf{E}_{1}^{*}\right)^{4}[\mathbf{0}, 2],} & {[\pi(2,1,-3), 1],[\pi(3,1,-4), 1] \in\left(\mathbf{E}_{1}^{*}\right)^{4}[\mathbf{0}, 2],} \\
{[\pi(3,0,-3), 1] \notin\left(\mathbf{E}_{1}^{*}\right)^{4}[\mathbf{0}, 2],} & {[\pi(2,0,-2), 2],[\pi(2,0,-2), 3] \in\left(\mathbf{E}_{1}^{*}\right)^{4}[\mathbf{0}, 2] .}
\end{array}
$$

Checking the conditions in the definition of $\Gamma_{\text {srs }}$ in (3.1) for all occurring vectors we see that they all belong to $\Gamma_{\text {srs }}$. Thus Condition (2) is satisfied.

(3) We omit the projection " $\pi$ " in the caption of the nodes of this and all the other figures of this chapter in order to keep the size of the nodes smaller. 

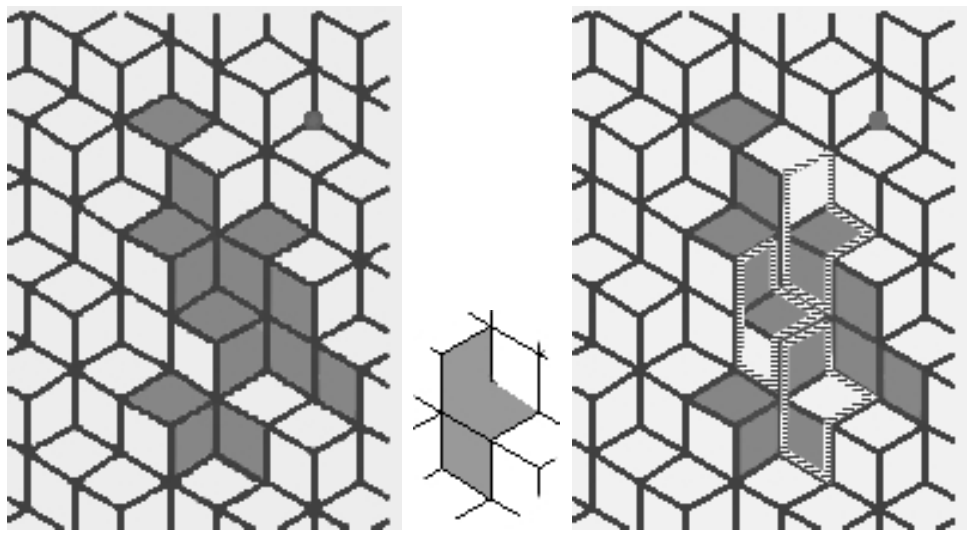

Figure 6.1. Consider the substitution $\sigma_{5}(1)=123, \sigma_{5}(2)=1, \sigma_{5}(3)=31$. The left figure contains a geometric representation of $\left(\mathbf{E}_{1}^{*}\right)^{4}[\mathbf{0}, 2]$ in the selfreplicating translation set $\Gamma_{\text {srs }}$. Each piece $[\mathbf{x}, i]$ is represented as a rhombus in the plane; the shape of the rhombus depends of the letter used. In the right figure, we see that the patch $[\mathbf{0}, 2],[\mathbf{0}, 3],[\pi(1,0,-1), 1]$ (depicted in the middle) can be translated such that two pieces of the shape belong to $\left(\mathbf{E}_{1}^{*}\right)^{4}[\mathbf{0}, 2]$ and the third belongs to $\Gamma_{\mathrm{srs}} \backslash\left(\mathbf{E}_{1}^{*}\right)^{4}[\mathbf{0}, 2]$. This proves the second condition of Theorem 6.23.

Now we may apply Theorem 6.23 , in order to conclude that the subtile $\mathcal{T}(2)$ has nontrivial fundamental group.

Notice that we can check the condition on $\left(\mathbf{E}_{1}^{*}\right)^{4}$ geometrically as follows. Each piece $[\mathbf{x}, j]$ can represented by a face. We have to translate the patch $\left\{[\mathbf{0}, i],\left[\gamma_{1}, j_{1}\right],\left[\gamma_{2}, j_{2}\right]\right\}$ at three different places of $\Gamma_{\text {srs }}$ such that exactly two pieces of the translated patch coincide with elements of $\left(\mathbf{E}_{1}^{*}\right)^{4}[\mathbf{0}, 2]$ at each place. The piece having empty intersection with $\left(\mathbf{E}_{1}^{*}\right)^{4}[\mathbf{0}, 2]$ has to be a translate of a different element of the patch at each of the places. An illustration of this procedure is depicted in Figure 6.1.

EXAMPLE 6.25. - In this example we outline how to prove that the subtile $\mathcal{J}(1)$ of the substitution $\sigma_{6}$ has nontrivial fundamental group. Indeed, the substitution $\sigma_{6}$ satisfies the conditions of Theorem 6.23 for $i=1$. This can be seen in the same way as in Example 6.24. Since the node $[2, \pi(1,-1,0), 3, \pi(1,0,-1), 1]$ is the starting point of infinitely many infinite walks in the triple point graph, and the quadruple point graph contains only finitely many infinite walks (see Figures 6.6 and 6.7), Condition (1) of Theorem 6.23 holds. Moreover, considering the elements $[\mathbf{0}, 2],[\pi(1,-1,0), 3]$ and $[\pi(1,0,-1), 1]$ of $\Gamma_{\mathrm{srs}}$, the 9 -th iteration $\left(\mathbf{E}_{1}^{*}\right)^{9}[\mathbf{0}, 2]$ and the translation vectors $\delta_{0}=$ $\pi(6,-9,3), \delta_{1}=\pi(4,-7,4)$ and $\delta_{2}=\pi(4,-8,5)$ can be used to get Condition (2). See Example 6.28 for additional details. 


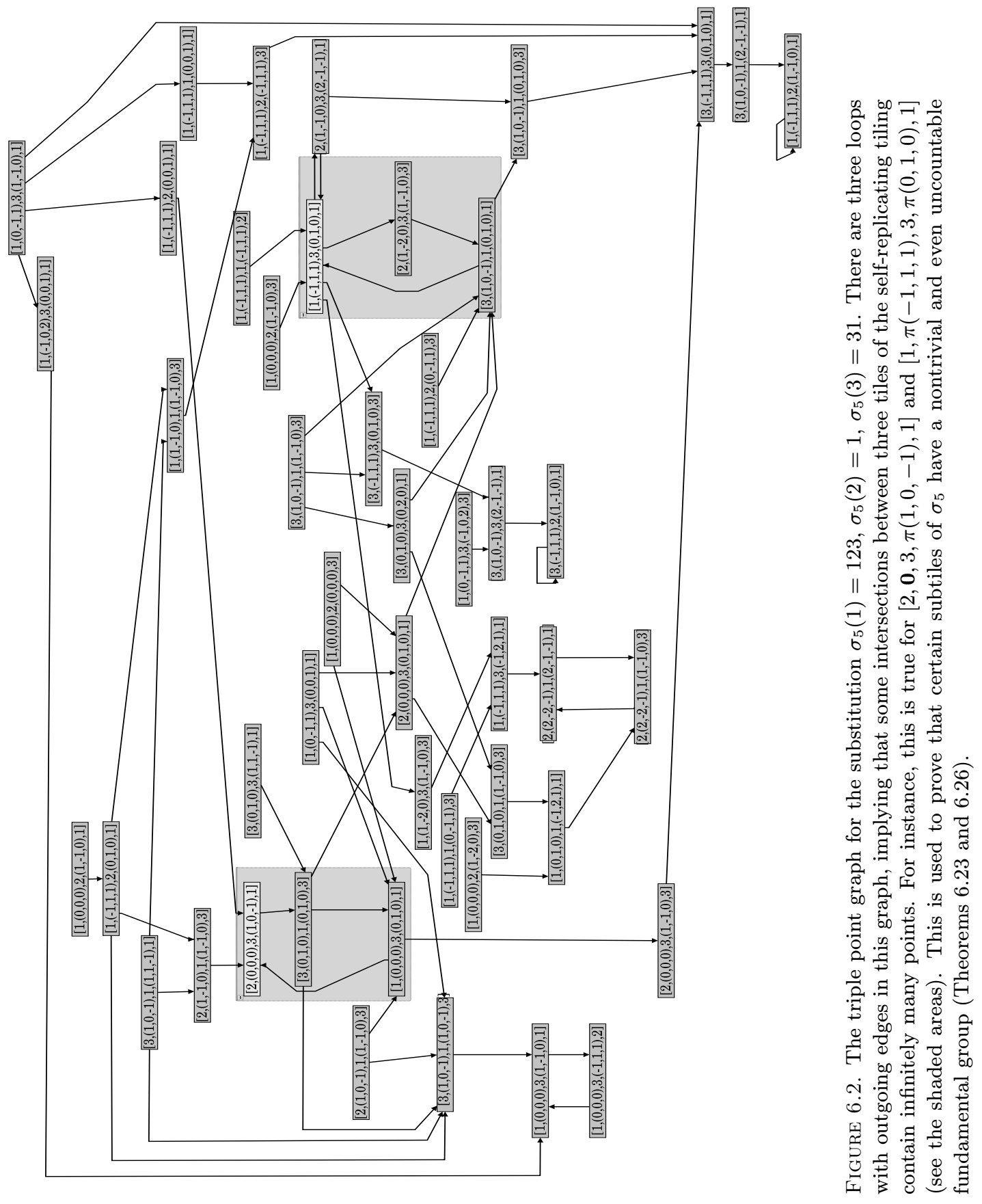



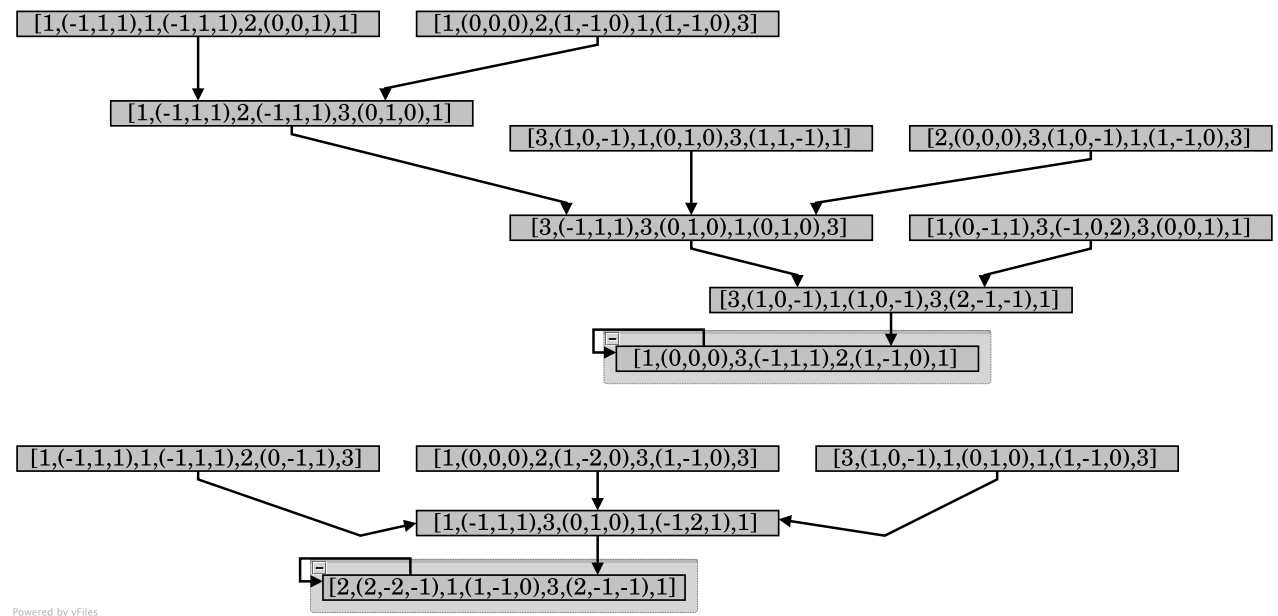

Figure 6.3. The quadruple point graph for the substitution $\sigma_{5}(1)=123$, $\sigma_{5}(2)=1, \sigma_{5}(3)=31$. Each node of this graph has a single outgoing edge. Therefore, each nonempty intersection between four tiles of the selfreplicating tiling is a single point. This information is needed in order to apply Theorems 6.23 and 6.26 .

The following theorem allows to get even more information on the fundamental group of the subtiles $\mathcal{T}(i)(i \in \mathscr{G})$

THEOREM 6.26. - Let the same setting as in Theorem 6.23 be in force but assume that the following conditions hold.

1. The intersection $\mathcal{J}(i) \cap\left(\mathcal{T}\left(i_{1}\right)+\gamma_{1}\right) \cap\left(\mathcal{J}\left(i_{2}\right)+\gamma_{2}\right)$ contains at least two elements $\mathbf{x}_{1}, \mathbf{x}_{2}$. The point $\mathbf{x}_{1}$ is contained in $\operatorname{int}\left(\mathcal{J}(i) \cup\left(\mathcal{J}\left(i_{1}\right)+\gamma_{1}\right) \cup\left(\mathcal{J}\left(i_{2}\right)+\gamma_{2}\right)\right)$.

2. Let $\left[\gamma_{0}, i_{0}\right]:=[\mathbf{0}, i]$ for convenience. Then there exists a positive integer $N$ such that for all $\ell \in\{0,1,2\}$ one can find $\delta_{\ell} \in \mathbb{Z}^{d}$ and $\left[\kappa_{\ell}, j_{\ell}\right] \in \Gamma_{\text {srs }}$ with $\left[\kappa_{\ell}, j_{\ell}\right] \neq[\mathbf{0}, i]$ such that (indices are to be taken $\bmod 3$ )

(i) $\left[i, \kappa_{\ell}, j_{\ell}\right]$ is a node of the $S R$-boundary graph;

(ii) $\left[\gamma_{\ell}+\delta_{\ell}, i_{\ell}\right] \in \mathbf{E}_{1}^{* N}\left[\kappa_{\ell}, j_{\ell}\right],\left[\gamma_{\ell+1}+\delta_{\ell}, i_{\ell+1}\right] \in \mathbf{E}_{1}^{* N}[\mathbf{0}, i],\left[\gamma_{\ell+2}+\delta_{\ell}, i_{\ell+2}\right] \in$ $\mathbf{E}_{1}^{* N}[\mathbf{0}, i]$.

(iii) For each $\ell \in\{0,1,2\}$, there exists a node in the SR-boundary graph of the shape $\left[i, \overline{\kappa_{\ell}}, \overline{j_{\ell}}\right]$ such that an infinite number of infinite walks with an even number of edges of type 2 lead from $\left[i, \overline{\kappa_{\ell}}, \overline{j_{\ell}}\right]$ to $\left[i, \kappa_{\ell}, j_{\ell}\right]$.

Then the fundamental group $\pi_{1}(\mathcal{J}(i))$ is uncountable and not free. Moreover, $\mathcal{J}(i)$ is not locally simply connected and admits no universal cover.

Proof. - Since $\left[\kappa_{\ell}, j_{\ell}\right] \in \Gamma_{\text {srs }}$ and $\left[\kappa_{\ell}, j_{\ell}\right] \neq[\mathbf{0}, i]$, the sets $\mathbf{E}_{1}^{* N}\left[\kappa_{\ell}, j_{\ell}\right]$ and $\mathbf{E}_{1}^{* N}[\mathbf{0}, i]$ are disjoint (see Proposition 3.5). From $\left[\gamma_{\ell}+\delta_{\ell}, i_{\ell}\right] \in \mathbf{E}_{1}^{* N}\left[\kappa_{\ell}, j_{\ell}\right]$ we deduce that $\left[\gamma_{\ell}+\right.$ $\left.\delta_{\ell}, i_{\ell}\right] \notin \mathbf{E}_{1}^{* N}[\mathbf{0}, i]$ and the proof of Theorem 6.23 applies. This yields a complementary 
component $U_{0}$ of $\mathcal{T}(i)$ containing an inner point of $\mathbf{h}^{N}\left(\mathcal{T}\left(i_{\ell}\right)+\gamma_{\ell}+\delta_{\ell}\right)$ for some $\ell \in\{0,1,2\}$ (see the proof of Theorem 6.23). From $\left[\gamma_{\ell}+\delta_{\ell}, i_{\ell}\right] \in \mathbf{E}_{1}^{* N}\left[\kappa_{\ell}, j_{\ell}\right]$ and the $N$-th iteration of the GIFS equation $\mathcal{T}\left(j_{\ell}\right)+\kappa_{\ell}=\bigcup_{[\lambda, k] \in\left(\mathbf{E}_{1}^{*}\right)^{N}\left[\kappa_{\ell}, j_{\ell}\right]} \mathbf{h}^{N}(\mathcal{T}(k)+\lambda)$ it follows that $\mathbf{h}^{N}\left(\mathcal{T}\left(i_{\ell}\right)+\gamma_{\ell}+\delta_{\ell}\right) \subset \mathcal{T}\left(j_{\ell}\right)+\kappa_{\ell}$ so that $U_{0}$ intersects the interior of $\mathcal{J}\left(j_{\ell}\right)+\kappa_{\ell}$. Let us denote by $\mathbf{z}$ a point contained in this intersection, i.e.,

$$
\mathbf{z} \in U_{0} \cap \operatorname{int}\left(\mathcal{J}\left(j_{\ell}\right)+\kappa_{\ell}\right), \quad U_{0} \text { bounded complementary component of } \mathcal{T}(i) .
$$

Now, comparing the definition of the edges in the SR-boundary graph (Definition 5.4) with the dual substitution $\mathbf{E}_{1}^{*}$ (Definition 3.4), we get the following correspondence. If there is an edge of type 1 from $[i, \gamma, j]$ to $\left[i^{\prime}, \gamma^{\prime}, j^{\prime}\right]$ labelled by $\eta$, we have $\eta=\pi \mathbf{l}\left(p_{1}\right)$ with $\sigma\left(i^{\prime}\right)=p_{1} i s_{1}$. From Definition 3.4 we deduce that $\left[\mathbf{h}^{-1} \eta, i^{\prime}\right] \in \mathbf{E}_{1}^{*}[\mathbf{0}, i]$. We also have $\mathbf{h} \gamma^{\prime}=\gamma+\pi\left(\mathbf{l}\left(p_{2}\right)-\mathbf{l}\left(p_{1}\right)\right)$ with $\sigma\left(j^{\prime}\right)=p_{2} j s_{2}$. This implies that $\left[\mathbf{h}^{-1}\left(\gamma+\pi \mathbf{l}\left(p_{2}\right)\right), j^{\prime}\right] \in \mathbf{E}_{1}^{*}[\gamma, j]$ and $\mathbf{h}^{-1}\left(\gamma+\pi \mathbf{l}\left(p_{2}\right)\right)=\gamma^{\prime}+\mathbf{h}^{-1} \pi \mathbf{l}\left(p_{1}\right)=\gamma^{\prime}+\mathbf{h}^{-1} \eta$. Hence,

$$
\left[\mathbf{h}^{-1} \eta, i^{\prime}\right] \in \mathbf{E}_{1}^{*}[\mathbf{0}, i], \quad\left[\gamma^{\prime}+\mathbf{h}^{-1} \eta, j^{\prime}\right] \in \mathbf{E}_{1}^{*}[\gamma, j] .
$$

If the edge is of type 2 , we have $\eta=\pi \mathbf{l}\left(p_{2}\right)+\gamma$ with $\sigma\left(i^{\prime}\right)=p_{2} j s_{2}, \sigma\left(j^{\prime}\right)=p_{1} i s_{1}$ and $-\mathbf{h} \gamma^{\prime}=\gamma+\pi \mathbf{l}\left(p_{2}\right)-\pi \mathbf{l}\left(p_{1}\right)=\eta-\pi \mathbf{l}\left(p_{1}\right)$. From $\sigma\left(j^{\prime}\right)=p_{1} i s_{1}$ we have $\left[\mathbf{h}^{-1} \pi \mathbf{l}\left(p_{1}\right), j^{\prime}\right] \in$ $\mathbf{E}_{1}^{*}[\mathbf{0}, i]$. From $\sigma\left(i^{\prime}\right)=p_{2} j s_{2}$ we have $\left[\mathbf{h}^{-1}\left(\pi \mathbf{l}\left(p_{2}\right)+\gamma\right), i^{\prime}\right] \in \mathbf{E}_{1}[\gamma, j]$. Hence,

$$
\left[\gamma^{\prime}+\mathbf{h}^{-1} \eta, j^{\prime}\right] \in \mathbf{E}_{1}^{*}[\mathbf{0}, i], \quad\left[\mathbf{h}^{-1} \eta, j^{\prime}\right] \in \mathbf{E}_{1}^{*}[\gamma, j] .
$$

From (6.16), (6.17), and the definition of $\mathbf{E}_{1}^{*}$, we deduce that if a walk of length $m$ leads from $[i, \gamma, j]$ to $\left[i^{\prime}, \gamma^{\prime}, j^{\prime}\right]$ with labels $\eta^{(1)}, \ldots, \eta^{(m)}$ with an even number of edges of type 2 , we have

$$
\begin{gathered}
{\left[\mathbf{h}^{-m} \eta^{(1)}+\cdots+\mathbf{h}^{-1} \eta^{(m)}, i^{\prime}\right] \in\left(\mathbf{E}_{1}^{*}\right)^{m}[\mathbf{0}, i],} \\
{\left[\gamma^{\prime}+\mathbf{h}^{-m} \eta^{(1)}+\cdots+\mathbf{h}^{-1} \eta^{(m)}, j^{\prime}\right] \in\left(\mathbf{E}_{1}^{*}\right)^{m}[\gamma, j]}
\end{gathered}
$$

(if the number of edges of type 2 was odd, we simply had to reverse $[\mathbf{0}, i]$ and $[\gamma, j]$ in the equation).

Let us apply this result to the walks from $\left[i, \overline{\kappa_{\ell}}, \overline{j_{\ell}}\right]$ to $\left[i, \kappa_{\ell}, j_{\ell}\right]$ that are supposed to exist by the hypotheses. We classify each walk by its length $L_{\ell}^{(m)}$ and we denote by $\zeta_{\ell}^{(m)}$ the sum $\mathbf{h}^{-L_{\ell}^{(m)}} \mathbf{e}^{(1)}+\cdots+\mathbf{h}^{-1} \eta^{\left(L_{\ell}^{(m)}\right)}$. This implies that

$$
\left[\zeta_{\ell}^{(m)}, i\right] \in\left(\mathbf{E}_{1}^{*}\right)^{L_{\ell}^{(m)}}[\mathbf{0}, i] \quad \text { and } \quad\left[\zeta_{\ell}^{(m)}+\kappa_{\ell}, j_{\ell}\right] \in\left(\mathbf{E}_{1}^{*}\right)^{L_{\ell}^{(m)}}\left[\overline{\kappa_{\ell}}, \overline{j_{\ell}}\right] .
$$

Since $\left[i, \overline{\kappa_{\ell}}, \overline{j_{\ell}}\right]$ is a node of the SR-boundary graph, the pair $\left[\overline{\kappa_{\ell}}, \overline{j_{\ell}}\right]$ belongs to $\Gamma_{\text {srs }}$ therefore $\left(\mathbf{E}_{1}^{*}\right)^{L_{\ell}^{(m)}}\left[\overline{\kappa_{\ell}}, \overline{j_{\ell}}\right]$ is disjoint from $\left(\mathbf{E}_{1}^{*}\right)^{L_{\ell}^{(m)}}[\mathbf{0}, i]$. Hence,

$$
\left[\zeta_{\ell}^{(m)}, i\right] \in\left(\mathbf{E}_{1}^{*}\right)^{L_{\ell}^{(m)}}[\mathbf{0}, i] \quad \text { and } \quad\left[\zeta_{\ell}^{(m)}+\kappa_{\ell}, j_{\ell}\right] \notin\left(\mathbf{E}_{1}^{*}\right)^{L_{\ell}^{(m)}}[\mathbf{0}, i] .
$$

Equation (3.4) and the tiling property we deduce that

$$
\mathbf{h}^{L_{\ell}^{(m)}}\left(\mathcal{T}(i)+\zeta_{\ell}^{(m)}\right) \subset \mathcal{T}(i) \quad \text { and } \quad \operatorname{int}\left(\mathbf{h}^{L_{\ell}^{(m)}}\left(\mathcal{T}\left(j_{\ell}\right)+\zeta_{\ell}^{(m)}+\kappa_{\ell}\right)\right) \cap \mathcal{T}(i)=\varnothing .
$$


From (6.15) we get

$\mathbf{h}^{L_{\ell}^{(m)}}\left(\mathbf{z}+\zeta_{\ell}^{(m)}\right) \in \mathbf{h}^{L_{\ell}^{(m)}}\left(U_{0}+\zeta_{\ell}^{(m)}\right)$ and $\mathbf{h}^{L_{\ell}^{(m)}}\left(\mathbf{z}+\zeta_{\ell}^{(m)}\right) \in \operatorname{int}\left(\mathbf{h}^{L_{\ell}^{(m)}}\left(\mathcal{T}\left(j_{\ell}\right)+\zeta_{\ell}^{(m)}+\kappa_{\ell}\right)\right)$, implying $\mathbf{h}^{L_{\ell}^{(m)}}\left(\mathbf{z}+\zeta_{\ell}^{(m)}\right) \notin \mathcal{T}(i)$. This yields that $\mathbf{h}^{L_{\ell}^{(m)}}\left(U_{0}+\zeta_{\ell}^{(m)}\right)$ intersects a complementary component $U_{m}$ of $\mathcal{T}(i)$.

Since $\mathbf{h}^{L_{\ell}^{(m)}}\left(\mathcal{T}(i)+\zeta_{\ell}^{(m)}\right) \subset \mathcal{T}(i)$, each complementary component of $\mathcal{T}(i)$ is a subset of exactly one complementary component of $\mathbf{h}^{L_{\ell}^{(m)}}\left(\mathcal{T}(i)+\zeta_{\ell}^{(m)}\right)$. As $\mathbf{h}^{L_{\ell}^{(m)}}\left(U_{0}+\right.$ $\left.\zeta_{\ell}^{(m)}\right)$ is a complementary component of $\mathbf{h}^{L_{\ell}^{(m)}}\left(\mathcal{J}(i)+\zeta_{\ell}^{m}\right)$, we deduce that

$$
U_{m} \subset \mathbf{h}^{L_{\ell}^{(m)}}\left(U_{0}+\zeta_{\ell}^{(m)}\right) .
$$

We thus have exhibited a sequence $\left(U_{m}\right)$ of complementary components of $\mathcal{T}(i)$ whose diameters tend to zero. Thus, there is an infinite subsequence of $\left(U_{m}\right)$ consisting of pairwise disjoint complementary components of $\mathcal{J}(i)$. So $\mathbb{S}^{2} \backslash \mathcal{J}(i)$ has infinitely many components. The result now follows from Lemma 6.22.

Items (1), (2.i) and (2.ii) of Theorem 6.26 can be checked in the same way as for Theorem 6.23. Item (2.iii) can be checked by inspecting the loops of the SR-boundary graph.

REMARK 6.27. - Note that we get analogs of Theorems 6.23 and 6.26 for $\mathcal{J}$ instead of $\mathcal{J}(i)$ by replacing $\mathbf{E}_{1}^{* N}[\mathbf{0}, i]$ by $\mathbf{E}_{1}^{* N} \mathcal{U}$. The proof remains exactly the same.

EXAMPLE 6.28. - In this example we deal with the substitution $\sigma_{6}(1)=12, \sigma_{6}(2)=$ $31, \sigma_{6}(3)=1$. We want to apply Theorem 6.26 to prove that the fundamental group of $\mathcal{T}(1)$ is uncountable.

To this matter take $i=1$ and consider the elements $[\mathbf{0}, 2],[\pi(1,-1,0), 3]$ and $[\pi(1,0,-1), 1]$ of $\Gamma_{\text {srs }}$. We will now check the items of Theorem 6.26 .

Item (1) : First observe that Proposition 5.33 implies that

$$
\mathcal{J}(2) \cap(\mathcal{T}(3)+\pi(1,-1,0)) \cap(\mathcal{T}(1)+\pi(1,0,-1))
$$

has infinitely many elements. Indeed, the node $[2, \pi(1,-1,0), 3, \pi(1,0,-1), 1]$ of the triple point graph is the starting point of infinitely many infinite walks (see Figure 6.6). At least one of these elements is contained in the interior of

$$
\mathcal{J}(2) \cap(\mathcal{T}(3)+\pi(1,-1,0)) \cap(\mathcal{T}(1)+\pi(1,0,-1))
$$

because the quadruple point graph accepts only a finite number of walks (see Figure 6.7).

Item 2.(i) : Take

$$
\left[\kappa_{0}, j_{0}\right]=\left[\kappa_{1}, j_{1}\right]=\left[\kappa_{2}, j_{2}\right]=[\pi(1,0,-1), 1] .
$$

We check that $[2, \pi(1,0,-1), 1]$ appears in the SR-boundary graph (see Figure 6.5). 
Item 2.(ii) : Take the translation vectors $\delta_{0}=\pi(6,-9,3), \delta_{1}=\pi(4,-7,4), \delta_{2}=$ $\pi(4,-8,5)$. This item holds for these vectors with $N=9$. Indeed, let $\gamma_{0}=\mathbf{0}$, $\gamma_{1}=\pi(1,-1,0)$ and $\gamma_{2}=\pi(1,0,-1)$. One checks that

(a) $[\pi(6,-9,3), 2]=\left[\delta_{0}+\gamma_{0}, 2\right] \in\left(\mathbf{E}_{1}^{*}\right)^{9}[\pi(1,0,-1), 1],[\pi(7,-10,3), 3]=\left[\delta_{0}+\right.$ $\left.\gamma_{1}, 3\right] \in\left(\mathbf{E}_{1}^{*}\right)^{9}[\mathbf{0}, 2]$ and $[\pi(7,-9,2), 1]=\left[\delta_{0}+\gamma_{2}, 1\right] \in\left(\mathbf{E}_{1}^{*}\right)^{9}[\mathbf{0}, 2]$.

(b) $[\pi(5,-8,4), 3]=\left[\delta_{1}+\gamma_{1}, 3\right] \in\left(\mathbf{E}_{1}^{*}\right)^{9}[\pi(1,0,-1), 1],[\pi(4,-7,4), 2]=\left[\delta_{1}+\right.$ $\left.\gamma_{0}, 2\right] \in\left(\mathbf{E}_{1}^{*}\right)^{9}[\mathbf{0}, 2]$ and $[\pi(5,-7,3), 1]=\left[\delta_{1}+\gamma_{2}, 1\right] \in\left(\mathbf{E}_{1}^{*}\right)^{9}[\mathbf{0}, 2]$.

(c) $[\pi(5,-8,4), 1]=\left[\delta_{2}+\gamma_{2}, 1\right] \in\left(\mathbf{E}_{1}^{*}\right)^{9}[\pi(1,0,-1), 1],[\pi(4,-8,5), 2]=\left[\delta_{2}+\right.$ $\left.\gamma_{0}, 2\right] \in\left(\mathbf{E}_{1}^{*}\right)^{9}[\mathbf{0}, 2]$ and $[\pi(5,-9,5), 3]=\left[\delta_{2}+\gamma_{1}, 3\right] \in\left(\mathbf{E}_{1}^{*}\right)^{9}[\mathbf{0}, 2]$.

A geometrical illustration is given in Figure 6.4.

Item 2.(iii) : The node $[2, \pi(1,0,-1), 1]$ appears in the SR-boundary graph and belongs to a loop, implying that an infinite number of walks with an even number of edges of type 2 start and end at this node (see Figure 6.5). Taking

$$
\left[i, \overline{\kappa_{0}}, \overline{j_{0}}\right]=\left[i, \overline{\kappa_{1}}, \overline{j_{1}}\right]=\left[i, \overline{\kappa_{2}}, \overline{j_{2}}\right]=[2, \pi(1,0,-1), 1]
$$

this item is shown to hold.

Summing up, the conditions of Theorem 6.26 are satisfied and we deduce that the fundamental group of $\mathcal{T}(1)$ is uncountable and not free.

ExAmPLE 6.29. - Theorem 6.26 also applies to $\sigma_{5}(1)=123, \sigma_{5}(2)=1, \sigma_{5}(3)=31$. For instance, we can show that $\mathcal{J}(1)$ has uncountable fundamental group in the following way.

First take $i=1,\left[\gamma_{1}, i_{1}\right]=[\pi(-1,1,1), 3]$ and $\left[\gamma_{2}, i_{2}\right]=[\pi(0,1,0), 1]$. Set $\left[\overline{\kappa_{\ell}}, \overline{j_{\ell}}\right]=$ $\left[\kappa_{\ell}, j_{\ell}\right]=[\mathbf{0}, 3](0 \leq \ell \leq 2)$. Moreover, choose $\delta_{0}=[\pi(1,2,-2), 1], \delta_{1}=[\pi(1,1,-1), 3]$ and $\delta_{2}=[\pi(1,1,-1), 1]$ for the translation vectors and take $N=7$, i.e., look at 7 fold iterations of $\mathbf{E}_{1}^{*}$. By considering the SR-boundary graph, the triple point graph and the quadruple point graph (see Figures 6.2,6.3, and 6.8) one easily checks that Theorem 6.26 applies. 

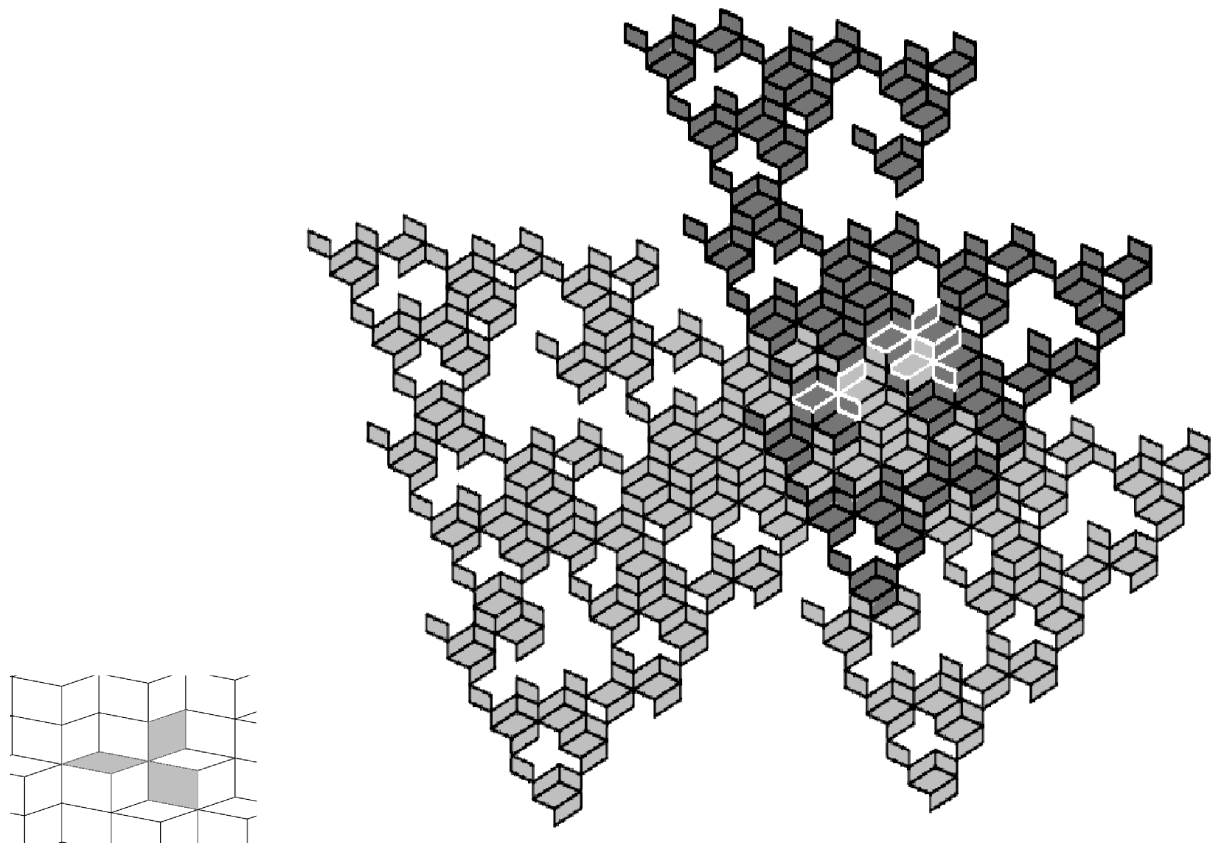

Figure 6.4. Consider the substitution $\sigma_{6}(1)=12, \sigma_{6}(2)=31, \sigma_{6}(3)=1$. The figure contains a geometric representation of $\left(\mathbf{E}_{1}^{*}\right)^{9}[\mathbf{0}, 2]$ (dark grey) and $\left(\mathbf{E}_{1}^{*}\right)^{9}[\pi(1,0,-1), 1]$ (light grey) among the self-replicating translation set $\Gamma_{\text {srs }}$. The geometrical representation as tips of the pieces $[\mathbf{0}, 2],[\pi(1,-1,0), 3],[\pi(1,0,1),-1]$ (depicted on the left hand side) can be translated such that two pieces of the shape belong to $\left(\mathbf{E}_{1}^{*}\right)^{9}[\mathbf{0}, 2]$ and the third belongs to $\left(\mathbf{E}_{1}^{*}\right)^{9}[\pi(1,0,-1), 1]$. The appropriate translation vectors are the ones given in Example 6.28. This proves Item (2.ii) of Theorem 6.26. 


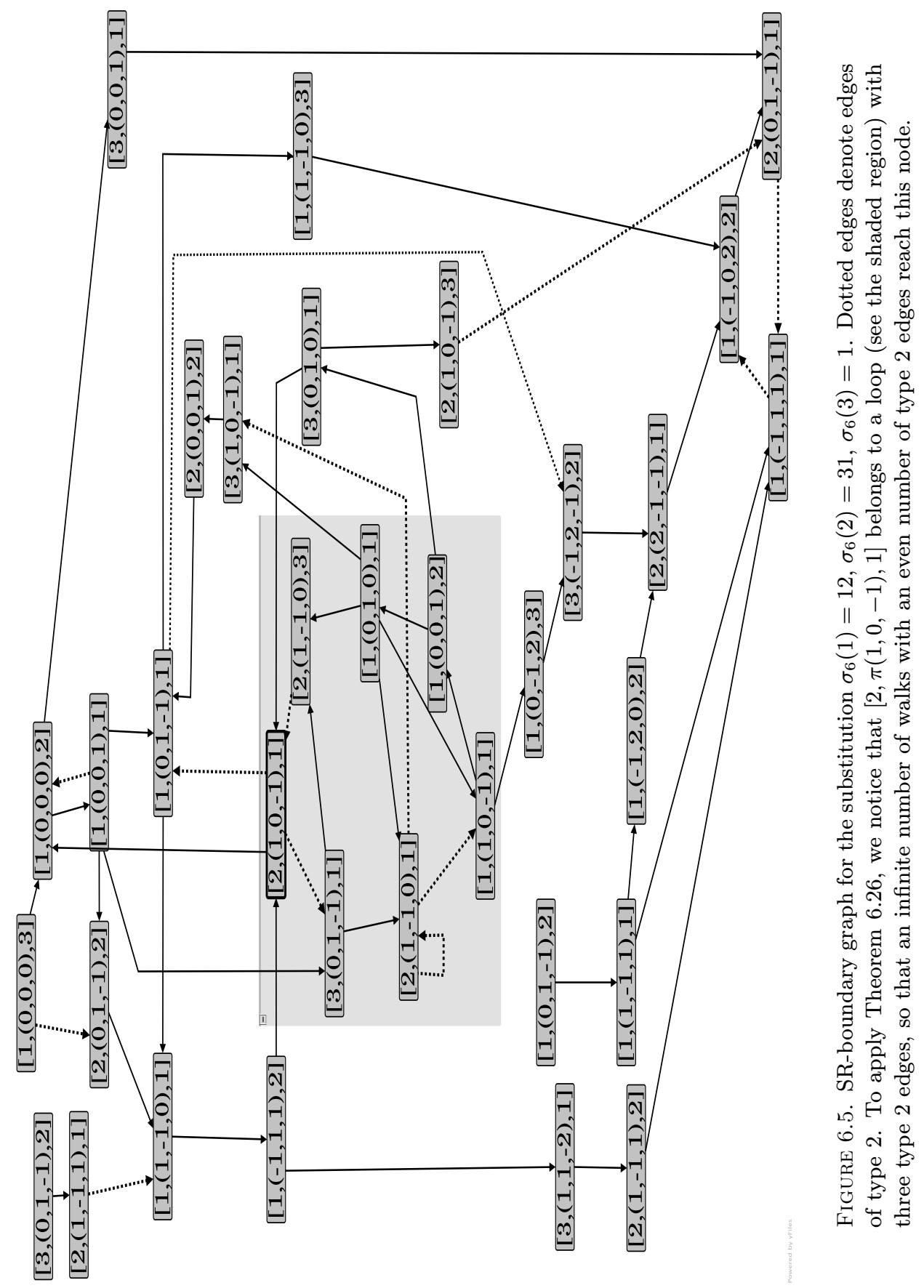




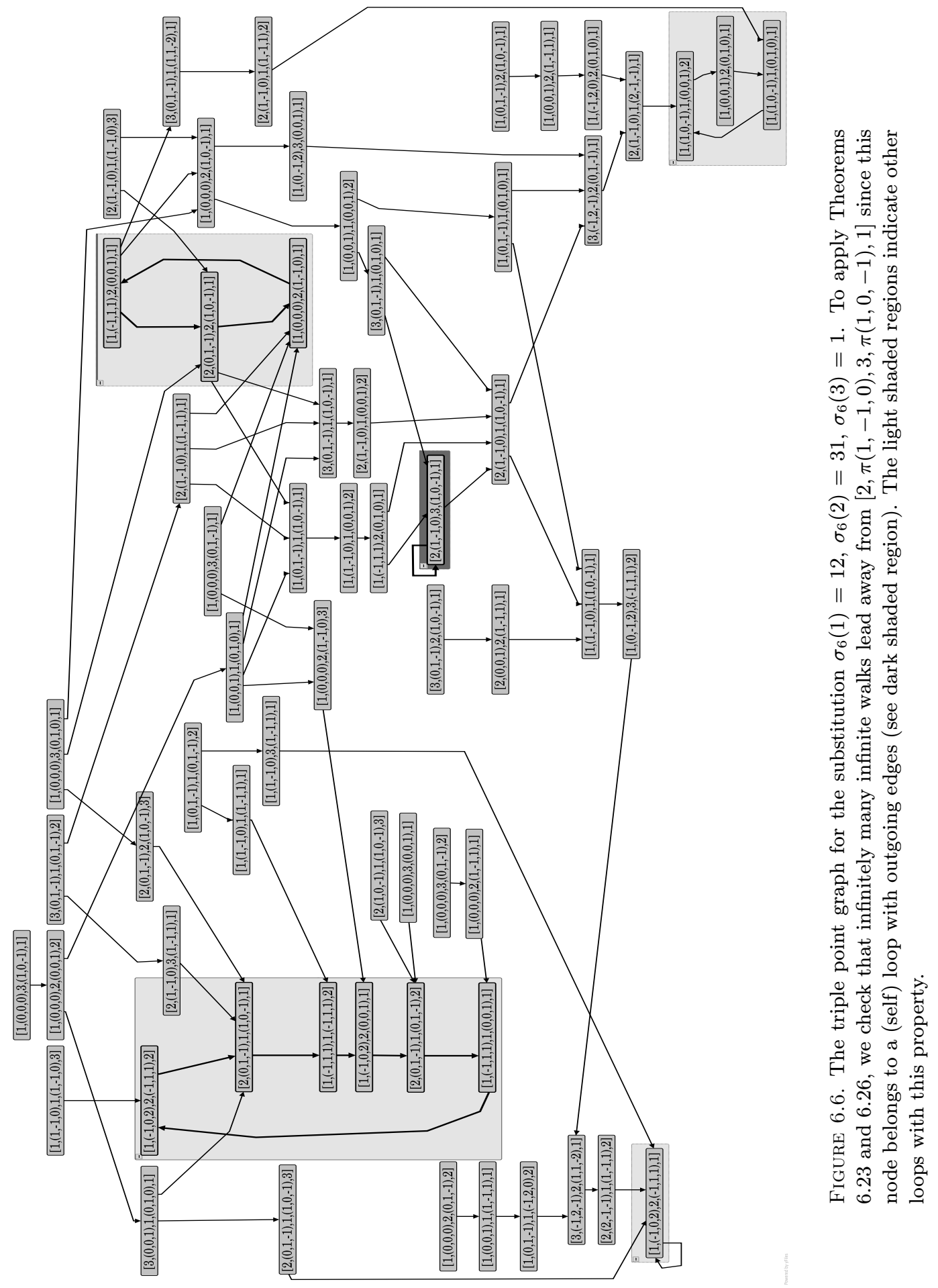




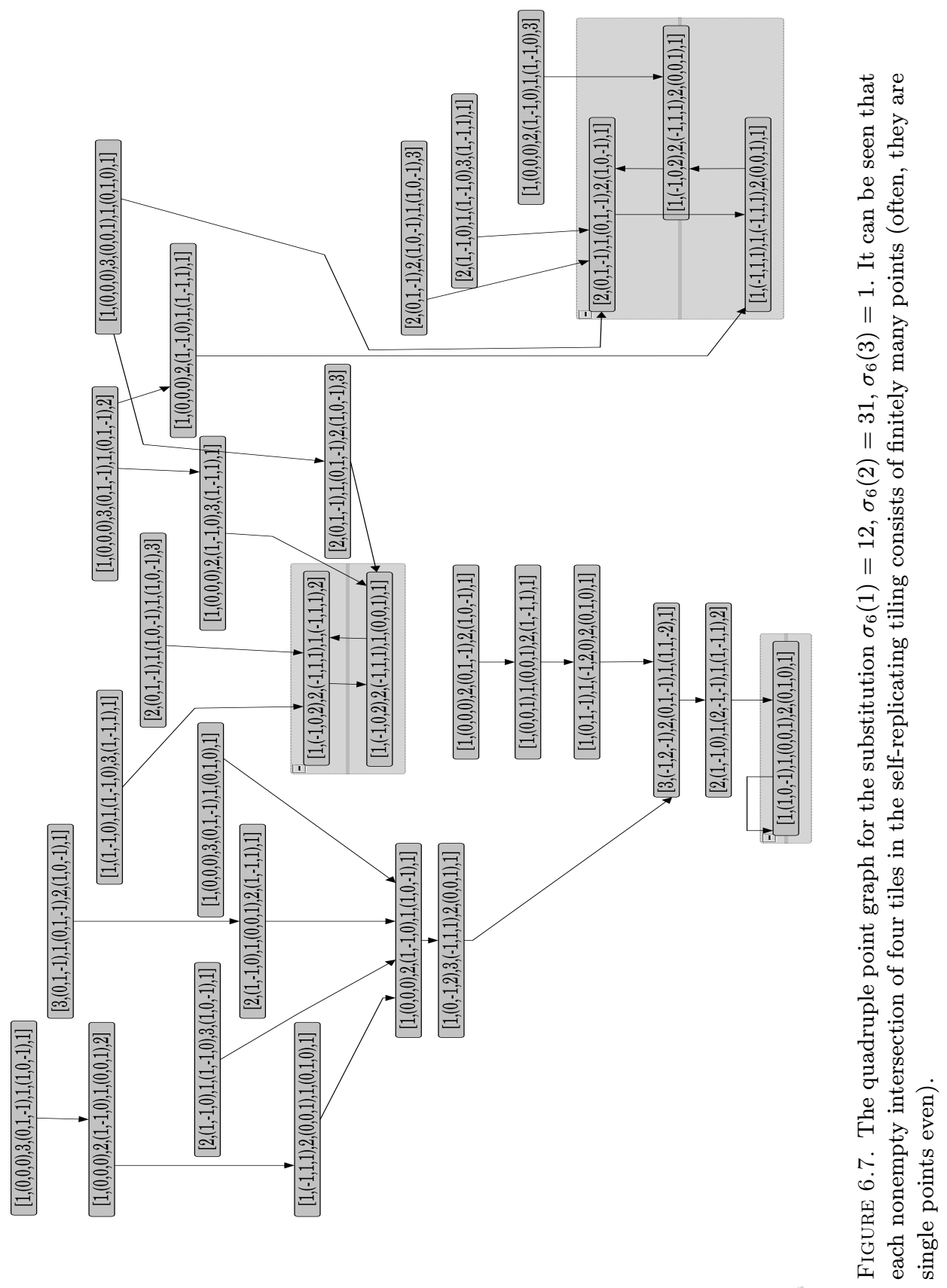




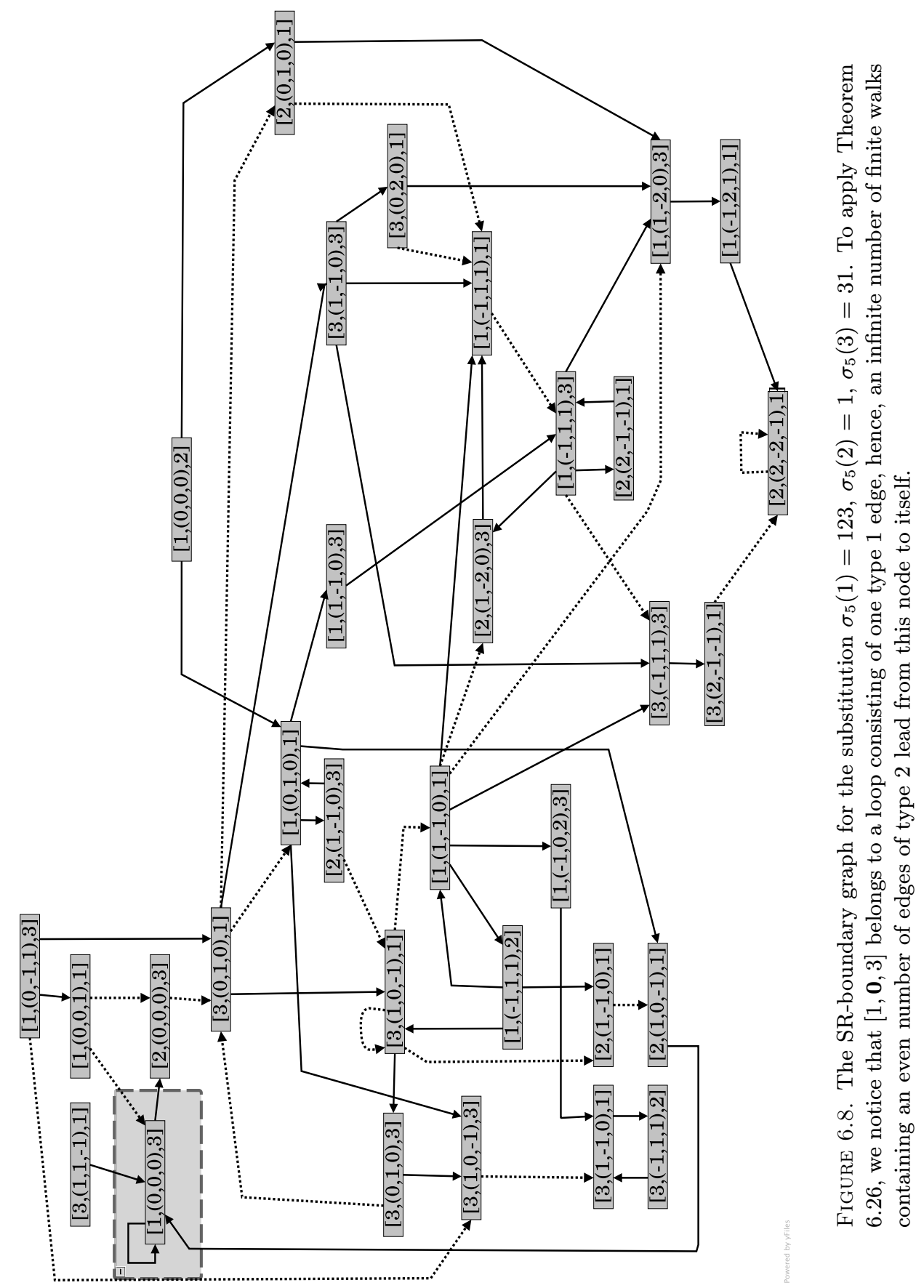




\section{CHAPTER 7}

\section{TECHNICAL PROOFS AND DEFINITIONS}

This chapter is devoted to detailed technical proofs of results we stated throughout this monograph.

\subsection{A technical proof from Chapter 3}

Proof of Proposition 3.15. - We introduce the lattice $\mathscr{L}=\sum_{k=2}^{d} \mathbb{Z}\left(\pi\left(\mathbf{e}_{B(k)}\right)-\right.$ $\left.\pi\left(\mathbf{e}_{B(1)}\right)\right)$ and the quotient mapping $\varphi: \mathbb{H}_{c} \rightarrow \mathbb{T}=\mathbb{H}_{c} / \mathcal{L}$. In order to apply the scheme of the proof of Proposition 3.11, we first need to check that the quotient mapping condition implies the following assertions.

1. $\mathcal{L}$ is indeed a lattice, that is, the generating family of $\mathscr{L}$ has rank $d-1$ in $\mathbb{H}_{c}$.

2. $\varphi\left(\left\{\pi\left(\mathbf{e}_{i}\right) ; 1 \leq i \leq n\right\}\right)$ is reduced to a single point denoted by $\mathbf{t}$.

3 . The addition of $\mathbf{t}$ is ergodic on the torus $\mathbb{T}$. The Kroneker theorem implies that this is true as soon as $\pi\left(\mathbf{e}_{B(1)}\right)$ is rationally independent from the generators of $\mathscr{L}$.

Let $\pi_{e+c}$ denote the projection of $\mathbb{R}^{n}$ on $\mathbb{H}_{c} \oplus \mathbb{H}_{e}$ along $\mathbb{H}_{s}$. We know that the left expanding eigenvector $\mathbf{v}_{\beta}$ of $\mathbf{M}$ is orthogonal to $\mathbb{H}_{c} \oplus \mathbb{H}_{s}$. Thus an explicit formula for $\pi_{e+c}$ is given by

$$
\forall \mathbf{x} \in \mathbb{R}^{n}, \quad \pi_{e+c}(\mathbf{x})=\pi(\mathbf{x})+\left\langle\mathbf{x}, \mathbf{v}_{\beta}\right\rangle \mathbf{u}_{\beta}=\sum_{i=1}^{d}\left\langle\mathbf{x}, \mathbf{v}_{\beta^{(i)}}\right\rangle \mathbf{u}_{\beta^{(i)}} \quad\left(\text { here } \beta=\beta^{(1)}\right) .
$$

From the quotient mapping condition (see (3.12)) we know that

$$
\left\langle\mathbf{e}_{j}, \mathbf{v}_{\beta}\right\rangle \in \sum_{k=1}^{d} \mathbb{Z}\left\langle\mathbf{e}_{B(k)}, \mathbf{v}_{\beta}\right\rangle \quad(j \in\{1, \ldots, n\}) .
$$

By applying the Galois morphisms this implies that also

$$
\left\langle\mathbf{e}_{j}, \mathbf{v}_{\beta^{(i)}}\right\rangle \in \sum_{k=1}^{d} \mathbb{Z}\left\langle\mathbf{e}_{B(k)}, \mathbf{v}_{\beta^{(i)}}\right\rangle \quad(j \in\{1, \ldots, n\})
$$


holds for each conjugate $\beta^{(i)}$ of $\beta$. We now apply (7.1) and deduce from this that $\pi_{e+c}\left(\mathbf{e}_{j}\right) \in \sum_{k=1}^{d} \mathbb{Z} \pi_{e+c}\left(\mathbf{e}_{B(k)}\right)$. Hence, the family $\left\{\pi_{e+c}\left(\mathbf{e}_{B(1)}\right), \ldots, \pi_{e+c}\left(\mathbf{e}_{B(d)}\right)\right\}$ is a generating family of the $d$-dimensional space $\mathbb{H}_{c} \oplus \mathbb{H}_{e}$. Thus it is a basis of this space.

Let $j \in\{1, \ldots, n\}$ be arbitrary. The representation (7.1) of $\pi_{e+c}$ implies that the coordinates of $\pi_{e+c}\left(\mathbf{e}_{j}\right)$ are invariant under the application of any Galois morphism of $\beta$. Thus these points have rational coordinates. We denote by $\left\{\mathbf{z}_{1}, \ldots, \mathbf{z}_{d}\right\}$ the dual basis in $\mathbb{H}_{c} \oplus \mathbb{H}_{e}$ of $\left\{\pi_{e+c}\left(\mathbf{e}_{B(1)}\right), \ldots, \pi_{e+c}\left(\mathbf{e}_{B(d)}\right)\right\}$. Linear algebra considerations imply that the elements $\mathbf{z}_{1}, \ldots, \mathbf{z}_{d}$ exist and also have rational coordinates.

To prove Item (1), assume that a linear combination of $\pi\left(\mathbf{e}_{B(2)}\right)-\pi\left(\mathbf{e}_{B(1)}\right), \ldots$, $\pi\left(\mathbf{e}_{B(d)}\right)-\pi\left(\mathbf{e}_{B(1)}\right)$ equals zero. Then we have $\sum_{k=1}^{d} \lambda_{k} \pi\left(\mathbf{e}_{B(k)}\right)=\mathbf{0}$ with $\sum_{k=1}^{d} \lambda_{k}=$ 0 . Lifting this to the space $\mathbb{H}_{c} \oplus \mathbb{H}_{e}$, we deduce that $\sum_{k=1}^{d} \lambda_{k} \pi_{e+c}\left(\mathbf{e}_{B(k)}\right)=\nu \mathbf{u}_{\beta}$. Let us compute the scalar product of $\mathbf{u}_{\beta}$ with the sum of the dual basis vectors $\mathbf{z}=\mathbf{z}_{1}+\cdots+\mathbf{z}_{d}$. This yields $\nu\left\langle\mathbf{u}_{\beta}, \mathbf{z}\right\rangle=\sum_{k=1}^{d} \lambda_{k}=0$. If $\left\langle\mathbf{u}_{\beta}, \mathbf{z}\right\rangle=0$, we apply Galois morphisms to this relation. Since $\mathbf{z}$ is rational, we obtain that each $\mathbf{u}_{\beta^{(i)}}$ is orthogonal to $\mathbf{z}$, hence $\mathbf{z}$ is orthogonal to the full space $\mathbb{H}_{c} \oplus \mathbb{H}_{e}$ which is impossible. Hence, $\nu=0$ and, since $\left\{\pi_{e+c}\left(\mathbf{e}_{B(1)}\right), \ldots, \pi_{e+c}\left(\mathbf{e}_{B(d)}\right)\right\}$ is a basis of $\mathbb{H}_{c} \oplus \mathbb{H}_{e}, \lambda_{k}=0$ for each $k \in\{1, \ldots, d\}$. This implies Item (1).

To prove Item (2) we have to show that $\varphi\left(\pi\left(\mathbf{e}_{j}\right)\right)=\varphi\left(\pi\left(\mathbf{e}_{B(1)}\right)\right)$ holds for each $j \in$ $\{1, \ldots, n\}$. To this matter observe that conjugating the quotient mapping condition implies that

$$
\left\langle\mathbf{e}_{j}-\mathbf{e}_{B(1)}, \mathbf{v}_{\beta^{(i)}}\right\rangle \in \sum_{k=2}^{d} \mathbb{Z}\left\langle\mathbf{e}_{B(k)}-\mathbf{e}_{B(1)}, \mathbf{v}_{\beta^{(i)}}\right\rangle .
$$

By the representation of $\pi$ in (2.4) this implies that $\pi\left(\mathbf{e}_{j}-\mathbf{e}_{B(1)}\right) \in \mathcal{L}$. Since $\varphi$ takes its arguments modulo $\mathscr{L}$ this implies that $\varphi\left(\pi\left(\mathbf{e}_{j}\right)\right)=\varphi\left(\pi\left(\mathbf{e}_{B(1)}\right)\right)$. As $j$ was arbitrary this yields Item (2).

Proving Item (3) is equivalent to proving rational independency between $\pi\left(\mathbf{e}_{B(1)}\right)$ and $\pi\left(\mathbf{e}_{B(2)}\right)-\pi\left(\mathbf{e}_{B(1)}\right), \ldots, \pi\left(\mathbf{e}_{B(d)}\right)-\pi\left(\mathbf{e}_{B(1)}\right)$. Assume that $\sum_{k=2}^{d} \lambda_{k} \pi\left(\mathbf{e}_{B(k)}\right)=$ $\left(\lambda_{d}+\sum_{k=1}^{d-1} \lambda_{k}\right) \pi\left(\mathbf{e}_{B(1)}\right)$ with $\lambda_{k} \in \mathbb{Q}$. By using (2.5), we extend this relation to a rational dependency between $\pi_{e+c}\left(\mathbf{e}_{B(1)}\right), \ldots, \pi_{e+c}\left(\mathbf{e}_{B(d)}\right)$. Since these vectors are linearly independent, we deduce that $\lambda_{k}=0$ for every $k$ and Item (3) is proved.

Set $\mathbf{t}=\varphi\left(\pi\left(\mathbf{e}_{B(1)}\right)\right)$ and let $\psi(\mathbf{x})$ be the number of tiles that contain a given point $\mathbf{x}$. Note that by the definition of the subtiles $\mathcal{T}(i)$ the function $\psi$ is a measurable function which is invariant under the rotation by $\mathbf{t}$. By the ergodicity of the rotation by $\mathbf{t}$ the mapping $\psi$ is constant almost everywhere. This implies that the covering $\bigcup_{[\gamma, i] \in \Gamma_{\text {lat }}}(\mathcal{T}(i)+\gamma)$ of $\mathbb{H}_{c}$ is a multiple tiling (for details see the proof of Host in the irreducible case which is sketched in [86, Exercise 7.5.14]). 


\subsection{Technical proofs from Chapter 5}

Proof of Proposition 5.2. - The nodes of $\mathscr{G}^{(\mathbf{0})}$ are elements of $\Gamma_{\text {srs }}$. Since this set is a Delaunay set, the graph has to be finite by Condition (1) of its definition.

Consider a node $[\gamma, i]$ of $\mathscr{G}^{(\mathbf{0})}$. There exists an infinite walk $\left(\left[\gamma_{k}, i_{k}\right]\right)_{k \geq 0}$ starting from $\left[\gamma_{0}, i_{0}\right]=[\gamma, i]$. Let $\left(p_{k}, i_{k}, s_{k}\right)$ with $\sigma\left(i_{k+1}\right)=p_{k} i_{k} s_{k}(k \geq 0)$ be the labelling of this walk. By considering the definition of the edges of $\mathscr{G}^{(\mathbf{0})}$, we obtain an expansion of $\gamma$ as

$$
\gamma=-\pi \mathbf{l}\left(p_{0}\right)-\mathbf{h} \pi \mathbf{l}\left(p_{1}\right)-\cdots-\mathbf{h}^{k} \pi \mathbf{l}\left(p_{k}\right)+\mathbf{h}^{k+1} \gamma_{k+1} \quad(k \geq 0) .
$$

Since $\left\|\gamma_{k}\right\|$ is uniformly bounded in $k$ and $\mathbf{h}$ is a contraction, for $k \rightarrow \infty$ we obtain a convergent power series. Thus $\gamma=-\sum_{k \geq 0} \mathbf{h}^{k} \pi \mathbf{l}\left(p_{k}\right)$. This means that $-\gamma$ can be expanded by an h-ary representation of a walk of the prefix-suffix graph starting in the node $i$. By Corollary 2.8 this implies that $-\gamma \in \mathcal{J}(i)$ and, hence, $\mathbf{0} \in \mathcal{J}(i)+\gamma$.

Suppose conversely that $\mathbf{0} \in \mathcal{J}(i)+\gamma$ with $[\gamma, i] \in \Gamma_{\text {srs }}$. Then by Corollary 2.8 $-\gamma$ can be expanded as $-\gamma=\sum_{k \geq 0} \mathbf{h}^{k} \pi \mathbf{l}\left(p_{k}\right)$, with $\left(p_{k}, i_{k}, s_{k}\right)_{k \geq 0}$ a suitable walk in the prefix-suffix graph starting in $i$. Let $\gamma_{\ell}=-\sum_{k \geq 0} \mathbf{h}^{k} \pi \mathbf{l}\left(p_{k+\ell}\right)$. Each $\gamma_{\ell}$ obviously satisfies (5.1) and $\mathbf{h} \gamma_{\ell+1}=\gamma_{\ell}+\pi \mathbf{l}\left(p_{\ell}\right)$.

We now deduce by induction that $\left[\gamma_{\ell}, i_{\ell}\right] \in \Gamma_{\text {srs }}$ for all $\ell \in \mathbb{N}$. As $\gamma=\gamma_{0}$, the induction start is clear, to perform the induction step assume that $\left[\gamma_{\ell}, i_{\ell}\right] \in \Gamma_{\mathrm{srs}}$ with $\mathbf{x}_{\ell}$ chosen in a way that $\gamma=\pi\left(\mathbf{x}_{\ell}\right)$ and consider $\left[\gamma_{\ell+1}, i_{\ell+1}\right]$. Indeed, $\gamma_{\ell+1}=\pi\left(\mathbf{x}_{\ell+1}\right)$ with $\mathbf{x}_{\ell+1}=\mathbf{M}^{-1}\left(\mathbf{l}\left(p_{\ell}\right)+\mathbf{x}_{\ell}\right)$ and, hence, $\gamma_{\ell+1} \in \pi\left(\mathbb{Z}^{n}\right)$ by the unimodularity of $\mathbf{M}$. It remains to show that $0 \leq\left\langle\mathbf{x}_{\ell+1}, \mathbf{v}_{\beta}\right\rangle\left\langle\left\langle\mathbf{e}_{i_{\ell+1}}, \mathbf{v}_{\beta}\right\rangle\right.$ (assuming by induction that this relation is satisfied for $\left[\pi\left(\mathbf{x}_{\ell}\right), i_{\ell}\right]$ ). Indeed, we have (note that $\mathbf{e}_{j}=\mathbf{l}(j)$ for each $j \in \mathscr{C})$

$$
\begin{aligned}
\left\langle\mathbf{x}_{\ell+1}, \mathbf{v}_{\beta}\right\rangle & =\left\langle\mathbf{M}^{-1}\left(\mathbf{l}\left(p_{\ell}\right)+\mathbf{x}_{\ell}\right), \mathbf{v}_{\beta}\right\rangle=\beta^{-1}\left\langle\mathbf{l}\left(p_{\ell}\right)+\mathbf{x}_{\ell}, \mathbf{v}_{\beta}\right\rangle\left\langle\beta^{-1}\left\langle\mathbf{l}\left(p_{\ell}\right)+\mathbf{e}_{i_{\ell}}, \mathbf{v}_{\beta}\right\rangle\right. \\
& =\beta^{-1}\left\langle\mathbf{l}\left(p_{\ell}\right)+\mathbf{l}\left(i_{\ell}\right), \mathbf{v}_{\beta}\right\rangle \leq \beta^{-1}\left\langle\mathbf{l}\left(\sigma\left(i_{\ell+1}\right)\right), \mathbf{v}_{\beta}\right\rangle \\
& =\beta^{-1}\left\langle\mathbf{M l}\left(i_{\ell+1}\right), \mathbf{v}_{\beta}\right\rangle=\left\langle\mathbf{l}\left(i_{\ell+1}\right), \mathbf{v}_{\beta}\right\rangle=\left\langle\mathbf{e}_{i_{\ell+1}}, \mathbf{v}_{\beta}\right\rangle
\end{aligned}
$$

and

$$
\left\langle\mathbf{x}_{\ell+1}, \mathbf{v}_{\beta}\right\rangle=\left\langle\mathbf{M}^{-1}\left(\mathbf{l}\left(p_{\ell}\right)+\mathbf{x}_{\ell}\right), \mathbf{v}_{\beta}\right\rangle=\beta^{-1}\left\langle\mathbf{l}\left(p_{\ell}\right)+\mathbf{x}_{\ell}, \mathbf{v}_{\beta}\right\rangle \geq \beta^{-1}\left\langle\mathbf{l}\left(p_{\ell}\right), \mathbf{v}_{\beta}\right\rangle \geq 0 .
$$

Hence, $\left(\left[\gamma_{k}, i_{k}\right]\right)_{k \geq 0}$ is an infinite walk in the zero-expansion graph starting from $[\gamma, i]$.

Proof of Proposition 5.5. - Let $\&$ be finite and fixed. We first prove that every set of points $[i, \gamma, j]$ that satisfies conditions (1), (2) and (3) of Definition 5.4 for $\phi$ is finite. If $[i, \gamma, j]$ satisfies these conditions, there exists a finite walk $\left[i_{0}, \gamma_{0}, j_{0}\right] \rightarrow\left[i_{1}, \gamma_{1}, j_{1}\right] \rightarrow$ $\cdots\left[i_{k}, \gamma_{k}, j_{k}\right] \rightarrow[i, \gamma, j]$, where $\left[i_{0}, \gamma_{0}, j_{0}\right]$ is an element of the finite set $\phi$. For each $\ell \in\{0, \ldots, k\}$, choose $\mathbf{x}_{\ell} \in \mathbb{Z}^{n}$ in a way that $\gamma_{\ell}=\pi\left(\mathbf{x}_{\ell}\right)$. Moreover, choose $\mathbf{x}$ such that $\gamma=\pi(\mathbf{x})$. (Note that these choices are unique only in the case of irreducible unit Pisot substitutions.) 
From the definition of the edges of the boundary graph, we deduce that $\mathbf{h} \gamma=$ $\pi(\mathbf{M x})= \pm \gamma_{k}+\pi \mathbf{l}\left(p_{2}^{(k)}\right)-\pi \mathbf{l}\left(p_{1}^{(k)}\right)=\pi\left( \pm \mathbf{x}_{k}+\mathbf{l}\left(p_{2}^{(k)}\right)-\mathbf{l}\left(p_{1}^{(k)}\right)\right)$. By iterating this procedure and setting $\mathbf{l}\left(p^{(\ell)}\right)=\mathbf{l}\left(p_{2}^{(\ell)}\right)-\mathbf{l}\left(p_{1}^{(\ell)}\right)$, we obtain a representation of $\gamma$ as

$$
\gamma=\pi(\mathbf{x})=\pi\left( \pm \mathbf{M}^{-1} \mathbf{l}\left(p^{(k)}\right) \pm \cdots \pm \mathbf{M}^{-k} \mathbf{l}\left(p^{(1)}\right) \pm \mathbf{M}^{-k-1} \mathbf{l}\left(p^{(0)}\right) \pm \mathbf{M}^{-k-1} \mathbf{x}_{0}\right)
$$

(note that the signs \pm are independent from each other).

From (2.5) we deduce that

$$
\begin{aligned}
\left\langle\mathbf{x}, \mathbf{v}_{\beta}\right\rangle= & \pm\left\langle\mathbf{M}^{-1} \mathbf{l}\left(p^{(k)}\right), \mathbf{v}_{\beta}\right\rangle \pm \cdots \pm\left\langle\mathbf{M}^{-k} \mathbf{l}\left(p^{(1)}\right), \mathbf{v}_{\beta}\right\rangle \\
& \pm\left\langle\mathbf{M}^{-k-1} \mathbf{l}\left(p^{(0)}\right), \mathbf{v}_{\beta}\right\rangle \pm\left\langle\mathbf{M}^{-k-1} \mathbf{x}_{0}, \mathbf{v}_{\beta}\right\rangle \\
= & \pm \beta^{-1}\left\langle\mathbf{l}\left(p^{(k)}\right), \mathbf{v}_{\beta}\right\rangle \pm \cdots \pm \beta^{-k}\left\langle\mathbf{l}\left(p^{(1)}\right), \mathbf{v}_{\beta}\right\rangle \\
& \pm \beta^{-k-1}\left\langle\mathbf{l}\left(p^{(0)}\right), \mathbf{v}_{\beta}\right\rangle \pm \beta^{-k-1}\left\langle\mathbf{x}_{0}, \mathbf{v}_{\beta}\right\rangle
\end{aligned}
$$

Since $\left[i_{0}, \gamma_{0}, j_{0}\right]=\left[i_{0}, \pi\left(\mathbf{x}_{0}\right), j_{0}\right] \in \&$ the element $\gamma_{0}$ and, hence, also the element $\left\langle\mathbf{x}_{0}, \mathbf{v}_{\beta}\right\rangle$, can attain only finitely many different values. Thus $\left\langle\mathbf{x}, \mathbf{v}_{\beta}\right\rangle$ is uniformly bounded. In view of Condition (5.4) and by the definition of the norm in (2.1), $\left\langle\mathbf{x}, \mathbf{v}_{\beta^{(\ell)}}\right\rangle$ is also uniformly bounded for each contracting eigenvalue $\beta^{(\ell)}$.

Thus $\left\langle\mathbf{x}, \mathbf{v}_{\beta}\right\rangle$ belongs to a bounded subset of $\mathbb{Z}[\beta]$ all whose Galois conjugates are bounded. This subset has to be finite which implies that there are only finitely many possibilities for $\left\langle\mathbf{x}, \mathbf{v}_{\beta}\right\rangle$. Since in view of $(2.5) \gamma=\pi(\mathbf{x})$ is uniquely determined by $\left\langle\mathbf{x}, \mathbf{v}_{\beta}\right\rangle$ there are only finitely many possibilities for $\gamma$ and we are done.

Now each set of nodes that satisfies conditions (1), (2) and (3) is finite with a uniform bound. So the union of sets that satisfy these conditions also satisfies them, hence, it is finite with the same bound. We conclude that the boundary graph which is the largest graph satisfying (1), (2) and (3) is well defined and finite.

Proof of Theorem 5.6. - Suppose that $[i, \gamma, j]$ is a node of the boundary graph. By definition, $[i, \gamma, j]$ is the starting point of an infinite walk in the graph $\mathscr{G}^{(B)}(\phi)$ Let $[i, \gamma, j]=\left[i_{0}, \gamma_{0}, j_{0}\right] \rightarrow \cdots \rightarrow\left[i_{k}, \gamma_{k}, j_{k}\right] \rightarrow \cdots$ be this walk. The edges of this walk are either of type 1 or of type 2 . If we replace some of the nodes $\left[i_{k}, \gamma_{k}, j_{k}\right]$ with $\left[j_{k},-\gamma_{k}, i_{k}\right]$, we can associate to this walk a sequence of edges which only consists of type 1 edges. We denote this sequence of edges by $[i, \gamma, j]=\left[m_{0}, \alpha_{0}, n_{0}\right] \rightarrow \cdots \rightarrow$ $\left[m_{k}, \alpha_{k}, n_{k}\right] \rightarrow \cdots$. Note that this change has the effect that some of the elements $\left[m_{k}, \alpha_{k}, n_{k}\right]$ do not satisfy $\left[\alpha_{k}, n_{k}\right] \in \Gamma_{\text {srs }}$ which means that this sequence of edges is no longer a walk in the boundary graph. However, for this proof this is no drawback. Indeed, from this new sequence of edges we can easily build two sequences in the prefix-suffix graph $\left(p_{k}, m_{k}, s_{k}\right)_{k \geq 0}$ and $\left(p_{k}^{\prime}, n_{k}, s_{k}^{\prime}\right)_{k \geq 0}$ such that

$$
\mathbf{h} \alpha_{k}=\alpha_{k-1}+\pi \mathbf{l}\left(p_{k-1}^{\prime}\right)-\pi \mathbf{l}\left(p_{k-1}\right) .
$$

This yields

$$
\mathbf{h}^{k} \alpha_{k}=\gamma_{0}+\sum_{\ell=0}^{k-1} \mathbf{h}^{\ell} \pi \mathbf{l}\left(p_{\ell}^{\prime}\right)-\sum_{\ell=0}^{k-1} \mathbf{h}^{\ell} \pi \mathbf{l}\left(p_{\ell}\right)
$$


Since $\alpha_{k}$ belongs to a finite set and $\mathbf{h}$ is a uniform contraction on $\mathbb{H}_{c}$, we deduce that

$$
\gamma=\gamma_{0}=-\sum_{\ell \geq 0} \mathbf{h}^{\ell} \pi \mathbf{l}\left(p_{\ell}^{\prime}\right)+\sum_{\ell \geq 0} \mathbf{h}^{\ell} \pi \mathbf{l}\left(p_{\ell}\right) .
$$

But $\sum_{\ell \geq 0} \mathbf{h}^{\ell} \pi \mathbf{l}\left(p_{\ell}^{\prime}\right)$ is build from a walk of the prefix-suffix graph that starts in $j=j_{0}$, hence, by Corollary 2.8, $\sum_{\ell \geq 0} \mathbf{h}^{\ell} \pi \mathbf{l}\left(p_{\ell}^{\prime}\right) \in \mathcal{T}(j)$. Similarly, $\sum_{\ell \geq 0} \mathbf{h}^{\ell} \pi \mathbf{l}\left(p_{\ell}\right) \in \mathcal{T}(i)$. Hence, equation (7.2) implies that $\mathcal{J}(i) \cap(\mathcal{T}(j)+\gamma)$ is nonempty.

Conversely, if the intersection $\mathcal{T}(i) \cap \mathcal{T}(j)+\gamma$ is nonempty, we build an explicit infinite walk of the boundary graph starting in $[i, \gamma, j]$ in view of Corollary 2.8. More precisely, there exist two walks $\left(p_{k}, i_{k}, s_{k}\right)_{k \geq 0}$ and $\left(p_{k}^{\prime}, i_{k}^{\prime}, s_{k}^{\prime}\right)_{k \geq 0}$ in the prefix-suffix graph starting in $i$ and $j$, respectively, such that $\gamma=\sum_{k \geq 0} \mathbf{h}^{k} \pi \mathbf{l}\left(p_{k}\right)-\sum_{k \geq 0} \mathbf{h}^{k} \pi \mathbf{l}\left(p_{k}^{\prime}\right)$. We will now iteratively build an infinite walk of the boundary graph starting in $[i, \gamma, j]$.

Let $\gamma_{1}=\sum_{k \geq 0} \mathbf{h}^{k} \pi \mathbf{l}\left(p_{k+1}\right)-\sum_{k \geq 0} \mathbf{h}^{k} \pi \mathbf{l}\left(p_{k+1}^{\prime}\right)$. The points $\gamma_{1}$ and $-\gamma_{1}$ obviously satisfy (5.4). It remains to choose the possibility that produces a triple belonging to the set $\mathfrak{D}$. We know that we can choose $\mathbf{x} \in \mathbb{Z}^{n}$ such that $\gamma=\pi(\mathbf{x})$. From the definition of $\gamma_{1}$ we deduce that $\gamma_{1}=\pi\left(\mathbf{x}_{1}\right)$ with $\mathbf{M x}_{1}=\mathbf{x}+\mathbf{l}\left(p_{0}\right)-\mathbf{l}\left(p_{0}^{\prime}\right)$. If $\left\langle\mathbf{x}_{1}, \mathbf{v}_{\beta}\right\rangle>0$ then $\left[i_{1}, \gamma_{1}, j_{1}\right]$ belongs to $\mathfrak{D}$ and there is an edge of type 1 from $[i, \gamma, j]$ to $\left[i_{1}, \gamma_{1}, j_{1}\right]$. If $\left\langle\mathbf{x}_{1}, \mathbf{v}_{\beta}\right\rangle<0$, then $\left[j_{1},-\gamma_{1}, i_{1}\right]$ belongs to $\mathfrak{D}$ and there is an edge of type 2 from $[i, \gamma, j]$ to $\left[i_{1},-\gamma_{1}, j_{1}\right]$. If $\left\langle\mathbf{x}_{1}, \mathbf{v}_{\beta}\right\rangle=0$ and $i_{1} \leq j_{1}$, there is an edge of type 1 from $[i, \gamma, j]$ to $\left[i_{1}, \gamma_{1}, j_{1}\right]$. If $\left\langle\mathbf{x}_{1}, \mathbf{v}_{\beta}\right\rangle=0$ and $i_{1}>j_{1}$, there is an edge of type 2 from $[i, \gamma, j]$ to $\left[j_{1},-\gamma_{1}, i_{1}\right]$.

The process can be iterated and we obtain an infinite walk in the boundary graph starting in $[i, \gamma, j]$. Hence, $[i, \gamma, j]$ is a node of the boundary graph.

Proof of Theorem 5.7. - To prove the first assertion we need to check that for every node $[i, \gamma, j]$ of the SR-boundary graph, $[\gamma, j]$ belongs to the self-replicating set $\Gamma_{\text {srs }}$. This is done by induction on the length of the walk leading to a node from a starting node contained in $\phi_{\text {srs }}$. As the induction start is trivial, assume that $[\gamma, j] \in \Gamma_{\mathrm{srs}}$ and that there exists an edge from $[i, \gamma, j]$ to $\left[i_{1}, \gamma_{1}, j_{1}\right]$. If the edge is of type 1 we have $\gamma_{1}=\mathbf{h}^{-1}\left(\gamma+\pi \mathbf{l}\left(p_{2}\right)-\pi \mathbf{l}\left(p_{1}\right)\right)$ with $\sigma\left(j_{1}\right)=p_{2} j s_{2}$. Since $\left[i_{1}, \gamma_{1}, j_{1}\right]$ is a node of the graph, it belongs to $\mathfrak{D}$, hence, there is $\mathbf{x}_{1} \in \mathbb{Z}^{n}$ with $\gamma_{1}=\pi\left(\mathbf{x}_{1}\right)$ and $\left\langle\mathbf{x}_{1}, \mathbf{v}_{\beta}\right\rangle \geq 0$. Since $[\gamma, j] \in \Gamma_{\text {srs }}$, we also have $\gamma=\pi(\mathbf{x})$ for some $\mathbf{x} \in \mathbb{Z}^{n}$ with $0 \leq\left\langle\mathbf{x}, \mathbf{v}_{\beta}\right\rangle\left\langle\left\langle\mathbf{e}_{j}, \mathbf{v}_{\beta}\right\rangle\right.$. We deduce that $\pi\left(\mathbf{x}_{1}\right)=\pi\left(\mathbf{M}^{-1}\left(\mathbf{x}+\mathbf{l}\left(p_{2}\right)-\mathbf{l}\left(p_{1}\right)\right)\right)$. Then (2.5) implies that

$$
\begin{aligned}
\left\langle\mathbf{x}_{1}, \mathbf{v}_{\beta}\right\rangle & =\left\langle\mathbf{M}^{-1}\left(\mathbf{x}+\mathbf{l}\left(p_{2}\right)-\mathbf{l}\left(p_{1}\right)\right), \mathbf{v}_{\beta}\right\rangle=\beta^{-1}\left\langle\mathbf{x}+\mathbf{l}\left(p_{2}\right)-\mathbf{l}\left(p_{1}\right), \mathbf{v}_{\beta}\right\rangle \\
& <\beta^{-1}\left\langle\mathbf{e}_{j}+\mathbf{l}\left(p_{2}\right), \mathbf{v}_{\beta}\right\rangle \leq \beta^{-1}\left\langle\mathbf{l}\left(\sigma\left(j_{1}\right)\right), \mathbf{v}_{\beta}\right\rangle=\left\langle\mathbf{e}_{j_{1}}, \mathbf{v}_{\beta}\right\rangle .
\end{aligned}
$$

Hence $\left[\gamma_{1}, j_{1}\right] \in \Gamma_{\text {srs }}$.

Assume now that the edge from $[i, \gamma, j]$ to $\left[i_{1}, \gamma_{1}, j_{1}\right]$ is of type 2 . Then $\gamma_{1}=\pi\left(\mathbf{x}_{1}\right)$ with $\pi\left(\mathbf{x}_{1}\right)=\pi\left(\mathbf{M}^{-1}\left(-\mathbf{x}+\mathbf{l}\left(p_{1}\right)-\mathbf{l}\left(p_{2}\right)\right)\right)$ and $\sigma\left(j_{1}\right)=p_{1} i s_{1}$. We already know that $\left\langle\mathbf{x}_{1}, \mathbf{v}_{\beta}\right\rangle \geq 0$, and $\left\langle\mathbf{x}, \mathbf{v}_{\beta}\right\rangle \geq 0$. Moreover,

$$
\left\langle\mathbf{x}_{1}, \mathbf{v}_{\beta}\right\rangle \leq \beta^{-1}\left\langle\mathbf{l}\left(p_{1}\right), \mathbf{v}_{\beta}\right\rangle<\beta^{-1}\left\langle\mathbf{l}\left(p_{1} i s_{1}\right), \mathbf{v}_{\beta}\right\rangle=\beta^{-1}\left\langle\mathbf{l}\left(\sigma\left(j_{1}\right)\right), \mathbf{v}_{\beta}\right\rangle=\left\langle\mathbf{e}_{j_{1}}, \mathbf{v}_{\beta}\right\rangle .
$$

Hence, $\left[\gamma_{1}, j_{1}\right] \in \Gamma_{\text {srs }}$ which concludes the proof of the first assertion. 
The second assertion is proved as follows. Let $\bar{B}[i, \gamma, j]=\mathcal{J}(i) \cap(\mathcal{J}(j)+\gamma)$ for the moment. By (2.7), we know that

$$
\begin{aligned}
\bar{B}[i, \gamma, j] & =\mathcal{J}(i) \cap(\mathcal{T}(j)+\pi(\gamma)) \\
& =\left(\bigcup_{\sigma\left(i_{1}\right)=p_{1} i_{1}} \mathbf{h} \mathcal{T}\left(i_{1}\right)+\pi \mathbf{l}\left(p_{1}\right)\right) \cap\left(\gamma+\underset{\sigma\left(j_{1}\right)=p_{2} j s_{2}}{\bigcup} \mathbf{h} \mathcal{J}\left(j_{1}\right)+\pi \mathbf{l}\left(p_{2}\right)\right) .
\end{aligned}
$$

It remains to express each intersection $\left(\mathbf{h} \mathcal{T}\left(i_{1}\right)+\pi \mathbf{l}\left(p_{1}\right)\right) \cap\left(\gamma+\mathbf{h} \mathcal{T}\left(j_{1}\right)+\pi \mathbf{l}\left(p_{2}\right)\right)$ in terms of $\bar{B}[\cdot]$. To this matter observe that

$$
\begin{aligned}
\left(\mathbf{h} \mathcal{J}\left(i_{1}\right)+\pi \mathbf{l}\left(p_{1}\right)\right) \cap(\gamma & \left.+\mathbf{h} \mathcal{J}\left(j_{1}\right)+\pi \mathbf{l}\left(p_{2}\right)\right) \\
& =\pi \mathbf{l}\left(p_{1}\right)+\mathbf{h} \bar{B}\left[i_{1}, \mathbf{h}^{-1}\left(\gamma+\pi \mathbf{l}\left(p_{2}\right)-\pi \mathbf{l}\left(p_{1}\right)\right), j_{1}\right] \\
& =\pi \mathbf{l}\left(p_{2}\right)+\gamma+\mathbf{h} \bar{B}\left[j_{1}, \mathbf{h}^{-1}\left(-\gamma-\pi \mathbf{l}\left(p_{2}\right)+\pi \mathbf{l}\left(p_{1}\right)\right), i_{1}\right] .
\end{aligned}
$$

By the definition of the boundary graph, if $\left[i_{1}, \mathbf{h}^{-1}\left(\gamma+\pi \mathbf{l}\left(p_{2}\right)-\pi \mathbf{l}\left(p_{1}\right)\right), j_{1}\right] \in \mathfrak{D}$ and if the associated intersection is nonempty, there is an edge of type 1 from $[i, \gamma, j]$ to $\left[i_{1}, \mathbf{h}^{-1}\left(\gamma+\pi \mathbf{l}\left(p_{2}\right)-\pi \mathbf{l}\left(p_{1}\right)\right), j_{1}\right]$ in the SR-boundary graph. But the node belongs to $\mathfrak{D}$ if and only if $\left\langle\pi \mathbf{l}\left(p_{1}\right), \mathbf{v}_{\beta}\right\rangle \leq\left\langle\mathbf{x}+\pi \mathbf{l}\left(p_{2}\right), \mathbf{v}_{\beta}\right\rangle$, hence, the label of the graph is $\eta=\pi \mathbf{l}\left(p_{1}\right)$.

The second possibility is $\left[j_{1}, \mathbf{h}^{-1}\left(-\gamma-\pi \mathbf{l}\left(p_{2}\right)+\pi \mathbf{l}\left(p_{1}\right)\right), i_{1}\right] \in \mathfrak{D}$. If the associated intersection is nonempty then there is an edge of type 2 from $[i, \gamma, j]$ to $\left[j_{1}, \mathbf{h}^{-1}(-\gamma-\right.$ $\left.\left.\pi \mathbf{l}\left(p_{2}\right)+\pi \mathbf{l}\left(p_{1}\right)\right), i_{1}\right]$ in the graph. The label is then $\eta=\pi \mathbf{l}\left(p_{2}\right)+\gamma$. Inserting this in (7.3) we obtain that

$$
\bar{B}[i, \gamma, j]=\bigcup_{[i, \gamma, j] \stackrel{\eta}{\rightarrow}\left[i_{1}, \gamma_{1}, j_{1}\right] \in \mathscr{G}_{\mathrm{srs}}^{(B)}} \mathbf{h} \bar{B}\left[i_{1}, \gamma_{1}, j_{1}\right]+\eta .
$$

Since the solution of a GIFS is uniquely defined we deduce that $\bar{B}[i, \gamma, j]=B[i, \gamma, j]$ and thus the intersections $\mathcal{T}(i) \cap(\mathcal{T}(j)+\gamma)$ are the solution of the GIFS.

The third assertion follows from the tiling property: we have

$$
\partial \mathcal{T}(i):=\bigcup_{[\gamma, j] \neq[\mathbf{0}, i] \in \Gamma_{\mathrm{srs}}} \mathcal{T}(i) \cap(\mathcal{T}(j)+\gamma) .
$$

Assume that $\gamma \neq \mathbf{0}$ and $\mathcal{T}(i) \cap(\mathcal{T}(j)+\gamma) \neq \varnothing$. Then $\gamma$ satisfies (5.4). From $\gamma \neq \mathbf{0}$ and $[\gamma, j] \in \Gamma_{\text {srs }}$ we deduce $\gamma=\pi(\mathbf{x})$ with $\left\langle\mathbf{x}, \mathbf{v}_{\beta}\right\rangle>0$. Hence $[i, \gamma, j] \in \phi_{\text {srs }}$. Since $\mathcal{J}(i) \cap(\mathcal{J}(j)+\gamma)$ is nonempty, Theorem 5.6 implies that $[i, \gamma, j]$ is a node of the SR-boundary graph. Assume now that $\gamma=\mathbf{0}$. If $i<j$, by the definition of the SR-boundary graph, $\mathcal{T}(i) \cap \mathcal{T}(j) \neq \varnothing$ if and only if $[i, \mathbf{0}, j]$ is a node of the graph. If $i>j$, the set $\mathcal{J}(i) \cap \mathcal{J}(j)$ is nonempty if and only if $[j, \mathbf{0}, i]$ is a node of the graph.

Proof of Proposition 5.17; see also [167]. - Let $[i, \gamma, j]$ be a node of the contact graph. Then $\gamma$ can be written as $\gamma=-\mathbf{h}^{k} \pi\left[\mathbf{l}\left(q_{k}\right)-\mathbf{l}\left(p_{k}\right)\right]-\cdots-\mathbf{h}^{0} \pi\left[\mathbf{l}\left(q_{0}\right)-\mathbf{l}\left(p_{0}\right)\right]+$ $\mathbf{h}^{k+1} \gamma_{0}$ with $\gamma_{0}$ in the set of second coordinates of the finite set $\phi$. Hence, $\gamma$ is uniformly bounded. 
Moreover, $[\gamma, j] \in \Gamma_{\text {srs }}$ by the definition of the contact graph. This yields the result since the first coordinates of the elements of $\Gamma_{\text {srs }}$ form a discrete subset of $\mathbb{H}_{c}$.

Proof of Proposition 5.18. - This result is proved in [167]. The main idea is the following. If there is a walk of length $\ell$ from $[i, \gamma, j]$ to $\left[i_{0}, \gamma_{0}, j_{0}\right]$, then the polygons $\mathcal{J}_{\ell}\left(i_{0}\right)$ and $\mathcal{J}_{\ell}\left(j_{0}\right)+\gamma_{0}$ share a common edge related to $[i, \gamma, j]$.

Proof of Lemma 5.23. - To preserve the set $\left\{\mathbf{e}_{i}, \mathbf{e}_{j}+\gamma_{1}, \mathbf{e}_{k}+\gamma_{2}\right\}$ associated with $\left[i, \gamma_{1}, j, \gamma_{2}, k\right]$ we can act either by translation or by reversing the order. Then $\left\{\mathbf{e}_{i}, \mathbf{e}_{j}+\right.$ $\left.\gamma_{1}, \mathbf{e}_{k}+\gamma_{2}\right\}$ is equal to $\left\{\mathbf{e}_{i}, \mathbf{e}_{k}+\gamma_{2}, \mathbf{e}_{j}+\gamma_{1}\right\}, \gamma_{1}+\left\{\mathbf{e}_{j}, \mathbf{e}_{i}-\gamma_{1}, \mathbf{e}_{k}+\gamma_{2}-\gamma_{1}\right\}, \gamma_{1}+\left\{\mathbf{e}_{j}, \mathbf{e}_{k}+\right.$ $\left.\gamma_{2}-\gamma_{1}, \mathbf{e}_{i}-\gamma_{1}\right\}, \gamma_{2}+\left\{\mathbf{e}_{k}, \mathbf{e}_{i}-\gamma_{2}, \mathbf{e}_{j}+\gamma_{1}-\gamma_{2}\right\}, \gamma_{2}+\left\{\mathbf{e}_{k}, \mathbf{e}_{j}+\gamma_{1}-\gamma_{2}, \mathbf{e}_{i}-\gamma_{2},\right\}$. We deduce that the equivalence class of $\left[i, \gamma_{1}, j, \gamma_{2}, k\right]$ contains the six elements (depending on the elements forming the node, some of them can be equal)

$$
\begin{aligned}
{\left[i, \gamma_{1}, j, \gamma_{2}, k\right] } & \simeq_{t}\left[i, \gamma_{2}, k, \gamma_{1}, j\right] \simeq_{t}\left[j,-\gamma_{1}, i, \gamma_{2}-\gamma_{1}, k\right] \\
& \simeq_{t}\left[j, \gamma_{2}-\gamma_{1}, k,-\gamma_{1}, i\right] \simeq_{t}\left[k,-\gamma_{2}, i, \gamma_{1}-\gamma_{2}, j\right] \simeq_{t}\left[k, \gamma_{1}-\gamma_{2}, j,-\gamma_{2}, i\right] .
\end{aligned}
$$

In order to choose a unique candidate, we set $\gamma_{0}=\mathbf{0}$ and we denote $a_{0}=i, a_{1}=j$, $a_{2}=k$. For each $\gamma_{i}$ there exists $\mathbf{x}_{i} \in \mathbb{Z}^{n}$ such that $\gamma_{i}=\pi\left(\mathbf{x}_{i}\right)$. By $(2.5),\left\langle\mathbf{x}_{i}, \mathbf{v}_{\beta}\right\rangle$ depends only on $\gamma_{i}$. We choose a permutation $\mu$ on $\{0,1,2\}$ such that the quantities $\left\langle\mathbf{x}_{i}, \mathbf{v}_{\beta}\right\rangle$ are ordered and, when there is an ambiguity, the associated letters $a_{i}$ are also ordered, i.e.,

$$
\begin{aligned}
& \left\langle\mathbf{x}_{\mu(0)}, \mathbf{v}_{\beta}\right\rangle \leq\left\langle\mathbf{x}_{\mu(1)}, \mathbf{v}_{\beta}\right\rangle \leq\left\langle\mathbf{x}_{\mu(2)}, \mathbf{v}_{\beta}\right\rangle, \\
& \left\langle\mathbf{x}_{\mu(\alpha)}, \mathbf{v}_{\beta}\right\rangle=\left\langle\mathbf{x}_{\mu(\beta)}, \mathbf{v}_{\beta}\right\rangle \Longrightarrow a_{\mu(\alpha)}<a_{\mu(\beta)} .
\end{aligned}
$$

A unique permutation satisfies these conditions. The unique node equivalent to $\left[i, \gamma_{1}, j, \gamma_{2}, k\right]$ in $\overline{\mathfrak{T}}$ is then obtained by translating the smallest quantity $\left\langle\mathbf{x}_{\mu(0)}, \mathbf{v}_{\beta}\right\rangle$ to zero, that is, $\left[a_{\mu(0)}, \gamma_{\mu(1)}-\gamma_{\mu(0)}, a_{\mu(1)}, \gamma_{\mu(2)}-\gamma_{\mu(0)}, a_{\mu(2)}\right]$.

Proof of Theorem 5.25. - The proof of the finiteness of the graph $\mathscr{G}^{(T)}$ is the same as the proof of Proposition 5.5. Consider a node $\left[i, \gamma_{1}, j, \gamma_{2}, k\right]$ that satisfies (1) and (3). Then $\gamma_{1}$ and $\gamma_{2}$ can be expanded as

$$
\gamma_{\ell}=\pi\left(\mathbf{x}_{\ell}\right)=\pi\left( \pm \mathbf{M}^{-1} \mathbf{l}\left(p_{\ell}^{(k)}\right) \pm \cdots \pm \mathbf{M}^{-k} \mathbf{l}\left(p_{\ell}^{(1)}\right) \pm \mathbf{M}^{-k-1} \mathbf{l}\left(p_{\ell}^{(0)}\right) \pm \mathbf{M}^{-k-1} \mathbf{x}_{\ell}^{(0)}\right) .
$$

By the argument used in Proposition 5.5, $\gamma_{1}$ and $\gamma_{2}$ are contained in a finite set, so that the triple point graph is finite.

We mimic the proof of Theorem 5.6 to demonstrate the assertion about intersections of tiles. Consider a walk $\left(\left[i^{(\nu)}, \gamma_{1}^{(\nu)}, j^{(\nu)}, \gamma_{2}^{(\nu)}, k^{(\nu)}\right]\right)_{\nu \geq 0}$ in the triple point graph. By the definition of the edges, there exist three walks in the prefix-suffix graph starting in $i^{(0)}, j^{(0)}$ and $k^{(0)}$, respectively, such that

$$
\begin{aligned}
\gamma_{1}^{(0)} & =-\sum_{\nu \geq 0} \mathbf{h}^{\nu} \pi \mathbf{l}\left(p_{1}^{(\nu)}\right)+\sum_{\nu \geq 0} \mathbf{h}^{\nu} \pi \mathbf{l}\left(p_{0}^{(\nu)}\right) \\
\gamma_{2}^{(0)} & =-\sum_{\nu \geq 0} \mathbf{h}^{\nu} \pi \mathbf{l}\left(p_{2}^{(\nu)}\right)+\sum_{\nu \geq 0} \mathbf{h}^{\nu} \pi \mathbf{l}\left(p_{0}^{(\nu)}\right) .
\end{aligned}
$$


We deduce that $\mathcal{T}\left(j^{(0)}\right)+\gamma_{1}^{(0)}$ and $\mathcal{T}\left(k^{(0)}\right)+\gamma_{2}^{(0)}$ both contain the point $\sum_{\nu \geq 0} \mathbf{h}^{\nu} \pi \mathbf{l}\left(p_{0}^{(\nu)}\right)$ that belongs to $\mathcal{T}\left(i^{(0)}\right)$. This yields $\mathcal{T}\left(i^{(0)}\right) \cap\left(\mathcal{J}\left(j^{(0)}\right)+\gamma_{1}^{(0)}\right) \cap\left(\mathcal{T}\left(k^{(0)}\right)+\gamma_{2}^{(0)}\right) \neq \varnothing$.

Conversely, assume that $\mathcal{T}(i) \cap\left(\mathcal{T}(j)+\gamma_{1}\right) \cap\left(\mathcal{T}(k)+\gamma_{2}\right) \neq \varnothing$, with $\left[i, \gamma_{1}, j, \gamma_{2}, k\right] \in \mathfrak{T}$ and $\left[\gamma_{1}, j\right] \in \Gamma_{\text {srs }},\left[\gamma_{2}, k\right] \in \Gamma_{\text {srs }}$. Set $\left[i^{(0)}, \gamma_{1}^{(0)}, j^{(0)}, \gamma_{2}^{(0)}, k^{(0)}\right]=\varphi_{\mathfrak{T}}\left[i, \gamma_{1}, j, \gamma_{2}, k\right]$. By the definition of $\varphi_{\overline{\mathfrak{T}}}$, we also have $\left[\gamma_{1}^{(0)}, j^{(0)}\right] \in \Gamma_{\text {srs }}$ and $\left[\gamma_{2}^{(0)}, k^{(0)}\right] \in \Gamma_{\text {srs }}$.

Since the intersection is nonempty we exhibit three walks in the prefix-suffix graph (starting in $i^{(0)}, j^{(0)}, k^{(0)}$, respectively) with $\gamma_{1}^{(0)}=-\sum_{\nu \geq 0} \mathbf{h}^{\nu} \pi \mathbf{l}\left(p_{1}^{(\nu)}\right)+$ $\sum_{\nu \geq 0} \mathbf{h}^{\nu} \pi \mathbf{l}\left(p_{0}^{(\nu)}\right)$ and $\gamma_{2}^{(0)}=-\sum_{\nu \geq 0} \mathbf{h}^{\nu} \pi \mathbf{l}\left(p_{2}^{(\nu)}\right)+\sum_{\nu \geq 0} \mathbf{h}^{\nu} \pi \mathbf{l}\left(p_{0}^{(\nu)}\right)$. We define $\gamma_{\ell}^{(\rho)}=-\sum_{\nu \geq 0} \mathbf{h}^{\nu} \pi \mathbf{l}\left(p_{\ell}^{(\nu+\rho)}\right)+\sum_{\nu \geq 0} \mathbf{h}^{\nu} \pi \mathbf{l}\left(p_{0}^{(\nu+\rho)}\right)$ for $\ell \in\{1,2\}$ and $\rho \in \mathbb{N}$.

Then the nodes $\varphi_{\overline{\mathfrak{T}}}\left[i^{(\nu)}, \gamma_{1}^{(\nu)}, j^{(\nu)}, \gamma_{2}^{(\nu)}, k^{(\nu)}\right]$ belong to $\overline{\mathfrak{T}}$; they satisfy condition (1) and they give rise to an infinite walk in the triple point graph.

As in the proof of Theorem 5.7, we deduce a GIFS equation of the triple intersections, in particular,

$$
T\left[i, \gamma_{1}, j, \gamma_{2}, k\right]=\underset{\left[i, \gamma_{1}, j, \gamma_{2}, k\right] \stackrel{\eta}{\rightarrow}\left[i^{\prime}, \gamma_{1}^{\prime}, j^{\prime}, \gamma_{2}^{\prime}, k^{\prime}\right] \in \mathscr{G}^{(T)}}{\bigcup} \mathbf{h} T\left[i^{\prime}, \gamma_{1}^{\prime}, j^{\prime}, \gamma_{2}^{\prime}, k^{\prime}\right]+\eta .
$$

This implies that any point in $T\left[i, \gamma_{1}, j, \gamma_{2}, k\right]$ can be expanded by using the labels of a walk of the triple point graph starting from $\left[i, \gamma_{1}, j, \gamma_{2}, k\right]$.

Proof of Proposition 5.33. - The proof is given in the case of the SR-boundary graph. The cases of triple or quadruple point graphs are similar.

Assume that a point $\zeta$ corresponds to infinitely many different walks starting from a node $N_{0}=\left[i^{(0)}, \gamma^{(0)}, j^{(0)}\right]$ of the SR-boundary graph. We denote these walks by $w_{k}: N_{0} \stackrel{\eta_{k}^{(1)}}{\longrightarrow} N_{k}^{(1)} \stackrel{\eta_{k}^{(2)}}{\longrightarrow} N_{k}^{(2)} \stackrel{\eta_{k}^{(3)}}{\longrightarrow} \cdots$ with $N_{k}^{(l)}=\left[i_{k}^{(l)}, \gamma_{k}^{(l)}, j_{k}^{(l)}\right] . \quad$ By the local finiteness of the self-replicating multiple tiling (see Remark 3.8), there exists a positive integer $P$ such that each point of $\mathbb{H}_{c}$ is covered at most $P$ times by the tiles of this multiple tiling. Since the walks $w_{k}$ are all distinct, there exists a positive integer $\nu$ and $P+1$ walks, say $w_{1}, \ldots, w_{P+1}$, such that the prefixes of length $\nu$ of $w_{1}, \ldots, w_{P+1}$ are pairwise distinct. From Theorem 5.7 we have that

$$
\mathcal{T}\left(i^{(0)}\right) \cap\left(\mathcal{T}\left(j^{(0)}\right)+\gamma^{(0)}\right)=\bigcup \mathbf{h}^{\nu}\left(\mathcal{T}\left(i^{(\nu)}\right) \cap\left(\mathcal{T}\left(j^{(\nu)}\right)+\gamma^{(\nu)}\right)\right)+\eta^{(1)}+\mathbf{h} \eta^{(2)}+\cdots+\mathbf{h}^{\nu-1} \eta^{(\nu)},
$$

where the union is extended over all walks

$$
N^{(0)} \stackrel{\eta^{(1)}}{\longrightarrow} N^{(1)} \stackrel{\eta^{(2)}}{\longrightarrow} N^{(2)} \stackrel{\eta^{(3)}}{\longrightarrow} \cdots \stackrel{\eta^{(\nu)}}{\longrightarrow} N^{(\nu)}
$$

of length $\nu$ in the SR-boundary graph. In particular, the tiles $\mathbf{h}^{-\nu} \eta_{k}^{(1)}+\mathbf{h}^{-\nu+1} \eta_{k}^{(2)}+$ $\cdots+\mathbf{h}^{-1} \eta_{k}^{(\nu)}+\mathcal{T}\left(i_{k}^{(\nu)}\right)$ are pairwise distinct tiles of the self-replicating multiple tiling. However, by Corollary 5.9, the point $\zeta$ belongs to each of these $P+1$ tiles, which is impossible by the choice of $P$, a contradiction.

Consider now a walk $w: N^{(0)} \stackrel{\eta^{(1)}}{\longrightarrow} N^{(1)} \stackrel{\eta^{(2)}}{\longrightarrow} N^{(2)} \stackrel{\eta^{(3)}}{\longrightarrow} \cdots$ in the SR-boundary graph that is not ultimately periodic. Since the SR-boundary graph is finite, two 
nodes in this walk are equal, i.e., there exist positive integers $\nu$ and $p$ such that $N^{(\nu)}=N^{(\nu+p)}$. Thus $w$ contains a piece

$$
v: N^{(\nu)} \stackrel{\eta^{(\nu+1)}}{\longrightarrow} \cdots \stackrel{\eta^{(\nu+p)}}{\longrightarrow} N^{(\nu+p)} .
$$

Hence, there is a prefix $w_{1}$ of length $\nu$ and an infinite suffix $w_{2}$ such that $w=w_{1} v w_{2}$. Denote the $r$-fold repetition of the walk $v$ by $v^{r}$. Since $w$ is not ultimately periodic, the walks

$$
w_{k}=w_{1} v^{k} w_{2} \quad(k \geq 1)
$$

are infinitely many different walks starting at $N^{(0)}$.

Consequently, if only a finite number of infinite walks lead away from a given node $\mathcal{N}$ of the SR-boundary graph, these walks all have to be ultimately periodic. This implies that the corresponding points can be calculated exactly from the formula $\zeta=\sum_{\nu \geq 0} \mathbf{h}^{\nu} \eta^{(\nu)}$, since the ultimate periodicity of the sequence $\left(\eta^{(\nu)}\right)_{\nu \geq 1}$ makes this formula to a "rational function" in $\mathbf{h}$.

\subsection{Details for the quadruple point graph}

To deal with quadruple intersections, we reduce the set of all possible intersections defined in (5.16).

$\overline{\mathfrak{Q}}=\left\{\begin{array}{l|l}{\left[i, \gamma_{1}, j, \gamma_{2}, k, \gamma_{3}, l\right] \in \mathfrak{Q}} & \begin{array}{l}0 \leq\left\langle\mathbf{x}_{1}, \mathbf{v}_{\beta}\right\rangle \leq\left\langle\mathbf{x}_{2}, \mathbf{v}_{\beta}\right\rangle \leq\left\langle\mathbf{x}_{3}, \mathbf{v}_{\beta}\right\rangle \\ \gamma_{1}=\pi\left(\mathbf{x}_{1}\right), \gamma_{2}=\pi\left(\mathbf{x}_{2}\right), \gamma_{3}=\pi\left(\mathbf{x}_{3}\right) ;\end{array} \\ \mathbf{x}_{1}, \mathbf{x}_{2}, \mathbf{x}_{3} \in \mathbb{Z}^{n} & \begin{array}{l}\text { if } \gamma_{1}=0 \text { then } i<j \\ \text { if } \gamma_{2}=\gamma_{1} \text { then } j<k \\ \text { if } \gamma_{3}=\gamma_{2} \text { then } k<l\end{array}\end{array}\right\}$.

As for the triple point graph, the set $\overline{\mathfrak{Q}}$ provides a unique representant of intersections between four tiles.

LEMMA 7.1. - Let us define the following equivalence relation on $\mathfrak{Q}$. 4-tuples are equivalent, i.e.,

$$
\left.\left[i, \gamma_{1}, j, \gamma_{2}, k, \gamma_{3}, l\right]\right) \simeq_{q}\left[i^{\prime}, \gamma_{1}^{\prime}, j^{\prime}, \gamma_{2}^{\prime}, k^{\prime}, \gamma_{3}^{\prime}, l^{\prime}\right]
$$

if and only if the sets $\left\{\mathbf{e}_{i}, \mathbf{e}_{j}+\gamma_{1}, \mathbf{e}_{k}+\gamma_{2}, \mathbf{e}_{l}+\gamma_{3}\right\}$ and $\left\{\mathbf{e}_{i^{\prime}}, \mathbf{e}_{j^{\prime}}+\gamma_{1}^{\prime}, \mathbf{e}_{k^{\prime}}+\gamma_{2}^{\prime}, \mathbf{e}_{l^{\prime}}+\gamma_{3}^{\prime}\right\}$ are equal up to a translation. The set $\overline{\mathfrak{Q}}$ is a quotient set for the equivalence relation $\simeq_{q}$, i.e., for every $\left[i, \gamma_{1}, j, \gamma_{2}, k, \gamma_{3}, l\right]$, there exists a unique element in $\overline{\mathfrak{Q}}$, denoted by $\varphi_{\overline{\mathfrak{Q}}}\left(\left[i, \gamma_{1}, j, \gamma_{2}, k, \gamma_{3}, l\right]\right) \in \overline{\mathfrak{Q}}$ such that $\left[i, \gamma_{1}, j, \gamma_{2}, k, \gamma_{3}, l\right] \simeq_{q} \varphi_{\overline{\mathfrak{Q}}}\left(\left[i, \gamma_{1}, j, \gamma_{2}, k, \gamma_{3}, l\right]\right)$.

Proof. - As in the case of intersections between three tiles, we reorder the labels of tiles and translate appropriately. We deduce that there are a priori 24 sets being 
equivalent to $\left[i, \gamma_{1}, j, \gamma_{2}, k, \gamma_{3}, l\right]$ (some of them can be equal), i.e.,

$\left[i, \gamma_{1}, j, \gamma_{2}, k, \gamma_{3}, l\right]$

$\simeq_{q}\left[i, \gamma_{2}, k, \gamma_{1}, j, \gamma_{3}, l\right]$ and all permutations between $j, k, l$

$\simeq_{q}\left[j,-\gamma_{1}, i, \gamma_{2}-\gamma_{1}, k, \gamma_{3}-\gamma_{1}, l\right]$ and all permutations between $i, k, l$

$\simeq_{q}\left[k,-\gamma_{2}, i, \gamma_{1}-\gamma_{2}, j, \gamma_{3}-\gamma_{2}, l\right]$ and all permutations between $i, j, l$

$\simeq_{q}\left[l,-\gamma_{3}, i, \gamma_{1}-\gamma_{3}, j, \gamma_{2}-\gamma_{3}, k\right]$ and all permutations between $i, j, k$.

Selecting the unique representant contained in $\overline{\mathfrak{Q}}$ is now done in a similar way as for the triple point graph (see the proof of Lemma 5.23).

As for triple intersections, we deduce that if $Q\left[i, \gamma_{1}, j, \gamma_{2}, k, \gamma_{3}, l\right]$ denotes the intersection between the four associated tiles, i.e.,

$$
\left.Q\left[i, \gamma_{1}, j, \gamma_{2}, k, \gamma_{3}, l\right]=\mathcal{J}(i) \cap\left(\mathcal{J}(j)+\gamma_{1}\right) \cap\left(\mathcal{J}(k)+\gamma_{2}\right) \cap\left(\mathcal{T}(l)+\gamma_{3}\right)\right),
$$

then $Q\left[i, \gamma_{1}, j, \gamma_{2}, k, \gamma_{3}, l\right]$ and $Q \varphi_{\overline{\mathfrak{Q}}}\left[i, \gamma_{1}, j, \gamma_{2}, k, \gamma_{3}, l\right]$ are equal up to a translation vector.

The quadruple point graph is then defined as follows.

DEFinition 7.2 (Quadruple point graph). - The quadruple point graph of a primitive unit Pisot substitution $\sigma$ is denoted by $\mathscr{G}^{(Q)}$. It the largest ${ }^{(1)}$ graph such that

1. $\left[i, \gamma_{1}, j, \gamma_{2}, k, \gamma_{3}, l\right] \in \overline{\mathfrak{Q}}$ is a node of $\mathscr{G}^{(Q)}$ if

$$
\max \left\{\left\|\gamma_{1}\right\|,\left\|\gamma_{2}\right\|,\left\|\gamma_{3}\right\|\right\} \leq \frac{2 \max \{\|\pi \mathbf{l}(p)\| ;(p, a, s) \in \mathscr{P}\}}{1-\max \left\{\left|\beta^{(\ell)}\right| ; \ell=2, \ldots, d\right\}} .
$$

2. There is a directed edge from $\left[i, \gamma_{1}, j, \gamma_{2}, k, \gamma_{3}, l\right]$ to $\left[i^{\prime}, \gamma_{1}^{\prime}, j^{\prime}, \gamma_{2}^{\prime}, k^{\prime}, \gamma_{3}^{\prime}, l^{\prime}\right]$ if and only if there exists $\left[\bar{i}, \overline{\gamma_{1}}, \bar{j}, \overline{\gamma_{2}}, \bar{k}, \overline{\gamma_{3}}, \bar{l}\right] \in \mathfrak{Q}$ and $\left(p_{0}, a_{0}, s_{0}\right),\left(p_{1}, a_{1}, s_{1}\right)$, $\left(p_{2}, a_{2}, s_{2}\right),\left(p_{3}, a_{3}, s_{3}\right)$ such that

$$
\left\{\begin{array}{l}
{\left[i^{\prime}, \gamma_{1}^{\prime}, j^{\prime}, \gamma_{2}^{\prime}, k^{\prime}, \gamma_{3}^{\prime}, l^{\prime}\right]=\Phi \overline{\mathfrak{Q}}\left[\bar{i}, \overline{\gamma_{1}}, \bar{j}, \overline{\gamma_{2}}, \bar{k}, \overline{\gamma_{3}}, \bar{l}\right]} \\
a_{0}=i \text { and } p_{0} a_{0} s_{0}=\sigma(\bar{i}), \\
a_{1}=j \text { and } p_{1} a_{1} s_{1}=\sigma(\bar{j}) \\
a_{2}=k \text { and } p_{2} a_{2} s_{2}=\sigma(\bar{k}) \\
a_{3}=l \text { and } p_{3} a_{3} s_{3}=\sigma(\bar{l}) \\
\mathbf{h} \overline{\gamma_{1}}=\gamma_{1}+\pi \mathbf{l}\left(p_{1}\right)-\pi \mathbf{l}\left(p_{0}\right) \\
\mathbf{h} \overline{\gamma_{2}}=\gamma_{2}+\pi \mathbf{l}\left(p_{2}\right)-\pi \mathbf{l}\left(p_{0}\right) \\
\mathbf{h} \overline{\gamma_{3}}=\gamma_{3}+\pi \mathbf{l}\left(p_{3}\right)-\pi \mathbf{l}\left(p_{0}\right)
\end{array}\right.
$$

This edge is labeled by $\eta \in\left\{\pi \mathbf{l}\left(p_{0}\right), \pi \mathbf{l}\left(p_{1}\right)+\gamma_{1}, \pi \mathbf{l}\left(p_{2}\right)+\gamma_{2},, \pi \mathbf{l}\left(p_{3}\right)+\gamma_{3}\right\}$ such that $\left\langle\mathbf{x}, \mathbf{v}_{\beta}\right\rangle=\min \left\{\left\langle\mathbf{l}\left(p_{0}\right), \mathbf{v}_{\beta}\right\rangle,\left\langle\mathbf{l}\left(p_{1}\right)+\mathbf{x}_{1}, \mathbf{v}_{\beta}\right\rangle,\left\langle\mathbf{l}\left(p_{2}\right)+\mathbf{x}_{2}, \mathbf{v}_{\beta}\right\rangle,\left\langle\mathbf{l}\left(p_{3}\right)+\mathbf{x}_{3}, \mathbf{v}_{\beta}\right\rangle\right\}$. Here $\pi(\mathbf{x})=\eta$ and $\pi\left(\mathbf{x}_{\ell}\right)=\gamma_{\ell}$, where $\mathbf{x}, \mathbf{x}_{\ell} \in \mathbb{Z}^{n}(\ell \in\{1,2,3\})$.

(1) The meaning of "largest" is explained in Definition 5.1. 
3. Every node belongs to an infinite walk starting from a node $\left[i, \gamma_{1}, j, \gamma_{2}, k, \gamma_{3}, l\right]$ such that $\left[\gamma_{1}, j\right] \in \Gamma_{\mathrm{srs}},\left[\gamma_{2}, k\right] \in \Gamma_{\mathrm{srs}}$ and $\left[\gamma_{3}, l\right] \in \Gamma_{\mathrm{srs}}$.

With a treatment similar to the one in the setting of the triple point graph, we prove that this graph is finite and identifies quadruple points in the self-replicating multiple tiling.

Proof of Theorem 5.30. - The proof is exactly the same as the proof of the triple point case in Theorem 5.25. The finiteness of the graph $\mathscr{G}^{(Q)}$ is deduced from Condition (7.4). Moreover, if $\left[i, \gamma_{1}, j, \gamma_{2}, k, \gamma_{3}, l\right] \in \overline{\mathfrak{Q}}$ is a node of the quadruple point graph, we can express a point that lies at the intersection of the four tiles as a power series.

The algorithmic construction of the quadruple point graph runs along the same lines as for the triple point graph.

REMARK 7.3. - It is now clear how to define $m$-tuple graphs for $m \geq 5$ also. 



\section{CHAPTER 8}

\section{PERSPECTIVES}

In the present monograph we show many topological results for central tiles of primitive unit Pisot substitutions. The main objects used in our study are different kinds of graphs. We consider these graphs as powerful tools that can be used in order to derive various results on substitutions, their associated Dumont-Thomas numeration as well as their tiles and tilings. In this chapter we want to give some perspectives for possible future work in this direction.

\subsection{Topology}

A first direction of research consists in pursuing the study of the topological structure of central tiles corresponding to primitive unit Pisot substitutions. In the examples we considered throughout this monograph, we exhibited compact subsets of the plane whose fundamental groups are uncountable and not free. Such sets are "pathological" from a topological point of view. Getting more details on the structure of the fundamental group in such cases is a first task for future research. The fact that a central tile has uncountable fundamental group has the consequence that it is not locally simply connected. Fundamental groups of such spaces are studied in the literature. The easiest example of a space with this property is the so-called Hawaiian Earring (see for instance [160]). Its fundamental group is studied in great detail in [58], where it is described by means of words. Another example for a not locally simply connected space whose fundamental group has been described is the Sierpiński gasket (see [16]). Structural results on fundamental groups of not locally simply connected spaces can be found for instance in $[\mathbf{6 5}, \mathbf{7 9}]$. The main difficulty in getting results on the structure of the fundamental group of a central tile in the plane comes from the fact that its topological dimension is 2 . All the spaces that have been investigated so far have topological dimension 1. And this fact is heavily used in the various proofs.

Other questions on the topology of central tiles are related to components of the interior of a central tile. There are several connected Rauzy fractals whose interior 
is disconnected. It would be interesting to get results on the structure of the components of their interior. Can the closure of a component of the interior be represented as the solution of a certain GIFS (see [117], where this question has been studied for an example of a self-similar lattice tile)? Is it homeomorphic to a closed disk? Similar questions have been investigated in the setting of self-affine tiles (see for instance $[\mathbf{1 2 3}, \mathbf{1 3 2}, \mathbf{1 3 3}, \mathbf{1 3 4}])$. However, the fact that the central tile and its subtiles are the solution of GIFS makes things much more complicated. In view of Torhorst's theorem (see [107, §61, II, Theorem 4]) and the tiling property, the disklikeness of the components of the interior of a central tile is linked with the question whether this tile has cut points or not. Therefore, a criterion for cut points in terms of our graphs would be a desirable tool in this circle of problems.

Another direction is concerned with topological properties of higher dimensional central tiles. Many of the topological properties we obtained in the present monograph are obtained for substitutions whose corresponding central tiles are subsets of the plane. The reason for restricting to this case is that we based our proofs on separation properties of the Euclidean plane; such properties (like the Jordan curve theorem) are no longer satisfied in higher dimensions. However, the definition of boundary or contact graphs is independent from the dimension that is considered (using these graphs, neighbors of a single example of a three dimensional central tile are calculated in [78]). A possible topic for further research is to obtain for instance criteria for simple-connectivity or ballikeness of a central tile in dimension 3. These criteria should be based on the GIFS structure of this tile so that they can be checked by using contact and boundary graphs. One theorem which could be useful in this context is the Moore-van Kampen-Zippin Theorem (see for instance [103]). This theorem gives a characterization of a sphere $\mathbb{S}^{2}$ in terms of cut sets. For the case of boundaries of three dimensional central tiles the structure of cut sets can probably be checked with help of our graphs in order to exhibit a three dimensional central tile whose boundary is homeomorphic to a sphere.

\subsection{Number theory}

As mentioned in the introduction, one historical reason for introducing central tiles refers to the study of numeration systems [166]. Let us detail now the potential applications of our methods in this field.

When studying a numeration system, basic problems one has to settle are for instance to characterize the set of admissible expansions and to identify which numbers have a finite, an eventually periodic or a purely periodic expansion. For numeration systems with integer base or continued fractions, these questions are completely solved. For beta-numeration, however, the question of purely periodic expansions is not clearly understood. A classical method to study numbers with a purely periodic expansion is to build a suitable natural extension of the dynamics; in this natural extension periodic expansion correspond to finite orbits and thus can be identified. In 
this setting, the central tile plays a crucial role in the context of beta-numeration. Indeed, Ito and Rao [100] proved that a suitable natural extension is obtained by adding an expanding component to the central tile of the corresponding beta-substitution. This geometrical characterization builds a bridge between number theory and geometry. As an example, based on our geometrical characterization, the geometric property (F) implies that each rational number sufficiently near to zero has a purely periodic beta-expansion in the primitive unit Pisot case $[\mathbf{1 1}, \mathbf{1 0 0}]$. In other words, the geometry of the central tile (in particular, the question whether $\mathbf{0}$ is an inner point or not) relates to properties of beta-numeration (note that the behavior of rational numbers with respect to purely periodic beta-expansions is far from random). An interesting question is now: does connectivity, or disklikeness of the central tile have an influence on the structure of the subset of real numbers with a purely periodic expansion?

To go further, we know that when $\beta$ is still a Pisot number but not a unit, a suitable natural extension is not built from the central tile itself but it requires additional $p$-adic components (see $[\mathbf{1 1}, \mathbf{4 6}]$ ). In spite of that, the construction of the natural extension remains very similar to the unit case and boundary graphs can be defined as well in this situation. However, the previous relation between the geometric property $(\mathrm{F})$ and purely periodic expansions of all rationals near to zero becomes false: The geometric property $(\mathrm{F})$ still implies that $\mathbf{0}$ is an inner point of the central tile, but for instance for $\beta=2+\sqrt{7}$, there exists a sequence of rationals converging to zero and having nonpurely periodic expansion. The proof of this fact is based on boundary graphs (see [11]). It would be useful to have good topological conditions characterizing purely periodic expansions near zero in the non-unit case.

We also want to gain a better understanding of the expansion of some specific points. For instance, what is the expansion of the infimum of all rationals with a nonpurely periodic expansion? Computations showed for $\beta^{3}=\beta+1$ that this number is slightly smaller than $2 / 3[\mathbf{1 9}]$. In $[\mathbf{3}]$ it is shown that this number is irrational. Interesting questions are whether it belongs to $\mathbb{Q}(\beta)$ or whether it is transcendental. The technical tool needed in order to answer questions of this kind is to describe properly the intersection of a fractal curve with a line. Another example of interest in connection with diophantine approximation (see e.g. $[\mathbf{9 4}, \mathbf{9 6}]$ ) is to compute the largest ball centered at zero which is contained in the central tile. Here the problem we have to settle is to describe and compute the exact intersection between a fractal curve and a circle.

In order to check the associativity of the so-called Fibonacci multiplication and its generalizations (see for instance [41]) one needs to consider the set $\mathscr{T} \cdot \mathcal{T}=\{\mathbf{x y} ; \mathbf{x}, \mathbf{y} \in$ $\mathcal{T}\}$ and its boundary (here $\mathcal{T} \subset \mathbb{C}$ denotes e.g. the tile associated to the Tribonacci substitution). Also in this context versions of our graphs might be useful to get further results.

Another topic is to generalize those considerations to other numeration systems. We present two types of dynamical systems that are closely related to central tiles and deserve more specific investigation that is related to the graphs we introduced. 
The first class of dynamical systems are shift radix systems (see [12]). We recall their definition. For $\mathbf{r} \in \mathbb{R}^{d}$ define the function

$$
\tau_{\mathbf{r}}: \mathbb{Z}^{d} \rightarrow \mathbb{Z}^{d}, \quad \mathbf{z}=\left(z_{0}, \ldots, z_{d-1}\right) \mapsto\left(z_{1}, \ldots, z_{d-1},-\lfloor\mathbf{r z}\rfloor\right),
$$

where $\mathbf{r z}$ is the scalar product of the vectors $\mathbf{r}$ and $\mathbf{z}$. The mapping $\tau_{\mathbf{r}}$ is called a shift radix system $(S R S)$ if for each $\mathbf{z} \in \mathbb{Z}^{d}$ there exists $k \in \mathbb{N}$ such that $\tau_{\mathbf{r}}^{k}(\mathbf{z})=\mathbf{0}$. These dynamical systems form a generalization of beta-numeration (see [12, Section 2]). Their arithmetic properties have been studied thoroughly (see for instance $[\mathbf{1 2}, \mathbf{1 3}$, 14, 15]). One can also attach central tiles to SRS, however, in general they are not the solution of a GIFS. For this reason the topology as well as the tiling properties of these tiles are much harder to study. First results on these tiles are contained in [47]. In particular, it is shown in this paper that they give rise to multiple tilings for almost every parameter $\mathbf{r}$ contained in a certain compact subset $\mathscr{D}_{d}$ of $\mathbb{R}^{d}$ (the set $\mathscr{D}_{d}$ is related to the Schur-Cohn region defined in terms of coefficients of contracting polynomials). A next step here would be to describe the boundary of these tiles by means of (possibly infinite) graphs related to the SR-boundary graph. Moreover, connectivity properties of such tiles deserve to be investigated. As the parameter $\mathbf{r}$ varies in a compact subset of $\mathbb{R}^{d}$, this would lead to new types of Mandelbrot sets.

The second family of dynamical systems related to central tiles is Dumont-Thomas numeration $[\mathbf{7 4}, \mathbf{7 5}, \mathbf{7 6}]$; as detailed in Section 2.6, this kind of numeration can be seen as an extension of beta-numeration to the substitutive case $[\mathbf{3 3}, \mathbf{4 5}]$. We expect that Dumont-Thomas numeration can be studied with help of our graphs. The SRboundary as well as the SR-contact graph should be related to addition of certain quantities to h-ary representations (see 2.8) which are conjugate to $(\sigma, i)$-expansions of Dumont-Thomas numeration (see Theorem 2.15). In particular, we expect that these graphs act as odometers for these numeration systems. For beta-numeration and numeration systems related to full shifts a correspondence between boundary graphs and addition automata has been observed for instance in $[\mathbf{4 0 , 1 4 9 ]}$. Moreover, in the framework of beta-numeration the structure of periods occurring in beta-expansions with respect to quadratic Pisot numbers as bases has been investigated (see [138]). Our graphs might be the appropriate tools to extend these considerations to DumontThomas numeration.

\subsection{Invariants in dynamics and geometry}

Another independent historical reason for the introduction of central tiles is the study of dynamical systems. This story started with Rauzy [141] who aimed at building an example of a domain exchange in $\mathbb{R}^{2}$ that generalizes the theory on interval exchange transformations $[\mathbf{1 0 4}, \mathbf{1 6 8}]$. Thereby he constructed the classical Rauzy fractal which is depicted in Figure 1.1. It is equal to the central tile of the Tribonacci substitution and its subtiles generate a Markov partition for the action of the incidence matrix of the Tribonacci substitution on a torus $[\mathbf{9 8 , ~ 1 3 6 ] . ~ N o t i c e ~ t h a t ~ t h i s ~ M a r k o v ~}$ partition is the same as the natural extension mentioned above to exhibit purely 
periodic beta-expansions. This story can be revisited in the framework of hyperbolic attractors as detailed in [35]: from the considerations in $[\mathbf{2 2}, \mathbf{3 6}, \mathbf{1 6 9}]$ it follows that every orientable hyperbolic one-dimensional attractor is either a substitution tiling space or a classical solenoid. It is thus proposed to study the topology of tiling spaces in order to understand the flow acting on an arc component of the attractor. The relation with central tiles is that the Markov partition built from the central tile is also a suitable geometric representation of the substitution tiling space. The substitutive dynamical system appears here as an expanding foliation in the space tiling [36]. In [35], the authors prove that branch loci in tiling spaces are invariant for homeomorphic tiling spaces. A natural question is then how to characterize branch loci in the central tile as intersections of tiles and to derive from SR-boundary or SR-contact graphs an explicit criterion for orientable hyperbolic one-dimensional attractors.

This question can be seen from a more general point of view. Indeed, branch loci or other topological invariants determine the substitutive tiling flow, where the Rauzy fractal represents the substitutive dynamical system, or, in other words, a section of the substitutive tiling flow. Then, if branch loci are invariants of the full tiling flow, they should appear in each section of the flow, hence, in several Rauzy fractals. A natural question then consists in characterizing substitutions that produce (i.e, that are sections of) the same tiling flow. However, several pictures indicate that the central tiles for substitutions that are conjugate to each other look globally the same (see [27]). Therefore, a related question is to check which topological properties are invariant under the action of invertible substitutions. We assume that some (local) topological properties of central tiles are invariant under conjugacy, such as the existence of (local) cut points or connectivity. To go further in that direction, we need both to propose a topological criterion to characterize the existence of cut points (as already mentioned in Section 8.1) and to make explicit how boundary and contact graphs change when applying an invertible substitution to a given substitution.

A motivation for this is to generate new invariants for automorphisms of a free group. It is obvious that any substitution naturally extends to an endomorphism of a free group, and those that extend to an automorphism are called invertible substitutions. Although invertibility does not play a significant role in the case of substitutions, it does in the case of morphisms of a free group mainly because most geometric constructions lead to automorphisms. A specific case is given by homeomorphisms of orientable surfaces with nonempty boundary: the homeomorphism of the surface can be coded into an automorphism of the homotopy group of the surface, which is called geometrical. Notice that even if all automorphisms of the free group $F_{2}$ of rank two are geometrical, most automorphisms of arbitrary free groups are not. For instance, the Tribonacci automorphism $1 \mapsto 12,2 \mapsto 13,3 \mapsto 1$ is not geometrical in the free group of rank three and more generally, no irreducible automorphism on a free group of odd rank comes from a homeomorphism of an orientable surface without boundary $[\mathbf{2 7}]$.

When considering automorphisms, the free group $F_{N}$ of rank $N$ plays the role of the monoid $\mathscr{Q}^{*}$ where $\mathscr{G}$ is the $N$ letter alphabet in the terminology of substitutions. The 
analog of infinite sequences as considered in the present monograph is given by the Gromov boundary of the free group. It is a Cantor set which compactifies $F_{N}$. Any automorphism of $F_{N}$ extends to the boundary of $F_{N}[66]$. The analog to a minimal symbolic dynamical system is then given by algebraic laminations. An attractive algebraic lamination of an automorphism is a set of geodesic lines in the free group which is closed (for the topology induced by the boundary topology), invariant under the action of the group and flip-invariant (i.e., orientation-invariant); therefore it is the analog of a substitutive dynamical system [70]. However, explicitly building such an attractive algebraic lamination is far from trivial; indeed, the constructions used for a substitution cannot be used since iterations of automorphisms of free groups produce cancelations so that infinite fixed words cannot be generated easily. An impressive achievement in this direction was obtained by Bestvina, Feighn and Handel [50]; the idea is to represent an automorphism of $F_{N}$ by a homotopy equivalence of a marked group $G$ with fundamental group $F_{N}$. In [52], the authors consider irreducible automorphisms with irreducible powers (iwip), which are the algebraic equivalent of pseudo-Anosov homeomorphisms of surfaces. They describe an algorithmic process to build a representative for the automorphism, called an improved relative train-track mapping, which takes care of cancellations so that one can build a reduced twosided recurrent infinite word on which the automorphism acts without cancellation and which is fixed by some power of the automorphism. From this, one deduces a symbolic dynamical system that is proved to be a representation of the attractive algebraic lamination [27].

Introducing the symbolic representation of an attractive lamination by means of a train track allows to geometrically represent the lamination by a central tile, as soon as the automorphism has a unit Pisot dilation coefficient [27]. However, the construction depends on the train-track used to represent the automorphism; additionally, automorphisms are considered in this context up to conjugacy by inner automorphisms. This is natural since a basic difference between the free monoid and the free group is that the free monoid has a canonical basis, which is not the case for the free group. Hence, although the attractive lamination is intrinsic, there exist several symbolic codings for it. It seems that deciding to choose a specific coding, hence a specific symbolic dynamical system, corresponds in particular to choosing a discrete time to move on the leaf of the formal lamination. The problem here is to understand which topological and metric properties of the central tile is invariant through conjugacy and the choice of the train-track representative. This will lead to the definition of a topological or metric invariant for free group automorphisms. The ultimate goal would be to propose a metric on the full lamination of an automorphism of the free group. In this direction, preliminary results in $[23, \mathbf{2 4}, \mathbf{6 7}, \mathbf{6 8}, \mathbf{6 9}]$ are of great interest: they illustrate why and how the central tile of the Tribonacci substitution is covered by an explicit Peano curve. 


\subsection{Effective constructions and generalizations}

To finish these perspectives, let us mention some concrete applications of substitutive dynamical systems. As already mentioned, central tiles are the fundament of an explicit Markov partition for the action of the incidence matrix of a substitution $\sigma$ on the torus. Let us consider this question in the reverse way. We consider a toral automorphism with a unit Pisot Perron-Frobenius eigenvalue and we look for a Markov partition. From the theory, we could actually choose as many partitions as we can build substitutions with the given matrix provided that its entries are nonnegative integers. In other words, we can build a substitution with the given matrix, then permute letters in the image of any letter, and we shall generate another Markov partition. At this stage, the crucial point is to propose criterions to choose a good Markov partition. By analogy with transformations of the interval, good Markov partitions shall be the ones for which two points located at a small distance have a quite long future in common. From this point of view, Markov partitions based on a central tile with a nontrivial fundamental group are not useful, since in this case many points of a piece of the partition are located at a small distance from another piece. Therefore for a given matrix with a unit Pisot Perron-Frobenius eigenvalue, the remaining question is: can we find a substitution corresponding to this matrix such that the pieces of the central tile are homeomorphic to a disk, or at least simply connected? As mentioned throughout the monograph, also for Pisot numbers that are not units, similar constructions can be performed. However, in order to get satisfactory results, we need to add $p$-adic factors to the space where we work in (see e.g. [154]). Recently, attempts were made in order to go beyond Pisot numbers. For instance, in [29] central tiles for a complex Pisot number are defined. For the case studied there, the expanding as well as the contracting space has dimension two. Details are much more complicated in this setting. Of course, it would be desirable to get further generalizations beyond the Pisot setting.

Similar considerations can be made in the field of discrete geometry. Let us consider a hyperplane $\mathbb{H}$ in $\mathbb{R}^{n}$. The discrete approximation of $\mathbb{H}$ is defined as the union of faces of unit cubes with integral coordinates that intersect $\mathbb{H}[\mathbf{2 6}]$. A specific case occurs when the hyperplane is orthogonal to the dominant eigenvector of a matrix with positive entries and with a Pisot unit of degree $n$ as dominant eigenvalue. In this case, any substitution with this matrix as incidence matrix is an irreducible unit Pisot substitution. Therefore, following the results stated in Chapter 3, the mapping $\mathbf{E}_{1}^{*}$ defined in Definition 3.4 stabilizes the discrete approximation of $\mathbb{H}$. From this we can derive a process to generate the discrete approximation of $\mathbb{H}$ : as soon as the substitution satisfies the geometric property $(\mathrm{F})$, applying $\left(\mathbf{E}_{1}^{*}\right)^{k}$ to the lower unit cube $\mathcal{U}$ produces increasing pieces of the approximation. However, in order to obtain suitable approximations, we need to check that the pieces are uniform [88]. This suggests that the choice of a good generator of a discrete surface (that is, a substitution with the suitable incidence matrix) should be guided by topological considerations on the central tile, such as simple connectivity and the fact that $\mathbf{0}$ is an inner point. To go 
further in that direction, a first strategy is to exhibit a relation between the topological properties of the finite approximations and their renormalized limit, which is the central tile. A second strategy is to generalize the topological characterizations described in this monograph in the context of the finite approximations. In other words, does there exist a graph that describes the connectivity of a finite approximation or its disklikeness?

A final motivation of this work is to control the production of any discrete plane. This refers to what is called Rauzy program in [43]: "find generalizations of the interaction between Sturmian words and irrational rotations which would naturally generate approximation algorithms". Looking at the Arnoux-Ito formalism [30], this means to start from an $n$-dimensional vector $\mathbf{v}$ in $\mathbb{R}^{n}$ and to decompose this vector with a continued fraction algorithm. Indeed, choose finitely many substitutions with incidence matrices $\mathbf{A}_{1}, \ldots, \mathbf{A}_{L}$ in an appropriate way. Then write $\mathbf{v}=\mathbf{M}_{1} \mathbf{M}_{2} \ldots \mathbf{M}_{k} \mathbf{v}_{k}$, say, where each of the matrices $\mathbf{M}_{j}$ is chosen from the finite set of matrices $\mathbf{A}_{1}, \ldots, \mathbf{A}_{L}$. Now associate the related substitution $\sigma_{j}$ to each matrix $\mathbf{M}_{j}$. Then, from $[\mathbf{2 8}]$, we know that the iterations of the dual substitutions $\mathbf{E}_{1}^{*}\left(\sigma_{1}\right) \mathbf{E}_{1}^{*}\left(\sigma_{2}\right) \ldots \mathbf{E}_{1}^{*}\left(\sigma_{k}\right)(\mathcal{U})^{(1)}$ generate increasing pieces of the discrete approximation for the hyperplane orthogonal to v. Many questions remain on this topic: can we cover the whole discrete plane with these iterations [99]? Can we renormalize the approximations and associate a central tile to the vector $\mathbf{v}$ ? Is such a central tile useful to produce simultaneous rational approximations of the coordinates of $\mathbf{v}$ ? Coming back to discrete geometry, two main questions appear. The first one is how to use this process in order to decide whether a discrete piece is indeed a part of a discrete approximation of a hyperplane. This question was tackled in $[\mathbf{4 4}, \mathbf{8 5}]$ by using the modified Jacobi-Perron algorithm. The second question is how to ensure that the pieces that are produced by iterations have a suitable shape? As explained in the substitutive case, we wish for approximations that contain no holes and are quite uniform. To answer this question, we need to build boundary graphs for the successive discrete approximations, taking into account that the substitution may change at each step and then take benefit of the structure of such a (infinite) graph.

(1) $\mathcal{U}$ is defined in (3.6). 


\section{BIBLIOGRAPHY}

[1] B. Adamczewski \& Y. Bugeaud - "On the complexity of algebraic numbers. I. Expansions in integer bases", Ann. of Math. 165 (2007), p. 547-565.

[2] B. Adamczewski, Y. Bugeaud \& L. Davison - "Continued fractions and transcendental numbers", Ann. Inst. Fourier (Grenoble) 56 (2006), p. 2093-2113.

[3] B. Adamczewski, C. Frougny, A. Siegel \& W. Steiner - "Rational numbers with purely periodic beta-expansion", J. London Math. Soc. 42 (2010), p. 538-552.

[4] R. L. Adler - "Symbolic dynamics and Markov partitions", Bull. Amer. Math. Soc. (N.S.) 35 (1998), p. 1-56.

[5] R. L. Adler \& B. Weiss - Similarity of automorphisms of the torus, Memoirs of the American Mathematical Society, No. 98, Amer. Math. Soc., 1970.

[6] S. Akiyama - "Pisot numbers and greedy algorithm", in Number theory (Eger, 1996), de Gruyter, 1998, p. 9-21.

[7] _ "Self affine tiling and Pisot numeration system", in Number theory and its applications (Kyoto, 1997), Dev. Math., vol. 2, Kluwer Acad. Publ., 1999, p. 7-17.

[8] _ "Cubic Pisot units with finite beta expansions", in Algebraic number theory and Diophantine analysis (Graz, 1998), de Gruyter, 2000, p. 11-26.

[9] _ "On the boundary of self affine tilings generated by Pisot numbers", $J$. Math. Soc. Japan 54 (2002), p. 283-308.

[10] _ "Pisot number system and its dual tiling", in Physics and Theoretical Computer Science (Cargese, 2006), IOS Press, 2007, p. 133-154.

[11] S. Akiyama, G. Barat, V. Berthé \& A. Siegel - "Boundary of central tiles associated with Pisot beta-numeration and purely periodic expansions", Monatsh. Math. 155 (2008), p. 377-419.

[12] S. Akiyama, T. Borbély, H. Brunotte, A. Pethö \& J. M. ThuswaldNER - "Generalized radix representations and dynamical systems. I", Acta Math. Hungar. 108 (2005), p. 207-238. 
[13] S. Akiyama, H. Brunotte, A. Pethő \& J. M. Thuswaldner - "Generalized radix representations and dynamical systems. II", Acta Arith. 121 (2006), p. 2161.

[14] _ "Generalized radix representations and dynamical systems. III", Osaka J. Math. 45 (2008), p. 347-374.

[15] "Generalized radix representations and dynamical systems. IV", Indag. Math. (N.S.) 19 (2008), p. 333-348.

[16] S. Akiyama, G. Dorfer, J. M. Thuswaldner \& R. Winkler - "On the fundamental group of the Sierpiński-gasket", Topology Appl. 156 (2009), p. 16551672 .

[17] S. Akiyama \& G. Nertila - "On the connectedness of self-affine tilings", Arch. Math. 82 (2004), p. 153-163.

[18] S. Akiyama, H. Rao \& W. Steiner - "A certain finiteness property of Pisot number systems", J. Number Theory 107 (2004), p. 135-160.

[19] S. Akiyama \& K. Scheicher - "Intersecting two-dimensional fractals with lines", Acta Sci. Math. (Szeged) 71 (2005), p. 555-580.

[20] C. Allauzen - "Une caractérisation simple des nombres de Sturm", J. Théor. Nombres Bordeaux 10 (1998), p. 237-241.

[21] J.-P. Allouche \& J. O. Shallit - Automatic sequences: Theory and applications, Cambridge Univ. Press, 2002.

[22] J. Anderson \& I. Putnam - "Topological invariants for substitution tilings and their associated $C^{*}$-algebras", Ergodic Theory Dynam. Systems 18 (1998), p. $509-537$.

[23] P. Arnoux - "Un exemple de semi-conjugaison entre un échange d'intervalles et une translation sur le tore", Bull. Soc. Math. France 116 (1988), p. 489-500.

[24] P. Arnoux, J. Bernat \& X. Bressaud - "Geometrical models for substitutions", Experiment. Math. (2010), to appear.

[25] P. Arnoux, V. Berthé, H. Ei \& S. Ito - "Tilings, quasicrystals, discrete planes, generalized substitutions, and multidimensional continued fractions", in Discrete models: combinatorics, computation, and geometry (Paris, 2001), Discrete Math. Theor. Comput. Sci. Proc., AA, Maison Inform. Math. Discrèt. (MIMD), Paris, 2001, p. 059-078.

[26] P. Arnoux, V. Berthé, T. Fernique \& D. Jamet - "Functional stepped surfaces, flips, and generalized substitutions", Theoret. Comput. Sci. 380 (2007), p. $251-265$. 
[27] P. Arnoux, V. Berthé, A. Hilion \& A. Siegel - "Fractal representation of the attractive lamination of an automorphism of the free group", Ann. Inst. Fourier (Grenoble) 56 (2006), p. 2161-2212.

[28] P. Arnoux, V. Berthé \& S. Ito - "Discrete planes, $\mathbb{Z}^{2}$-actions, Jacobi-Perron algorithm and substitutions", Ann. Inst. Fourier (Grenoble) 52 (2002), p. 305-349.

[29] P. Arnoux, M. Furukado, E. Harriss \& S. Ito - "Algebraic numbers, group automorphisms and substitution rules on the plane", Trans. Amer. Math. Soc. (2010), in press.

[30] P. Arnoux \& S. Ito - "Pisot substitutions and Rauzy fractals", Bull. Belg. Math. Soc. Simon Stevin 8 (2001), p. 181-207.

[31] V. BAKER, M. BARGE \& J. KWAPISZ - "Geometric realization and coincidence for reducible non-unimodular pisot tiling spaces with an application to beta-shifts", Ann. Inst. Fourier 56 (2006), p. 2213-2248.

[32] C. Bandt \& G. Gelbrich - "Classification of self-affine lattice tilings", $J$. London Math. Soc. 50 (1994), p. 581-593.

[33] G. Barat, V. Berthé, , P. Liardet \& J. M. Thuswaldner - "Dynamical directions in numeration", Ann. Inst. Fourier (Grenoble) 56 (2006), p. 1987-2092.

[34] M. Barge \& B. Diamond - "Coincidence for substitutions of Pisot type", Bull. Soc. Math. France 130 (2002), p. 619-626.

[35] M. Barge, B. Diamond \& R. Swanson - "The branch locus for onedimensional Pisot tiling spaces", Fund. Math. 204 (2009), p. 215-240.

[36] M. Barge \& J. KWAPISz - "Geometric theory of unimodular Pisot substitutions", Amer. J. Math. 128 (2006), p. 1219-1282.

[37] F. BAssino - "Beta-expansions for cubic Pisot numbers", in LATIN 2002: Theoretical informatics (Cancun), Lecture Notes in Comput. Sci., vol. 2286, Springer, 2002, p. 141-152.

[38] L. E. BAum \& M. M. SweET - "Continued fractions of algebraic power series in characteristic 2", Ann. of Math. 103 (1976), p. 593-610.

[39] M.-P. BÉAL \& D. PERRIN - "Symbolic dynamics and finite automata", in Handbook of Formal Languages (G. Rozenberg \& A. Salomaa, eds.), vol. 2, Springer, 1997, p. 463-503.

[40] J. Bernat - "Arithmetics in $\beta$-numeration", Discrete Math. Theor. Comput. Sci. 9 (2007), p. 85-106.

[41] _ "Computation of $L_{\oplus}$ for several cubic Pisot numbers", Discrete Math. Theor. Comput. Sci. 9 (2007), p. 175-193. 
[42] J. Berstel \& D. PERRIN - "The origins of combinatorics on words", European J. Combin. 28 (2007), p. 996-1022.

[43] V. Berthé, S. Ferenczi \& L. Q. Zamboni - "Interactions between dynamics, arithmetics and combinatorics: the good, the bad, and the ugly", in Algebraic and topological dynamics, Contemp. Math., vol. 385, Amer. Math. Soc., 2005, p. $333-364$.

[44] V. Berthé \& T. Fernique - "Brun expansions of stepped surfaces", Preprint (2010).

[45] V. Berthé \& A. Siegel - "Tilings associated with beta-numeration and substitutions", INTEGERS (Electronic Journal of Combinatorial Number Theory) 5 (2005).

[46] _ "Purely periodic $\beta$-expansions in the Pisot non-unit case", J. Number Theory 127 (2007), p. 153-172.

[47] V. Berthé, A. Siegel, W. Steiner, P. Surer \& J. M. Thuswaldner - "Fractal tiles associated with shift radix systems", Advances in Mathematics (2010), in press.

[48] V. Berthé, A. Siegel \& J. M. Thuswaldner - "Substitutions, Rauzy fractals, and tilings", in Combinatorics, Automata, and Number Theory (V. Berthé $\&$ M. Rigo, eds.), Encyclopedia of Mathematics and its Applications, Cambridge Univ. Press, to appear.

[49] A. Bertrand-Mathis - "Développement en base $\theta$; répartition modulo un de la suite $\left(x \theta^{n}\right)_{n \geq 0}$; langages codés et $\theta$-shift", Bull. Soc. Math. France 114 (1986), p. $271-323$.

[50] M. Bestvina, M. Feighn \& M. Handel - "Laminations, trees, and irreducible automorphisms of free groups", Geom. Funct. Anal. 7 (1997), p. 215-244.

[51] _ "Laminations, trees, and irreducible automorphisms of free groups", Geom. Funct. Anal. 7 (1997), p. 215-244.

[52] M. Bestvina \& M. HANDEL - "Train tracks and automorphisms of free groups", Ann. of Math. 135 (1992), p. 1-51.

[53] F. Blanchard - " $\beta$-expansions and symbolic dynamics", Theoret. Comput. Sci. 65 (1989), p. 131-141.

[54] E. BOMBIERI \& J. E. TAYLOR - "Which distributions of matter diffract? An initial investigation", J. Physique 47 (1986), p. C3-19-C3-28.

[55] R. BOWEN - Equilibrium states and the ergodic theory of Anosov diffeomorphisms, Lecture Notes in Math., vol. 470, Springer, 1975. 
[56] _ "Markov partitions are not smooth", Proc. Amer. Math. Soc. 71 (1978), p. $130-132$.

[57] C. Burdik, C. Frougny, J.-P. Gazeau \& R. Krejcar - "Beta-integers as natural counting systems for quasicrystals", J. of Physics A: Math. Gen. 31 (1998), p. 6449-6472.

[58] J. W. CANnON \& G. R. CONNER - "The combinatorial structure of the Hawaiian earring group", Topology Appl. 106 (2000), p. 225-271.

[59] V. CANTERINI - "Connectedness of geometric representation of substitutions of Pisot type", Bull. Belg. Math. Soc. Simon Stevin 10 (2003), p. 77-89.

[60] V. Canterini \& A. Siegel - "Geometric representation of substitutions of Pisot type", Trans. Amer. Math. Soc. 353 (2001), p. 5121-5144.

[61] J. Cassaigne, S. Ferenczi \& L. Q. Zamboni - "Imbalances in Arnoux-Rauzy sequences", Ann. Inst. Fourier (Grenoble) $\mathbf{5 0}$ (2000), p. 1265-1276.

[62] E. CAWLEY - "Smooth Markov partitions and toral automorphisms", Ergodic Theory Dynam. Systems 11 (1991), p. 633-651.

[63] N. Chenhova, P. Hubert \& A. Messaoudi - "Propriétés combinatoires, ergodiques et arithmétiques de la substitution de Tribonacci", J. Théor. Nombres Bordeaux 13 (2001), p. 371-394.

[64] A. Coвнам - "Uniform tag sequences", Math. Systems Theory 6 (1972), p. 164192.

[65] G. R. Conner \& J. W. Lamoreaux - "On the existence of universal covering spaces for metric spaces and subsets of the Euclidean plane", Fund. Math. 187 (2005), p. 95-110.

[66] D. COOPER - "Automorphisms of free groups have finitely generated fixed point sets", J. Algebra 111 (1987), p. 453-456.

[67] T. Coulbois, A. Hilion \& M. Lustig - "R-trees and laminations for free groups. I. Algebraic laminations", J. Lond. Math. Soc. 78 (2008), p. 723-736.

[68] , " $\mathbb{R}$-trees and laminations for free groups. II. The dual lamination of an R-tree", J. Lond. Math. Soc. 78 (2008), p. 737-754.

[69] , " $\mathbb{R}$-trees and laminations for free groups. III. Currents and dual $\mathbb{R}$-tree metrics", J. Lond. Math. Soc. 78 (2008), p. 755-766.

[70] , "R-trees, dual laminations and compact systems of partial isometries", Math. Proc. Cambridge Philos. Soc. 147 (2009), p. 345-368. 
[71] D. Crisp, W. Moran, A. Pollington \& P. Shiue - "Substitution invariant cutting sequences", J. Théor. Nombres Bordeaux 5 (1993), p. 123-137.

[72] F. M. DeKKING - "The spectrum of dynamical systems arising from substitutions of constant length", Z. Wahrscheinlichkeitstheorie und Verw. Gebiete 41 (1977/78), p. 221-239.

[73] O. Delgrange \& E. Rivals - "Star: an algorithm to search for tandem approximate repeats", Bioinformatics 20 (2004), p. 2812-20.

[74] J.-M. Dumont \& A. Thomas - "Systemes de numeration et fonctions fractales relatifs aux substitutions", Theoret. Comput. Sci. 65 (1989), p. 153-169.

[75] _ _ "Digital sum moments and substitutions", Acta Arith. 64 (1993), p. 205225.

[76] _ _Gaussian asymptotic properties of the sum-of-digits function", J. Number Theory 62 (1997), p. 19-38.

[77] F. DurAnd - "A generalization of Cobham's theorem", Theory Comput. Syst. 31 (1998), p. 169-185.

[78] F. Durand \& A. Messaoud - "Boundary of the rauzy fractal set in $\mathbb{R} \times \mathbb{C}$ generated by $p(x)=x^{4}-x^{3}-x^{2}-x-1$ ", Osaka J. of Math. (2010), in press.

[79] K. EdA \& K. KaWAmURA - "The fundamental groups of one-dimensional spaces", Topology Appl. 87 (1998), p. 163-172.

[80] H. Ei \& S. ITO - "Tilings from some non-irreducible Pisot substitutions", Discrete Math. Theor. Comput. Sci. 7 (2005), p. 81-122.

[81] H. Ei, S. ITO \& H. RAO - "Atomic surfaces, tilings and coincidences II. reducible case", Ann. Inst. Fourier 56 (2006), p. 2285-2313.

[82] M. Einsiedler \& K. SchmidT - "Markov partitions and homoclinic points of algebraic $\mathbf{Z}^{d}$-actions", Tr. Mat. Inst. Steklova 216 (1997), p. 265-284.

[83] K. FALCONER - Fractal geometry, Mathematical foundations and applications, John Wiley \& Sons Ltd., 1990.

[84] D.-J. Feng, M. Furukado, S. Ito \& J. Wu - "Pisot substitutions and the Hausdorff dimension of boundaries of atomic surfaces", Tsukuba J. Math. 30 (2006), p. 195-223.

[85] T. FERnique - "Generation and recognition of digital planes using multidimensional continued fractions", in Discrete geometry for computer imagery, Lecture Notes in Comput. Sci., vol. 4992, Springer, 2008, p. 33-44. 
[86] N. P. FogG - Substitutions in dynamics, arithmetics and combinatorics, Lecture Notes in Math., vol. 1794, Springer, 2002.

[87] C. Frougny \& B. Solomyak - "Finite beta-expansions", Ergodic Theory Dynam. Systems 12 (1992), p. 45-82.

[88] C. FuChs \& R. TiJdemAn - "Substitutions, abstract number systems and the space filling property", Ann. Inst. Fourier (Grenoble) 56 (2006), p. 2345-2389.

[89] B. Gaujal, A. HordiJK \& D. V. Der LAan - "On the optimal open-loop control policy for deterministic and exponential polling systems", Probability in Engineering and Informational Sciences 21 (2007), p. 157-187.

[90] J.-P. Gazeau \& J.-L. Verger-Gaugry - "Geometric study of the betaintegers for a Perron number and mathematical quasicrystals", J. Théor. Nombres Bordeaux 16 (2004), p. 125-149.

[91] M. HatA - "On the structure of self-similar sets", Japan J. Appl. Math. 2 (1985), p. $381-414$.

[92] G. A. Hedlund - "Remarks on the work of Axel Thue on sequences", Nordisk Mat. Tidskr. 15 (1967), p. 148-150.

[93] M. Hollander - "Linear numeration systems, finite beta expansions, and discrete spectrum of substitution dynamical systems", Ph.D. Thesis, University of Washington, 1996.

[94] P. Hubert \& A. Messaoudi - "Best simultaneous Diophantine approximations of Pisot numbers and Rauzy fractals", Acta Arith. 124 (2006), p. 1-15.

[95] S. ITO - "Simultaneous approximations and dynamical systems (on the simultaneous approximation of $\left(\alpha, \alpha^{2}\right)$ satisfying $\left.\alpha^{3}+k \alpha-1=0\right)$ ", Sürikaisekikenkyūsho Kōkyūroku 958 (1996), p. 59-61.

[96] S. Ito, J. Fujil, H. Higashinoand \& S.-I. Yasutomi - "On simultaneous approximation to $\left(\alpha, \alpha^{2}\right)$ with $\alpha^{3}+k \alpha-1=0$ ", J. Number Theory 99 (2003), p. $255-283$.

[97] S. Ito \& M. KimurA - "On Rauzy fractal", Japan J. Indust. Appl. Math. 8 (1991), p. 461-486.

[98] S. Iто \& M. Ohtsuki - "Modified Jacobi-Perron algorithm and generating Markov partitions for special hyperbolic toral automorphisms", Tokyo J. Math. 16 (1993), p. 441-472.

[99] _ "Parallelogram tilings and Jacobi-Perron algorithm", Tokyo J. Math. 17 (1994), p. 33-58. 
[100] S. Iто \& H. RAO - "Purely periodic $\beta$-expansion with Pisot base", Proc. Amer. Math. Soc. 133 (2005), p. 953-964.

[101] _ "Atomic surfaces, tilings and coincidences I. Irreducible case", Israel J. Math. 153 (2006), p. 129-155.

[102] C. Kalle \& W. Steiner - "Beta-expansions, natural extensions and multiple tilings", Trans. Amer. Math. Soc. (2010), in press.

[103] E. R. VAN KAMPEN - "On some characterizations of 2-dimensional manifolds", Duke Math. J. 1 (1935), p. 74-93.

[104] M. KEANE - "Interval exchange transformations", Math. Z. 141 (1975), p. 2531.

[105] J. Kellendonk \& I. Putnam - "Tilings, $C^{*}$-algebras, and $K$-theory", in $D i$ rections in mathematical quasicrystals (M. Baake et al., eds.), AMS CRM Monogr. Ser., vol. 13, 2000, p. 177-206.

[106] R. KENYON \& A. VERSHIK - "Arithmetic construction of sofic partitions of hyperbolic toral automorphisms", Ergodic Theory Dynam. Systems 18 (1998), p. $357-372$.

[107] K. Kuratowski - Topology. Vol. II, New edition, revised and augmented. Translated from the French by A. Kirkor, Academic Press, 1968.

[108] J. C. Lagarias \& Y. WANG - "Self affine tiles in $\mathbb{R}^{n}$ ", Adv. Math. 121 (1996), p. $21-49$.

[109] _ _ "Substitution Delone sets", Discrete Comput. Geom. 29 (2003), p. 175209.

[110] S. LE Borgne - "Un codage sofique des automorphismes hyperboliques du tore", in Séminaires de Probabilités de Rennes (1995), Publ. Inst. Rech. Math. Rennes, vol. 1995, Univ. Rennes I, 1995, p. 35.

[111] _ "Un codage sofique des automorphismes hyperboliques du tore", $C . R$. Acad. Sci. Paris Sér. I Math. 323 (1996), p. 1123-1128.

[112] _ _Un codage sofique des automorphismes hyperboliques du tore", Bol. Soc. Brasil. Mat. (N.S.) 30 (1999), p. 61-93.

[113] J.-Y. Lee, R. V. Moody \& B. Solomyak - "Pure point dynamical and diffraction spectra", Ann. Henri Poincaré 3 (2002), p. 1003-1018.

[114] D. Lind \& B. MARCUS - An introduction to symbolic dynamics and coding, Cambridge Univ. Press, 1995. 
[115] A. N. Livshits - "On the spectra of adic transformations of markov compacta", Uspekhi Mat. Nauk 42 (1987), p. 189-190.

[116] _ "Some examples of adic transformations and automorphisms of substitutions", Selecta Math. Soviet. 11 (1992), p. 83-104.

[117] B. Loridant \& J. M. Thuswaldner - "Interior components of a tile associated to a quadratic canonical number system", Topology Appl. 155 (2008), p. $667-695$.

[118] M. Lothaire - Applied combinatorics on words, Encyclopedia of Mathematics and its Applications, vol. 105, Cambridge Univ. Press, 2005.

[119] J. M. Luck, C. Godrèche, T. A. Janner \& Janssen - "The nature of the atomic surfaces of quasiperiodic self-similar structures", J. Phys. A 26 (1993), p. 1951-1999.

[120] J. LUO - "A note on a self-similar tiling generated by the minimal Pisot number", Fractals 10 (2002), p. 335-339.

[121] J. Luo, S. Akiyama \& J. M. Thuswaldner - "On the boundary connectedness of connected tiles", Math. Proc. Cambridge Philos. Soc. 137 (2004), p. 397410.

[122] J. LuO, H. RAO \& B. TAN - "Topological structure of self-similar sets", Fractals 10 (2002), p. 223-227.

[123] J. Luo \& J. M. Thuswaldner - "On the fundamental group of self-affine plane tiles", Ann. Inst. Fourier (Grenoble) 56 (2006), p. 2493-2524.

[124] J. Luo \& Z.-L. Zhou - "Disk-like tiles derived from complex bases", Acta Math. Sin. (Engl. Ser.) 20 (2004), p. 731-738.

[125] R. D. Mauldin \& S. C. Williams - "Hausdorff dimension in graph directed constructions", Trans. Amer. Math. Soc. 309 (1988), p. 811-829.

[126] A. Messaoud - "Propriétés arithmétiques et dynamiques du fractal de Rauzy", J. Théor. Nombres Bordeaux 10 (1998), p. 135-162.

[127] _ "Frontière du fractal de Rauzy et système de numération complexe", Acta Arith. 95 (2000), p. 195-224.

[128] _ _ "Propriétés arithmétiques et topologiques d'une classe d'ensembles fractales", Acta Arith. 121 (2006), p. 341-366.

[129] R. V. Moody - "Model sets: a survey", in From Quasicrystals to More Complex Systems (F. Axel \& J.-P. Gazeau, eds.), Les Editions de Physique, Springer, Berlin, 2000, p. $145-166$. 
[130] H. M. MorsE - "Recurrent geodesics on a surface of negative curvature", Trans. Amer. Math. Soc. 22 (1921), p. 84-100.

[131] B. Mossé - "Recognizability of substitutions and complexity of automatic sequences", Bull. Soc. Math. Fr. 124 (1996), p. 329-346.

[132] S.-M. NGai \& N. NGuYen - "The Heighway dragon revisited", Discrete Comput. Geom. 29 (2003), p. 603-623.

[133] S.-M. NGAi \& T.-M. TANG - "A technique in the topology of connected selfsimilar tiles", Fractals 12 (2004), p. 389-403.

[134] _ "Topology of connected self-similar tiles in the plane with disconnected interiors", Topology Appl. 150 (2005), p. 139-155.

[135] W. PARry - "On the $\beta$-expansion of real numbers", Acta Math. Acad. Sci. Hungar. 11 (1960), p. 401-416.

[136] B. Praggastis - "Numeration systems and Markov partitions from self-similar tilings", Trans. Amer. Math. Soc. 351 (1999), p. 3315-3349.

[137] N. PRIEBE-FRANCK - "A primer of substitution tilings of the Euclidean plane", Expo. Math. 26 (2008), p. 295-326.

[138] Y.-H. QU, H. RAO \& Y.-M. YANG - "Periods of $\beta$-expansions and linear recurrent sequences", Acta Arith. 120 (2005), p. 27-37.

[139] M. QUEFFÉLEC - Substitution dynamical systems-spectral analysis, Lecture Notes in Mathematics, 1294. Springer, 1987.

[140] C. RAdin - "Space tilings and substitutions", Geom. Dedicata 55 (1995), p. 257264.

[141] G. RAuZY - "Nombres algébriques et substitutions", Bull. Soc. Math. France 110 (1982), p. 147-178.

[142] J.-P. REVEILLÈS - "Géométrie discrète, calcul en nombres entiers et algorithmique", Thèse de Doctorat, Université Louis Pasteur, Strasbourg, 1991.

[143] M. Rigo \& W. Steiner - "Abstract $\beta$-expansions and ultimately periodic representations", J. Number Theory 17 (2005), p. 283-299.

[144] E. A. J. Robinson - "Symbolic dynamics and tilings of $\mathbb{R}^{d "}$, in Symbolic dynamics and its applications, Proc. Sympos. Appl. Math., Amer. Math. Soc. Providence, RI, vol. 60, 2004, p. 81-119.

[145] D. Roy - "Approximation to real numbers by cubic algebraic integers. II", Ann. of Math. 158 (2003), p. 1081-1087. 
[146] W. Rudin - "Some theorems on Fourier coefficients", Proc. Amer. Math. Soc. 10 (1959), p. 855-859.

[147] T. SADAhiro - "Multiple points of tilings associated with Pisot numeration systems", Theoret. Comput. Sci. 359 (2006), p. 133-147.

[148] Y. Sano, P. Arnoux \& S. Ito - "Higher dimensional extensions of substitutions and their dual maps", J. Anal. Math. 83 (2001), p. 183-206.

[149] K. Scheicher \& J. M. Thuswaldner - "Canonical number systems, counting automata and fractals", Math. Proc. Cambridge Philos. Soc. 133 (2002), p. 163182.

[150] K. SchmidT - "On periodic expansions of Pisot numbers and Salem numbers", Bull. London Math. Soc. 12 (1980), p. 269-278.

[151] _ _Algebraic coding of expansive group automorphisms and two-sided beta-shifts", Monatsh. Math. 129 (2000), p. 37-61.

[152] _ _Algebraic coding of expansive group automorphisms and two-sided beta-shifts", Monatsh. Math. 129 (2000), p. 37-61.

[153] M. Senechal - "What is... a quasicrystal?", Notices Amer. Math. Soc. 53 (2006), p. 886-887.

[154] A. SiEgEL - "Représentation des systèmes dynamiques substitutifs non unimodulaires", Ergodic Theory Dynam. Systems 23 (2003), p. 1247-1273.

[155] _ _Pure discrete spectrum dynamical system and periodic tiling associated with a substitution", Ann. Inst. Fourier (Grenoble) 54 (2004), p. 341-381.

[156] V. F. SIRVENT - "Geodesic laminations as geometric realizations of Pisot substitutions", Ergodic Theory Dynam. Systems 20 (2000), p. 1253-1266.

[157] V. F. Sirvent \& B. SolomyaK - "Pure discrete spectrum for one-dimensional substitution systems of Pisot type", Canad. Math. Bull. 45 (2002), p. 697-710.

[158] V. F. Sirvent \& Y. WANG - "Self-affine tiling via substitution dynamical systems and Rauzy fractals", Pacific J. Math. 206 (2002), p. 465-485.

[159] S. Smale - "Differentiable dynamical systems", Bull. Amer. Math. Soc. 73 (1967), p. 747-817.

[160] B. DE SMit - "The fundamental group of the Hawaiian earring is not free", Internat. J. Algebra Comput. 2 (1992), p. 33-37.

[161] B. SolomyAK - "Dynamics of self-similar tilings", Ergodic Theory Dynam. Systems 17 (1997), p. 695-738. 
[162] _ "Tilings and dynamics", in EMS Summer School on Combinatorics, Automata and Number Theory, 2006.

[163] W. Steiner - "Digital expansions and the distribution of related functions", 2000, http://www.liafa.jussieu.fr/ ${ }^{\sim}$ steiner/.

[164] A. Thue - "Über unendliche Zeichenreihen", Norske Vid. Selsk. Skr. Mat. Nat. Kl. 37 (1906), p. 1-22.

[165] _ _Über die gegenseitige Lage gleicher Teile gewisser Zeichenreihen", Norske Vid. Selsk. Skr. Mat. Nat. Kl. 43 (1912), p. 1-67.

[166] W. P. Thurston - "Groups, tilings and finite state automata", Lectures notes distributed in conjunction with the Colloquium Series, in AMS Colloquium lectures, 1989.

[167] J. M. Thuswaldner - "Unimodular Pisot substitutions and their associated tiles", J. Théor. Nombres Bordeaux 18 (2006), p. 487-536.

[168] W. A. VeECH - "Interval exchange transformations", J. Anal. Math. 33 (1978), p. $222-272$.

[169] R. F. Williams - "Classification of one dimensional attractors", in Global Analysis (Proc. Sympos. Pure Math., Vol. XIV, Berkeley, Calif., 1968), Amer. Math. Soc., 1970, p. 341-361.

[170] S.-I. Yasutomi - "On Sturmian sequences which are invariant under some substitutions", in Number theory and its applications (Kyoto, 1997), Dev. Math., vol. 2, Kluwer Acad. Publ., 1999, p. 347-373. 